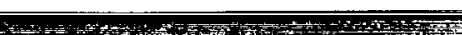

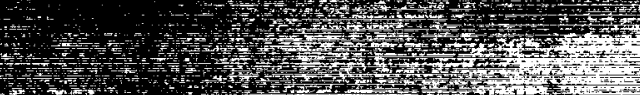

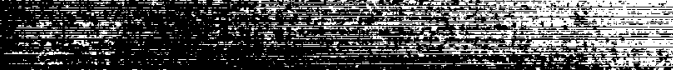

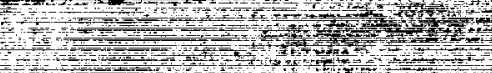

that

H.

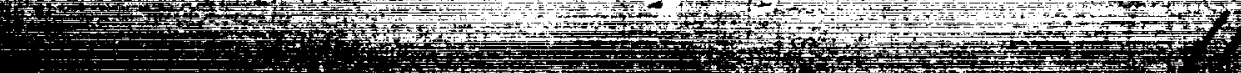

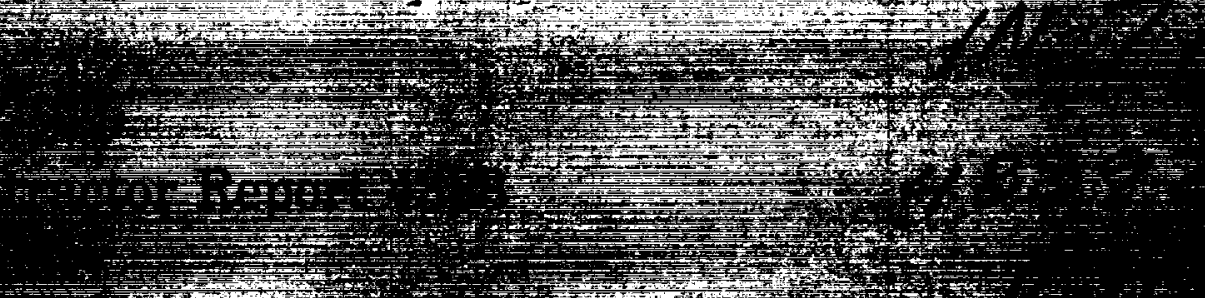

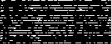

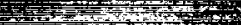

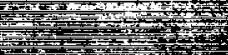

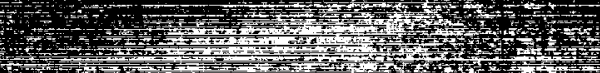

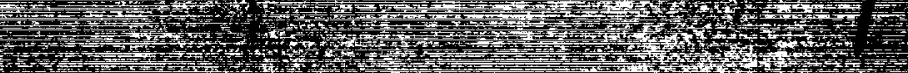

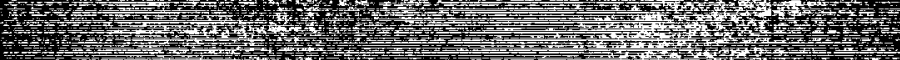

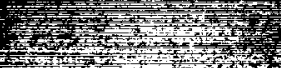

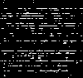

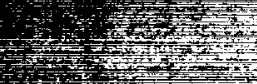

13tint

and

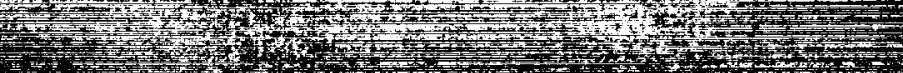

ant?

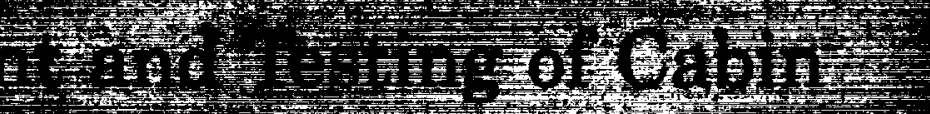

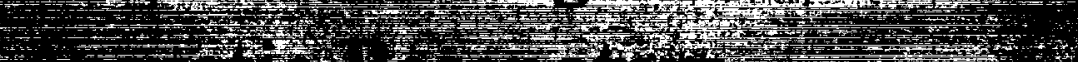

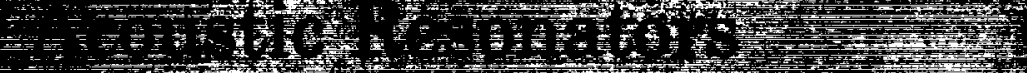

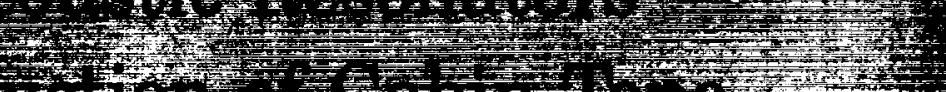

W

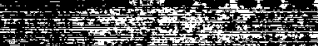

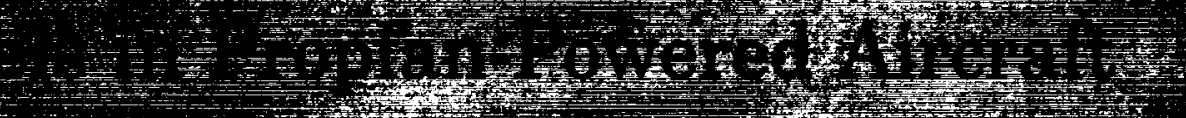

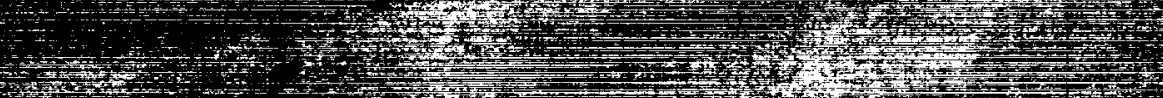

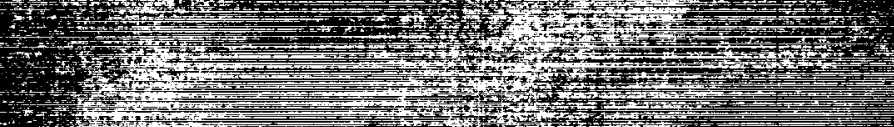

4 (1)

174.

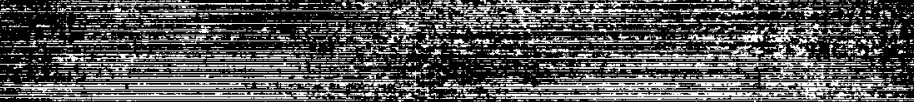

m.1.

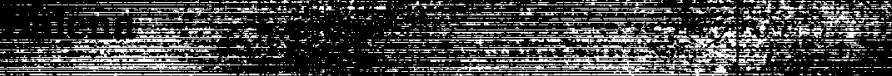

1.

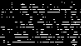

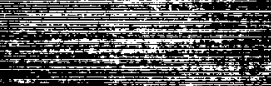

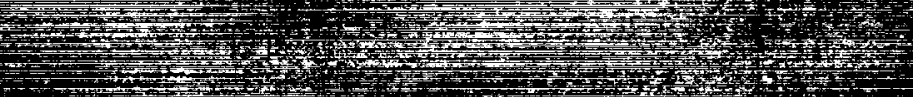

1.

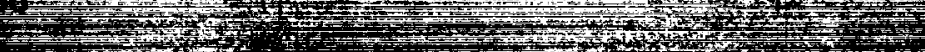

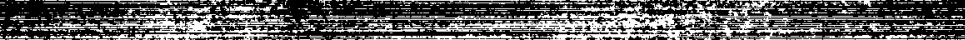

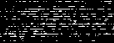

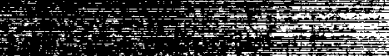

H.

H.

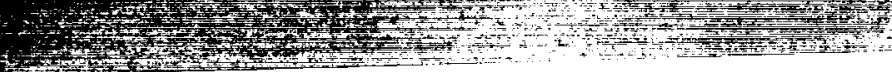

$\frac{1}{4}$

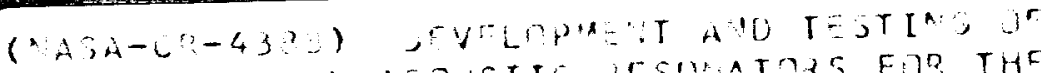

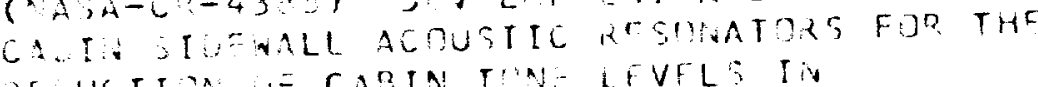

REACTION j= CAMIA TOME LFVFLS IN

$93,-$

I IKC ART

inal Report,

rict.

inc.

$92-13757$

$+1$

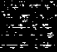

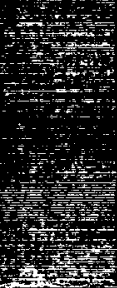

$41 / 71$

Unclis

(Lorkhst's A aro

0043392 


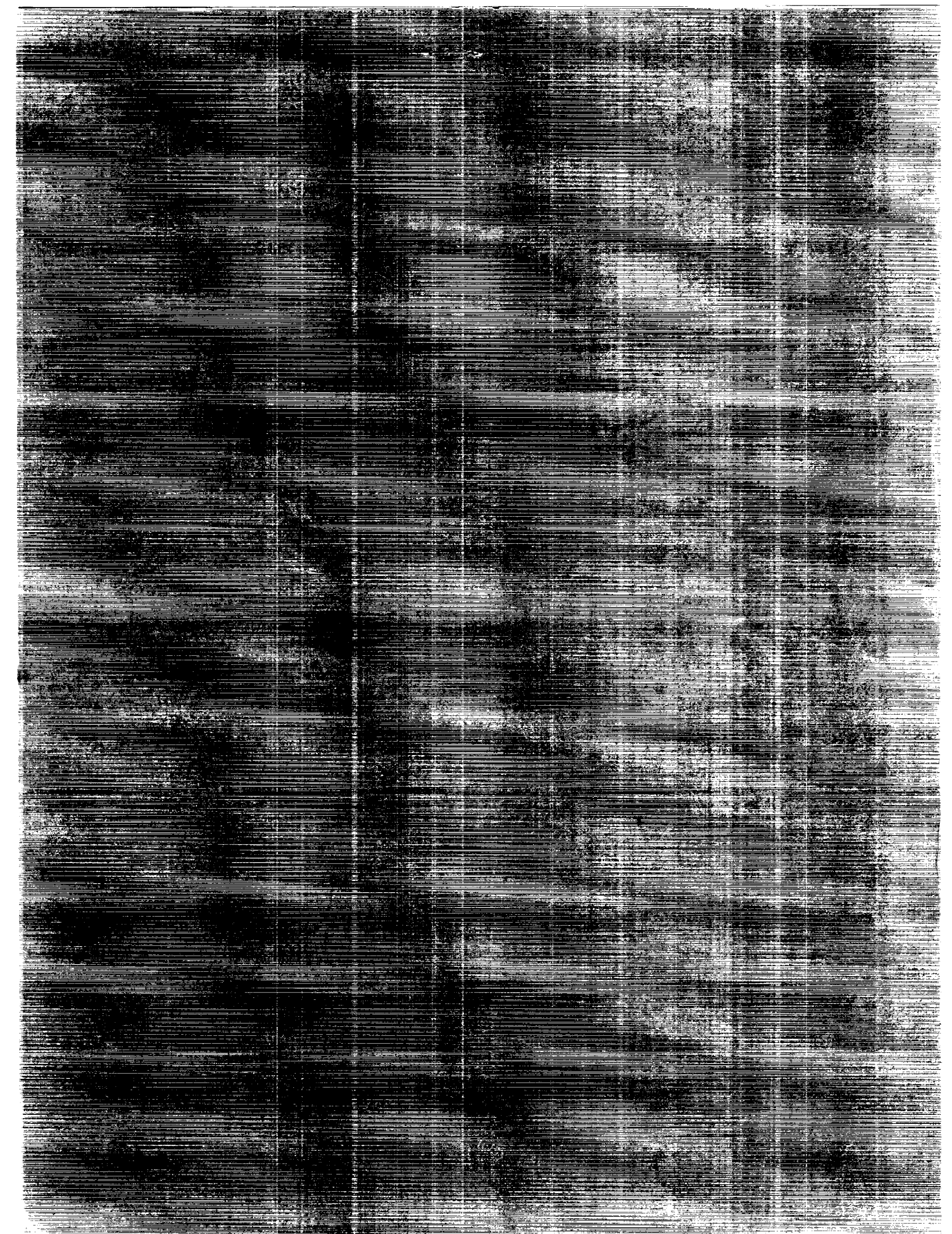


NASA Contractor Report 4388

\section{Development and Testing of Cabin Sidewall Acoustic Resonators for the Reduction of Cabin Tone Levels in Propfan-Powered Aircraft}

H. L. Kuntz, R. J. Gatineau,

R. A. Prydz, and F. J. Balena

Lockheed Aeronautical Systems Company

A Division of Lockheed Corporation

Burbank, California

Prepared for

Langley Research Center

under Contract NAS1-18036

\section{N/SA}

National Aeronautics and

Space Administration

Office of Management

Scientific and Technical

Information Program 


\section{FOREWORD}

The work described herein was performed by Lockheed Aeronautical Systems Company (LASC) -Burbank during the period of October 1986 through December 1990. The program emphasis was on reducing high sound pressure level propfan tones in the NASA-Lewis/Lockheed-Georgia Propfan Test Assessment (PTA) aircraft cabin. Analytical and experimental research was performed on the noise reduction (NR) of flat double-panel wall assemblies containing acoustic resonators. Subsequent laboratory and flight tests were performed with a cabin trim and resonator-support structure built for the PTA test aircraft.

A number of personnel worked on this project in California and in Georgia. L.S. Wirt and D.L. Morrow developed and refined the flight test resonators. D.L. Morrow, J.L. Hayward, Carl J. Looper, H.L. Kuntz, E.P. Feltz, and J.R. Trott organized and performed laboratory research and development tests involving: a laboratory fuselage mockup section, flat panels, active sound cancellation, and individual resonators. R.J. Gatineau designed the acoustic enclosure (cabin trim and resonator-support structure) and assisted R.A. Prydz in overseeing the acoustic enclosure flight tests, which were performed by Georgia personnel. The LASC-Georgia PTA Flight Test Department, headed by D.T. Poland, provided valuable design specifications for the enclosure. A LASC-Georgia engineering team, directed by R.W. Nazarowski, reviewed the design of the enclosure and provided CADAM drawings for the final construction phase. J.R. Withers, Chris J. Looper, M. Adames, D.F. DeFrancis, and others worked on the Acoustics Laboratory TL facility and laboratory fuselage section construction and modifications. W.G. Thomas, D.M. Dean, E.D. Rollo, and others fabricated the acoustic enclosure at Rye Canyon. D.L. Morrow and H.L. Kuntz developed double-tuned resonators for testing. As part of this contract, L.D. Pope (L.D. Pope \& Associates) modified the Propeller Aircraft Interior Noise (PAIN) computer program, which is used to predict aircraft interior sound pressure levels. This modification implemented a theoretical addition of resonators within the walls formed by the cabin trim and the fuselage shell.

R.A. Prydz was Program Manager/Principal Investigator from October 1986 through August 1988; F.J. Balena was Program Manager/Principal Investigator from September 1988 through August 1989; and H.L. Kuntz was Program Manager/ Principal Investigator from September 1989 through project completion.

D.G. Stephens was NASA Technical Contract Monitor from 0ctober 1986 through December 1987. K.P. Shepherd was NASA Technical Contract Monitor from January 1988 through project completion. The work described in this report was funded by NASA-Langley Research Center contract NAS1-18036. The flight tests were performed at LASC-Georgia in conjunction with tests performed under the NASALewis Research Center Propfan Test Assessment (PTA) Contract NAS3-24339 from October 1985 through July 1989.

This report was originally printed as LASC Lockheed Engineering Report LG90ER0119 (October 1990). 


\section{SUHMARY}

From January 1987 through December 1990 design, testing, and analyses were performed to evaluate the use of Helmholtz resonators in aircraft cabin sidewalls. The Helmholtz resonators are used to increase the transmission loss (TL) of the sidewalls at propfan blade passage frequencies. The primary development work consisted of designing and testing a Helmholtz resonator which had a well-defined response and could fit into the sidewall of a trimmed Gulfstream II aircraft. The development and design work consisted of integrating Helmholtz resonators within a special aircraft cabin trimsimulating enclosure. The enclosure was built and tested in the Rye Canyon, CA, Acoustics Laboratory, and was flight tested in the Propfan Test Assessment aircraft in Georgia. The analysis work consisted of parameter analyses on the performance of single resonators, of resonators in double-panel wall assemblies, and of resonators mounted inside the fuselage cabin trim. This final report summarizes the work performed during this project.

The results of this investigation show that the resonators increase the TL of double-panel structures at and near the design resonance frequencies of the resonators. Resonators may be tuned to the blade passage frequencies of the propfan or to any other specific, narrow frequency bandwidth where the TL needs to be increased. Flat panel tests showed that the resonators greatly improved the sidewall TL for a narrow frequency bandwidth. Flight testing and laboratory fuselage testing showed TL increases over a fairly broad frequency range and a significant increase in the wall peak TL values at the resonance frequency of the resonators. The reasons for the $\mathrm{TL}$ differences between flat double-panel walls and flight test curved wall configurations were explored and are explained herein.

In addition to investigating the use of single-frequency Helmholtz resonators within cabin sidewalls, other methods of increasing double-panel wall TL were developed and explored. Three different types of double-tuned resonators were developed and two types were tested in flat double-panel walls. In addition, the effectiveness of active sound cancellation was tested within flat doublepanel wall assemblies. The results of these latter tests show promise, but the results are preliminary and further development testing is suggested. 


\section{TABLE OF CONTENTS}

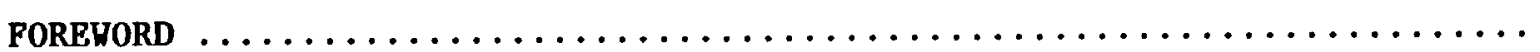

SUMMARY

LIST OP FIGURES

NOTATION

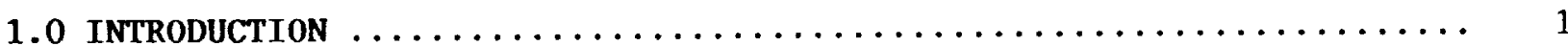

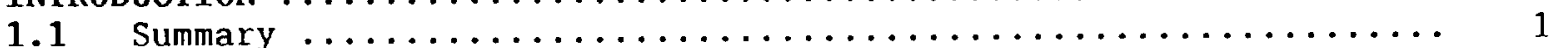

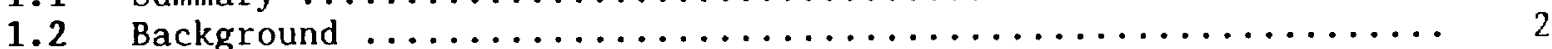

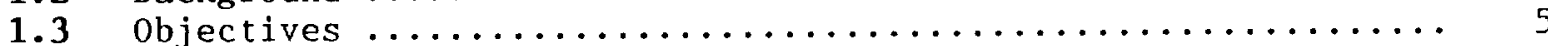

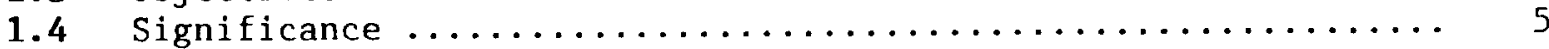

2.0 RESONATOR DESIGN $\ldots \ldots \ldots \ldots \ldots \ldots \ldots \ldots \ldots \ldots \ldots \ldots \ldots \ldots \ldots \ldots$

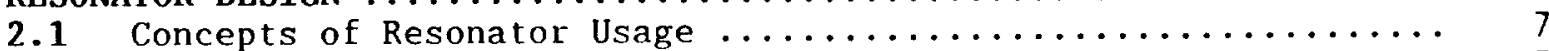

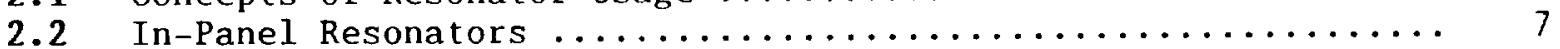

2.3 Illustration of the Double-Panel Assembly Resonance ........ 10

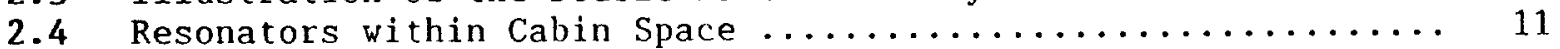

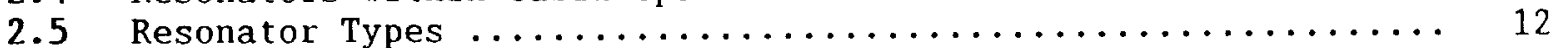

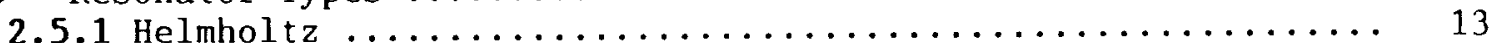

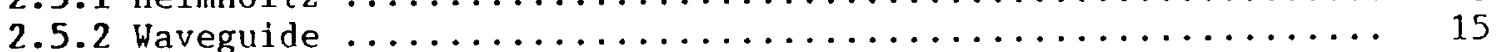

2.5.3 Half and Quarter Wavelength Resonators ............. 16

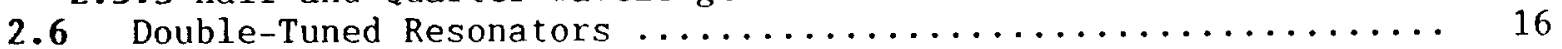

2.6.1 Double Helmholtz Resonator ..................... 16

2.6.2 Double-Tuned Waveguide Resonator $\ldots \ldots \ldots \ldots \ldots \ldots \ldots \ldots \ldots \ldots \ldots \ldots$

2.6.3 Cascaded Helmholtz Resonator .................... 17

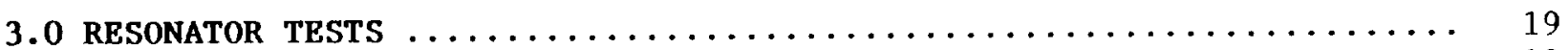

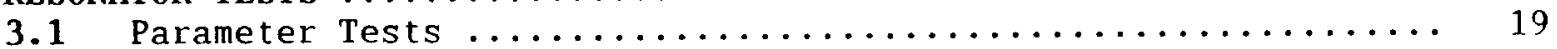

3.2 Free-Field Measurement of Nonlinear Behavior $\ldots \ldots \ldots \ldots \ldots \ldots . \ldots 21$

3.3 Impedance Tube Measurement of Nonlinear Behavior .......... 21

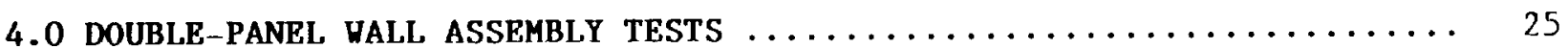

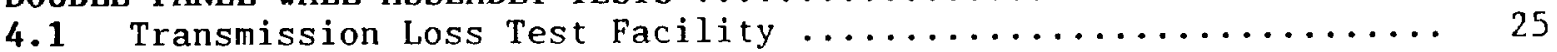

4.2 NR Tests of Double-Panel Wall Assemblies with Internal

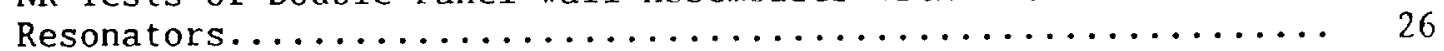

4.2.1 Single-Tuned Helmholtz Resonators ................ 26

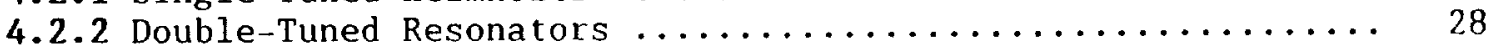

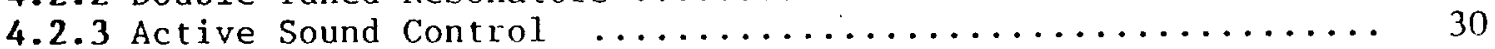

5.0 FLIGHT TEST ACOUSTIC ENCLOSURE DESIGN $\ldots \ldots \ldots \ldots \ldots \ldots \ldots \ldots \ldots \ldots$ 


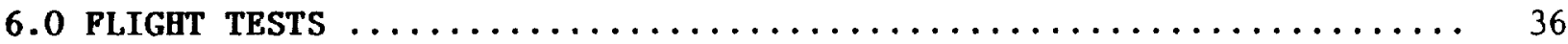

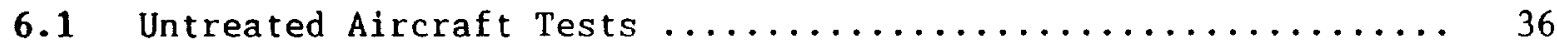

6.1.1 Ground Tests $\ldots \ldots \ldots \ldots \ldots \ldots \ldots \ldots \ldots \ldots \ldots \ldots \ldots \ldots \ldots,{ }_{36}$

6.1.2 Flight Tests $\ldots \ldots \ldots \ldots \ldots \ldots \ldots \ldots \ldots \ldots \ldots \ldots \ldots \ldots \ldots \ldots \ldots,{ }_{37}$

6.2 Acoustic Enclosure Flight Tests $\ldots \ldots \ldots \ldots \ldots \ldots \ldots \ldots \ldots . . \ldots, 39$

6.2.1 Interior Acoustic Treatment $\ldots \ldots \ldots \ldots \ldots \ldots \ldots \ldots \ldots \ldots, 39$

6.2.2 Resonator Effects $\ldots \ldots \ldots \ldots \ldots \ldots \ldots \ldots \ldots \ldots \ldots \ldots \ldots, 41$

6.2.3 Altitude Effects on Cabin Noise $\ldots \ldots \ldots \ldots \ldots \ldots \ldots \ldots, 42$

6.2.4 Vibration Isolation Effects $\ldots \ldots \ldots \ldots \ldots \ldots \ldots \ldots \ldots \ldots, 43$

6.2.5 Enclosure Absorption Effects $\ldots \ldots \ldots \ldots \ldots \ldots \ldots \ldots \ldots, 43$

7.0 LABORATORY PUSEILAGE/ENCLOSURE TESTS $\ldots \ldots \ldots \ldots \ldots \ldots \ldots \ldots \ldots \ldots, 45$

7.1 Fuselage Excitation Signals $\ldots \ldots \ldots \ldots \ldots \ldots \ldots \ldots \ldots \ldots \ldots \ldots \ldots \ldots \ldots \ldots, 45$

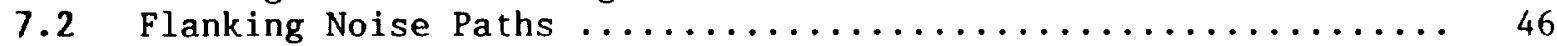

7.3 Individual Resonator Response $\ldots \ldots \ldots \ldots \ldots \ldots \ldots \ldots \ldots \ldots, 47$

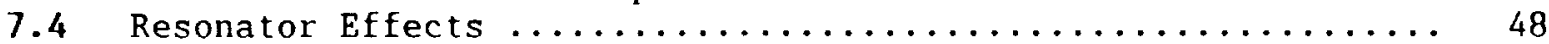

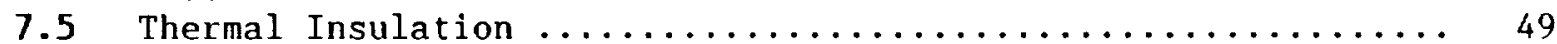

7.6 Sidewall/Under-Floor Measurements $\ldots \ldots \ldots \ldots \ldots \ldots \ldots \ldots .60$

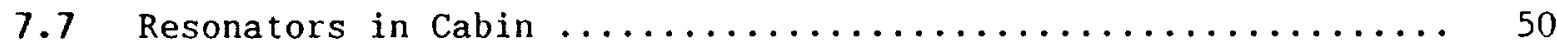

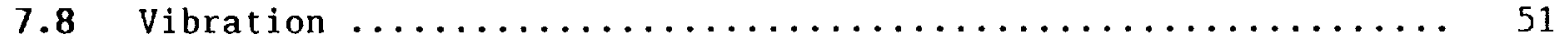

7.9 Recommended Additional Fuselage/Enclosure Testing........... 52

8.0 Conclusions $\ldots \ldots \ldots \ldots \ldots \ldots \ldots \ldots \ldots \ldots \ldots \ldots \ldots \ldots \ldots \ldots, 53$

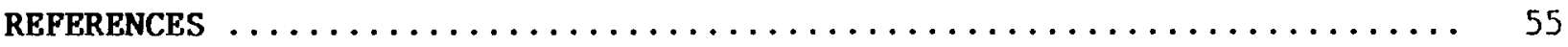

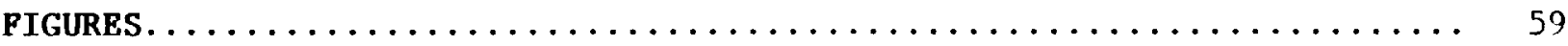




\section{LIST OF FIGURES}

\section{REPORT SECTION}

Figure Numbers:

Page

INTRODUCTION :

1: Project Timetable............................... 59

2: Sketch of PTA Aircraft with Wing-Mounted Tractor Propfan......... 60

3: Flight Test Hemispherical Resonator Configuration.............. 61

4: Photograph of Trim Panel Installation in Enclosure with Resonators

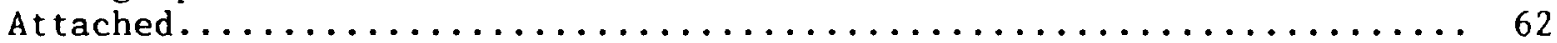

5: Photograph of Acoustics Laboratory with the Fuselage Section and EPT-

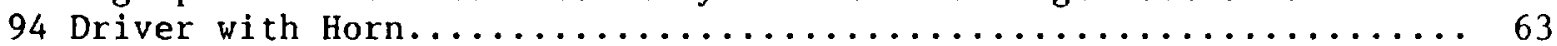

\section{RESONATOR DESIGN:}

6: Flat Double-Panel Wall Assembly with Resonators.............. 64

7: Predicted NRs of Wall Assemblies with Single-Panel, Double-Panel, and Double-Panel with Helmholtz Resonators..................... 65

8: Predicted NRs of a Double-Panel Wall Assembly with Helmholtz Resonators Tuned to One Frequency $(234 \mathrm{~Hz})$ and Then Tuned to Two

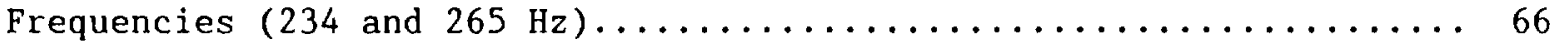

9: Theoretical NR of Double-Panel Wall Assembly with Helmholtz Resonators Predicted by Two Different Methods........................ 67

10: SPL Spectra in a Volume with and without Helmholtz Resonators. ..... 68

11: Photographs of Three Types of Double-Tuned Resonators........... 69

12: Four Different Helmholtz Resonator Configurations............ 70

13: Drawing of a Waveguide Resonator..................... 71

14: Drawing of a Double Helmholtz Resonator................. 72

15: Drawing of a Double-Tuned Waveguide Resonator.............. 73

16: Drawing of a Cascaded Helmholtz Resonator................ 74

\section{RESONATOR TESTS :}

17: Test Setup - Evaluation of Individual Resonators............. 75

18: Photograph of the Impedance Tube..................... 76 
19: Effect of Small Leaks on the Response of a Hemispherical Helmholtz Resonator.................................. 77

20: Effect of Various End Closures on the Response of an Hemispherical Helmholtz Resonator............................ 78

21: Amplification Responses of Three Double-Tuned Resonators.......... 79

22: Maximum Amplification versus Driving SPL for Three Double-Tuned Resonators.................................. 80

23: Quality Factor versus Driving SPL for Three Double-Tuned Resonators. 81

24: Reactive Part of the Impedance at Resonance versus Driving SPL for Three Double-Tuned Resonators.......................... 82

25: Resistive Part of the Impedance at Resonance versus Driving SPL for Three Double-Tuned Resonators......................... 83

26: Resistive Part of the Impedance versus Frequency for the Cascade

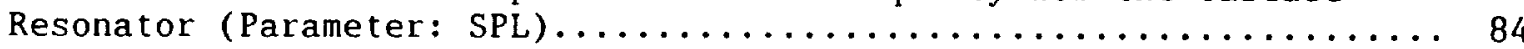

27: Absorption Coefficient at the Resonance Frequencies versus Driving SPL for Three Double-Tuned Resonators................... 85

28: Absorption Coefficient versus Frequency for the Cascade Resonator

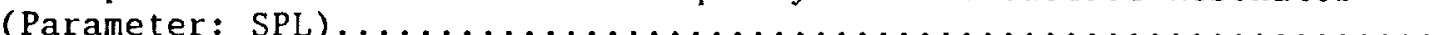

PANEL DESIGN :

29: Sound Transmission Facility for the Evaluation of Noise Reduction of

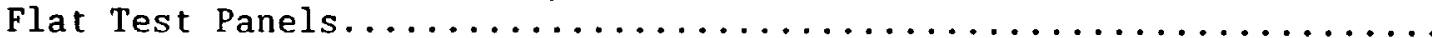

30: Sketch of the Sound Transmission Test Setup............... 88

31: Transmission Loss Test Fixture $(1.118$ by $1.118 \mathrm{~m}) \ldots \ldots \ldots \ldots \ldots$

32: Sketch of Microphone and Accelerometer Locations on the Test Panel... 90

33: The Four Basic Double-Panel Wall Assembly Test Configurations....... 91

PANEL TESTS:

34: Effect of Resonators on the NR of a Double-Panel Wall Assembly with Aluminum Panels $(234 \mathrm{~Hz}$ Tuning)............................. 92

35: Effect of Glass Fiber Batts on the NR of Double-Panel Wall Assembly with Aluminum Panels and Resonators $(207.5 \mathrm{~Hz}$ Tuning).......... 93

36: Effect of Air Space on NR of a Double-Panel Wall Assembly with Aluminum Panels and Resonators $(207.5 \mathrm{~Hz}$ Tuning)............. 94 
37: Effect of Resonator Count on NR of a Double-Panel Wall Assembly with

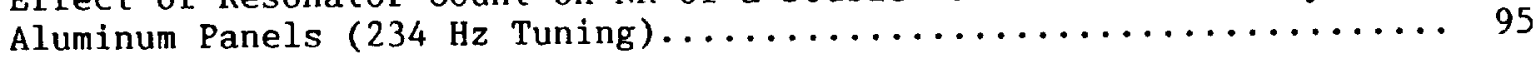

38: NR of a Single and Double-Tuned Resonator Array in a Double-Panel Wall Assembly with Aluminum Panels (234 and $265 \mathrm{~Hz}$ Tuning)........996

39: Effect of Resonators on NR of a Limp Double-Panel Wall Assembly (234

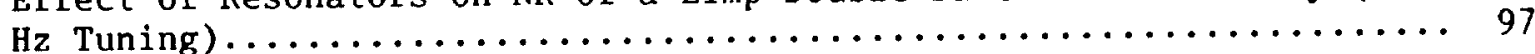

40: NR Test Results Measured in a Limp Double-Panel Wall Assembly with and without Waveguide Resonators at Two Frequencies.............. 98

41: NR Test Results Measured in a Limp Double-Panel Wall Assembly with and without Cascade Resonators at Two Frequencies............... 99

42: Photograph of a Single Active Control Loudspeaker and Single Reference Microphone in TL Facilty without Glass Fiber Batts and Receive Side Panel............................... 100

43: Photograph of Partition, Four Active Control Loudspeakers, and Four Reference Microphones in TL Facilty with Glass Fiber Batts and wi thout Receive Side Panel............................ 101

44: SPL Measured at a Microphone At $1 \mathrm{~m}$ from Receive Side Panel without and with the Single Active Control Loudspeaker operating......... 102

45: Measured NR Increase with a Single Active Noise Control Loudspeaker without and with $0.1 \mathrm{~m}$ Thick Glass Fiber Batts Between Panels...... 103

46: Measured NR Increase with a Single Active Control Loudspeaker without and with $0.1 \mathrm{~m}$ Thick Glass Fiber Batts Between Panels............ 104

47: Measured NR Increase with Four Active Control Loudspeakers without and with $0.1 \mathrm{~m}$ Thick Glass Fiber Batts Between Panels............ 105

\section{ENCLOSURE DESIGN:}

48: Photograph of Acoustic Enclosure Mounted on Plywood Assembly and Shipping Base................................ 106

49: PTA Enclosure Frame Section Properties.................. 107

50: Closeup Photograph of Enclosure Framework During Construction....... 108

51: Closeup Photograph of Junction between Circular Frames and Floor

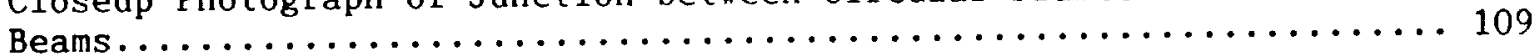

52: Closeup Photograph of Kick Plate Installation and Side View of a

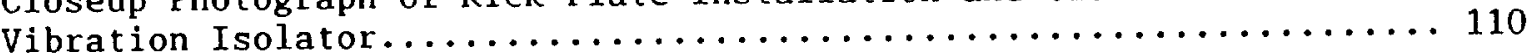




\section{FLIGHT TESTS:}

53: Cabin Microphone Designations and Locations Superimposed on Cabin

Cross Sections.................................... 111

54: Dependence of Tonal SPLs at a Cabin Microphone in the Prop Plane versus the Relative Input Force Level for the Three Force Locations.. 112

55: Fuselage SPLs Measured at the First Three Harmonics and at an

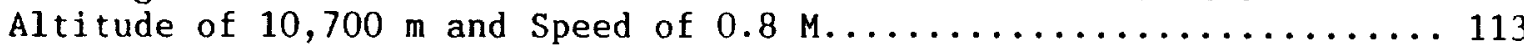

56: Fuselage SPL Spectra Measured $63.5 \mathrm{~cm}$ Behind the Prop Plane with and without the Propfan at an Altitude of $10,700 \mathrm{~m}$ and Speed of $0.8 \mathrm{M} \ldots 114$

57: Cabin SPL Spectra Measured in the Prop Plane with and without the Propfan at an Altitude of $10,700 \mathrm{~m}$ and Speed of $0.8 \mathrm{M} \ldots \ldots \ldots \ldots 114$

58: Fuselage and Cabin 〈SPL〉s versus BPF and Four Average Shaft Powers at

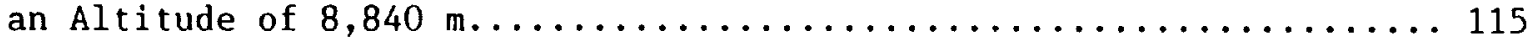

59: 〈NR $>$ s versus BPF at Four Altitudes (Symbols as in Fig. 58) ...... 116

60: Cabin 〈SPL〉S for the First Three BPF Harmonics Measured at Two Altitudes and Predicted from the Ground/Flight Wing Acceleration and Fuselage SPL Analyses............................ 117

61: Cabin Interior Arrangement with Acoustic Treatment............ 118

62: Fuselage Surface Microphone Locations................. 118

63: Interior Microphone and Accelerometer Locations............. 119

64: Resonator and Enclosure Effects on the Fundamental Tone 〈NR〉 at an

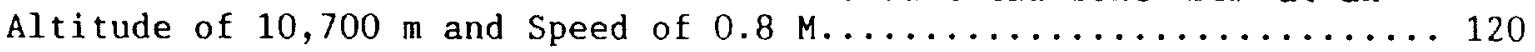

65: Chamber End Flanking Effect on the Fundamental Tone 〈NR〉 at an

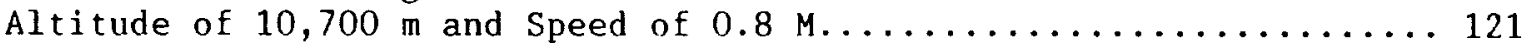

66: Normalized A-Weighted Cabin Sound Levels for Three Cases (7 Harmonic Average) at an Altitude of $10,700 \mathrm{~m}$ and speed of $0.8 \mathrm{M} \ldots \ldots \ldots \ldots 122$

67: Altitude Effect of Cabin Propfan Fundamental Tone 〈NR〉 - Resonators

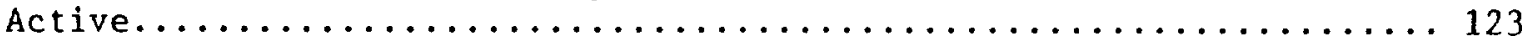

68: Altitude Effect of Cabin Propfan Fundamental Tone 〈NR〉 - Resonators

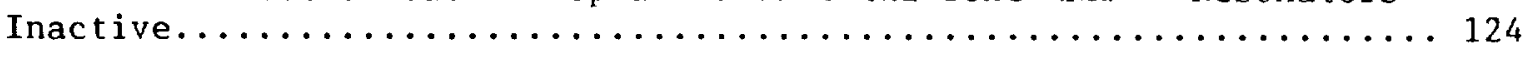

69: Vibration Isolation Effect on Cabin Fundamental Tone 〈NR〉 -

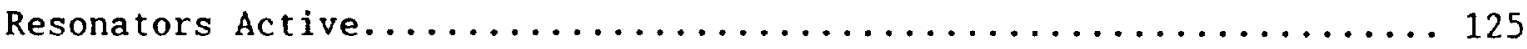


70: Vibration Isolation Effect on Cabin Second Harmonic Tone 〈NR〉 -

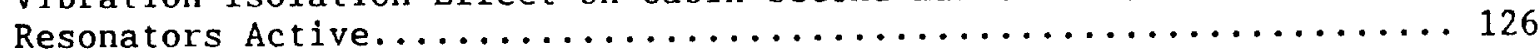

71: Vibration Isolation Effect on Cabin Third Harmonic Tone 〈NR〉 -

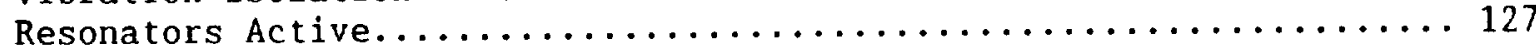

72: Cabin Absorption Effect on Cabin Propfan Fundamental Tone 〈NR〉 -

Resonators Removed............................... 128

73: Cabin Absorption Effect on Cabin Propfan Second Harmonic Tone 〈NR〉 Resonators Removed.................................. 129

74: Cabin Absorption Effect on Cabin Propfan Third Harmonic Tone 〈NR〉 -

Resonators Removed.............................. 130

\section{LABORATORY TESTS:}

75: Flight and Laboratory Fuselage 〈SPL〉s at Selected Propeller Fundamental Blade Passage Frequencies........................ 131

76: Typical Laboratory Input Tone Spectrum at the External Microphone

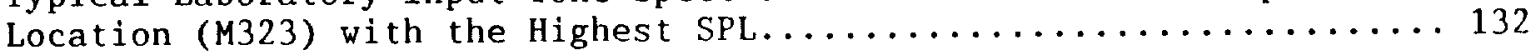

77: Typical Laboratory Random Noise Input Spectrum at the External Microphone Location (M323) with the Highest SPL.............. 133

78: Enclosure $\langle\mathrm{NR}\rangle \mathrm{s}$ with and without Barriers Seals and Enclosure End Wall Resonators - Fundamental BPF Tones.................... 134

79: Enclosure 〈NR>s with and without Barrier Seals and Enclosure End Wall

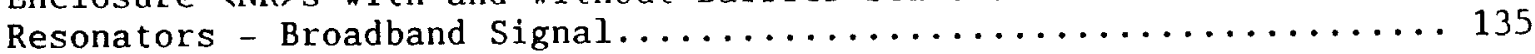

80: Probe Microphone Measurements of Resonator and Sidewall SPL Spectra with the Resonators Active - Broadband Noise Excitation.......... 136

81: Amplification Responses of a Resonator in the Fuselage Sidewall at Two Different Locations - Broadband Noise Excitation............ 137

82: Enclosure $\langle\mathrm{NR}\rangle \mathrm{s}$ with and without Sidewall Thermal Insulation in Place

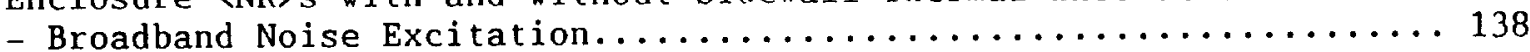

83: Enclosure $\langle\mathrm{NR}\rangle \mathrm{s}$ with and without Sidewall Thermal Insulation in Place

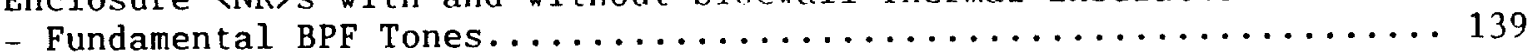

84: Effect of Sidewall Thermal Blanket Installation on Resonator Activity

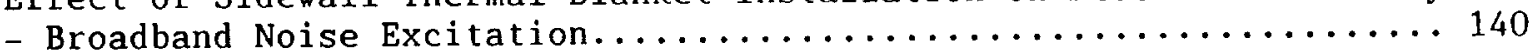

85: Fuselage/Enclosure 〈NR $>$ obtained in the Laboratory with Different Trim Panel Configurations - Broadband Noise Excitation........... 141 
86: Fuselage/Enclosure 〈NR〉 Obtained in the Laboratory with Different Trim Panel Configurations - Fundamental BPF Tones.............. 142

87: Central Sidewall Cavity Microphone Tonal NR Comparisons of Flight and Laboratory Data - Fundamental BPF Tones.................... 143

88: Normalized 〈SPL〉 Response Comparison of Sidewall and Under-Floor Spectra - Broadband Noise Excitation..................... 144

89: Fuselage/Enclosure 〈NR〉 Obtained in Laboratory with Active Resonators within the Sidewall versus Active Resonators within the Enclosure -

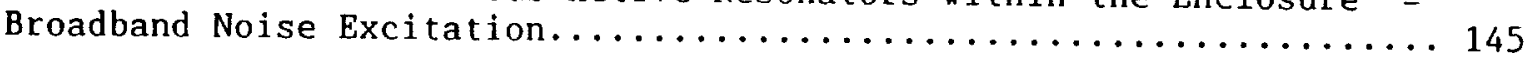




\section{LIST OP TABLES}

I: Table of First Approximations of Resonator Nonlinear Acoustic Flow

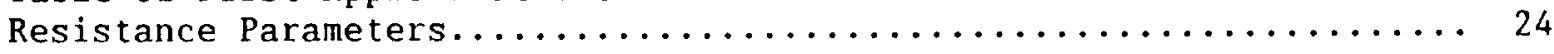




\section{NOTATION}

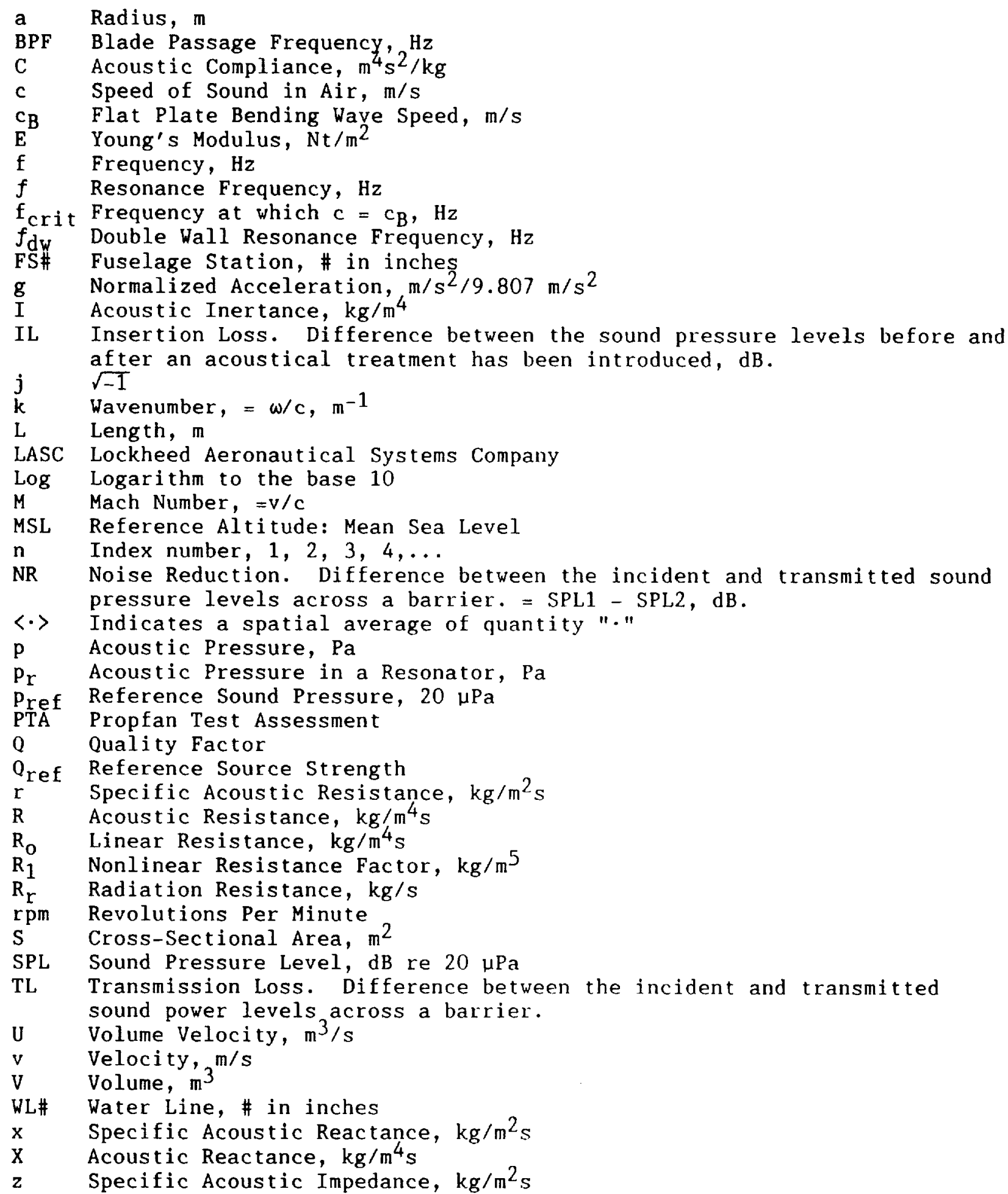




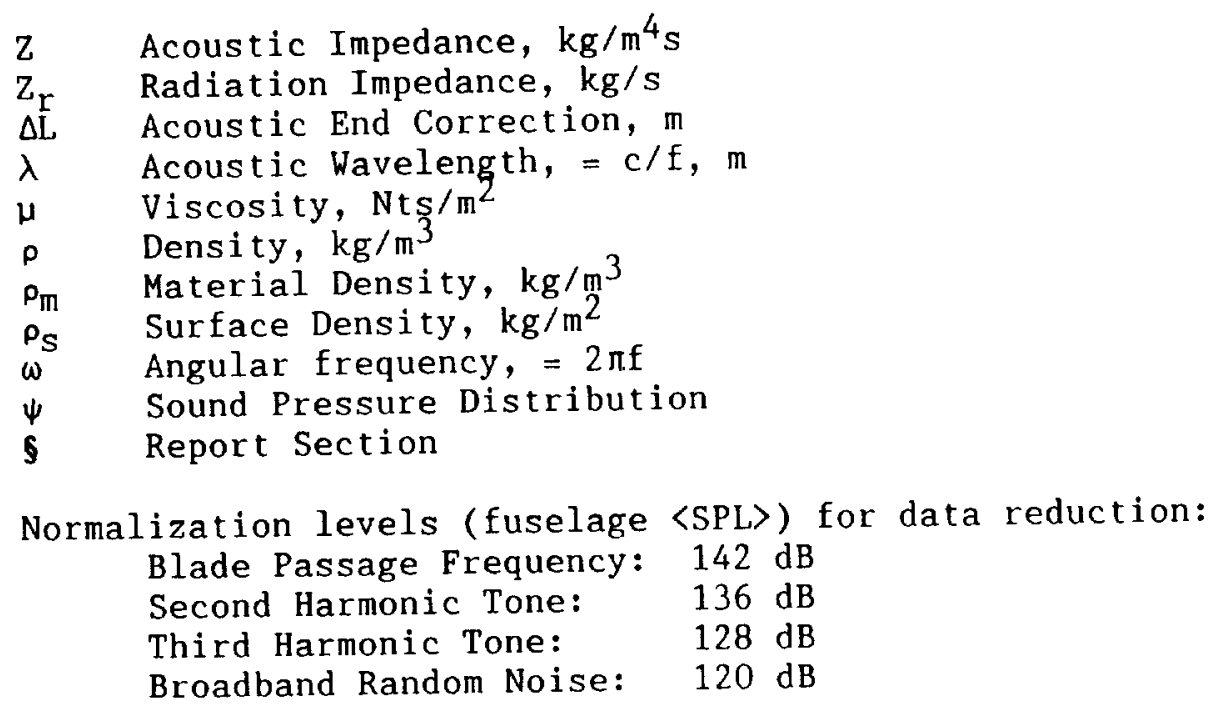


(This is the only text on this page.) 


\section{INTRODUCTION}

\subsection{Summary}

The prime objective of the NASA Acoustic Treatment Technology Program contract with the Lockheed Aeronautical Systems Company (LASC) was to evaluate the acoustic performance of an advanced cabin wall treatment which uses acoustic resonators ${ }^{\star}$ in the Propfan Test Assessment (PTA) Gulfstream II aircraft. This objective was accomplished. Except for flight testing, this program was performed in the Acoustics Laboratory at the Kelly Johnson Research and Development Center at Rye Canyon, Saugus, CA and was funded by the NASALangley Research Center. The present program was awarded in support of the overall PTA program awarded to LASC-Georgia, funded by NASA-Lewis Research Center, and completed in 1989.1,+

As a basis for the wall treatment design, numerous transmission loss measurements were performed in the Acoustics Laboratory. Resonator concepts, which had been developed in the laboratory prior to NASA contractual affiliation on this program, were used in these tests. As a result of this development, an efficient hemispherical Helmholtz resonator design was achieved. In addition, a unique method for rapid resonator and trim panel installation and removal was developed.

A standard Gulfstream II trim panel treatment in which the resonators could be placed was not available. In addition, isolation of the cabin interior from structure-borne noise was considered to be important. As a result, a complete, stand-alone, acoustic enclosure was designed and fabricated. This enclosure was used to simulate the cabin trim in a portion of the PTA aircraft cabin. The enclosure was constructed in California, shipped to Georgia, and installed and flown in the Gulfstream II test aircraft. After the enclosure flight test evaluation, the enclosure was returned to California for subsequent laboratory testing. 2,3 Because of the limited test aircraft availability, the flight test program preceded the laboratory testing.

Seven test flights were performed with the enclosure installed in the PTA cabin at LASC-Georgia. Flight tests were performed at three altitudes, and acoustic measurements were made for a series of propfan rotation speeds. A variety of enclosure and resonator configurations were tested during these flights.

Following the flight test evaluation of the sidewall treatment, tests were performed with a salvaged Gulfstream II fuselage section $(3.49 \mathrm{~m}$ long and 2.39 m diameter) in the Acoustics Laboratory at Rye Canyon. These tests were

\footnotetext{
* A resonator is a device in which it is easy to excite oscillations at one or more discrete frequencies.

+LASC-Burbank contributions to program was summarized in "propfan Test Assessment (PTA) Program Interior Noise Analysis". H.L. Kuntz, Report LR31482, for NASA under contract NAS3-24339 (September 19B8).
} 
performed to enhance our knowledge of the resonator performance with various sidewall configurations.

In order to attenuate multiple harmonic cabin tones generated by the propfan propeller, resonators with multiple resonance frequencies were developed and then tested in an acoustic impedance tube and in the laboratory's transmission loss facility.

\subsection{Background}

Figure 1 summarizes the chronological events and accomplishments relating to the development of cabin sidewall treatments for at tenuating high sound pressure level (SPL) propeller (or propfan) tones within an aircraft cabin. Events, beginning with Lockheed's IRAD studies using Helmholtz resonators within flat double-panel wall assemblies in the mid 1970's and ending with the NASA funded acoustics tasks of the late 1980's and 1990, are outlined in this figure.

In the $1940^{\prime} \mathrm{s}$ propeller manufacturers studied the feasibility of developing radically-swept propeller blades which would permit aircraft to cruise at altitudes and speeds comparable to those achieved by current turbojet and turbofan-powered aircraft. During the late $1940^{\prime} \mathrm{s}$, Hamilton Standard designed and built model swept blades and experimented with supersonic model propellers equipped with various swept blade configurations. Blade flutter and blade stress problems were encountered during these early model tests. These problems resulted in putting this propeller concept on hold. In addition, the noise generated by these propellers was considered an obstacle to their implementation. 4 During this same period, the development of new jet power plants for high-altitude, high-speed cruise overtook propeller development and by the mid 1950's it became apparent that the use of propellers on long-haul aircraft was coming to an end. The commuting public preferred to fly in jetpowered aircraft whose cabins were relatively quiet compared to the propellerpowered DC-7's, Constellations, Electras, and other propeller-driven airliners. The noisy cabins of the latter aircraft were attributed mainly to the propeller blade passage noise striking the fuselage shell. In the 1950's, jet fuel cost was between 10 and 14 cents a gallon, when bought in large quantities. Airlines were not very concerned about the high fuel consumption of early jet-powered airliners like the B-707, DC-8, CV-880, etc. The introduction of the high bypass turbofan power plant for the B-747, with improved fuel utilization over the turbojet, further increased the preference for jet-powered airliners over propeller-powered airliners. In addition to improved fuel utilization, the turbofan's reduced primary jet velocities resulted in a quieter aircraft, both inside and out.

In the 1960's renewed interest was shown in a propulsion system using a sweptbladed unshrouded propeller driven by a jet turbine. Studies showed the feasibility of designing a propeller/turbine power plant capable of cruising at 35,000 feet and speeds of 0.7 to $0.8 \mathrm{M}$. In addition, a large reduction in fuel consumption could be achieved over any of the current jet power plants in existence at the time. Such an improved performance power plant using 
supersonic swept blades was called a propfan power plant to distinguish it from the turbofan or turboprop power plants of the period. 5

Interest heightened in this power plant concept after the large jet fuel cost increases experienced by the airlines during the 1974 oil embargo. Research and development of model propfans by NASA and engine manufacturers accelerated during this period. This resulted in the construction of full scale propfan power plants for testing on various test aircraft. One propeller manufacturer, Hamilton Standard, developed several model propfan blades for laboratory testing at Windsor Locks, CT and at the NASA-Lewis Research Center in Cleveland, $0 \mathrm{H}$. Model propfan testing was accomplished in the $1970^{\prime} \mathrm{s}$ and $1980^{\prime} \mathrm{s}$. In addition, some model propfan flight testing was performed with model propfan power plants mounted on a Lockheed Jet Star aircraft at NASADryden in California.6,7 The structural integrity of these swept blades was demonstrated in various flight environments, and the noise fields generated by these model propfan propellers were defined. These noise data permitted enhanced predictions of noise generated by full scale propfan power plants during flight. This model testing led to the development and construction of a full scale tractor propfan designed for installation on a Gulfstream II aircraft.

During the $1980^{\prime} \mathrm{s}$, successive propfan development led to the SR-7 series of blades, of which the 8-bladed SR-7L was used on the NASA/Lockheed Gulfstream II Propfan Test Assessment (PTA) aircraft.1,8 A sketch of the modified Gulfstream II aircraft is shown in Fig. 2. The propfan fuel efficiency has been estimated to be up to $19 \%$ greater than current turbofan efficiency. 9 The high helical tip speed (up to $1.2 \mathrm{M}$ ) results in intense SPLs at the blade passage frequencies (BPF). Typical fundamental tone SPLs on the fuselage shell were predicted to be as high as $147 \mathrm{~dB}, 10$ with the overall level approaching $150 \mathrm{~dB}$ during cruise flight. 9 These high levels require a greatly increased sidewall transmission loss (TL) at relatively low frequencies (i.e., $225 \mathrm{~Hz}$ ). Large increases in TL usually require sjgnificant additional structural weight, resulting in lower aircraft efficiency. The fundamental tone usually dominates the A-weighted sound levels measured in the cabin of a propeller-driven aircraft. Therefore, an acoustic investigation was conducted to develop a weight-efficient sidewall treatment for use in reducing cruiseflight cabin noise to acceptable levels (between 80 and $85 \mathrm{dBA}$ ). The investigation led to the development of Helmholtz resonators suitable for use within the cabin sidewall cavities formed between the fuselage shell and the interior trim panels.

During the late $1970^{\prime} \mathrm{s}$, the Acoustics Groups of the Lockheed-California Company performed studies and experiments aimed at the development of high transmission loss structures for use in aircraft cabins. 9 The theoretical concepts of Beranek and Work 11 were used to predict TL of simple double-panel assemblies with and without sound-absorptive thermal blankets within the walls. In addition, the theory was extended to include the effects of sidebranch resonators for increasing double-panel wall TL in limited frequency bands.12 In order to demonstrate the validity of the theory, experiments were performed in an impedance tube equipped with a double-panel section which 
included sidebranch resonators between the panels. ${ }^{\star 3}$ Lockheed applied for a patent on this concept and was awarded a patent in 1986.13 others attempted to apply this concept to aircraft panels. 14

During the mid-1980's, Lockheed-supported theoretical and experimental efforts were performed at Rye Canyon. Nonlinear behaviors of resonator nozzles operating in high noise environments were evaluated. +4 others $15,16,17$ have performed work in this area and their work provided a foundation for and confirmation of the Lockheed studies. Lockheed's experimental work was performed using an acoustic impedance tube and a continuous flow resistance measurement apparatus. Some of this work was concurrent with the NASAsponsored development of various Helmholtz resonator configurations for possible installation within the sidewall of an aircraft cabin.

In 1985 NASA-Lewis Research Center awarded PTA Contract NAS3-24339 to the Lockheed-Georgia Company. Lockheed purchased a Gulfstream II aircraft and the Gulfstream Aerospace Corporation plant in Savannah, GA modified the aircraft to accept a single rotor SR-7L tractor propfan power plant on the left wing for full-scale testing. Extensive flight testing was performed to evaluate propfan performance, obtain propfan blade stress data, and evaluate propfan generated acoustic pressures on the aircraft surfaces and in the cabin during various flight conditions. This flight testing was performed in Marietta, GA during 1987. Exterior noise data were measured on the fuselage and on the wing surfaces with a large number of flush-mounted microphones. Internal noise data were measured with a large number of cabin microphones. The test results were reported in 1989.1

In 0ctober 1986, in support of the PTA project, NASA-Langley Research Center awarded Task 3 of Contract NAS1-18036 to the Lockheed-California Company Acoustics Group at Rye Canyon. This contract, and two similar contracts awarded to Boeing Commercial Airplanes 18 and to Douglas Aircraft Company, 19, 20 dealt with the reduction of interior noise levels in propfan-equipped aircraft. Both the Douglas and Boeing aircraft were equipped with fuselagemounted pusher-type counter-rotating propfans.

\footnotetext{
3*"Development of High Transmission Loss structures", L.S. Wirt, D.L. Morrow, and F.J. Balena, Lockheod-California Company, IRAD, LR2B447, volumes I and II $(1978)$.

$4^{+}$"Interior Noise Analysis and Testing of Helmholtz Resonators", L.S. Wirt, Lockheed-California Company, IRAD, LR30976 (December 1985 ) and "Research of High Transmission Loss structures", L.S. Wirt, Lockheed Aeronautical Systems Company - Burbank, IRAD, LR31363 (May 1989).
} 


\section{3 objectives}

The objectives of this program were to:

(1) develop a Helmholtz resonator sidewall acoustic treatment which could significantly increase low frequency cabin TL,

(2) build an acoustic enclosure containing this acoustic treatment for evaluation in the PTA Gulfstream II aircraft, and

(3) develop improvements to sidewall acoustic treatment designs.

\subsection{Significance}

The cabin noise generated by propfan operation limits the use of the propfan configuration until more effective and efficient noise reduction methods can be developed to create a comfortable cabin environment. Significant results have been achieved as a result of this program in developing lightweight, acoustically reactive treatments which show promise for reducing low frequency tonal noise in propfan-equipped aircraft.

With the October 1986 award of the NASA contract to Lockheed, double-panel TL testing and preliminary enclosure design were started. A sub-contract study was awarded to L.D. Pope Associates to incorporate resonators into the Propeller Aircraft Interior Noise (PAIN) computer program.21,22 The PAIN program modifications were completed, predictions were compared with flight test results, and a report and updated program were delivered to Lockheed in September 1988.12

The design, development, and testing of a flight test resonator, shown in Fig. 3 , were completed by August 1987 . Forty-eight tests of noise and vibration reduction by double-panel walls with various resonator and non-resonator configurations were completed in the laboratory TL facility by 0ctober 1987.3

The Gulfstream II aircraft did not have a trim panel configuration which could be modified to support the resonators. Thus, a flight test acoustic enclosure was designed and fabricated. This design was concurrent with the panel tests. Fabrication started in December 1987 and was completed in February 1988. The enclosure was designed with high TL end walls, an aluminum frame support structure, vibration isolation, and interior trim panels, which were designed for quick access to the sidewall resonators. Velcro* -like tapes were used to attach the resonators to the trim panels and the trim panels to the frames. A photograph of this enclosure on its shipping base is shown in Fig. 4. Three of the 28 trim panels (two with 16 resonators each) are in place. One of the heavy high TL end walls can be seen to the left of the enclosure in the photograph. The PTA aircraft is in the background. The enclosure was installed during seven test flights during March 1988 and was returned to California for subsequent laboratory testing.

*Velcro is a registered trademark of Velcro USA INC., Manchester, NH. Velcro and similar materials, manufactured by several companies, were used in this project. 
Laboratory testing of the enclosure was performed in the Acoustics Laboratory at Rye Canyon. 2,3 Testing was performed in the large reverberation room, which was modified to be almost anechoic. Figure 5 is a photograph of the modified reverberation room showing the fuselage section in which the acoustic test enclosure was tested. The laboratory modification and the enclosure tests were performed between August 1988 and September 1989.

Concurrent with the laboratory test program, new resonator concepts were studied. These studies were aimed at attenuating the propfan fundamental and second harmonic tones. During the Summer of 1989 conceptual work started on dual-tuned resonators. Tests and development work started in January 1990. Resonator bench testing and double-panel wall TL testing were completed in August 1990.

Active sound techniques, using small loudspeakers installed within doublepanel wall assemblies, were evaluated very briefly during early 1990 . Such techniques have been used to attenuate exhaust stack fan noise, transformer noise, cabin noise, engine noise, and vibration. No attempt was made to expand this concept for use within aircraft cabin sidewalls, or to determine if such an approach to cabin noise attenuation is feasible or cost effective. The small panel tests were encouraging and this approach is considered worthy of additional study and development.

The report is further divided into the following Sections (\$):

92 A mathematical description of resonators and of sidewall design with resonators.

$\$ 3$ A description of the resonator test results.

$\$ 4$ A description of TL test results on various walls with and without resonators.

$\$ 5$ A description of the acoustic enclosure design.

$\$ 6$ A description of the flight test results with the enclosure in the PTA Gulfstream II aircraft.

$\$ 7$ A description of the laboratory test results with the enclosure in the Gulfstream II fuselage section.

$\$ 8$ A summary of the test program. 


\section{RESONATOR DESIGN}

In this section the design of resonators and their usages in aircraft noise reduction systems are discussed. Tests on individual resonators are described in $\$ 3$ and tests of resonators in double-panel walls are described in $\$ 4$.

\subsection{Concepts of Resonator Usage}

Acoustic resonators have been used for thousands of years to modify acoustic environments.24,25 In recent times they have been used extensively to at tenuate sound at selected frequencies in enclosed spaces, 25 in jet engine nacelles, 26 and in double-panel systems. 13

There are a variety of resonators which may be designed for specific applications. These resonators include: Helmholtz, 27,28 quarter-wavelength, half-wavelength, cascade, 29 waveguide, 30 and combinations of the above. 31,32 Resonators and some applications within aircraft cabins are discussed in this section.

\subsection{In-Panel Resonators}

In order to increase the TL of an enclosure with walls constructed from two panels and an enclosed airspace, resonators and acoustically absorptive materials may be placed between these double-panel walls.11,12,13 An example of the double-wall construction is sketched in Fig. 6. At resonance, the resonators have low impedance and selectively reduce the transmission of sound through the wall. In the case of resonators tuned to multiple frequencies the sound transmission characteristics of the wall are improved at these multiple frequencies.

At frequencies other than resonance, resonators can still influence the double-panel wall TL. Increased nozzle resistance broadens the resonator response bandwidth and lowers the resonator response amplitude. In turn, this change both lowers and broadens the TL increase of a double-panel wall near the resonance frequency. In addition, this acoustically reactive device can reduce the wall TL response at a frequency greater than its resonance frequency. 12,23 This reduction is localized in frequency and is usually offset by the introduction of absorption within the double-panel wall assembly. Absorptive material within the double-panel assembly increases the wall TL at all frequencies, but is less effective at lower frequencies ( $f<300$ $\mathrm{Hz}$ ).

The TL of a flat panel of infinite extent with normal incidence, plane sound waves is 33

$$
\mathrm{TL}=10 \log \left[1+\left(\omega \rho_{\mathrm{S}} / 2 \rho c\right)^{2}\right],
$$

where $\omega=2 \pi \mathrm{f}$, $f$ is the frequency in $\mathrm{Hz}, \rho_{S}$ is the panel surface density in $\mathrm{kg} / \mathrm{m}^{2}, \rho$ is the density of the surrounding fluid, in this case air, and $c$ is the speed of sound in the surrounding fluid. This equation is valid at frequencies below the critical frequency 


$$
\mathrm{f}<\mathrm{f}_{\mathrm{crit}}=\left(\mathrm{c}^{2} / 1.8 \mathrm{t}\right) \sqrt{\rho_{\mathrm{m}} / \mathrm{E}}
$$

where $f_{c r i t}$ is the critical frequency, $t$ is the panel thickness, $\rho_{m}$ is the panel density, and $E$ is the Young's modulus of the panel material. The critical frequency is the frequency at which the speed of sound in air equals the propagation speed of a bending wave in a flat panel. In the region of the critical frequency, panel damping greatly affects the panel TL. 33

When two panels are used to construct a double-panel wall assembly, a resonance condition exists such that the TL is lowered at and near the panel/air/panel system resonance frequency

$$
f_{\mathrm{dw}}=\mathrm{K} / \sqrt{\mathrm{d} \rho_{1} \rho_{2} /\left(\rho_{1}+\rho_{2}\right)},
$$
where $\rho_{1}$ and $\rho_{2}$ are the surface densities of the two panels, $d$ is the panel
spacing, and $K=49.1$ (English), 189 (cgs), and 59.9 (SI). 34,35

The development of equations for calculating the transmission loss of layered walls has been discussed elsewhere, 11,12 and shall be briefly reviewed here. For walls constructed from multiply-layered components, the TL is described by

$$
\mathrm{TL}=20 \log \frac{\mathrm{Z}_{1}+\rho_{\mathrm{o}} \mathrm{c}_{\mathrm{o}}}{2 \mathrm{Z}_{1}} \cdot \frac{\mathrm{p}_{1}}{\mathrm{p}_{2}} \cdot \frac{\mathrm{p}_{2}}{\mathrm{p}_{3}} \cdot \frac{\mathrm{p}_{3}}{\mathrm{p}_{4}} \cdot \frac{\mathrm{p}_{\mathrm{n}}}{\mathrm{p}_{\mathrm{t}}},
$$

where $z_{1}$ is the input impedance of the source side element, $\rho_{0} c_{0}$ is the characteristic impedance of the source side fluid, $p_{1}$ is the sum of the incident and reflected sound pressures, $p_{t}$ is the transmitted sound pressure, and $\mathrm{p}_{\mathrm{n}} / \mathrm{p}_{\mathrm{n}+1}$ is the sound pressure ratio across an element $\mathrm{n}$. The pressure ratio across an element is calculated from the knowledge of the characteristic and termination impedances of the element.11,36

With the introduction of a resonator array 12 into the panel system, the acoustic resonator model needs to be defined. A physical schematic of the system is presented in Fig. 6. The input impedance of a single Helmholtz resonator is

$$
Z_{n}=R_{n}-j\left(\omega I_{n}-1 / \omega C_{n}\right),
$$

where $R_{n}$ is the acoustic dissipation of the $n^{\text {th }}$ resonator, $I_{n}$ is the acoustic inertance, and $C_{n}$ is the acoustic compliance. This array of resonators has a combined acoustic impedance of

$$
\langle Z\rangle=1 / \Sigma\left(1 / Z_{n}\right) \quad \text {. }
$$

The specific acoustic impedance of the resonator array in MKS rayls is

$$
\langle\mathrm{z}\rangle=\mathrm{S}\langle\mathrm{Z}\rangle \quad,
$$


where $S$ is the area of the module array. The specific acoustic impedance of the resonator array acts as a side-branch impedance between the outer and inner panels. The combination of the resonator and receive side, or trim, panel impedances is

$$
\mathrm{Zb}=\langle z\rangle \mathrm{Z}_{\mathrm{tp}} /\left(\langle\mathrm{z}\rangle+\mathrm{Z}_{\mathrm{tp}}\right)
$$

where, in the case of a limp trim panel,

$$
Z_{t p}=\rho_{i} c_{i}-j \omega \rho_{s} .
$$

These equations may be used with Eq. 4 to calculate the system TL. 11 The inclusion of the resonators into the system results in a substantial increase in the TL at the resonance frequency of the resonator. In the case of resonators with multiple resonance frequencies, increases at these multiple frequencies are predicted. In addition to the TL increases, the equations predict a substantial TL decrease at frequencies other than the resonator tuning frequencies. 12 The frequency and magnitude of the decrease depends on the ratio of the double-panel assembly air space volume to the combined volume of the resonators. The reason for this decrease is that a single degree of freedom oscillator (the resonator) is added to a compliant element (sidewall air space) and adds another degree-of-freedom to the system; the combination becomes a "tonraum" oscillator. 37 "This result is most easily determined from a lumped element model including lossless Helmholtz resonators. The Helmholtz resonator impedance is described by

$$
\mathrm{Z}_{\mathrm{r}}=j \omega \mathrm{I}_{\mathrm{r}}+1 / j \omega \mathrm{C}_{\mathrm{r}} \quad \text {, }
$$

where $C_{r}=V_{r} / \rho_{0} c_{o}^{2}$ and $V_{r}$ is the volume of the resonator body. The acoustic impedance of the air space is

$$
\mathrm{Z}_{\mathrm{S}}=1 / \mathrm{j} \omega \mathrm{C}_{\mathrm{S}}
$$

where

$$
C_{S}=V_{S} / \rho_{0} c_{o}^{2}
$$

and $V_{S}$ is the volume of the air space. It is assumed that the wall compliance is much smaller than the air-space compliance, $C_{S}$.

Because the resonator spacing in the sidewall airspace is much less than the resonance frequency wavelength, the summed resonator impedance is

$$
\Sigma Z_{\mathbf{r}}=Z_{r} / N \quad,
$$

where $\mathrm{N}$ is the number of resonators. The impedance of the airspace is in parallel with the resonator impedances. The impedance the source side panel encounters is

$$
Z_{a}=j\left(\omega^{2} I_{r} C_{r}-1\right) /\left[\omega\left(N C_{r}+C_{S}\right)-\omega^{3} I_{r} C_{r} C_{S}\right]
$$


The resonance frequency of the sidewall/resonator combination is merely the resonance frequency of a single Helmholtz resonator in free space

$$
f_{\mathrm{o}}=1 /\left(2 \pi \sqrt{\mathrm{I}_{\mathrm{r}} \overline{\mathrm{C}}_{\mathrm{r}}}\right) \quad \text {. }
$$

On the other hand, the anti-resonances* occur at

$$
f_{X}=0
$$

and

$$
f_{\mathrm{X}}=(1 / 2 \pi) \sqrt{\left(\mathrm{C}_{S}+\overline{N C}_{\mathrm{r}}\right) / \mathrm{C}_{\mathrm{S}} \mathrm{C}_{\mathrm{r}} \mathrm{I}_{\mathrm{r}}}
$$

This last equation may be rewritten as

$$
f_{X}=f_{O} \sqrt{\left(V_{S}+N V_{r}\right) / V_{S}}
$$

\subsection{Illustrations of the Double-Panel Wall Assembly Resonance}

Figure 7 shows the TL versus frequency for three configurations. The TL of a single panel shows a relatively uniform increase in the TL over the frequency range. The double-panel assembly shows a low frequency TL minimum at the double-panel resonance frequency (Eq. 3), and the TL increases more rapidly at the higher frequencies than it does for the single wall. The surface mass of each of the panels in the double-panel structure were taken as half of the single panel surface mass $\left(14.6 \mathrm{~kg} / \mathrm{m}^{2}\right)$. The inclusion of resonators in the space between the panels shifts the double-panel resonance frequency. This shift is caused by changes in the receive side panel surface mass and wall's internal acoustic volume, both caused by the addition of resonators. The large peak is at the mean resonance frequency of the resonator array. The TL minimum at the higher frequency is caused by the interaction of the wall volume compliance and the combined resonator compliances (Eq. 18).

Higher order cross modes detrimentally affect the double-panel TL when these cavity resonances occur as a result of the double-panel cavity edge terminations. These cavity resonance effects are not included in the theoretical predictions presented above. Additional modes occur at higher frequencies where the wall spacing is on the order of $\lambda / 2$, where $\lambda$ is the acoustic wavelength. This latter effect is shown in Fig. 7, where the TL is reduced at the high frequencies. Panel structural modes interact with cavity modes and with resonator response modes to further complicate the TL response of the double-panel system.

\footnotetext{
"Anti-resonances are discrete frequencies at which it is very difficult to excite a resonator or acoustical system. In this case, the second antiresonance occurs because the resonator has added another degree of freedom to the double-panel assembly. The anti-resonance reduces the TL of the double panel wall assembly at this frequency.
} 
Resonators may be tuned to any frequency within the constraints of nozzle and resonator sizes. Part of a resonator array may be tuned to a fundamental frequency and another part may be tuned to a different frequency, such as to the anti-resonance frequency, shown by the $265 \mathrm{~Hz}$ minimum in Fig. 7 . Figure 8 shows the effect of tuning 48 resonators to $234 \mathrm{~Hz}$ and 16 resonators to 265 $\mathrm{Hz}$, the frequency of the anti-resonance (note the vertical scale change to 50 $\mathrm{dB})$. There is a slight drop in the TL at the $234 \mathrm{~Hz}$ resonance frequency, but a substantial rise in the TL at the $265 \mathrm{~Hz}$ resonance frequency. Two new, but lesser, TL minima have replaced the single $265 \mathrm{~Hz}$ minimum, and have been introduced at frequencies just above and below $265 \mathrm{~Hz}$. The additional minimum has been caused by the introduction of another degree-of-freedom into the system.

In addition to the pressure ratio method, described in $\$ 2.2$, a parallel mathematical approach was developed during the present program. This approach is called a transfer matrix method. 12 Comparison to the above approach is favorable. Figure 9 compares the TL predictions obtained from the pressure ratio method and from the transfer matrix method.

\subsection{Resonators within Cabin Space}

When resonators are placed inside the cabin space, they operate as spatial sound absorbers/scatterers. $24,25,36,38$ The absorption of sound in the space depends on the existing passive absorption (e.g., seats and surface coverings) and on the properties of the added resonators (i.e., acoustic dissipation, acoustic reactance, number, and distribution) within the cabin. The NR resulting from the placement of resonators in the cabin is similar to the NR resulting from the placement of resonators in the above-described double-panel module. The exception is that, in the latter case, one must account for the distribution of the resonators and the resulting wave effects.

In order to simplify the acoustical model of how resonators may affect the SPL in an enclosed space, some simplifying approximations were made for the enclosure space. The actual aircraft enclosure is cylindrical with a flat cabin floor which is parallel to the cylinder axis. A rectangular volume of similar dimensions is mathematically simpler to analyze, $38,39,40$ and may be used to illustrate the acoustical effects of adding resonators to a volume. In the following example, the enclosure absorption is chosen to be relatively low. The resonators are assumed to have minimal dissipation, and room mode theory is used for this relatively small room. The impedance seen by a point source in a room is

$$
z_{n}(x, y, z)=\frac{c^{2} \rho \omega \psi_{n}(x, y, z)}{v\left[\left(4 \omega_{n} k_{n}\right)^{2}+\left(\omega^{2}-\omega_{n}^{2}\right)^{2}\right]^{1 / 2}},
$$

where the $n$ subscripts indicate the $n^{t h}$ mode, $k=\omega / c, c=c_{0} \sqrt{T / T_{0}}, c_{0}=331.6$ $\mathrm{m} / \mathrm{s}, \mathrm{T}$ is the absolute temperature, $\mathrm{T}_{0}$ is the reference temperature, $\mathrm{V}=$ LxLyLz is the room volume, and $\mathrm{Lx}, \mathrm{Ly}$, and $\mathrm{Lz}$ are dimensions of the room. The sound pressure distributions in the room for each mode are 


$$
\psi_{n}(x, y, z,)=\cos \left(\frac{n_{x} \pi x}{L_{x}}\right) \cos \left(\frac{n_{y} \pi y}{L_{y}}\right) \cos \left(\frac{n_{z} \pi z}{L_{z}}\right)
$$

The normal frequencies of the room are

$$
\left.f_{n}=(c / 2) \quad \mid \frac{n_{x}}{L_{x}}\right)^{2}+\left(\frac{n_{y}}{L_{y}}\right)^{2}+\left.\left(\frac{n_{z}}{L_{z}}\right)^{2}\right|^{1 / 2},
$$

where $n_{x}, n_{y}$, and $n_{z}$ are integers which index the mode number and $x, y$, and $z$ are the coordinates at the measurement point.

The array of resonators inserted into this room affects the room modes in the vicinity of the resonance frequency. Although this configuration is similar to the resonators in the sidewall, the wave effects are more important here. The resonator influence depends on the impedance and the spatial distribution of the resonators.38 If we consider the modes at the origin $(x=y=z=0)$ and add a weighted-average resonator impedance, $\left\langle\mathrm{Z}_{\mathrm{ra}}\right\rangle$, to the source impedance, then we may approximate the effect of the resonators on the SPL at the origin. From this result we may infer how the sound field is affected in the rest of the enclosure. The equation for the SPL at each frequency becomes

$$
\operatorname{SPL}(0,0,0)=-20 \log \left\{0_{\text {ref }}\left[\left(1 / \Sigma Z_{n}(0,0,0)\right)+\left(1 /\left\langle Z_{\text {ra }}\right\rangle\right)\right] / p_{\text {ref }}\right\},
$$

where $Q_{\text {ref }}$ is the source strength of a tone in the room and $p_{\text {ref }}=20 \mu \mathrm{Pa}$.

Figure 10 shows the approximate effect of Helmholtz resonators $(234 \mathrm{~Hz})$ placed in the room. The dashed curve indicates the SPL measured in the corner of a rectangular room containing moderate surface absorption. The solid curve shows the effects on the SPL with the adrlition of a resonator array having minimal internal dissipation. If the resonators were dissipationless, the attenuation would be concentrated around the resonance frequency. The room modes away from the resonance frequency would not be affected as in Fig. 10 . The measurement position was taken to be at the room origin and all the modes in the frequency range were summed at intervals of $2.5 \mathrm{~Hz}$. If a spatial average had been taken, the modes would not be as dominant as in this figure, and the broad frequency effect of the resonators would not be as obvious. At the resonance frequency the SPL at this position was reduced by $25 \mathrm{~dB}$. Although this is not a rigorous model, it illustrates that the placement of resonators within the cabin reduces the SPL at the resonance frequency.

\subsection{Resonator Types}

A variety of resonator types have been devised and tested under the present program. Photographs of these different resonators are shown in Figs. 3 and 11. The designs of these resonators are discussed in the following paragraphs. 


\subsubsection{Helmholtz}

Classical Helmholtz resonators consist of two components (a nozzle and a volume) which contain three acoustical elements (inertance, compliance, and resistance). Photographs of the flight test resonators are shown in Fig. 3. A sketch of other Helmholtz resonator constructions used in the development program are shown in Fig. 12. The volume is the compliance element and the nozzle, or throat, constitutes the inertance and resistance elements. A resonator in free space has the acoustic impedance of

$$
Z=R_{0}+j(\omega I+1 / \omega C),
$$

where the resistance is $13,41,42$

$$
R_{o}=\left(L^{\prime} / \pi a_{o}^{3}\right) \sqrt{2 \rho \mu \omega},
$$

$I=\rho L^{\prime} / S, C=V_{r} / \rho c^{2}, \rho$ is the fluid density, $\mu$ is the viscosity of air, $L^{\prime}=$ $\mathrm{L}+2 \Delta \mathrm{L}, \Delta \mathrm{L}$ is the end correction, $28,43 \mathrm{~S}=\pi \mathrm{a}_{\mathrm{o}}{ }^{2}$, $\mathrm{a}_{\mathrm{o}}$ is the nozzle radius, and $V_{r}$ is the resonator volume. The value of the end correction may have a variety of values, which depend on the configuration. When $R_{0} \simeq 0$, the resonance frequency is

$$
f_{0}=1 /(2 \pi \sqrt{\mathrm{IC}})
$$

The measurement of resonator amplification is used to describe the frequency response of a resonator. The resonator amplification is the ratio of the acoustic pressure inside the resonator to the acoustic pressure driving the resonator $\left(\mathrm{p}_{r} / \mathrm{p}\right)$.

The peak amplification factor (AF) and the resonator quality factor ( $Q$ ) are related at resonance by

$$
\mathrm{AF}=20 \log (0) \quad \text {. }
$$

In general, the quality factor is defined as

$$
Q=\frac{f_{o}}{\mathrm{f}_{2}-\mathrm{f}_{1}},
$$

where $\mathrm{f} 1$ and $\mathrm{f} 2$ are the frequencies of the half power points, to either side of the resonance frequency, on an amplification response curve. The $Q$ is inversely proportional to the loss factor of an oscillating system. 44

For a lightly damped Helmholtz resonator the quality factor is

$$
Q=\frac{1}{\mathrm{R}} \sqrt{\frac{\mathrm{I}}{\mathrm{C}}}=\left.\frac{\mathrm{pr}}{\mathrm{p}}\right|_{f_{\mathrm{O}}}
$$


For resonators other than Helmholtz resonators, this equation is not applicable. Good design procedure dictates that any dimension of the Helmholtz resonator should be less than $\lambda / 10$, where $\lambda=\mathrm{c} / \mathrm{f}$ at $\mathrm{f}=f_{0}$.

The above equations are valid for low SPLs. At higher SPLs, the nonlinear behavior of the nozzle results in the resonator performance being dependent on the SPL. This results from airflow turbulence in the nozzle, with resulting acoustic resistance increases with SPL. As the resistance increases, the resonator quality factor decreases. The resistance may be divided into two parts, such that the total resistance
may be written as 12,15

$$
\mathrm{R}=\mathrm{R}_{\mathrm{O}}+\mathrm{R}_{1} \mathrm{U} \text {. }
$$

The linear resistance, $R_{0}$, was given in $\mathrm{Eq} .24$ and the nonlinear resistance

$$
R_{1}=\rho /\left(\pi r^{2}\right)^{2} \quad .
$$

Because the resonators are located between the walls and the radiation field is not free for this condition, the radiation resistance term $R_{r}=\rho c k=2 \pi$ has been deleted from Eq. 29.

The rms nozzle flow velocity in a narrow frequency band is

$$
U=\left[\Sigma\left(p_{n}^{2} /\left(R^{2}+x_{n}^{2}\right)\right)\right]^{1 / 2},
$$

where $X$ is the resonator reactance [for a Helmholtz resonator $X_{n}=\omega_{n} I-\left(1 / \omega_{n} C\right)$ ] and $n$ is the summation index over the frequency band. For the case of a single excitation frequency at resonance, $X=0$ and

$$
U=p / R \quad .
$$

In long-throated $\left(\mathrm{L}>\mathrm{a}_{\mathrm{o}}\right)$ resonators the nonlinearity threshold is higher than in short-throated $\left(a_{0}>L\right)$ resonators. 15 For some resonators the nonlinearity threshold may be as low as $100 \mathrm{~dB}$ at standard air conditions.

The next step is to relate the throat resistance to the acoustic pressure and the SPL. The following calculations are performed at the resonance frequency. Combine Eqs. 29 and 32 to obtain

$$
R=R_{0}+R_{1} p / R
$$

In the right hand term the $R$ is still a function of the velocity.

$$
R=R_{0}+R_{1} p /\left(R_{0}+R_{1} U\right)
$$

Through use of the binomial expansion, an approximation to this equation may be written. This approximation is valid for $U R_{1} / R_{0}<1$, and is written 


$$
\mathrm{R}=\mathrm{R}_{\mathrm{o}}+\mathrm{pR}_{1} / \mathrm{R}_{\mathrm{O}}-\left(\mathrm{R}_{1} / \mathrm{R}_{\mathrm{o}}\right)^{2} \mathrm{pU}+\cdots \cdot
$$

The first two terms on the right hand side of Eq. 35 are a first approximation to the resistance relation between the resistance and the acoustic pressure. These terms are equivalent to the substitution of the linear term of Eq. 32 $\left(p / R_{o}\right)$ into $E q .29$. This first approximation assists in approximating the values of $R_{o}$ and $R_{1}$ for use in the Eq. 29, the exact relation.

Although these equations for the nonlinear behavior of resonators have been presented in the context of Helmholtz resonators, the general conclusions should apply to all acoustic resonators described in this project. With increasing SPL each resonator should have an increasing acoustic resistance and a decreasing $Q$.

\subsubsection{Waveguide}

Another resonator developed for evaluation in this program is one termed as a "waveguide" resonator. 30 At the fundamental resonance frequency, this resonator has a length on the order of $\lambda / 10<L_{2}<\lambda / 4$, and has a nozzle at one end. Figure 11 contains a photograph of a waveguide resonator. A sketch of the resonator is shown in Fig. 13. The length of the resonator precludes the use of the Helmholtz resonator equation in calculating the resonance frequency. If the area difference between the body and nozzle is not too large $\left(S_{2}<10 S_{1}\right)$, a good approximation for the impedance of the lossless resonator in free space is

$$
Z=\frac{\rho c}{S_{1}}\left(\frac{\left(\frac{A+1}{A-1}\right)}{\left(\frac{A+1}{A-1}\right)} e^{j 2 k L_{1}+1} e^{j 2 k L_{1}-1}\right),
$$

where $A=j\left(S_{1} / S_{2}\right) \cot \left[k\left(L_{2}-L_{1}\right)\right], L_{1}$ is the nozzle length, $L_{2}$ is the total length, $S_{1}$ is the nozzle cross-sectional area, and $S_{2}$ is the body crosssectional area. The nozzle length, $\mathrm{L}_{1}$, should be increased by a factor of $\Delta \mathrm{L}$, as noted in the Helmholtz resonator equation, above. When the numerator of this equation is zero, the resonator is at a resonance, and the resulting transcendental equation to be solved is

$$
\cot \left[k\left(L_{2}-L_{1}\right)\right]=\left(S_{2} / S_{1}\right) \tan \left(k L_{\cdot}\right) .
$$

When the denominator is zero, the resonator is at an anti-resonance, and the resulting transcendental equation to be solved is

$$
\cot \left[k\left(\mathrm{~L}_{2}-\mathrm{L}_{1}\right)\right]=\left(\mathrm{S}_{2} / \mathrm{S}_{1}\right) \cot \left(\mathrm{kL}_{1}\right) .
$$

In each case, the frequency is determined by finding a value of $k$ which satisfies the equation, since $f=k c / 2 \pi$. Both cases are multi-valued, i.e., 
there are multiple resonances and anti-resonances. These resonances and antiresonances are not harmonically related, and, in some cases, this is advantageous. The frequency ratios of the resonances may be adjusted by modifying the area ratio $\mathrm{S}_{2} / \mathrm{S}_{1}$ and the length ratio $\mathrm{L}_{2} / \mathrm{L}_{1}$.

\subsubsection{Half and Quarter Wavelength Resonators}

The simplest acoustical resonators without nozzles are half wavelength ( $\lambda / 2)$ and quarter wavelength $(\lambda / 4)$ resonators. 28,43 These resonators are related to organ pipes and are easily fabricated. As with the other resonators, the end effect must be included in the calculations. The governing equation for the idealized $\lambda / 2$ resonator acoustic impedance is

$$
Z=(\rho c / S) \sin \left(k L^{\prime}\right)
$$

and the resonance frequencies are

$$
f_{\mathrm{n}}=\mathrm{nc} / 2 \mathrm{~L}^{\prime} \quad, \quad \text { where } \mathrm{n}=1,2,3,4, \ldots \text {, }
$$

$L^{\prime}=L+2 \Delta L, S$ is the cross-sectional area, and $\rho$ is the fluid density. This equation was derived under the assumption that the reflection at each end of the tube involves a complete phase inversion. This assumption is not exact, but suffices as an approximation to the resonator behavior.

The governing equation for the idealized $\lambda / 4$ resonator acoustic impedance is

$$
Z=(\rho c / S) \cot \left(k L^{\prime}\right)
$$

and the resonance frequencies are

$$
f_{n}=[(2 n-1) c] / 4 L^{\prime}, \text { where } n=1,2,3,4, \ldots
$$

and $L^{\prime}=L+\Delta L$. The end correction for this resonator is only half as large as for the $\lambda / 2$ resonator because only one end of the tuhe is open.

\subsection{Double Tuned Resonators}

Double tuned resonators come in a variety of forms. Three forms are discussed in the following paragraphs. Although there are many possible perturbations of shapes and types, the following three resonators were built and tested during the program.

\subsubsection{Double Helmholtz Resonator}

Two Helmholtz resonators may be incorporated into a single structure by a simple modification of the standard flight test resonator. 31 A photograph of this resonator is shown in Fig. 11. The hemispherical resonator is constructed with the nozzle in the vertical position. A truncated cylinder is placed over the hemispherical resonator. A second nozzle is positioned in the side of the cylinder. This construction is shown in the sketch of Fig. 14. The two volumes are independent and both act as individual Helmholtz 
resonators. A disadvantage of this design is that it needs at least two nozzle diameters $(5 \mathrm{~cm})$ of space between the top nozzle and the sidewall insulation above the nozzle. This constraint leads to thicker sidewall construction.

\subsubsection{Double-Tuned Waveguide Resonator}

Two waveguide resonators may be made to resonate at any two frequencies in a single body. 30 The construction saves weight as the end plates are the same for each resonator. In addition, the resonators may be constructed from a single tube. An example of this resonator is shown in the photograph of Fig. 11 and in the sketch of Fig. 15. The resonator in the photograph was constructed from two separate tubes to facilitate ease of fabrication by hand.

\subsubsection{Cascaded Helmholtz Resonator}

Multiple Helmholtz and/or waveguide resonators may be combined to resonate at multiple frequencies and still have only a single nozzle exposed to the outside environment. ${ }^{29}$ An example of this resonator is shown in the photograph of Fig. 11 and in the sketch of Fig. 16. The resonator depicted in these two figures is a double cascaded Helmholtz resonator.

Cascaded resonators are more complicated to analyze than are the other resonators. The cascaded resonator may be constructed from either Helmholtz and/or waveguide resonators. The simplest analysis of the above resonators is performed with cascaded Helmholtz resonators.

The impedance for a lossless, double cascaded Helmholtz resonator is

$$
Z=\frac{\omega^{4} I_{2} C_{2} I_{2} C_{2}-\omega^{2}\left[I_{1}\left(C_{2}+C_{2}\right)+I_{2} C_{2}\right]+1}{\omega^{3} C_{1} I_{2} C_{2}-\omega\left(C_{1}+C_{2}\right)}
$$

The two resonance frequencies are

$$
f_{+}=\frac{1}{2 \pi} \sqrt{\frac{I_{1}\left(C_{1}+C_{2}\right)+I_{2} C_{2} \pm \sqrt{\left[I_{1}\left(\bar{C}_{1}+C_{2}\right)+\bar{I}_{2} C_{2}\right]^{2}-4 I_{1} C_{1} I_{2} C_{2}}}{2 I_{1} C_{1} I_{2} C_{2}}} \cdot 44
$$

The two anti-resonance frequencies are

$$
f_{-}=\sqrt{\frac{\mathrm{C}_{1}+\mathrm{C}_{2}}{2 \pi \mathrm{C}_{1} \mathrm{I}_{2} \mathrm{C}_{2}}} \quad \text { and } \quad f_{-}=0
$$

The inertances and compliances are defined in the section on Helmholtz resonators. The subscripts of the elements refer to the outer (1) and inner (2) resonators.

Waveguide resonators can be made into cascaded resonators. Some measurements on cascaded waveguide resonators were compared to predictions and the 
agreement was poor. Cascaded waveguide resonators were not tested beyond the parameter testing stage. 


\section{RESONATOR TESTS}

The Acoustic Materials Laboratory at Rye Canyon has instrumentation for small scale acoustic resonator and materials testing. Resonators were tested under free-field conditions by the measurement of the transfer function between an external excitation sound field and the resultant resonator internal sound field. The resulting frequency response is called the resonator amplification (see \$2.5.1). Resonator impedance may be measured in the laboratory's standing wave tube at discrete frequencies.

Resonators were tested in order to determine parameter characteristics, refine design equations, and improve the various designs. Tests were performed to determine the acoustical characteristics of nozzle to volume cross-sectional area ratio, resonator nonlinear behavior, resonator size to wavelength, and multiple frequency resonators. The test setup is shown in the photograph of Fig. 17. Free-field high-intensity resonator amplification response was measured in an anechoic room at levels of up to $135 \mathrm{~dB}$. For these highintensity measurements, the small loudspeaker shown in the photograph was replaced by an Emilar driver and horn.

Resonator impedance was measured in an acoustic standing wave (impedance) tube. The nonlinear behavior of resonators was measured at SPLs of up to 145 $\mathrm{dB}$ with this apparatus. A photograph of the standing wave tube system is shown in Fig. 18.

After individual resonators were tested and the designs refined, sets of resonators were fabricated and then evaluated in the TL facility in various flat double-panel wall assemblies (see \$4.2). In a doub]e-panel wall assembly the resonator operation was measured as a function of the surrounding volume, the internal absorption, and the wall TL.

\subsection{Parameter Tests}

Parameter tests were performed on Helmholtz, waveguide, and cascaded resonators. These parameter tests were used to study the effects of wall stiffness, body air leaks, wavelength to body dimension ratio, nozzle length, and nozzle-to-body area ratio.

In each resonator the communication between the compliant element (volume) and the outside should only be through the nozzle. Leaks through the body construction seams are detrimental to resonator operation, as summarized by the data in Fig. 19. The data in this figure were obtained with a $240 \mathrm{~Hz}$ hemispherical Helmholtz resonator. Small holes (1 mm diameter) were drilled in the body to determine the effects of leaks on resonator amplification and tuning. As seen in the figure, increasing the leak area progressively reduces the magnitude of the peak amplification and incleases the resonance frequency.

Another important aspect of resonator design is the acoustic compliance of the structure enclosing the volume. The resonance frequency of Helmboltz resonators with compliant walls cannot be predicted with Eq. 25.12 The effects of wall compliance were measured with the same $240 \mathrm{~Hz}$ Helmholtz 
resonator as used above. The thickness, shape, and materials of the bottom plate were changed and the transfer functions were measured. Four examples are shown in Fig. 20. A $6.3 \mathrm{~mm}$ thick glass plate was used as a standard in these tests. A $0.8 \mathrm{~mm}$ thick concave aluminum bottom was selected for the flight test resonator configuration. The acoustic response of this configuration closely approximates the resonator response of the configuration with the glass plate. When a flat aluminum plate of the same thickness as the curved bottom was used, the resonance frequency and amplification factor were greatly modified relative to the two previous cases. Note that an antiresonance (minimum) appears at about $360 \mathrm{~Hz}$. (The anti-resonances of the two previous cases were not observed because they occurred at frequencies greater than $400 \mathrm{~Hz}$.) In this case the compliance of the resonator has been increased because of the increased compliance of the wall. A further illustration of the effect of wall compliance is the final substitution of a flat $0.3 \mathrm{~mm}$ thick aluminum plate. The resonance frequency was greatly reduced and another resonance and anti-resonance appeared. These results illustrate that, in order to build resonators out of flat-walled boxes, the walls would have to be very stiff (small compliance). On the other hand, cylindrical and spherical resonators have inherently stiff walls and may be designed with thinner walls, with a resulting reduction in mass. Thus, resonator shape and inherent wall stiffness are important to the design of efficient resonators.

The wavelength of sound at resonance relative to the resonator size plays a major role in the manner in which a resonator functions. Parameter tests were performed with resonators made from two cylindrical tubes (48 and $74 \mathrm{~mm}$ I.D.). The resonator volume was changed in each tube by moving a massive piston in the tube. Calculations were compared to measured resonance frequency data. It was determined that while $\lambda>0.1 \mathrm{~L}_{B}$, where $\mathrm{L}_{B}$ is the longest body dimension, the resonance frequency could be accurately calculated with Eq. 25. When $\lambda<0.1 \mathrm{~L}_{\mathrm{B}}, \mathrm{Eq} .25$ became progressively less accurate and the use of Eq. 37 became necessary. Equation 37 was accurate for the whole wavelength range when $S_{2} \geq S_{1} \geq S_{2} / 4$ (see Fig. 13).

When the area ratios between the nozzle and the bodies became too large $\left(S_{1}<S_{2} / 4\right)$, the use of $\mathrm{Eq} .37$ was less accurate in predicting the resonance frequency. In addition, this constraint applied to the calculation of the anti-resonance with Eq. 38. A large area ratio did not appear to affect the accuracy of $\mathrm{Eq} .25$, as long as $\lambda>0.1 \mathrm{~L}_{B}$.

Typical SPL amplification curves of three double-frequency resonators tested during the parameter tests are shown in Fig. 21. Each of the resonators tested had an internal nozzle diameter of $23.6 \mathrm{~mm}$. The nozzle lengths were varied from 9.96 to $49 \mathrm{~mm}$. The resonator volumes were varied from 162 to 2470 $\mathrm{cm}^{3}$. Resonators with the larger volumes tended to exhibit a higher amplification factor, but, because of the increased particle velocity in the nozzle, they also tended to exhibit a lower threshold of nonlinear behavior (see \$3.2 and \$3.3). The band-limited excitation (100 through $500 \mathrm{~Hz}$ ) used during the parameter tests was at an overall SPL of $95 \mathrm{~dB}$.

The resonance frequencies are indicated by the locations of the peaks in the amplification curves. The minimum in the cascaded Helmholtz resonator curve 
indicates the anti-resonance of that resonator. The cascaded Helmholtz resonator (see Fig. 16) response, indicated by the solid line in Fig. 21, shows resonance frequencies of 225 and $455 \mathrm{~Hz}$ and an anti-resonance of $315 \mathrm{~Hz}$. The Helmholtz resonators used during the flight tests (see Fig. 3) had a single resonance frequency at $234 \mathrm{~Hz}$. The high frequency Helmholtz resonator modification (see Fig. 14) added to the hemispherical resonator had a resonance frequency at $455 \mathrm{~Hz}$. The dashed lines of Fig. 21 show the responses of the two Helmholtz resonators. The double waveguide resonator (see Fig. 15) was made from two separate resonators coaxially bonded together into a single body. The two dotted curves of Fig. 21 show the fundamental resonance frequencies to be at $241 \mathrm{~Hz}$ and $422 \mathrm{~Hz}$.

The low frequency waveguide resonator had the highest peak amplification ( 41 $\mathrm{dB}$ ) and the cascaded Helmholtz resonator had the lowest $(29 \mathrm{~dB}$ a $455 \mathrm{~Hz})$. The peak amplifications of all three resonators were quite good, which indicated low nozzle resistances and adequate volumes.

\subsection{Free-Field Measurement of Nonlinear Behavior}

Band limited acoustic excitation (100 through $500 \mathrm{~Hz}$ ) was used during these tests at overall SPLs of: $90,100,110,120,125,130$, and $135 \mathrm{~dB}$. The amplification responses, between the outside and inside of the resonator, were recorded at each SPL. The $Q^{\prime}$ s were calculated by using the built-in software of the 2 channel spectrum analyzer.

The changes in the peak amplification and the quality factors of the resonators versus driving SPL are shown in Figs. 22 and 23 , respectively. As described by Eq. 28, the $Q$ and peak amplification are inversely proportional to the acoustic resistance (dissipation) of the Helmholtz resonator. In addition, the curves of Figs. 22 and 23 indicate that this relation holds true for the other resonator types.

\subsection{Impedance Tube Measurement of Nonlinear Behavior}

The resonators, described in $\$ 3.1$ and $\$ 3.2$, were tested in the impedance tube. Tests were performed at SPLs of: $100,120,125,130,140$, and $145 \mathrm{~dB}$. The impedances were measured at selected frequencies from 200 through $800 \mathrm{~Hz}$, in accordance with the ANSI Standard. 45 The specific frequencies used depended on the individual resonator being tested.

The maximum fluid velocity in the throat occurs at the resonance frequency. It is for this reason that the nonlinearity threshold SPL is the lowest at the resonance frequency. The reactive part of the impedance was determined to be constant with increasing SPL, as shown in Fig. 24. At resonance the reactance values of resonators should be zero $(X=0)$. The range of acoustic reactance values throughout the frequency range for all resonators was between -11 and $6 \rho c$ MKS rayls.

In the case of the low frequency waveguide resonator, there was an error in recording the frequency at which the resonator was tested. Measurements performed before and after the impedance tube tests both showed a resonance 
frequency of $226 \mathrm{~Hz}$. The frequency, recorded as $226 \mathrm{~Hz}$, for this impedance test was not the resonance frequency. Unfortunately, this error was not discovered until all the impedance tests had been completed and the equipment dismantled. This error leads to an erroneous value for the impedance at resonance. From the amplification and $Q$ data (\$3.1 and \$3.2), it was determined that the low frequency waveguide resonator had a lower, not higher, resistance than the flight test resonator.

At and near the resonance frequency, the resistive part of the resonator impedance increases with SPL, as shown in Fig. 25. The resistance increases affect the dissipation, peak amplification, and the effectiveness of the resonator for the present application. The use of a resonator in a doublepanel wall requires that the resonator resistance be as small as possible. This suggests using an array of many resonators, since the array impedance is inversely proportional to the number of resonators (see Eq. 6). Any increase in the resistance adversely affects the resonator performance within a wall assembly. However, resonators placed into the confines of a room to absorb sound should have a moderate amount of absorption, so that some of the acoustic energy may be dissipated in the resonator, rather than being reflected back into the room. 24,25

The cascaded Helmholtz resonator has an anti-resonance between the first two frequencies of interest. This anti-resonance exhibits a decrease in the resistive part of the impedance with SPL increase. This effect is seen in Fig. 26, which shows the resistive part of the impedance versus frequency with SPL as the curve parameter.

As would be anticipated from the results of the impedance data, the resonator absorption coefficient depends on the SPL. In single frequency resonators the maximum change is at the resonance frequency. The absorption coefficient of the resonators at their resonance frequencies versus SPL are shown in Fig. 27. For the cascaded Helmholtz resonator the maximum absorption change was in the region of the anti-resonance $(314 \mathrm{~Hz})$, as shown in Fig. 28 .

The termination of the impedance tube is not perfect $(z \neq \infty)$. The resistive part of the termination impedance ranged between 21 and $53 \rho \mathrm{c}$ MKS rayls. These values are much higher than the values presented in Fig. 25 and results in negligible measurement error. The reactive part was assumed to be zero (this is the method of calibrating the microphone location 45 ). The absorption coefficient of the reference termination ranged flom 8 through $18 \%$, which tends to be smaller than the values presented in Figs. 27 and 28 .

The data in Figs. 22, 23, 25, 26, 27, and 28 show that the nonlinear behaviors of each resonator follow the theoretical conclusions drawn from the discussion in \$2.5. Through the use of Eq. 35, the values of $R_{o}$ and $R_{1} / R_{o}$ were calculated by using a least squares fit to the data in Fig. 25. The values for each resonator are listed in Table I. In addition, the correlation between the data and the $\mathrm{fit}$ is listed for each resonator. An example of this fit to the data is presented for the flight test Helmholtz resonator in Fig. 25 . 
The data fit is excellent for the Helmholtz and the waveguide resonators at all SPLs. The nonlinear behavior of the low frequency waveguide appears to be much greater than for the other resonators. This increased nonlinear behavior may be caused by these measurements being made at an off-resonance frequency, as noted in the discussion of Fig. 24. However, the data presented in Figs. 22 and 23 also show this increased nonlinear behavior.

Although the cascaded resonator appears to follow the same general trend of nonlinear behavior, a better approximation to some of the data might be to use $\sqrt{\mathrm{p}}$, instead of $\mathrm{p}$, for the nonlinear term because the curves tend to flatten with increased SPL. This flattening is seen in Figs. 23 and 25. 
Table I: Table of First Approximations to Resonator Nonlinear Acoustic Flow Resistance Parameters.

$\begin{array}{llll}\text { Resonator Type } & \mathrm{R}_{\mathrm{o}} & \mathrm{R}_{1} / \mathrm{R}_{\mathrm{o}} & \text { Correlation } \\ \begin{array}{l}\text { Hemispherical } \\ \text { Helmholtz } \\ \text { (Flight Test) }\end{array} & 0.122 & 0.0022 & 0.9961 \\ \begin{array}{l}\text { High Frequency } \\ \text { Helmholtz }\end{array} & 0.396 & 0.0018 & 0.9517 \\ \begin{array}{l}\text { Low Frequency* } \\ \text { Waveguide }\end{array} & 0.518 & 0.0049 & 0.9956 \\ \begin{array}{l}\text { High Frequency } \\ \text { Waveguide }\end{array} & 0.025 & 0.0019 & 0.9913 \\ \begin{array}{l}\text { Low Frequency } \\ \text { Cascade }\end{array} & 0.283 & 0.0020 & 0.9280 \\ \begin{array}{l}\text { High Frequency } \\ \text { Cascade }\end{array} & 0.516 & 0.0017 & 0.9756\end{array}$

* A frequency measurement error led to an error in this data, see text. 


\section{DOUBLE-PANEL HALL ASSEMbly TESTS}

\subsection{Transmission Loss Test Facility}

The Acoustics Laboratory consists of anechoic, reverberation, and instrumentation rooms. The rooms are inter-connected such that a variety of tests may be performed. The reverberation room, with inner dimensions of 16.2 $\times 7.9 \times 6.1 \mathrm{~m}(53 \times 26 \times 20 \mathrm{ft})$, was used in conjunction with the anechoic room, with inner dimensions of $5.5 \times 5.5 \times 4.3 \mathrm{~m}(18 \times 18 \times 14 \mathrm{ft})$, to test flat double-panel wall assemblies equipped with acoustic resonators. The reverberation room is connected to the anechoic room by a $1.130 \times 1.130 \mathrm{~m}$ opening in the common wall. A plan view of the two rooms, as set up for TL testing, is sketched in Fig. 29. An isometric sketch of the TL facility in the area of the panel is shown in Fig. 30. A sketch of the clamping mechanism and clamp supports for the wall panels is shown in Fig. 31. A sketch of the wall dimensions and the microphone and accelerometer locations is shown in Fig. 32. This facility is used to test the normal incidence transmission loss of flat wall assemblies. Testing is performed with either random noise or tonal excitation. The SPL differences between the six source and the six receive side microphone pairs are power-averaged to yield a noise reduction (NR) spectrum. Since the receive side is an anechoic room,

$$
\mathrm{TL}=\mathrm{NR}-6 \mathrm{~dB} .
$$

This wall opening allows normal incidence transmission loss testing to be performed on 1.08 by $1.08 \mathrm{~m}$ walls of single or multiple-panel wall construction with wall thicknesses up to $0.165 \mathrm{~m}$. Typical double-panel wall configurations are shown in Fig. 33. The outer (source side) panel of the wall assembly consists of a panel which may be of limp or stiffened construction. When glass fiber insulation batting was installed within double-panel assemblies, the batting usually was in contact with the source side panel. The resonators were usually attacher on the inner surface of the receive side (trim) panel of this assembly. During the labolatory tests, resonator attachment was accomplished by vatious methods. The easiest method for making configuration changes was determined to be with the use of "Velcrolike" tape bonded to the panel surface and to the backs of the resonators. This attachment method was also employed for the flight test configuration. The installation of resonators between the panels of the wall assembly made it possible to define the TL increment achieved with various resonator configurations. The surrounding wall structure is very massive and has a very high TL. The method used to mechanically isolate the source and receive side panels from one another was through the use of closed-cell peripheral seals between the panels and the assembly frame. These seals were $3 \mathrm{~mm}$ thick before being pre-loaded with the panel edge-mounting spring clamps (see Fig. 31).

In the original resonator TL tests, 64 hemispherical Helmholtz resonators, with cylindrical nozzles facing the source panel, were glued to the inner surface of the receive side panel. The panel became the bottom for all the side-by-side resonators. The results of these tests in the flat wall test facility were encouraging and resulted in follow-on testing of various panel 
assembly/resonator configurations. This follow-on work was funded by NASA under the present contract. The results of testing flat panel assemblies with single-frequency Helmholtz resonator arrays are summarized here and in Ref. 23. Forty-eight configurations were evaluated, using different resonator shapes, panel spacings, panel weights, panel dampings, sidewall absorptions, and panel materials. Wall assembly TL tests using single-frequency resonators were performed during the period from February 1987 through October 1987. Similar tests using double-tuned resonators were performed during July and August of 1990. Ten, flat wall and resonator-equipped configurations were tested. Two types of double-tuned resonators were installed and tested in the various double-panel wall configurations.

\subsection{NR Tests of Double-Panel Wall Assemblies with Internal Resonators}

Double-panel wall assembly tests were run on 58 panel/resonator configurations. The assemblies were configured with various surface densities, panel characteristics (damping, stiffness), and additional panel stiffeners. Six resonator types were tested: hemispherical Helmholtz (Types $B$ and $C$ ), integral cylindrical (Type D), cylindrical waveguide (double and single frequency), and double cascaded Helmholtz resonators. See $\$ 2.5$ and $\$ 2.6$ (Figs. 3 and 11 through 16 ) for descriptions of these resonators.

\subsubsection{Single-Tuned Helmholtz Resonators}

Forty-eight of the flat panel wall assemblies, most equipped with various resonator configurations, were used in the evaluation of single-tuned Helmholtz resonators in the TL facility. Figure 34 shows the NR of a typical double-panel (aluminum panels) wall assembly equipped with 64 Helmholtz resonators on the inner surface of the receive side panel. Two NR curves are shown, one with active and one with inactive resonators. At the resonator tuning frequency of $234 \mathrm{~Hz}$, the NR is increased by $13 \mathrm{~dB}$ when the resonators are active. The maximum NR was measured to be $64 \mathrm{~dB}$. Note the NR minimum occurring at approximately $265 \mathrm{~Hz}$ as a result of resonator/panel volume interaction (see \$2.2). This minimum in the NR at $265 \mathrm{~Hz}$ would not be of concern unless a higher frequency excitation tone should coincide with the frequency of the minimum. For this test, no glass fiber batting was installed within the double-panel wall assembly. The data curves are not as smooth as predicted in Fig. 7. The difference results from acoustic cross modes which start at $157 \mathrm{~Hz}$ in the double-panel cavity. This cross mode activity affects the location of the minimum NR, which occurs at $265 \mathrm{~Hz}$ instead of at the anticipated $256 \mathrm{~Hz}$.

Figure 35 shows additional NR data obtained with this wall assembly. This test was performed with Helmholtz resonators tumed to $207.5 \mathrm{~Hz}$. Glass fiber batting was introduced between the panels and bonded to the source side panel.

\footnotetext{
" Noise Reduction Measurements on Double-wall panels with Acoustic Resonators", R.A Prydz, R.J. Gatineau, H.L. Kuntz, and D.L. Morrow, work performed by Lockheed Aeronautical systems Company - Burbank, LR31650, for NASA under Contract NAS1-18036 (April 1989).
} 
This batting cleared the resonator nozzles by approximately $5 \mathrm{~cm}$. The NR at the resonator tuning frequency is affected by the proximity of the batting to the nozzles. With the glass fiber batt addition, the reduction in the peak NR is $3 \mathrm{~dB}$, but at frequencies near the peak NR there is little change. 0ther tests ${ }^{2}$ have shown that if the batting is within one to two nozzle diameters $(2.5$ to $5 \mathrm{~cm})$, the resonator response is affected and the wall assembly NR is significantly reduced at the resonance frequency.

Figure 36 shows the effect on the NR of changing the panel spacing of a double-panel structure with resonators. The source and receive side panels were aluminum and the resonators were installed on the inner wall of the receive side panel. No glass fiber batting was installed between walls for this test. Three different panel spacings were used. The solid curve is for minimum spacing. In this case, the resonator nozzle is less than $11 \mathrm{~mm}$ (less than one-half inch) from the source side panel. This spacing applies to resonators with nozzles centered in the hemispherical body (nozzles at 90 degrees to the panel surface). If this nozzle spacing is further reduced, the resonator performance degrades rapidly. The tuning frequency has shifted to $212.5 \mathrm{~Hz}$ and the $\mathrm{NR}$ to $57 \mathrm{~dB}$. Increasing the panel spacing to $0.165 \mathrm{~m}$, from the minimum spacing of $0.0762 \mathrm{~m}$, causes the NR to increase from 57 to $64 \mathrm{~dB}$ This change introduces a downward shift in peak frequency from $212.5 \mathrm{~Hz}$ to $207.5 \mathrm{~Hz}$. The increase in peak NR is primarily due to increasing the gap between the resonator nozzle exit and the source side panel. The bandwidth of the NR curves at an NR of $55 \mathrm{~dB}$ change from $16 \mathrm{~Hz}(0.076 \mathrm{~m})$ to $24 \mathrm{~Hz}(0.109$ $\mathrm{m})$, to $18 \mathrm{~Hz}(0.165 \mathrm{~m})$. These changes in bandwidth indicate that, although the peak is affected by the wall spacing, the resonators may be effective over a bandwidth which changes little with wall spacing.

At frequencies between the resonance frequency and $300 \mathrm{~Hz}$, a NR minimum, caused by the presence of the resonators, is observed. As the double-panel spacing increases, the frequency at which this minimum occurs decreases. With the smallest spacing of $0.0762 \mathrm{~m}$, this minimum occurs near $263 \mathrm{~Hz}$. With the largest spacing of $0.1651 \mathrm{~m}$, the minimum occurs near $228 \mathrm{~Hz}$. With the intermediate panel spacing of $0.1142 \mathrm{~m}$, the minimum is near $240 \mathrm{~Hz}$. The explanation for these NR minima occurring with resonator activity is discussed in $\$ 2.2$. $^{\star}$

Figure 37 shows the effect of the number of resonators on the NR of a doublepanel aluminum wall structure. One curve shows the NR values versus frequency when all 64 resonators within the wall are active. The second curve shows the result of taping over the nozzles of alternate resonators, deactivating 32 of the 64 resonators. As expected, the NR peak at the average resonator tuning frequency drops by $3 \mathrm{~dB}$, because the overall resistance of the array has doubled (see Eq. 13). The NR peak at the tuning frequency is still substantial with only 32 resonators operating. This result shows that if multiple BPF harmonics were to be attenuated, some of the resonators within

\footnotetext{
*As the wall spacing increases, a point of diminishing return is approached.

As the minimum approaches the peak, the NR of the peak is progressively reduced.
} 
the panel assembly could be tuned to these other harmonics. The effect would be to enhance the panel NR at two or more propfan blade passage frequencies. In addition, if a specific minimum occurred in the NR of a double-panel structure, resonators could be used to reduce the effects of that minimum. *

Figure 38 shows the effect of single- and double-tuned resonators on the NR of a double-panel aluminum wall structure. One curve gives the NR values for the assembly containing 64 active resonators. The NR peak occurs at the resonator tuning frequency of $234 \mathrm{~Hz}$. At $265 \mathrm{~Hz}$ an NR minimum occurs. This minimum is attributed to resonator operation (see $\$ 2.2$ ). It is possible to modify this minimum by introducing a second group of resonators tuned to $265 \mathrm{~Hz}$. Sixteen of the 64 resonators were modified to resonate at $265 \mathrm{~Hz}$ while the rest of the resonators remained tuned at a nominal $234 \mathrm{~Hz}$. The dashed NR curve shows the result of this resonator combination. Note that the NR minimum at $265 \mathrm{~Hz}$ has been eliminated and replaced by an NR peak at $265 \mathrm{~Hz}$. Two new, but less significant minima, have been introduced at frequencies just below and above $265 \mathrm{~Hz}$. This is analogous to the operation of a tuned dynamic absorber in a mechanical system, where side peaks are introduced within the system frequency response when the primary response is altered by the absorber. This test shows the feasibility of mixing different resonators within a panel assembly to achieve multiple tone attenuation, even if the tones are not harmonically related.

Figure 39 shows the effect of using 64 Helmholtz resonators between two 7.32 $\mathrm{kg} / \mathrm{m}^{2}\left(1.5 \mathrm{lb} / \mathrm{ft}^{2}\right)$ limp vinyl panels separated by a $0.165 \mathrm{~m}(6.5 \mathrm{in})$ airspace. These hemispherical resonators were tumerl to $234 \mathrm{Uz}$ and increased the receive side panel surface density to $12.0 \mathrm{~kg} / \mathrm{m}^{2}$. The $11 \mathrm{~dB}$ gain is similar to the 13 $\mathrm{dB}$ gain seen in Fig. 34 with the aluminum panels. The maximum NR was measured to be $62 \mathrm{~dB}$. The double-tuned resonators, discussed in the next section, were tested between these same vinyl panels.

\subsubsection{Double-Tuned Resonators}

Two sets of double-tuned resonators were tested in the TL facility. One set consisted of 38 double waveguide resonators tuned to 241 and $422 \mathrm{~Hz}$. The other set consisted of 52 cascaded Helmholtz resonators tuned to 225 and 455 $\mathrm{Hz}$. These resonators were tested within a wall assembly consisting of two $7.32 \mathrm{~kg} / \mathrm{m}^{2}\left(1.5 \mathrm{lb} / \mathrm{ft}^{2}\right)$ limp vinyl panels separated by a $0.165 \mathrm{~m}(6.5 \mathrm{in})$ airspace. Tests were performed with and without $0.05 \mathrm{~m}$ thick glass fiber insulation attached to the inside surface of the source side panel. TL tests were not run with the double-frequency Helmholtz resonators because, for a practical installation, the low frequency nozzle of this design is too close to the insulation surface for efficient nperation (see Fig. 36).

\footnotetext{
Note that as the number of resonators is reduced, the froquency of the nk minimum, caused by the resonator/airspace intoraction, is also reduced. As the number of resonators approaches zero the poak and minimum approach each other in frequency and NR value, until the rosonator effort vanishes.
} 
The 38 double waveguide resonators were mounted within the limp vinyl doublepanel wall assembly such that the nozzles were not facing each other; half the resonators were placed vertically and half horizontally. Each nozzle was at least $76 \mathrm{~mm}$ from the nearest obstruction. Figure 40 shows three NR data sets for a double-panel wall assembly with the 38 double waveguide resonators installed. The resonator masses increased the surface density of the receive side panel to $15.2 \mathrm{~kg} / \mathrm{m}^{2}$. This surface density is 1.27 times greater than for the case with the Helmholtz (flight test) resonators on the same panel. This increased surface density led to a negligible NR increase (resonators taped) over the 225 through $245 \mathrm{~Hz}$ frequency range. The three test cases were with:

(1) both resonator sections operating,

(2) only the $242 \mathrm{~Hz}$ resonator section operating, and

(3) only the $422 \mathrm{~Hz}$ resonator section operating.

The test results indicate that the $242 \mathrm{~Hz}$ resonators increased the NR by 5.9 $\mathrm{dB}$ and the $422 \mathrm{~Hz}$ resonators increased the NR by $5.5 \mathrm{~dB}$. The maximum NRs were measured to be 59 and $67 \mathrm{~dB}$ at the two lesonance frequencies. As discussed in $\$ 3.1$, the low frequency waveguide resonators have a lower resistive component than the flight test Helmholtz resonators. Even though there were only 38 waveguide resonators, compared to the 64 Helmholtz resonators evaluated during a similar test, it was anticipated that the results would be similar, with the waveguide resonators yielding a NR as much as $2 \mathrm{~dB}$ higher than the flight test resonators. This was not the case. The $64 \mathrm{Helmholtz}$ resonators exhibited better performance, with a $11 \mathrm{~dB}$ NR increase (see Fig. 39). The Helmholtz resonator NR was only $3 \mathrm{~dB}$ greater than the NR with the double-waveguide resonators at the lower resonance frequency.

The 52 cascaded Helmholtz resonators were mounted such that the nozzles all faced upward in four rows of 13 resonators per row. Each nozzle was at least $82 \mathrm{~mm}$ from the nearest obstruction. The resonator masses increased the surface density of the receive side panels to $19.0 \mathrm{~kg} / \mathrm{m}^{2}$. This surface density is 1.58 times greater than the case with the Helmholtz (flight test) resonators at tached to the same panel. This increased surface density led to a measured average NR increase (resonators taped) of $0.2 \mathrm{~dB}$ over the 225 through $245 \mathrm{~Hz}$ frequency range (compare Figs. 39 and 41). Figuce 41 shows two data sets of the resonators operating anr thon inactive (tapeil). At the low frequency resonance the NR increase was $8.7 \mathrm{~dB}$, and at the high frequency resonance the increase was $7.4 \mathrm{~dB}$. This pelfolmance was better than that obtained with the waveguide resonators, but not as good as that obtained with the Helmholtz resonators. The maximum NRs were measured to be 57 and $65 \mathrm{~dB}$ at the two resonance frequencies of 225 and $452.5 \mathrm{~Hz}$, respectively. As seen in $\$ 3.1$ and $\$ 3.2$, the maximum amplifications and $0^{\prime} s$ of the Helmholtz and cascaded resonators were similar. Because of the difference in resonator count (64 to 52) and panel surface densities, it was anticipated that the NR at resonance should have been $2 \mathrm{~dB}$ lower with the cascaded resonators. However, the overall NR of the Helmholtz resonators was $5 \mathrm{~dB}$ higher at the lower resonance frequency.

The flight test Helmholtz resonator was somewhat optimized for a mass-toperformance ratio in these tests. However, in an integral aircraft 
application the resonator/trim panel mass could be further reduced, thus increasing the efficiency of the system. The double-tuned resonators were constructed in an expedient manner without regard to their masses. Through further development, it is anticipated that their masses could be greatly reduced and their acoustical properties enhanced, as was done for the flight test (Helmholtz) resonators.

\subsubsection{Active Sound Control}

As part of the program to increase the TL of double-panel walls, tests using active sound control within the cavity were performed from January through March 1990. Tests were performed with tonal and random excitation at frequencies from 20 to $500 \mathrm{~Hz}$. The separation between the panels of the double-panel assembly was $0.165 \mathrm{~m}$. These tests were performed as a feasibility study and the algorithm used for generating the signal for the control loudspeakers was not optimized for each condition. The same algorithm was used for broadband and tonal signals. In addition, the placements of the loudspeakers and the reference microphones were not optimized. The loudspeakers are used to control the SPL within the total airspace between the walls, and not the motion of the panels directly. Thus, the most uniform sound field is generated by the control loudspeaker when it faces either the source or receive side panel. The results appear to be independent of which panel the loudspeaker faces.

The control loudspeakers were Radio Shack Model 40-2055 loudspeaker units with a $0.12 \mathrm{~m}$ diameter loudspeaker in a box. The integral tweeter loudspeaker was disconnected for these tests. Cross-modes between the panels were recognized as defining a limiting frequency for the effectiveness of active sound control with a single loudspeaker. The first panel cavity cross-morle occurs at 157 $\mathrm{Hz}$. Two types of tests were performed in order to reduce the effects of the cross-modes. For the first configuration, $0.1 \mathrm{~m}$ thick glass fiber batting was placed between the walls with a single loudspeaker unit and one reference microphone. Figure 42 shows the setup from the receive side without the receive side panel in place and without the batting. The single loudspeaker unit is shown on its support stand. For the second configuration, partitions were made to divide the TL facility "window" into four equal and smaller sections. As shown in Fig. 43, each section contained one loudspeaker unit, one reference microphone, and glass fiber batting.

Figure 44 compares receive side SPL spectra at a location $1 \mathrm{~m}$ from the panel. These spectra were measured with and without the single control loudspeaker operating and with the nine, in-phase loudspeaker source array driven with a single frequency electrical input. The reduction in the $100 \mathrm{~Hz}$ input tone SPL was $36 \mathrm{~dB}$ at $100 \mathrm{~Hz}$. Note that reductions occurred at additional frequencies. The active control system tended to flatten the spertrum in the frequency domain, as much as was possible. The control system worker on the background noise spectrum as well as the primary tonal sipnal. The second harmonic tone generated by the source array at $200 \mathrm{~Hz}$ was also attenuated by the active control system. In all tests there were moderate hoadband reductions, and some increases, with the use of the active control. Only the results of the tonal tests are presented here. 
Figure 45 illustrates the effect of active sound control with a single control loudspeaker at discrete, single tone inputs from 20 through $400 \mathrm{~Hz}$. The insertion loss (IL) is calculated as the difference between the NR with the control loudspeaker operating minus the NR with the control loudspeaker off. This calculation ensures that the same source side SPL is used in each calculation. The sound source, which generated plane acoustic waves for the tests, was an array of nine loudspeakers driven in phase. These single tone IL values were measured with the pair of six-microphone arrays described in \$4.1. For the case without the glass fiber batting, the system was tested only up to $260 \mathrm{~Hz}$, because the IL introduced by the active control system was negligible at the higher frequencies. Without glass fiber batts, the highest measured IL was $28.5 \mathrm{~dB}$ at $100 \mathrm{~Hz}$. At $160 \mathrm{~Hz}$ the IL was reduced to $5 \mathrm{~dB}$ because of the influence of the first cross-mode between the panels. The poor performance below $100 \mathrm{~Hz}$ is a result of the low acoustic output of the small control loudspeaker. In the case with the batting in place, the maximum IL was measured to be $20.5 \mathrm{~dB}$ at $80 \mathrm{~Hz}$ and the minjmum IL was measured at $160 \mathrm{~Hz}$. The IL is greater at frequencies above $160 \mathrm{~Hz}$. The presence of the absorptive material reduces the highest value of the IL and broadens the frequency range over which the active sound control system is effective.

Figure 46 shows the results of using a single loudspeaker as a source instead of the nine loudspeaker array. Again, a single control loudspeaker was used. The wave fronts radiated from this loudspeaker approximate spherical rather than planar wave fronts and it was anticipated that the IL would be significantly less with the single source. Examination of Figs. 45 and 46 indicates that, without the glass fiber batts, the 60 through $120 \mathrm{~Hz} \mathrm{IL}$, is greater when the nine loudspeaker array is the source. The 160 and $180 \mathrm{~Hz} \mathrm{IL}$ is greater when the single loudspeaker is used as a source. For the case with the glass fiber batts, the ILs for the two different sources are essentially equivalent. These results lead to the conclusion that the cross-modes between the panels affect both the NR and the IL.

Figure 47 compares the IL resulting from the use of four active control loudspeakers, with single, discrete tone inputs (20 through $400 \mathrm{~Hz}$ ) from the nine loudspeaker array. The signals from the four reference microphones, one for each control loudspeaker, were electronically averaged to provide a single input to the computer. The four control lourspeakers were driven by a single output signal from the computer. The partitions wele installed to increase the frequency of the first cross mode from $157 \mathrm{~Hz}$ to $314 \mathrm{~Hz}$. It was anticipated that the minimum at $160 \mathrm{~Hz}$. in Fig. 45 would be moved to a much higher frequency. The results in Fig. 47 show that this was not the case, as the minimum was moved to $180 \mathrm{~Hz}$. The explanation of this result is not known, but two causes are possible. The first callse may have been that the use of common inputs and outputs from the computer to calculate the noise control signal in each section was not appropriate, even though each of the four panel sections should have the same acoustic input. Phase differences between the four loudspeakers and their respective microphones may have been introduced into the system and this would cause a poor system response. The second cause may have been that the method of partitioning the panel may have caused the panels to vibrate as four independent sections, even though care was taken to avoid this occurrence. The maximum ILs were $22.5 \mathrm{~dB}$ without the glass fiber 
batts and $15 \mathrm{~dB}$ with the glass fiber batts. The enhanced response below 100 $\mathrm{Hz}$ is a result of using four loudspeakers. The reason for the minima in the frequency region of $100 \mathrm{~Hz}$ is unknown. 


\section{PLIGHT TEST ACOUSTIC ENCLOSURE DESIGN}

Concurrent with the panel tests $(\$ 4)$, plans were finalized to evaluate the effectiveness of in-wall (between the cabin trim panels and the fuselage sidewall) resonators in the NASA Gulfstream II PTA aircraft scheduled to be test flown in Marietta, GA. A sketch of the PTA aircraft is shown in Fig. 2. Since this aircraft was untrimmed, a cabin acoustic enclosure was constructed for evaluating the sidewall treatment concept. Flight test scheduling did not allow time for the design and installation of a conventional cabin trim configuration which would have incorporated the test resonators. The decision was made to design and construct an acoustic enclosure that could be shipped to Georgia as an assembly. The acoustic enclosure consisted of a metal framework, plywood end acoustic barriers and floor, removable metal trim panels with removable resonators at tached, and enclosure assembly vibration isolator mountings. Figure 48 is a photograph of this enclosure upon its arrival in Marietta for the flight tests. The trim panels have not been installed, and the enclosure is still attached to a rigid plywood and metal shipping frame, which also acted as an assembly fixture for the enclosure. The assembly fixture ensured that the enclosure-supporting vibration isolators were positioned so as to fit precisely on the existing aircraft cabin seat track rail fittings. The thick plywood end access doors were not installed when this photo was taken. Metal bolts were used instead of rivets to secure all components together, and to permit easy disascembly and reassembly. The propfan, mounted on the Gulfstream II aircraft, can be seen just behind the enclosure framework.

Figure 4 is a photograph showing three of the 28 metal trim panels positioned on the framework. Each panel can accommodate 16 hemispherical resonators. Resonator attachment was accomplished by using Velcro-like fastener tabs bonded to both the panels and the resonator bottoms. A total of 448 resonators $(16 \times 28)$ were used on the trim panels. In the aircraft, 152 additional resonators were installed between the enclosure and cabin floors. These 152 resonators were used to minimize the propfan fundamental tone noise admitted through the enclosure floor. These floor resonators were always active (operating) during the flight and laboratory test programs. The enclosure was installed in the aircraft such that it was approximately centered in the rotation plane of the propfan. Trim panel removal for resonator access was easily accomplished in the ajrcraft since the panels were at tached to the frames with Velcro-like edge strips and cloth sealing tape.

For flight safety reasons, the enclosure structural components, attachments, trim panels and resonator attachments were all stressed to accommodate specified crash loads:

$\begin{array}{cl}\text { Ultimate } & \\ \mathrm{n}_{\mathrm{x}} & -9.0 \mathrm{~g} \text { (forward), } \\ \mathrm{n}_{\mathrm{y}} & \pm 1.5 \mathrm{~g}, \\ \mathrm{n}_{\mathrm{z}} & -2.0 \text { (up), and } \\ & +4.5 \mathrm{~g} \text { (down). }\end{array}$


Various limit design conditions were specified and the stress calculations showed high safety margins for both the ultimate and limit design conditions. The framework of the enclosure was made very rigid to withstand these crash loads. Non-contacting safety fittings were designed to prevent enclosure forward, upward, and sideward motions in the event of a crash. Two safety fittings were installed in seat tracks near the rear of the enclosure, and two between-frame safety fittings were installed at an elevated level at the rear of the enclosure. The 21 vibration isolators securing the cabin to the enclosure floor were selected to accommodate the specified loads, and to minimize the enclosure noise generated by cabin floor vibrations. These stiff rubber isolators (Barry C-2090-T6) supported a test chamber mass of $552 \mathrm{~kg}$ $\left(12141 \mathrm{~b}_{\mathrm{m}}\right)$. This represented an average axial loarl of $26 \mathrm{~kg}(58 \mathrm{lb})$ per isolator. Each isolator can accommodate axjal static loads of $455 \mathrm{~kg}$ (1000 $\left(b_{m}\right)$. Assuming rigid floor points, an average isolator would have a natural frequency of about $40 \mathrm{~Hz}$, which represents a transmissibility of 0.06 at a propfan fundamental frequency of $226 \mathrm{~Hz}$.

Two drawing packages were generated:

(1) Interface Control - PTA Acoustic Enclosure, drawing number 10T0308, FSCM number 98897, job number PTAA2823803, two sheets, $8 / 18 / 87$.

(2) Acoustic Enclosure Assemb]y - PTA Test Bed Acft, drawing number 10T0412, FSCM number 98897, job number PTAA2823B, two sheets, $1 / 28 / 88$.

The frame segments, stringers, and floor beams were constructed of 7075-T6 segments spliced and gusseted together with components of the same material. Figure 49 describes the section properties of the frames, stringers, and floor beams. Figure 50 is a photograph showing circular frames, frame splices, stringers, stringer base plates riveted to stringor hat sections, and frameto-stringer gusset plates. The assembly was in the mocess of being fabricated when this photograph was taken. Figure 51 shows details of the circular frame-to-floor beam junctions with solid steel "hockey stick" load transfer fittings installed within the frame hat sections. The fittings were also used to transfer the vibration momt loads from the cabin floor to the enclosure, and vice versa. Seven vibration isolation mounts were attached to the seven floor frames at each edge of the enclosure, for a total of 14 side mounts. A single vibration isolator is shown in Fig. 51. Seven additional mounts were attached to the center spans of the floor beams. This resulted in a total of 21 mounts securing the seven floor cross beams to the cabin floor seat track fittings. Figure 52 is a photograph showing an installed "kick plate" used as a load transfer plate between frames, and which also acted as an acoustic barrier between the cabin and the enclosure. This $3 \mathrm{~mm}(1 / 8 \mathrm{in})$ thick plate, constructed of 6061-T6 aluminum alloy was installed along each floor edge of the enclosure.

The floorboards were constructed of $19 \mathrm{~mm}$ (3/4 in) thick, 14-ply Finland birch plywood. They were bolted to the floor beams and they overlapped the horizontal surfaces of the kick plates by $88.9 \mathrm{~mm}$ (3.5 in) at each floor edge. These heavy floorboards were used to minimize floor radiated noise. 
The wood end barriers, including the enclosure access doors, were constructed of $12.7 \mathrm{~mm}(1 / 2 \mathrm{in})$ thick, 9-ply Finland birch plywood. These double-panel assemblies were $0.279 \mathrm{~m}$ (11 in) thick and the hollow interiors were filled with glass fiber batting to prevent internal standing acoustic waves from occurring. The inside surfaces of the end walls were covered with a weighted vinyl septum isolated from the plywood surfaces with a $12.7 \mathrm{~mm}(1 / 2$ in) thick layer of glass fiber. This internal treatment added additional acoustic transmission loss to the end walls. The outer peripheral covers of the double-panel barriers consisted of 7075-T6 aluminum alloy sheets $0.254 \mathrm{~m}$ wide by $1.6 \mathrm{~mm}$ thick $(10.0 \times 0.063 \mathrm{in})$ bolted to the two end frames at each end. The plywood components of the enclosure accounted for about half of the total enclosure weight.

The enclosure was sized so that the sidewall resonators cleared the internal flanges of the fuselage frames by $13 \mathrm{~mm}$. Calculated enclosure side deflections under the most severe loading showed that the enclosure would not deflect enough to cause structural interference and impacting of the resonators on the fuselage frames. This $13 \mathrm{~mm}$ clearance also provided space for a $12.7 \mathrm{~mm}(0.5 \mathrm{in})$ thick glass fiber blanket covering of the fuselage frames. Although the tops of the resonators contacted the glass batts at some locations on the frame, the glass was soft enough to vibration isolate the enclosure from the fuselage frame vibrations. 


\section{FLIGHT TESTS}

Flight tests were performed to determine propfan performance and acoustical characteristics with the PTA Gulfstream II aircraft, shown in Fig. 2. Acoustic data were obtained inside and outside a bare, untrimmed cabin during $1987.1,8$ In addition, vibration data were obtained on the wing, fuselage skin, and floor of the cabin.1,8,46 The acoustic enclosure, with sidewall trim and attached resonators, was flown and evaluated in the same Gulfstream II aircraft during March 1988.

\subsection{Untreated Aircraft Tests}

In preparation for the acoustic enclosure flight tests, flight test measurements were obtained in the untrimmed cabin of the PTA aircraft. The objective of the test program was to quantitalively define the paths through which acoustic energy enters the PTA aircraft cabin. The acoustic energy was expected to follow two paths. The first path was assumed to be a direct airborne path from the propfan blades to the fuselage skin and then into the cabin. The second path was assumed to be a structure-borne path from the propfan rotor wake, into the wing, along the wing and fuselage structures, and then radiation into the cabin. Each path was instrumented with appropriate transducers: 32 wing accelerometers, 45 fuselage (internal and external) accelerometers, 44 wing microphones, 18 stationary cabin microphones, 15 cabin microphones on a mobile tram, 45 external fuselage microphones, and 14 airframe strain gages. 1 Figure 53 is a sketch of the cabin microphone designations and locations for the untreated cabin flight test program. A mobile tram with 15 microphones was able to be positioned, in incremental steps, from FS239.5 through FS424 (the MB\#\# microphones in Fig. 53). The propfan rotor was located at FS301. The external instrumentation remained the same for all flight tests, but the cabin instrumentation was different for the flight tests with the enclosure inside the airclaft cabin. Cabin enclosure test instrumentation is described in \$6.\%.

The following two sections contain a symopsis of the data analyzed under the
associated baseline program, which was conducter with an untlimed cabin. 1,8

\subsubsection{Ground Tests}

Acoustic tests were performed with the PTA aircraft in order to determine the relative effects of external fuselage and wing surface propfan tone SPLs on cabin SPLs.1,8 A tonal acoustic signal was introduced in the prop plane of the fuselage with an Emilar loudspeaker and hom combination. The highest tonal SPLs generated at the first three harmonics of the BPF were 124,131 , and $138 \mathrm{~dB}$ re $20 \mu \mathrm{Pa}$, respectively. In addition, measurements were taken at levels which were 6,12 , and $18 \mathrm{~dB}$ lovet than the highest levels. At the highest input SPL, the average harmonic SPLs of the 15 tram microphones at eight tram positions were: $80 \mathrm{~dB}(225 \mathrm{~Hz}), 93 \mathrm{~dB}(450 \mathrm{~Hz})$, and $86 \mathrm{~dB}(675$ $\mathrm{Hz})$. The data were well above the $60 \mathrm{~dB}$ measurement system noise floor.

Additional tests were performed with the acoustic source placed under the wing and inboard of the propfan power plant. The resulting SPLs in the cabin were 
below the electrical noise levels of the cabin acoustic instrumentation. These cabin SPLs were found to be negligible relative to the cabin SPLs resulting from direct acoustic excitation of the fuselage in the propfan plane.

Vibration tests were performed on the PTA aircraft in older to determine the effects of wing vibrations on the cabin SPL. A vibration signal, which was constructed from three tonal components, was introduced into the underside of the wing at three different locations. One location was on the rear spar and inboard of the propfan power plant. The other two locations were on the forward spar, one inboard and one outboard of the propfan power plant. In each case, an Unholtz-Dickie vibration shaker (UD-4C), driven by a power amplifier (TA-250-6-4C), was used to drive the wing. An Endevco force transducer (2104-1000) was used to measure the force input to the wing. This transducer was attached between a plate, which was attached to the wing surface, and the tubular drive rod of the shaker. Four tonal input force levels were generated by the shaker: $45,89,134$, and $178 \mathrm{~N}$ peak. The tonal signal frequencies incorporated the first three harmonics of the propfan BPF: 225,450 , and $675 \mathrm{~Hz}$. One level $(134 \mathrm{~N})$ was used for the vibration tests using broadband excitation signals.

The average acceleration level of the wing was determined to have a linear response relative to the force input. The responses of the cabin microphones were, for the most part, linear relative to the forre input only at the higher force levels. At the lower force levels the hackpround noise levels of the cabin instrumentation influencerl the measuled levels. The focre input at the inboard front spar generated the highest. SPL values in the cabin. The three plots in Fig. 54 show the relative force level input versus the cabin SPL measured at a single location (MBO4) at a tram microphone in the prop plane. The thin straight lines indicate the slope of linear system response. The cabin instrumentation background noise levels were near $60 \mathrm{~dB}$ at 225 and 450 $\mathrm{Hz}$, and near $55 \mathrm{~dB}$ at $675 \mathrm{~Hz}$.

Through the analysis of the above data, relationships were determined for calculating the relative effects of wing acceleration levels and the external fuselage SPLs on cabin SPLs during the flight tests. These data indicated that the predominant path for energy entering the cabin was directly through the fuselage wall. The structural transmission of wing vibrations to the cabin, caused by prop-tip wake impingement and power plant vibration, was determined to be a secondary path for the generation of cabin noise. These relationships were used for some of the cabin SPL predictions discussed in the next section.

\subsubsection{Flight Tests}

Flight tests of the untreated PTA Gulfstream II aicclaft wele performed during altitudes, flight conditions, propfan inflow angles, and power settings. 174 through $237 \mathrm{~Hz}$, which was $77 \%$ through $105 \%$, respectively, of the nominal cruise BPF of $225.6 \mathrm{~Hz}$. The altitudes at which most of the acoustic data were 
taken were, nominally: $1,520,3,660,4,570,8,840$, and $10,700 \mathrm{~m}$ MSL. Atmospheric conditions varied significantly over the period of the flight test program.

The location of the maximum propfan SPL measured on the fuselage varied with condition, but it was generally aft of the propfan rotation plane. The spatial distributions of the first three harmonic tones on the fuselage skin are presented in Fig. 55. A sample spectrum at a single fuselage microphone position is shown in Fig. 56. In addition, the SPL spectrum measured at this fuselage position with the propfan removed is given as a reference in this figure. The reasons for the existence of the low frequency peak with the propfan removed are not known. The corresponding SPL spectra at a single cabin microphone are given in Fig. 57.

Spatial power averages $\left\{\langle\mathrm{SPL}\rangle=10 \log \left[(1 / N) \Gamma\left(p_{n} / \mathrm{P}_{\mathrm{r}} \mathrm{ff}\right)^{2}\right]\right\}$ of the external fuselage skin microphone and internal cabin miccophone signals were taken at a series of BPFs and their corresponding hatmonics. These spatial power averages were used in order to normalize condition-dependent spatial. SPL variations on the fuselage and within the cabin. For these spatial averages the tram microphone array was located in the propfan plane of rotation. Figure 58 shows the $\langle\mathrm{SPL}\rangle \mathrm{s}$ measured on the fuselage skin and in the cabin at six BPFs and at four shaft powers. The helical mach numbers of the propfan blade tips are seen to vary from 0.932 to $1.085 \mathrm{M}$. The $\langle\mathrm{SPL}\rangle$ increases with
helical mach number.

The difference between the external fuselage 〈SPL $\langle\mathrm{SPL}\rangle$ is the spatially-averaged noise reduction, 〈NR, of the aircraft. The differences are shown in Fig. 59 and are seen to be relatively constant over the altitude and BPF frequency ranges. Note that the 〈NR〉 is typically on the order of $25 \mathrm{~dB}$ over the BPF range. In addition, similar 〈NR〉 values were measured at the second and third harmonics of the BPF. ${ }^{8}$

In order to determine the relative input levels of the acoustic and structureborne paths of propfan excitation, the data from the ground tests (\$6.1.1) were analyzed. Data obtained at two altitudes vele compated with corresponding ground test data. Both the fuselape sulface <spt.>s and the spatially-averaged wing acceleration levels ( $\langle A I\rangle$.$) were used in this$ comparison. From the results of these compatisons, predictions of the cabin $\langle S P L\rangle$ for each path were made for the filst three BPF harmonics. Figures 60A and $60 \mathrm{~B}$ are comparisons of the predicted and the measued cabin 〈SPL〉S at these two cruise conditions. The predicted and measured 〈SPL〉 values in the cabin at 10,700 m MSL are shown in Fig. 601. For this comparison, two different spatial averages of the cabin SPI were made. The first spatial average was made with the tram at a single location in the prop plane of rotation. The fifteen tram microphone signals were averaged with all the cabin microphone signals for this average. The second spatial average was made with the tram at the 18 different locations. The predictions are divided according to source. The structure-borne sound from the wing is predicted to generate a much lower 〈SPL〉 than the airborne 〈SPL〉 generated from sound transmitted directly through the fuselage skin. The same information for an altitude of $1,520 \mathrm{~m}$ MSL are presented in Fig. 60B. In this case the measured 
〈SPL values are seen to be much closer to the 〈SPL $\rangle$ values predicted from wing $\langle A L\rangle$ values. The structure-borne generated $\langle\mathrm{SPL}\rangle$ values continue to be much lower than the airborne values at the first two harmonics of the BPF, but are equivalent to the airborne and measured values for the third harmonic of the BPF.

The results of these analyses led to conclusion that that the cabin acoustic signal was dominated by the propfan airborne acoustic signal directly impacting the fuselage. Additional conclusions were that:

(1) the wing vibration and its contribution to cabin noise remains similar at all altitudes;

(2) the acoustic signal input to the fuselage increases with the combination of altitude, propfan power, and helical mach number; and

(3) because of air density and fuselage stiffness effects, cabin pressurization decreases the average 〈NR〉 by $3 \mathrm{~dB}$ at $3,660 \mathrm{~m}$ MSL. 8

These results concerning the effects of structure-borne sound on the cabin 〈SPL are similar to the results of an independent study performed by Unruh. 46

\subsection{Acoustic Enclosure Flight Tests}

A total of seven flights were made from 15 through 24 March 1988 for PTA acoustic test chamber and resonator evaluations. These test flights and related test results are described further in Refs. 3 and 47. *

\subsubsection{Interior Acoustic Treatment}

The interior acoustic treatment used for the evaluation of resollator effectiveness in a flight environment is descuibed in \$5. A trim panel support framework with plywood floor and plyworl acoustic end barriers supported the aluminum alloy trim panels with attached resonators. With the panels in place on the framework, a semi-circular acoustic enclosure resulted which permitted resonator evaluations during flight. The installation of the enclosure in the aircraft cabin formed an annular airspace located between the enclosure surfaces (i.e., trim panels and floor) and the surrounding aircraft surfaces (i.e., fuselage shell and floor). Resonators were placed within this annular airspace, in a way similar to the laboratory double-panel flat wall assemblies tested in the acoustics laboratory TL facility (see \$4). The TL facility panels were surrounded by massive concrete walls, which, essentially, eliminated the flanking noise path around the test panels. On the other hand, the enclosure was located within the high SPL environment of the aircraft cabin. The annular gaps at the ends of the enclnsure were not injtially sealed, and allowed flanking noise to be directly admitted into the annular space containing the resonators.

\footnotetext{
* "Flight Test Cabin Treatment Acoustic Analyajs summary poport propfan Test Ris Aircraft". R.J. Gatineau, Hems company - Rurhank, J.R31730, for NASA under contract NAS1-18036 (september 1989).
} 
During the course of flight testing, this flanking noise problem became obvious and partial peripheral acoustic seals were added to the chamber ends to minimize this noise flanking path. Fulll sealing was not possible because of safety concerns in the event of cabin decompression forward or af $t$ of the enclosure. Gaps were left between the cabin floor and the enclosure floor. Resonator effectiveness was evaluated during flight without and then with these peripheral vinyl and tape end seals. During all test flights, the resonators between floors remained active to supplement the floor TL at the fundamental BPF. The resonator test changes involved only those resonators attached to the twenty-eight trim panels. A total of 600 resonators were installed for these tests, 448 of which were installed between the trim panels and the fuselage shell.

By virtue of the enclosure's rigid and massive construction, most of the cabin noise admitted into the acoustic enclosure was through the trim panels. However, the trim panels with at tached resonators were relatively heavy compared to typical trim panel assemblies. The $1.6 \mathrm{~mm}$ thick aluminum trim panels had a surface density of about $4.6 \mathrm{~kg} / \mathrm{m}^{2}$ with the Velcto-like edge attachment strips. The panels were highly damped because of their method of at tachment to the enclosure framework. The resonators, also equipped with at tachment strips, added another $4.3 \mathrm{~kg} / \mathrm{m}^{2}$ to the panel assembly for a total of approximately $8.9 \mathrm{~kg} / \mathrm{m}^{2}$ total panel surface density. The Velcro-like edge at tachment strips provided considerable damping as did the strips securing the resonators to the trim panel surface. The trim panels did not ring when tapped. The panels with resonators attached constituted an effective acoustic barrier, even with the resonators inactive at the fundamental propfan frequency. 2

This high TL tends to partially conceal the noise attenuation effects of the resonators when the resonators are activated. The schedule did not permit selecting an optimum panel weight prior to flight testing. It is probable that if the panel assembly weight had been significantly less than that tested, the resonator effectiveness would have been more apparent. Still, even with the massive and damped trim panels tested, the resonators were effective in reducing the BPF tone 〈SPL $\rangle$ in the cabin.

The acoustic enclosure was assembled and positionfl within the aircraft cabin so that its center was located close to the propfan plane of rolation at FS301.03, as shown in Fig. 61. The end planes of the enclosure at FS246.88 and FS366.88 are where the partial periphoral vinyl seals were located.

The propfan radiated intense SPLs at the BPF harmonics, the fundamental BPF being $225 \mathrm{~Hz}$ during cruise flight for an engine setting of $100 \%$ RPM. The fundamental BPF was varied from $192 \mathrm{~Hz}$ through $237 \mathrm{~Hz}$ by incremental changes in engine RPM. Flight speed and altitude were kept constant by varying the thrust of the fuselage mounted rear power plants. In order to define the SPL distribution on the fuselage, nineteen microphones were installed on the fuselage surface. Figure 62 shows the microphone locations of the array centered around the propfan plane. The SPIs detected by this microphone array was power-averaged at each of the first seven propfan BPF harmonics. The $\langle S P L\rangle$ S were normalized to account for power plant noise output variations at 
specified test points from flight-to-flight. The difference between the external and internal 〈SPL>s at an RPM setting of the propfan defines the 〈NR〉 for that setting. The individual $\langle N R>s$ at the first seven harmonics were used to compare the enclosure configuration changes from flight to flight.

Figure 63 shows the positions of the microphones installed within the enclosure ( 24 total) and within the wall cavity between the trim panels and the fuselage ( 4 total). In addition, the locations of the three accelerometers used to monitor enclosure frame and floor vibration are shown.

The resonators were tuned to a single resonance frequency of $225 \mathrm{~Hz}$ at an ambient temperature of $0^{\circ} \mathrm{C}$. Since the resonator environment within the sidewall varied from $5^{\circ}$ through $18^{\circ} \mathrm{C}$ during cruise flight, resonator tuning of ten exceeded $230 \mathrm{~Hz}$. This resulted in resonator tuning frequencies being higher than the maximum BPF tone during some cruise flight conditions.

Changes to the enclosure configurations were made between flights. The trim panel configurations were:

1. Resonators active

2. Resonators inactive (taped nozzles)

3. Resonators removed from the trim pane]s

A fourth configuration change, involving the last two flight tests, was the addition of partial vinyl-seals (barriers) to the peripheral (annular) end gaps formed between the enclosure and the fuselage shell.

\subsubsection{Resonator Effects}

Typical fundamental tone $\langle N R\rangle$ s during four identical high altitude cruise flights, three with different trim panel configurations and a fourth without the enclosure, are shown in Fig. 64. With active resonators, the BPF 〈NR〉 of the enclosure/fuselage shell has a peak of about $47 \mathrm{~dB}$ at $232 \mathrm{~Hz}$. With the resonators deactivated, by taping their nozzles closed, the 〈NR〉 drops to about $42 \mathrm{~dB}$, a $5 \mathrm{~dB}$ reduction at $232 \mathrm{~Hz}$. The resonator effect is obvious over a broad BPF range. Removing the resonator masses from the trim panels further reduces the $\langle\mathrm{NR}\rangle$ to about $38 \mathrm{~dB}$ at $232 \mathrm{~Hz}$. This $4 \mathrm{~dB}$ drop is attributed to trim panel mass reduction. The bottom curve of this figure shows the fundamental tone 〈NR〉 of the fuselage shell alone which is approximately $23 \mathrm{~dB}$ at $232 \mathrm{~Hz}$. The acoustic enclosure with active resonators adds another $24 \mathrm{~dB}$ of tone 〈NR> at this frequency which results in a total 〈NR〉 of $47 \mathrm{~dB}$ at 232 $\mathrm{Hz}$. As noted above, if the trim panels and resonators had been considerably lighter, the resonator effectiveness would have been more obvious.

As noted above, the high SPLs in the cabin, forward and aft of the enclosure, were admitted into the wall cavity containing the lesonators through the end peripheral gaps. This intense flanking noise further complicated the evaluation of resonator effectiveness. Figure 6.5 shows the effect on the 〈NR> caused by the reduction in flanking noise by the artlition of the peripheral barrier seals. With the barrier seals in place, the 〈NR〉 exceeded $50 \mathrm{~dB}$ at $232 \mathrm{~Hz}$. With such a barrier seal configuration, the 〈NR〉 increment attributed 
to the enclosure with active resonators exceeds that of the $\langle N R\rangle$ of the pressurized fuselage alone (see Fig. 64).

Typically, in the untreated cabin, the second harmonic 〈SPL〉s were $12 \mathrm{~dB}$ below the fundamental tone $\langle S P L\rangle s$, and the third harmonic $\langle S P L\rangle$ s were $23 \mathrm{~dB}$ below the fundamental tone $\langle S P L\rangle s$ (see Fig. 60). As a result, the high SPL fundamental tone has considerable influence on the A-weighted average noise level within the enclosure. The first seven harmonic tones were used to calculate spatially averaged A-weighted cabin sound levels ( $\langle\mathrm{SL}\rangle$ ). These computed levels are shown in Fig. 66 for three cabin configurations:

(1) Bare interior,

(2) Enclosure with resonators removed, and

(3) Enclosure with active resonators plus end barrier seals.

The target $A$-weighted $\langle S L\rangle$ for the enclosure within the fuselage with active resonators was 80 to $85 \mathrm{~dB}$. At the resonator tuning frequency, the A-weighted $\langle\mathrm{SL}\rangle$ was $86 \mathrm{~dB}$ at the resonance frequency. With some minor refinements, the treatment is expected to operate within the target range. This refinement was one of the goals of the laboratory tests (sfe \$7). These levels are similar to a typical turbofan aircraft's A-weighted interior 〈Si. during cruise flight in the cabin regions of the power plants.

\subsubsection{Altitude Effects on Cabin Noise}

Figure 67 summarizes the fundamental tone $\langle N R\rangle$ s of the fuselage/enclosure combination at three different cruise altitures with active resonators. During a later flight test, the resonators were inactive and the fundamental tone $\langle N R>s$ of this flight are given in Fig. 68 for the same cruise conditions as in Fig. 67. Note that the $\langle N R\rangle$ curves with active resonators are about 6 $\mathrm{dB}$ higher than the $\langle\mathrm{NR}\rangle$ curves with inactive resonators in the fundamental $\mathrm{BPF}$ region above $230 \mathrm{~Hz}$. Based on cabin and resonator temperatures measured during these flights, resonator tuning occurred above $230 \mathrm{~Hz}$. This tuning may account for the 〈NR〉 curves converging at $230 \mathrm{~Hz}$.

At lower BPFs, the low altitude cruise 〈NR>s are significantly less than for the two higher altitude cruise $\langle\mathrm{NR}\rangle \mathrm{s}$. This difference is apparent for both the cases with active and inactive resonators. Since this 〈NR〉 is for the enclosure/fuselage combination, the reduced 〈NR〉 at the lower altitude cruise condition for the lower tone frequencies is likely caused by fuselage pressurization affecting the fuselage modes differently for this lower altitude case. In addition, the increased imperlance of the external air at the lower altitude should decrease the $T L$ of the syctem. 8 While the acoustic enclosure was fairly well sealed acoustically, its trim panels showed no evidence of separating from the enclosure framework because of pressure differential between the cabin and enclosure. Door leakage was likely adequate to equalize the pressure across the enclosure trim panels and no problems were encountered during flight with door opening and closing for chamber access. 


\subsubsection{Vibration Isolation Effects}

The acoustic enclosure was attached to the cabin floor seat tracks with 21 vibration isolators (see \$5). Vibration isolator deactivation or "shorting" provisions existed within nine isolators attached to the three central floor beams. Bottoming out the central bolt of each of these nine isolators ensured solid metal coupling between the enclosure floor beam and the cabin floor seat track.

Figures 69,70 , and 71 show the tone $\langle$ NR $\rangle$ s of the first three harmonics of propfan noise: (1) with 21 active vibration isolators and (2) with nine of the isolators deactivated. Mechanically deactivating nine isolators essentially eliminates the effectiveness of the other 12 isolators. A comparison of the $\langle N R>s$ at the fundamental BPF for the two vibration jsolator configurations (Fig. 69) shows negligible vibration isolation benefit obtained from these nine central isolators.

Figure 70 compares the tone $\langle N R\rangle$ at the second harmonic of the BPF. The active vibration isolator configuration provides more 〈NR〉 than the shorted isolator configuration. The same conclusion can be made for the third harmonic tone (Fig. 71). In each of these two cases the maximum isolation is $2.5 \mathrm{~dB}$. The isolators do have an effect, but not large enough to be measured in the range of the fundamental BPF.

The use of vibration isolators between the enclosure and cabin floors did not significantly affect the 〈NR〉. This is not surprising, in view of the conclusion reached from ground vibration test data obtained on the aircraft (see 6.1). This data indicated that the predominant path for propfan noise entering the cabin was directly from the blades to the cabin wall, and thence, by re-radiation, into the cabin.

\subsubsection{Enclosure Absorption Effects}

Except for the interior end wall surfaces of the enclosure, the interior surfaces were hard surfaces with little sound absorption. The interior end walls were covered with a $0.102 \mathrm{~m}$ thick layer of bagged glass fiber batting to reduce the effects of longitudinal standing waves within the enclosure.

Acoustic data were obtained in the enclosure with and without six $0.305 \mathrm{x}$ $0.305 \times 1.220 \mathrm{~m}(1 \times 1 \times 4 \mathrm{ft})$ plastic foam blocks resting close to the sidewalls to simulate seat and passenger absorption. Three blocks per side were stood on end and were kept in place using the microphone longitudinal support tubes, which extended about $25.4 \mathrm{~cm}$ (10 inches) from the sidewalls. These evenly distributed foam blocks had a total volume of $0.65 \mathrm{~m}^{3}\left(24 \mathrm{ft}^{3}\right.$ ) compared to the enclosure internal volume of $5.4 \mathrm{~m}^{3}\left(192 \mathrm{ft}^{3}\right)$. The total exposed surface area of the foam blocks was $9.2 \mathrm{~m}^{2}\left(102 \mathrm{ft}^{2}\right)$, and this open cell foam had 40 pores per $\mathrm{cm}$ ( 100 per inch).

The effect of introducing the six foam blorks within the enclosure $(100 \%$ absorption) can be seen on the average tone edurtion of the ficst three propfan harmonic tones in Fig. 72, 73, and 74, respertively. The foam blocks 
are not effective in attenuating the fundamental tone, based on the data curves shown in Fig. 72. The effect of added absorption on the $\langle N R\rangle$ s is more obvious at the second and third harmonics, as shown in Figs. 73 and 74.

During most of the tests for determining resonator effectiveness, the six foam blocks were within the enclosure. 


\section{LABORATORY FUSELAGE/ENCLOSURE TESTS}

During 1989 a series of tests was performed on the resonator-equipped acoustic enclosure which was installed in a Gulfstream II fuselage section and located in the Acoustics Laboratory at Rye Canyon. ${ }^{2}$ Figure 5 is a photograph of the fuselage section in the laboratory. The Acoustics Laboratory consists of anechoic, reverberation, and instrumentation rooms. The laboratory fuselage/enclosure tests were performed in order to answer some of the questions raised during the flight tests about resonator operation within the cabin sidewall. Questions raised during the flight tests were:

(1) Why was the resonator effect spread over such a broad frequency range?

(2) Why was the anticipated 〈NR> not achieved?

(3) What was the effect of flanking sound around the ends of the enclosure?

(4) How could the operation of the resonators he improved?

Laboratory tests were performed with two types of acoustic source signals and with numerous variations in the enclosure and resonator configurations. An attempt was made to re-create the flight test acoustic conditions and results. As was the case with the flight tests, any changes made to the resonators did not involve the under-floor resonators. These resonators were operational throughout the laboratory test series.

For the laboratory test program involving the acoustic enclosure, the large reverberation room was made almost anechoic. Glass fiber insulation rolls were installed on the room surfaces, as shown in Fig. 5. The room has inner dimensions of $16.2 \times 7.9 \times 6.1 \mathrm{~m}(53 \times 26 \times 20 \mathrm{ft})$. With the fuselage in place, the average reverberation time is $0.091 \mathrm{~s}$ (range: $0.079-0.101 \mathrm{~s}$ ) in the 100 through $1000 \mathrm{~Hz} 1 / 3$-octave bands. 2 The fusolage shell, shown in Fig. 5 , is $3.49 \mathrm{~m}$ long and $2.39 \mathrm{~m}$ in diameter and was salvagnd from a culfstream II aircraft. The fuselage shell was acoustically exciled with a Ling EPT-94A electro-pneumatic driver attached to an Fimjlar Fil..3 30 acoustic hoin.

The following sub-sections are a synopsis of the laboratory test results which are presented in Refs. 2 and 3.

\subsection{Fuselage Excitation Signals}

Two different acoustic signals were used to excite the fuselage shell during the laboratory tests. The first acoustic signal approximated the propfan signals recorded during the flight tests at a cruise altitude of $10,700 \mathrm{~m}$ MSL. Frequency distribution, spatial distribution, and SPLs were simulated. The fundamental tone was varied in $2 \mathrm{~Hz}$ steps from $225 \mathrm{~Hz}$ to $245 \mathrm{~Hz}$. This range was chosen because the resonators were tuned to $235 \mathrm{~Hz}$ at an average laboratory temperature of $25^{\circ} \mathrm{C}$. The fundamental BPF $\langle\mathrm{SPL}\rangle \mathrm{s}$ measured on the fuselage at three altitudes during the flight tests are compared to the SPLs measured in the laboratory in Fig. 75. A typical laboratory tonal spectrum (fundamental $235 \mathrm{~Hz}$ ) measured on the fuselage near the location of the propeller plane is shown in Fig. 76. Although the spatial SPL distributions 
of the flight and laboratory tests were similar in the region near the plane of the propeller, the SPLs fore and aft of the prop plane were lower in the laboratory. 2

The second acoustic signal used during the laboratory tests was band-limited random noise with an overall level of $138 \mathrm{~dB}$. A typical acoustic spectrum measured on the fuselage in the highest part of the sound field is shown in Fig. 77.

\subsection{Flanking Noise Paths}

During the course of the flight tests, it was determined that the high SPL interior tones in the untreated portions of the cabin forward and af of the enclosure interfered with the evaluation of resonator effectiveness (see \$6.2.1). These sounds may have entered the enclosure through two paths. The first path was around the ends of the enclosure where an annular gap existed. This annular gap was located between the fuselage skin and floor surfaces and the enclosure trim panel and floor surfaces. The second path was through the end walls of the enclosure. The construction of the enclosure was such that it was assumed that this second path was insignificant, because of the inherently high TL of the end barriers.

For the laboratory enclosure/fuselage 〈NR eliminate, as completely as possible, the end flanking path and to further increase the TL of the enclosure end walls. This permitied better measurement of the enclosure/fuselage 〈NR〉 by virtually eliminating the flanking noise admitted into the enclosure.

The end barrier seal treatment consisted of:

(1) adding complete annular and floor gap seals (weighted, limp vinyl) between the fuselage shell section and the enclosure,

(2) adding limp vinyl $\left(9.76 \mathrm{~kg} / \mathrm{m}^{2}\right)$ over the enclosure end walls and door surfaces

(3) adding limp vinyl $\left(4.88 \mathrm{~kg} / \mathrm{m}^{2}\right)$ over the ends of the $3.5 \mathrm{~m}$ long fuselage section, and

(4) installing 50 active resonators in each end cavity formed by the enclosure end wall and the fuselage section end curtain.

This sealing treatment reduced the noise admitted from the enclosure ends to insignificant levels. Prior to adding this end treatment to the enclosure and fuselage shell section, tone levels were measured at the enclosure end walls with the loudspeaker noise source activated. Tone SPLs oil the enclosure end walls and at the peripheral gaps were gonnrally similar to those measured during cruise flight and reported in Refs. ? and 6.

Prior to adding this end wall treatment, fuselage/enclosure tone 〈NR〉 data were obtained with active resonators and with $100 \%$ absorption within the enclosure. These measurements were repeated with the full end treatment configuration. The difference between these two measurements shows the effect of these end treatments on the $\langle\mathrm{NR}\rangle$ of the fundamental tone through the 
fuselage/enclosure sidewall combination. Figure 78 contains curves of 〈NR〉 versus tone frequency for the fundamental tone (resonators active) with and without end barriers in place. These 〈NR〉 curves are similar in level to the fundamental tone 〈NR> levels obtained during cruise flight with and without end peripheral seals. The 〈NR〉 levels of the flight data (Fig. 65) are slightly higher than those of the laboratory data summarized in Fig. 78 . Also, the barrier effect is more obvious during flight, amounting to an 〈NR〉 difference of about $4 \mathrm{~dB}$ at $233 \mathrm{~Hz}$, the approximate resonator average tuning frequency. The 〈NR $\rangle$ difference for the laboratory test data was small, averaging but a few $\mathrm{dB}$ in the frequency band above $230 \mathrm{~Hz}$.

A larger difference had been expected in the laboratory for testing with and without barriers since the flanking noise paths were almost completely eliminated by extensive fuselage/enclosure end sealing.

Figure 79 shows the effect of this end sealing, when the fuselage was excited with band-limited random noise. The 〈NR〉 differential is more like that observed during cruise flight ( $4 \mathrm{~dB}$ typical). Note that the response curves show more of a resonance peak in the laboratory test results than in the flight test results over the 192 through $237 \mathrm{~Hz}$ frequency range (see Fig. 65).

\subsection{Individual Resonator Response}

As part of the tests of resonator operation, individual resonators were instrumented within the sidewall. A trim panel with 16 resonators was modified to hold two microphone probes. One probe was used to measure the acoustic signal in the resonator (resonator probe) and the other probe was used to measure the acoustic signal just outside the resonator (sidewall probe). The panel was mounted on the enclosure frames in the standard fashion at two different locations.

Figure $\mathbf{8 0}$ shows the SPL spectra measured inside the resonator and between the wall panels with the two probe microphones. The morlified trim panel was mounted near mid-cabin on the loudspeaker side of the enclosure. Because of the large data range, the vertical scale in Fig. 80 has been expanded to 100 $\mathrm{dB}$, instead of the usual $50 \mathrm{~dB}$. The sidewall probe shows a deep SPL minimum at the resonance frequency of the resonator artay $(236 \mathrm{~Hz})$. When the resonators are inactive (taped) this minimum disappears. ${ }^{2}$ In addition, there is a minimum (at $242 \mathrm{~Hz}$ ) in the SPL spectrum measured inside the resonator. The minima are not at the same frequencies because of the relative responses of the sidewall and resonators. This latter minimum occurs because:

(1) The resonator amplification is always the same at a specified input SPL and frequency (see $\$ 2.5$ and next paragraph).

(2) Only a limited amount of energy enters the sidewall to excite the airspace at the resonance frequency.

(3) The resonators cancel sidewall sound at the resonance frequency, thus reducing the energy available to excite the resonators in the sidewall at the resonance frequency. 
If these three operations do not occur, then the resonators cannot increase the TL at the resonance Erequency.

Resonator amplification is measured in the same manner in the sidewall as in the laboratory (see \$3); the differences between the curves of Fig. 80 are calculated. In the sidewall case, the acoustic field is in an air cavity, not in free space. The amplification curve for the sidewall resonator mounted at mid-cabin is shown by the solid curve in Fig. 81. The resonance frequency is seen to be at $236 \mathrm{~Hz}$ and the maximum amplification is $42 \mathrm{~dB}$. This maximum amplification is $5 \mathrm{~dB}$ higher than measured in the parameter evaluation tests on a similar Helmholtz (flight test) resonator (see Fig. 21). Next, the modified trim panel was swapped with a trim panel at the aft end of the enclosure on the loudspeaker side of the enclosure. The measured amplification of the resonator at this location is shown by the dashed line in Fig. 81. In this latter case there appears to be interference with the resonator operation because the response peak was shifted to $34 \mathrm{~dB}$ at $238 \mathrm{~Hz}$, an $8 \mathrm{~dB}$ drop from the mid-cabin response. Analysis of these test results contributed to the testing for sidewall insulation interference, which is discussed in $\$ 7.5$.

\subsection{Resonator Effects}

The effectiveness of resonators in reducing cabin enclosure 〈SPL〉s, when attached to the cabin trim panels, was demonstrated in the laboratory in a manner similar to the flight test demonstration.?

Figure 82 shows $\langle N R\rangle_{s}$ of the fuselage and enclosure combination when the assembly was excited with random noise. The three curves show $\langle N R\rangle s$ of three configurations: active resonators, inactive (taped) resonators, and sidewall resonators removed. For these configurations the end barriers and sidewall thermal blankets were installed. Taping the resonators reduced the $\langle N R\rangle$ in the vicinity of the resonance frequency. The peak 〈NR〉 was at $240 \mathrm{~Hz}$ and the maximum 〈NR〉 difference $(9.5 \mathrm{~dB})$ was at $225 \mathrm{~Hz}$. The resonators were found to be effective in the frequency range of 207 through $251 \mathrm{~Hz}$.

The difference in 〈NR〉 levels between active and removed resonator configurations shows the total effect of the resonators installed on the trim panels. In this case, the positive effect is over the frequency range between 150 and $380 \mathrm{~Hz}$. The maximum effect is $18 \mathrm{~dB}$ at $243 \mathrm{~Hz}$. At $424 \mathrm{~Hz}$ the 〈NR〉 was reduced by $8 \mathrm{~dB}$. This reduction is at a frequency below the second harmonic and, for this cabin configuration with propfan excitation, is not considered to be significant.

As illustrated by the tonal test data in Fig. 83, the tonal test results exhibit similar changes in the 〈NR〉 as for lhe three hroadhand excitation cases of Fig. 82. The main difference in these tests is that the tonal test data do not exhibit as sharp a peak in the 〈NR〉 when the resonators are operating, as do the random noise test data. 


\subsection{Thermal Insulation}

Thermal insulation batting is commonly used on the inner surface of the aircraft fuselage skin to reduce the heat flux between the cabin and the external environment. In addition, this insulation acts as an acoustic barrier which limits the entry of fuselage-radiated high-frequency noise into the cabin. Thermal insulation blankets wrapped in Kapton bags were used on the Gulfstream II fuselage ceiling and walls, both during the flight and laboratory tests. The bagged glass fiber blankets were $50 \mathrm{~mm}$ thick. The gap between the fuselage skin and the trim panels was $156 \mathrm{~mm}$. Theoretically, the resonators ( $67 \mathrm{~mm}$ deep) and the thermal blanket combine to fill $118 \mathrm{~mm}$ of this wall space. This is supposed to leave a $48 \mathrm{~mm}$ clearance between the resonator tops and the thermal insulation. It was found that some Kapton bags sagged enough to touch the tops of some resonators, thus reducing some nozzle-to-bag clearances to less than $15 \mathrm{~mm}$. In addition, the typical $76 \mathrm{~mm}$ deep fuselage frames were covered with $13 \mathrm{~mm}$ thick bagged insulation. Some of this insulation was close to some resonator nozzles.

Tests were run to determine what effect thermal insulation had on resonator performance. The end flanking barriers were present throughout these tests. With the resonators active and then inactive, tests were conducted with and without the sidewall insulation installed.

Figure 84 shows the active resonator 〈NR〉 values with the insulation installed and then removed from the sidewalls. The thermal insulation effects were substantial at all frequencies greater than $200 \mathrm{~Hz}$. At the resonance frequency there was minimal change in the $\langle N R\rangle$. When the insulation was removed, the 〈NR > values were reduced at frequencies above and below the resonance frequency. Figure 85 shows changes in the $\langle N R\rangle$ when tonal excitation was used. In agreement with the data in Fig. 84, the changes were small across this limited frequency range.

In order to depict the effect of the sidewall insulation on the resonator function, measurements were made with and without the sidewall insulation present and with inactive resonators. In Fjp. 86 the resonator: 〈IL〉 values are plotted for the two cases (with sidewall insulation and without sidewall insulation). The values for each curve were calculaterl with

$$
\langle\mathrm{IL}\rangle=\langle\mathrm{NR}\rangle \text { active }-\langle\mathrm{NR}\rangle \text { inactive, }
$$

where the subscripts denote the resonator operation. Note that the differences at frequencies above and below the resonance frequency tend to be near $0 \mathrm{~dB}$. Essentially, the effects of the insulating materials were removed from the comparison; the inactive resonator $\langle N R\rangle$ s became the baseline comparisons. The effects of the resonatols under the two conditions become obvious. With the insulation removed, the peak frequency was shifted from 225 to $236 \mathrm{~Hz}$ (the resonance frequency), the $-3 \mathrm{~dB}$ bandwidth of the main peak was reduced from 34 to $20 \mathrm{~Hz}$, and at $236 \mathrm{~Hz}$ the $\langle\mathrm{TL}\rangle$ increased from 7.5 to $11 \mathrm{~dB}$. The minimum at $275 \mathrm{~Hz}$ appears to be a result of the interaction between the resonators and the sidewall acoustical properties (see $\$ 2.2$ ). In the case 
where the insulation was installed, this minimum was eliminated by the absorptive properties of the material.

\subsection{Sidewall/Under-Floor Measurements}

Three microphones were located within the sidewall cavity. A single microphone was located between the enclosure and fuselage floors. The sidewall microphones were located at mid-height (WL113) with one at the front, one at the middle, and one at the rear of the enclosure resonator array on the propfan side. The under-floor microphone was located under the center of the enclosure. These microphones were used to monitor the resonator operation at these different locations.

With the resonators operating, the single point NR between the fuselage 〈SPL〉 and the centrally located sidewall microphone SPL was measured in flight and during the laboratory tests. The four curves in Fig. 87 show tonal NR flight data at three altitudes with tonal laboratory data superimposed. For each of these conditions there were no end barrier seals (the annular gap was open) and the resonators were active.

The under-floor SPL was more affected than the sidewall SPL by the presence of the resonators. The airspace between the floors was $9.5 \mathrm{~cm}$ deep and there was no thermal insulation present. Figure 88 is a comparison of the normalized 〈SPL spectrum of the three sidewall microphones, with and without sidewall insulation, and also the normalized SPL spectrum of the under-floor microphone. In this example, the end barrier seals were installed and the resonators were active. The SPL minimum uncler the floor was at the resonance frequency, whereas the sidewall minimum was at a lower frequency. In addition, the minimum under the floor was $17 \mathrm{~dB}$ lower than the average sidewall minimum. As long as the sidewall insulation was installed, the performance of resonators located near the individual sidewall microphones was not as good as for those located near the under-floor microphone. Removal of the sidewall thermal insulation improved the operation of the sidewall resonators to more closely approach the operation of the under-floor resonators. ${ }^{2}$

\subsection{Resonators in Cabin}

Laboratory tests were performed to show the effectiveness of resonators mounted on the inside surfaces of the trim panels in at tenuating simulated propfan tones within the enclosure. The 〈NR〉 obtained with this configuration was more pronounced at the resonator tuning frequency than with the in-wall configuration. Figure $\mathbf{8 9}$ is a comparison of the 〈NR〉 with the resonators placed inside the sidewall to the $\langle$ NR $\rangle$ with the resonators placed within the enclosure cabin. The $\langle\mathrm{NR}\rangle$ difference of the peak values is almost $5 \mathrm{~dB}$. The effective bandwidth has been reduced from $3 / 4 \mathrm{~Hz}$ (sidfwall resonators) to $7 \mathrm{~Hz}$ (cabin resonators).

From an acoustic viewpoint, locating the resonators inside the cabin seems preferable to installing them out-of-sight within the cabin sidewall. However, internal installation presents problems from a practical viewpoint. 
These problems include safety and aesthetic considerations, as well as acoustical property degradation by passenger and crew tampering. Close to the resonator nozzles the sound field may be higher than without the resonators because of local amplification effects. 48

In their standard positions, the cabin microphones were relatively close to the internally mounted resonators $(9 \mathrm{~cm})$. In order to determine if this microphone proximity enhanced the measured $\langle\mathrm{NR}\rangle$, four microphones were relocated across the cabin for this test only. The microphones were placed at mid-cabin, $1 \mathrm{~m}$ above the floor, and $0.41,0.74,1.22$, and $1.63 \mathrm{~m}$ from the source side enclosure trim panels. At the resonance frequency, the individual cabin microphone NRs (between the fuselage 〈SPL〉 and each fixed cabin microphone SPL) ranged from 47 to $65 \mathrm{~dB}$. The NRs for the four mid-cabin microphones ranged from 54 to $58 \mathrm{~dB}$. This latter range contains the 〈NR〉 value of $56 \mathrm{~dB}$ shown in Fig. 89. The large range of NRs at the individual microphones near the resonators indicate that an internal resonator installation would need to be kept away from passenger ears because of local amplification effects.

\subsection{Vibration}

Vibration tests were performed on the enclosure framework first without and then with the resonator-equipped trim panels. The results of these test are summarized in Ref. 2. The following is a brief summary of the results.

Before the panels were attached to the framework, the frame structure was tap tested to define its vibration responses. Frame vibration modes were found in the region of the fundamental propfan frequency and at higher frequencies.

The enclosure structure was again tested after the panels with resonators had been attached. A Velcro-like tape was used for mounting both the resonators to the panels and panels to the enclosure frame. The attachment of the panels with resonators substantially increased the damping of the frame structure, and modified the modal response.

In order to determine some vibration characteristics of the frame-panelresonator system, vibration tests were performed using a partial frame section supporting a typical resonator-equipped trim panel. This assembly was bolted to a vibration excitation table. Velcro-like tape, as userl in the enclosure, was used to secure the trim panel to the frame section, and to secure the sixteen resonators to the curved aluminum trim panel. The Velcro-like tape was found to be very nonlinear in its response to different driving amplitudes. In addition, the tape vibration characteristics changed with each at tachment. These tests showed that the Velcro-like tape acted as a variable, highly damped, nonlinear spring, and the mounting of the resonators and panels acted as scatter-tuned vibration absorbers. 


\subsection{Recommended Additional Fuselage/Enclosure Testing}

Additional laboratory fuselage tests could include the effects of:

- trim panel stiffness,

- mixing resonator locations both in the cabin and between the sidewalls,

- partial resonator coverage in the sidewalls,

- resonator and trim panel weight optimization, and

- SPL dependent nonlinear behavior of the resonators in the sidewalls.

The results of these tests would allow the fucther refinement of the design of sidewall and cabin treatments using single- and multiple-tumed resonators. We believe that the large stiffness of the fabricated enclosure and the curved aluminum panels partially masked the effect of the resonator operation. The use of soft, vinyl panels (in place of the aluminum panels) would reduce the effect of the panel stiffness on sound transmission. The placement of the resonators in the cabin was found to be successful and fur ther work should study the effect of introducing some resonators into the cabin and leaving the remaining resonators between the sidewalls.

The primary acoustic source acted on a limited area of the fuselage. The effects of partial resonator coverage on this limited area is considered important because of the great weight savings possibilities. Some doubletuned resonators were designed and tested. Resonator design refinements and fuselage testing would be useful in reducing higher harmonic tones in the cabin. The nonlinear behavior of the resonators may have played some role in the performance of the resonators in the flight and laboratory tests at the higher SPL tones. This behavior needs to be explored, because resonators can be designed to be linear over a wider range of Spls. 


\section{CONCLUSIONS}

The work summarized in this report shows that acoustic resonators installed within an aircraft cabin sidewall cavity, formed by the fuselage shell and cabin trim panels, can be used to attenuate propeller tones. The cabin enclosure fabricated for this investigation simulated a cabin trim installation with resonators attached to the outer surfaces of stiff trim panels. The high TL characteristics of the enclosure with inactive resonators tended to partially mask the tone reduction potential of the resonator system. However, the resonator effect was still significant, providing five to six dB of additional tone reduction within the enclosure over a broad frequency range during the flight tests. Through modifications made to the system during the laboratory fuselage tests, it was shown that the additional NR attributed to the resonator operation could be increased to around $11 \mathrm{~dB}$. Had the enclosure TL been less at the fundamental tone frequency, the tone reduction attributed to resonator operation would have been greater.

In evaluating the concept of attenuating propfan tones with resonators, flight and laboratory aconstic tests were performed. In ocder to take advantage of the aircraft availability, the flight tests were performed with the enclosure installed in the PTA Gulfstream II aircraft prior to the laboratory development tests. Optimum scheduling would have placed the flight test effort after the laboratory tests. The PTA aircraft employed a single, wingmounted tractor propfan power plant as the acoustic source. The laboratory tests were performed with the enclosure installed in a Gulfstream II fuselage test section. The laboratory and flight tests were a first at tempt at reducing propfan fundamental tone SPLs in an aircraft cabin by using this inwall resonator concept. The data summarized in this report are adequate for providing guide lines to acoustic designers interested in reducing propeller noise within aircraft cabins.

The enclosure added about $25 \mathrm{~dB}$ of $\langle\mathrm{NR}\rangle$ to the approximately $25 \mathrm{~dB}$ of $\langle\mathrm{NR}\rangle$ obtained by the untreated fuselage shell. The average A-weighted sound level approached $85 \mathrm{~dB}$ during high-altitude cruise flight. This is about $5 \mathrm{~dB}$ higher than a recommended $80 \mathrm{~dB}$ A-weighted sound level. However, with an acoustically refined cabin configuration, it would be possible to achieve this $80 \mathrm{~dB}$ A-weighted sound level. Laboratory tests showed that the cabin fundamental tone SPL reduction could be improved by reducing the interference of the sidewall insulation with the resonator performance, and by adding resonators into the cabin.

In addition to testing hemispherical Helmholtz resonators, special doubletuned resonators were designed, fabricated, and then tested in the flat panel TL facility. The double-tuned resonator tests were feasibility studies and no at tempt was made to optimize the resonator performances. The double-tuned resonator TL performances were not as high as predicted from the parameter measurements. Design refinements are necessary for their efficient use in aircraft.

Another method to control the interior cabin noise levels is through the use of active sound control devices. Tests of this concept were performed in the 
flat panel TL facility. In order to increase the TL of the wall, the control loudspeakers were placed between the panels of the wall. Significant increases in the TL were measured with the operation of the active noise control system at low frequencies. It was determined that the use of glass fiber batts affected the TL at all frequencies. At the frequencies of peak performance, the active control attenuation was reduced by the presence of the sound absorbing batts. At all other frequencies the performance of the active control system was enhanced. This application shows promise and should be studied further. The use of active noise control within the cabin has been studied by other investigators. 49

If aircraft performance studies support the development of an aircraft with propfan power plants mounted on the wing, then new and novel approaches to cabin tone attenuation are needed. The use of sidewall acoustic resonators is one such approach, since a conventional cabin trim configuration may not be acoustically acceptable for such a design. Present-day trim configurations are relatively lightweight and are designed primarily for at tenuating boundary layer noise. Storage compartments, air-conditioning ducts and slots, racks, and galleys, all of which are attached to the fuselage, tend to transmit external noise into the cabin. These short-circuit noise paths all tend to negate or degrade cabin sidewall and ceiling acoustic barrier treatments.

Treatment of the noise at its source would be the most effective solution to this acoustic problem. However, if source treatment is not feasible, then effective fuselage and cabin trim designs will be needed to create an acceptable cabin acoustic environment. Acoustic resonators for noise control within sidewalls is but one of several cabin quieting concepts that has been considered by Lockheed. Other approaches have been studied, e.g., propeller synchro-phasing, fuselage shielding, passive fuselage damping, and fuselage tuned vibration absorbers. One or more of these cabin noise at tenuation methods (including resonators) may be neerled to produce an acceptable cabin noise level. It is recommended that some or all of these methods be studied in the acoustics laboratory with the aim of defining an acceptable cabin noise treatment for an aircraft equipped with wing-mounted propfan or propeller power plants.

Unlike active devices, which tend to be complex and require maintenance, the passive resonators are simple, maintenance-free devices. In order to develop various sidewall treatments of acceptable weight and construction, refinements that are effective in reducing propeller or propfan tones within aircraft cabins to acceptable levels should be studied further. For example, compartmentalized sidewall trim panels with integral resonators could be designed where the trim compartment dimensions are well-defined and would behave acoustically like the flat panel configurations tested in the laboratory TL facility. Multi-tuned trim panel configurations could be developed to attenuate more than one propfan tone within the cabin. Combinations of in-wall and in-cabin resonator: configurations should also be studied. 


\section{REFERENCES}

1. B.H. Little, H.W. Bartel, N.N. Reddy, G. Swift, C.C. Wi thers, and P.C. Brown, "Propfan Test Assessment (PTA) Flight Test Report", NASA CR-182278 (Lockheed Aeronautical Systems Company - Georgia, April 1989) and B.H. Little, D.T. Poland, H.W. Bartel, C.C. Withers, and P.C. Brown, "Propfan Test Assessment (PTA) Final Project Report", NASA CR-185138 (Lockheed Aeronautical Systems Company - Georgia, July 1989).

2. H.L. Kuntz and R.J. Gatineau, "Laboratory Test and Acoustic Analysis of Cabin Treatment for Propfan Test Assessment Aircraft", NASA CR-182075 (May 1991) (Lockheed Aeronautical Systems Company - Burbank, LR31879, NASA Contract NAS1-18036, May 1990).

3. H.L. Kuntz and R.J. Gatineau, "Laboratory Testing of a High Transmission Loss Aircraft Cabin Structure", NOISE-CON 90 Proceedings, 145-151, Austin, TX, 15-17 0ctober 1990 .

4. A.A Regier and H.H. Hubbard, "Status of Research on Propeller Noise and Its Reduction", J. Acoust. Soc. Am. 25:395-404 (1953).

5. F.B. Metzger and T.G. Ganger, "Results of Initial Prop-Fan Model Acoustic Testing, Vol. I - Discussion", NASA CR-111842. (Hamilton Standard, HSER5787, United-Aircraft Company, December 1970).

6. J.H. Dittmar P.L. Lasagna, and K.G. Mackall, "A Preliminary Comparison Between the SR-6 Propeller Noise in Flight and in a Wind Tunnel", NASA TH83341 (1983).

7. J.H. Dittmar, "Further Comparison of Wind Tunnel and Airplane Acoustic Data for Advanced Design High Speed Propeller Models", NASA TM-86935 (1985).

8. H.L. Kuntz and R.A. Prydz, "Interior Noise in the Untreated Gulfstream II Propfan Test Assessment Aircraft", J. of Aircraft 27:647-652 (1990) and AIAA Paper 89-1119, presented at the $12^{\text {th }}$ Aeroacoustics Conference, San Antonio, TX (11 April 1989).

9. R.A. Prydz, J.D. Revell, J.L. Hayward, and F.J. Balena, "Evaluation of Advanced Fuselage Design Concepts for Interior Noise Control on High-Speed Propeller-Driven Aircraft", NASA CR-165960 (Lockheed-California Company, September 1982); and J.D Revel1, F.J. Balena, and R.A. Prydz, "Cabin Noise Weight Penalty Requirements for High-Speed Propfan-Powered Aircraft - A Progress Report", Paper 821360, SAF Nelospace Congress and Exposition, Anaheim, CA (25-28 0ctober 1982).

10. J.H. Dittmar, G.L. Stefpo, and R.J. Jeracki, "Noise of the 10-Bladed $40^{\circ}$ Swept SR-6 Propeller in a Wind Tunnel", NASA TH-82950 (September 1982). 
11. L.L. Beranek and G.A. Work, "Sound Transmission through Multiple Structures Containing Flexible Blankets", J. Acoust. Soc. Am. 21:419-428 (1949).

12. R.A. Prydz, L.S. Wirt, H.L. Kuntz, and L.D. Pope, "Transmission Loss of a Multilayer Panel with Internal Tuned Helmholtz Resonators", J. Acoust. Soc. Am. 87:1597-1602 (1990).

13. L.S. Wirt, "Sound Barrier", U.S. Patent \#4,600,078, (15 July 1986).

14. D.N. May, K.J. Plotkin, R.G. Selden, and B.H. Sharp, "Lightweight Sidewalls for Aircraft Interior Noise Control", NASA CR-172490 (Douglas
Aircraft Company, February 1985).

15. U. Ingard and $\mathrm{H}$. Ising, "Acoustic Nonlinearity of an Orifice", J. Acoust. Soc. Am. 42:6-17 (1967).

16. W.E. Zorumski and T.L. Parrott, "Nonlinear Acoustic Theory for Rigid Porous Materials", NASA TND-6196 (1971).

17. J. Wu and I. Rudnick, "Measurements of the Nonlinear Tuning Curves of Helmholtz Resonators", J. Acoust. Soc. Am. 80:1419-1422 (1986).

18. A.E. Landmann, H.F. Tillema, and S.E. Marshall, "Evaluation of Analysis Techniques for Low Frequency Interior Noise and Vibration of Commercial Aircraft", NASA CR-181851 (Boeing Commercial Airplanes, Contract NAS118027, October 1989).

19. M.A. Simpson, M.R. Cannon, P.L. Burgé, and R.P. Boyd, "Interior Noise Control Ground Test Studies for Advanced Turboprop Aircraft Applications", NASA CR-181819 (Douglas Aircraft Company, Contract NAS118037, April 1989).

20. M.A. Simpson, P.M. Druez, A.J. Kimbrough, M.P. Brock, P.L. Burgé, G.P. Mathur, M.R. Cannon, and B.N. Tran, "UHB Demonstrator Interior Noise Control Flight Tests and Analysis", NASA CR-181897 (Douglas Aircraft Company, Contract NAS1-18037, October 1989).

21. L.D. Pope, E.G. Wilby, and J.F. Wilby, "Propeller Aircraft Interior Noise Model, Part I: Analytical Model", J. Sound Vibration 118:449-467 (1987).

22. L.D. Pope, C.M. Willis, and W.H. Mayes, "Propeller Aircraft Interior Noise Model, Part II: Scale-Model and Flight-Test Comparisons", J. Sound Vibration 118:469-493 (1987).

23. R.A. Prydz, H.L. Kuntz, D.L. Morrow, and L.S. Wirt, "Transmission Loss of Double-Wall Panels Containing Helmholtz Resonators", NOISE-CON 88 Proceedings, 243-248, Purdue University, West Lafayette, IN, 20-22 June
1988 . 
24. V.0. Knudsen \& C.M. Harris, Acoustical Designing in Architecture, (American Institute of Physics, NY, 1978).

25. M.C. Junger, "Helmholtz Resonators in Load-Bearing Walls", Noise Control Eng. J. 4(1):17-25 (1975).

26. E.J. Rice and D.T. Sawdy, "A Theoretical Approach to Sound Propagation and Radiation for Ducts with Suppressors", NASA TM-82612 (1981).

27. H.L.F. Helmholtz, Sensations of Tone, (Dover Publications, Inc. New York, 1954).

28. J.W. Strutt, Baron Rayleigh, The Theory of Sound, Volume II (Dover Publications, New York, 1945).

29. H.L. Kuntz, "Cascaded Helmholtz Resonators to Increase Sound Barrier Transmission Loss", Lockheed Aeronautical Systems Company - Burbank Patent Disclosure \#D-01-7348, 31 August 1989.

30. H.L. Kuntz, "Waveguide Resonators to Increase Sound Barrier Transmission Loss", Lockheed Aeronautical Systems Company - Burbank Patent Disclosure \#D-01-7431, 27 July 1990.

31. D.L. Morrow, Lockheed Aeronautical Systems Company - Burbank Patent Disclosure \#D-01-7348.

32. L.S. Wirt, "Sound-Absorptive Materials to Meet Special Requirements", J. Acoust. Soc. Am. 57:126-143 (1974).

33. I.L. Vér and C.I. Holmer, "Interaction of Sound Waves with Solid Structures", in Noise and Vibration Control, L.L. Beranek, Ed., (McGrawHill, Inc., New York, $\overline{1971) . ~}$

34. P.A. Franken, E.M. Kerwin, Jr., and BBN Staff, "Methods of Flight Vehicle Noise Prediction", Volume 1, Wright Air Development Center, Air Research and Development Command, United States Air Force, WADC Technical Report 58-343 (Part 1), AD205776, November 1958.

35. B. Feder, "Mass-Air-Mass Resonance Nomograph", Sound and Vibration 4(8): 25 (1970).

36. C. Zwikker and C.W. Kosten, Sound Absorbing Materials, (Elsevier Publishing Company, Inc., New York, 1949).

37. I. Malecki, Physical Foundations of Technical Acoustics (Pergammon Press, New York, 1969).

38. P.M. Morse and K.U. Ingard, Theoretical Acoustics, (McGraw-Hill Book Company, New York, 1968). 
39. L.L. Beranek, "Sound in Small Spaces", in Noise and Vibration Control, L.L. Beranek, Ed., (McGraw-Hill, Inc., New York, 1971).

40. T.F.W. Embleton, "Sound in Large Rooms", in Noise and Vibration Control, L.L. Beranek, ed., (McGraw-Hill, Inc., New York, $1 \overline{971})$.

41. I.B. Crandall, Theory of Vibrating Systems and Sound, (D. Van Norstrand Company, Inc., Princeton, $\overline{\mathrm{NJ}}$ ).

42. R.H. Bolt, L.L. Beranek, and R.B. Newmann, "Handbook of Acoustic Noise Control", Vol. 1, WADC 52-204 (1952).

43. L.E. Kinsler and A.R. Frey, Fundamentals of Acoustics, Second Edition (John Wiley \& Sons, Inc. New York, 1962).

44. E.E. Ungar, "Damping of Panels", in Noise and Vibration Control, L.L. Beranek, ed., (McGraw-Hill, Inc., New York, 1971).

45. ANSI/ASTM C384-77, "Standard Test Method for Impedance and Absorption of Acoustical Materials by the Impedance Tube Method" (American National Standards Institute, New York, 1977).

46. J.F. Unruh, "Structure-Borne Noise Estimates for the PTA Aircraft", Southwest Research Institute, NASA CR-4315 (1990).

47. H.L. Kuntz, R.J. Gatineau, and R.A. Prydz, "Acoustic Transmission Loss Flight Test Results for an Aircraft Cabin Enclosure", INTFR-NOISE 89 Proceedings, 211-216, Newport Beach, CA, 4-6 December 1989.

48. K.U. Ingard, "The Near Field of a Helmholtz Resonator Exposed to a Plane Wave", J. Acoust. Soc. Am. 25:1062-1067 (1953).

49. M.A. Simpson, T.M. Luong, M.A. Swinbanks, M.A. Russel, and H.G. Leventhall, "Full Scale Demonstration Tests of Cabin Noise Reduction Using Active Noise Control", INTER-NOISE 89 Proceedings, 459-462, Newport Beach, CA, 4-6 December 1989. 
LEGEND: $\square$ Reports $\triangle$ Papers/Presentations $\nabla$ Patents/Disclosures

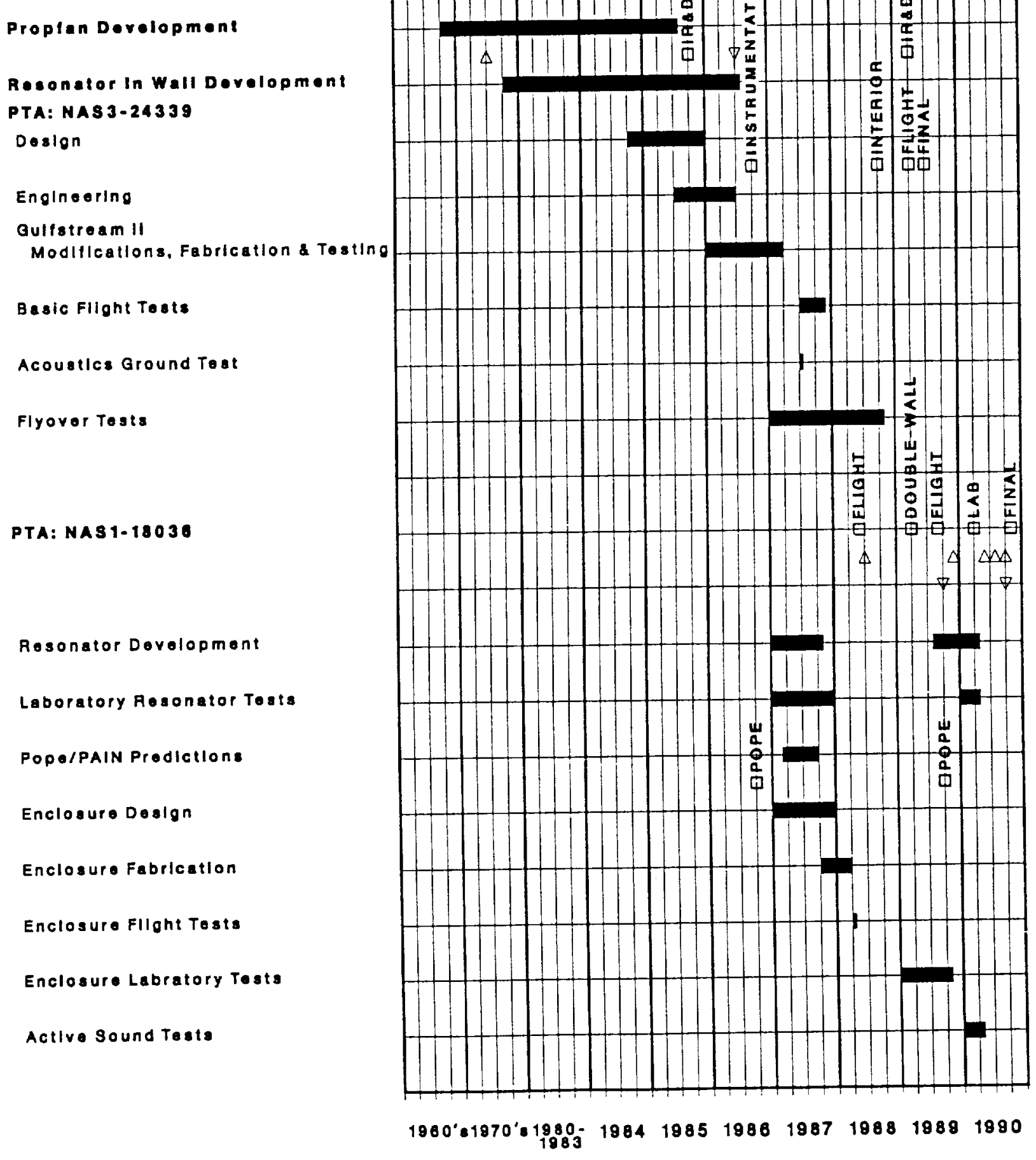

FIGURE 1: Project Timetable 


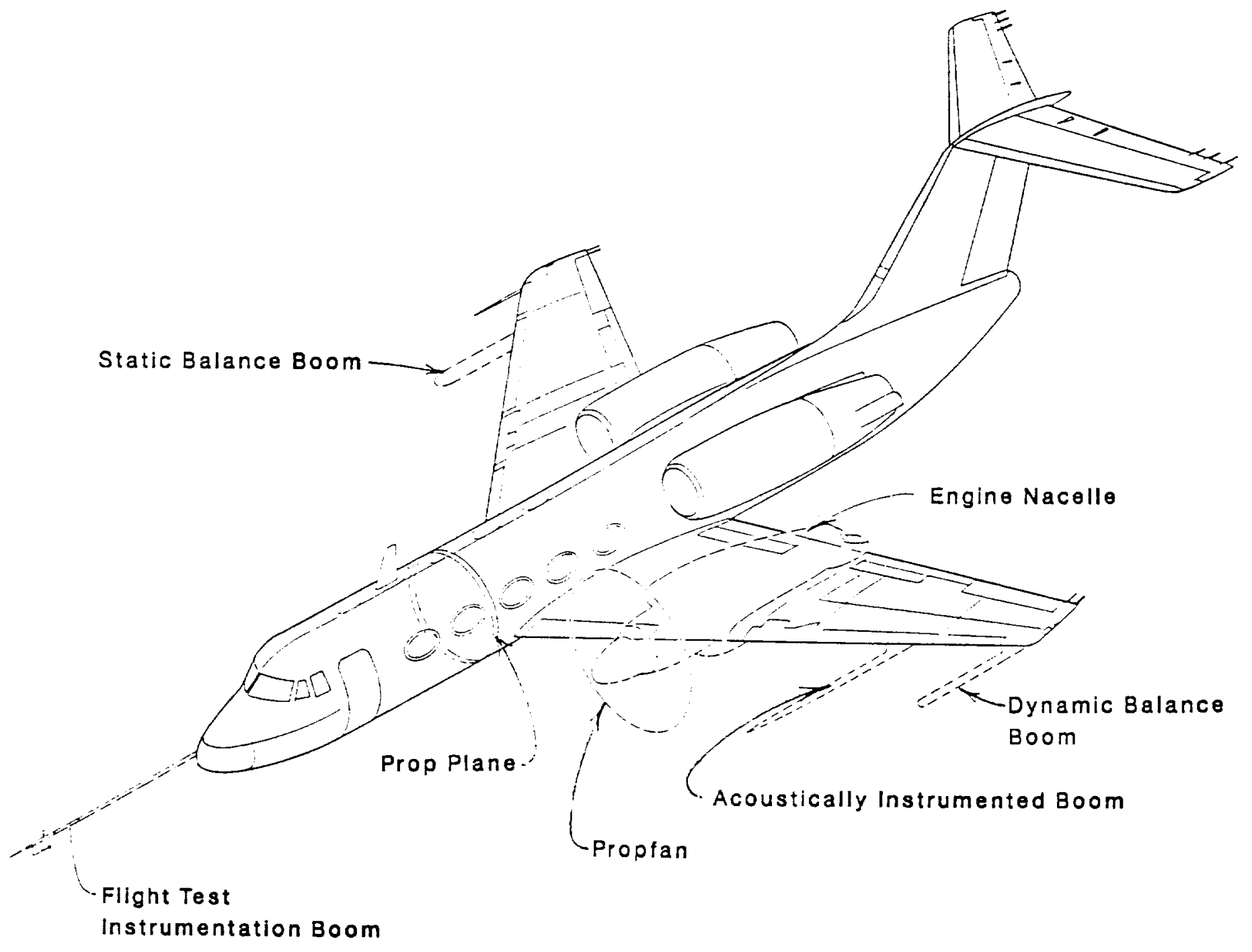

FIGURE 2: Sketch of PTA Alrcraft with WIng-Mounted Tractor Propfan. 

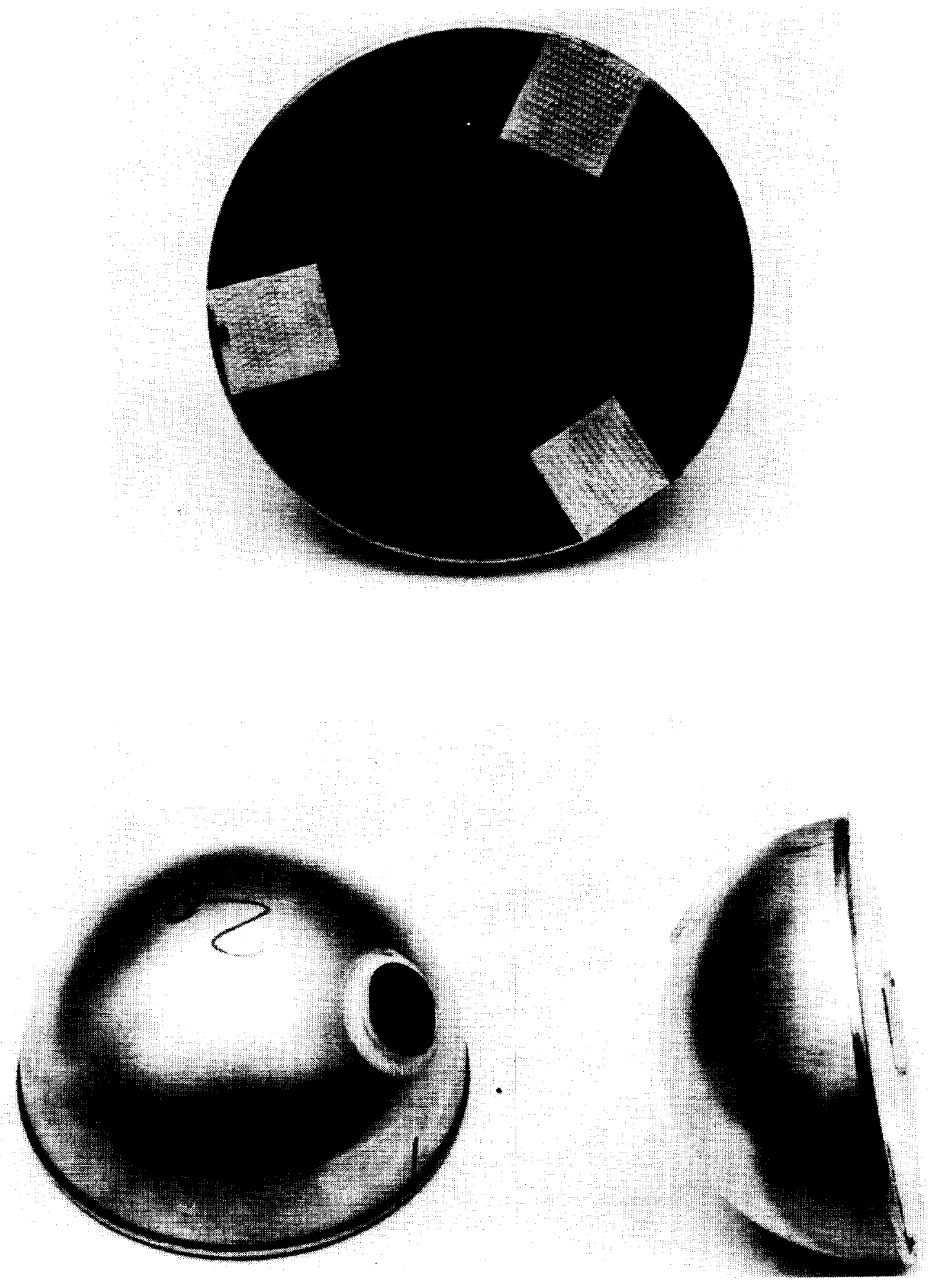

FIGURE 3: Flight Test Hemispherical Resonator Configuration. 


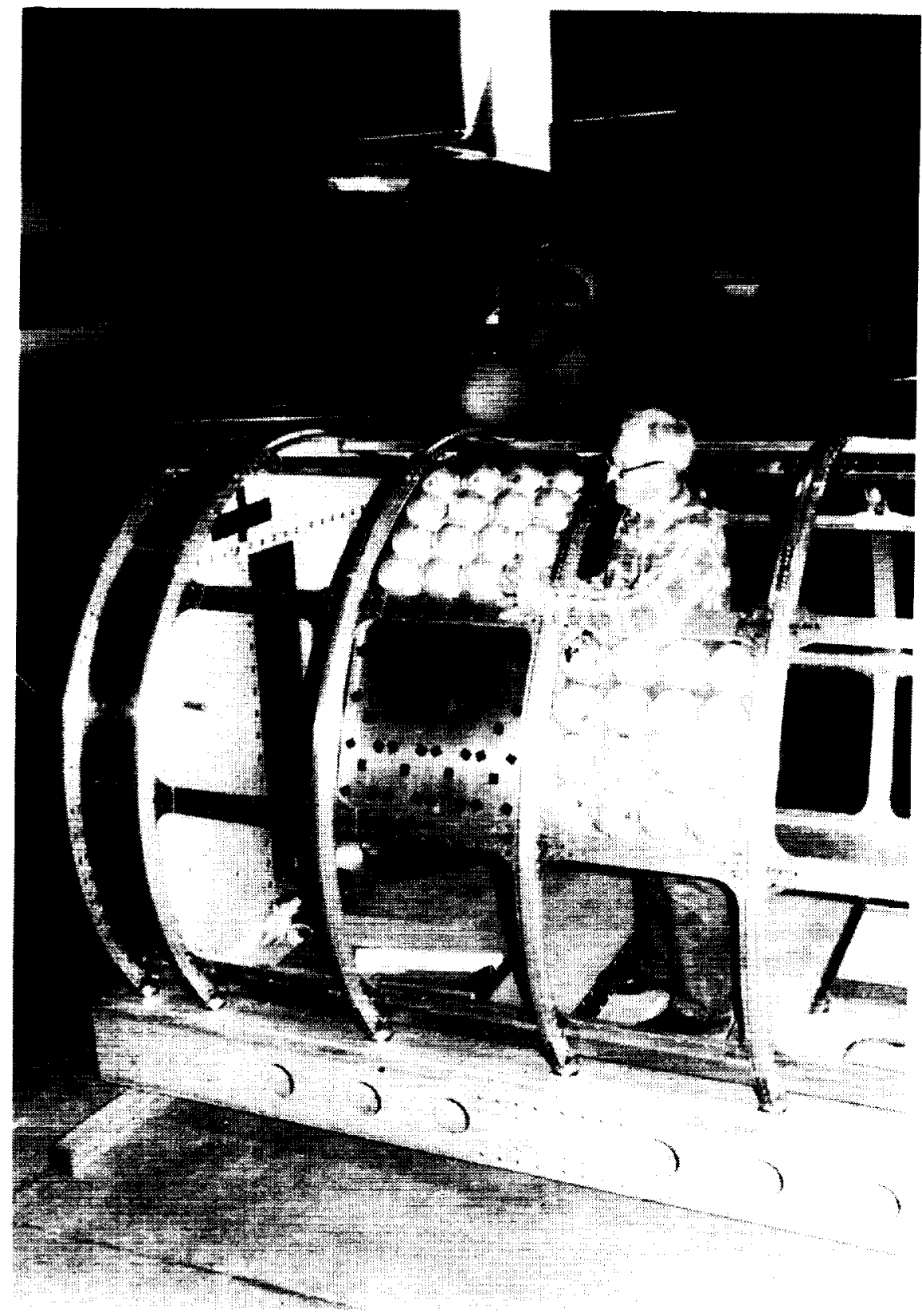

FIGURE 4: Photograph of Trim Panel Installation In Enclosure with Resonators Attached. 


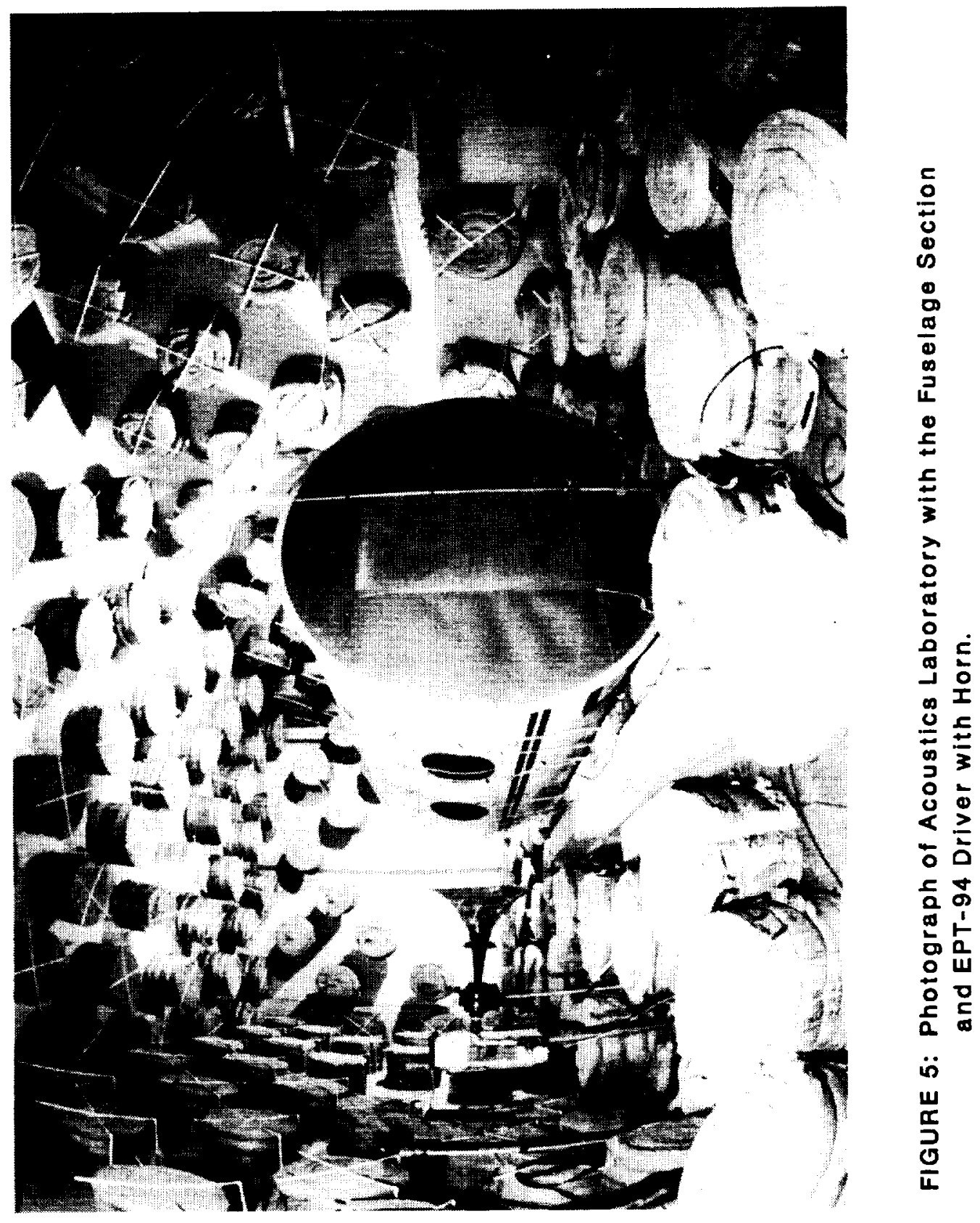




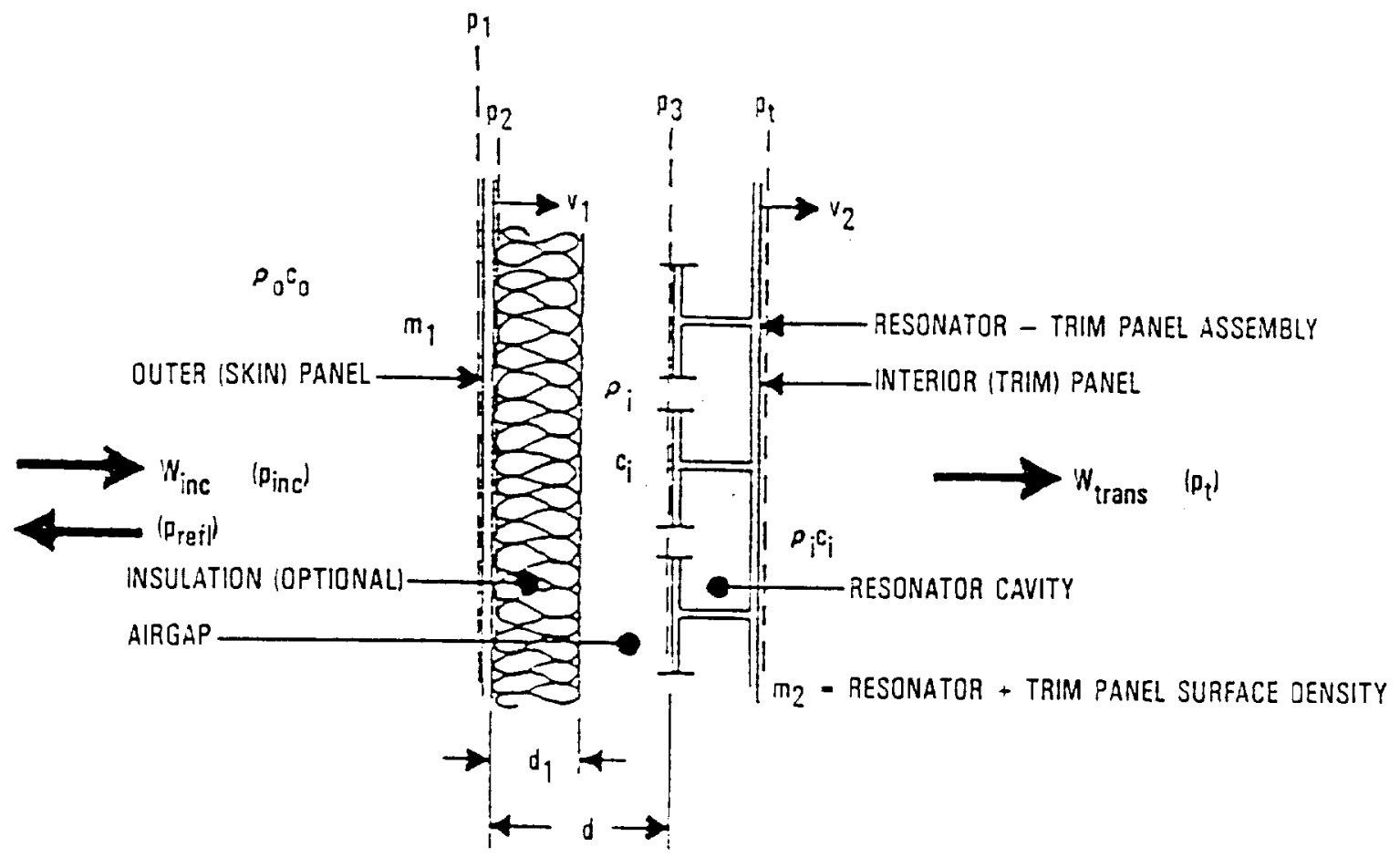

MODULE AREA, S - MA

M RESONATORSIMODULE

$\sigma=A_{t} \mid A$

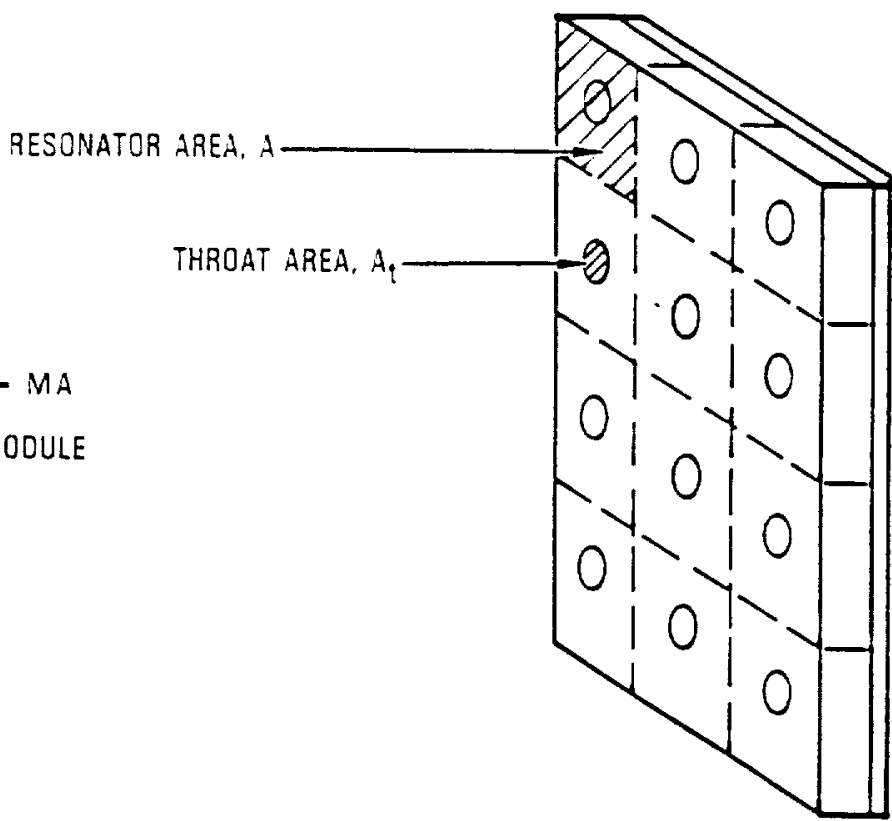

FIGURE 6: Flat Double-Panel Wall Assembly with Resonators. 


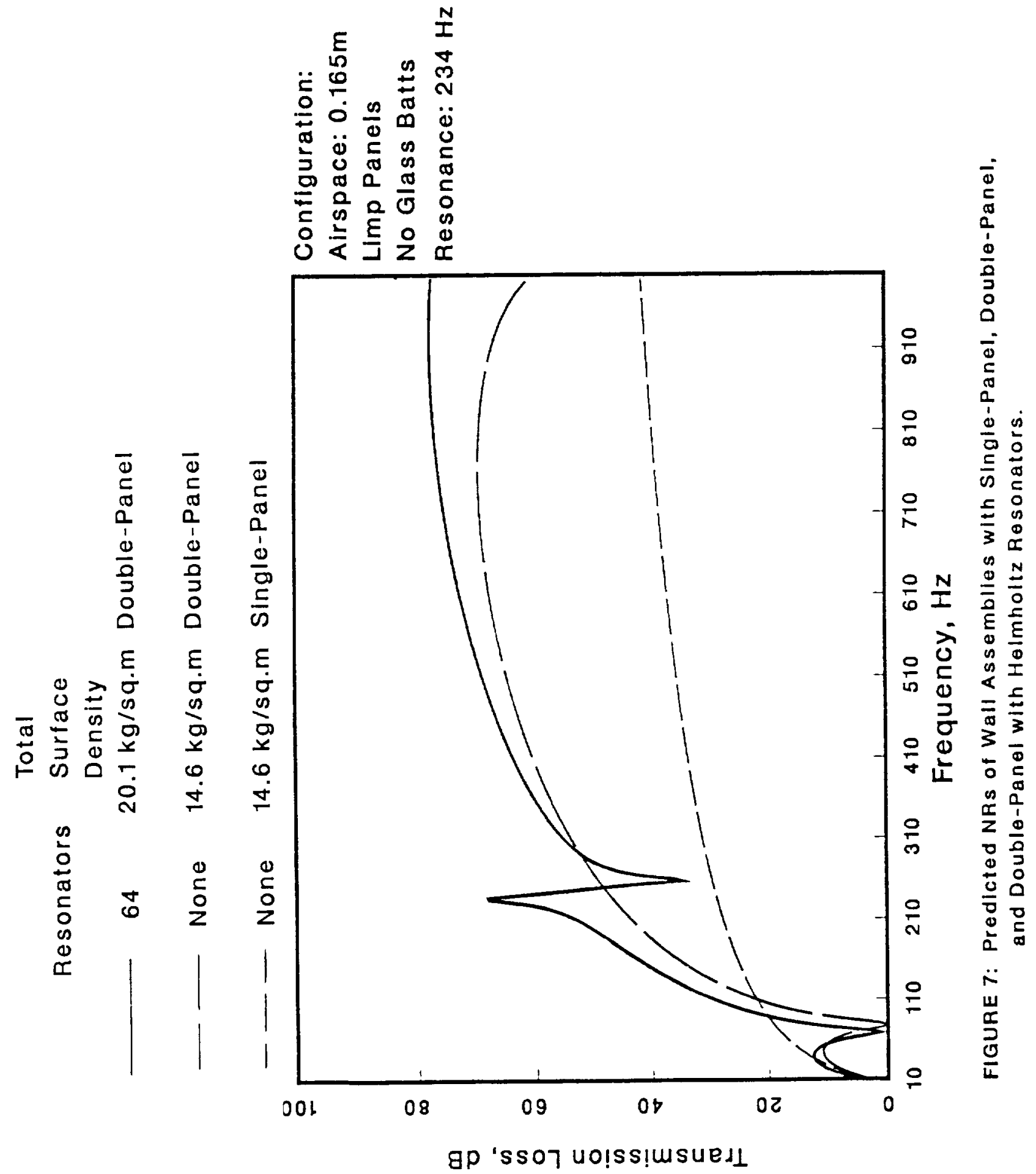




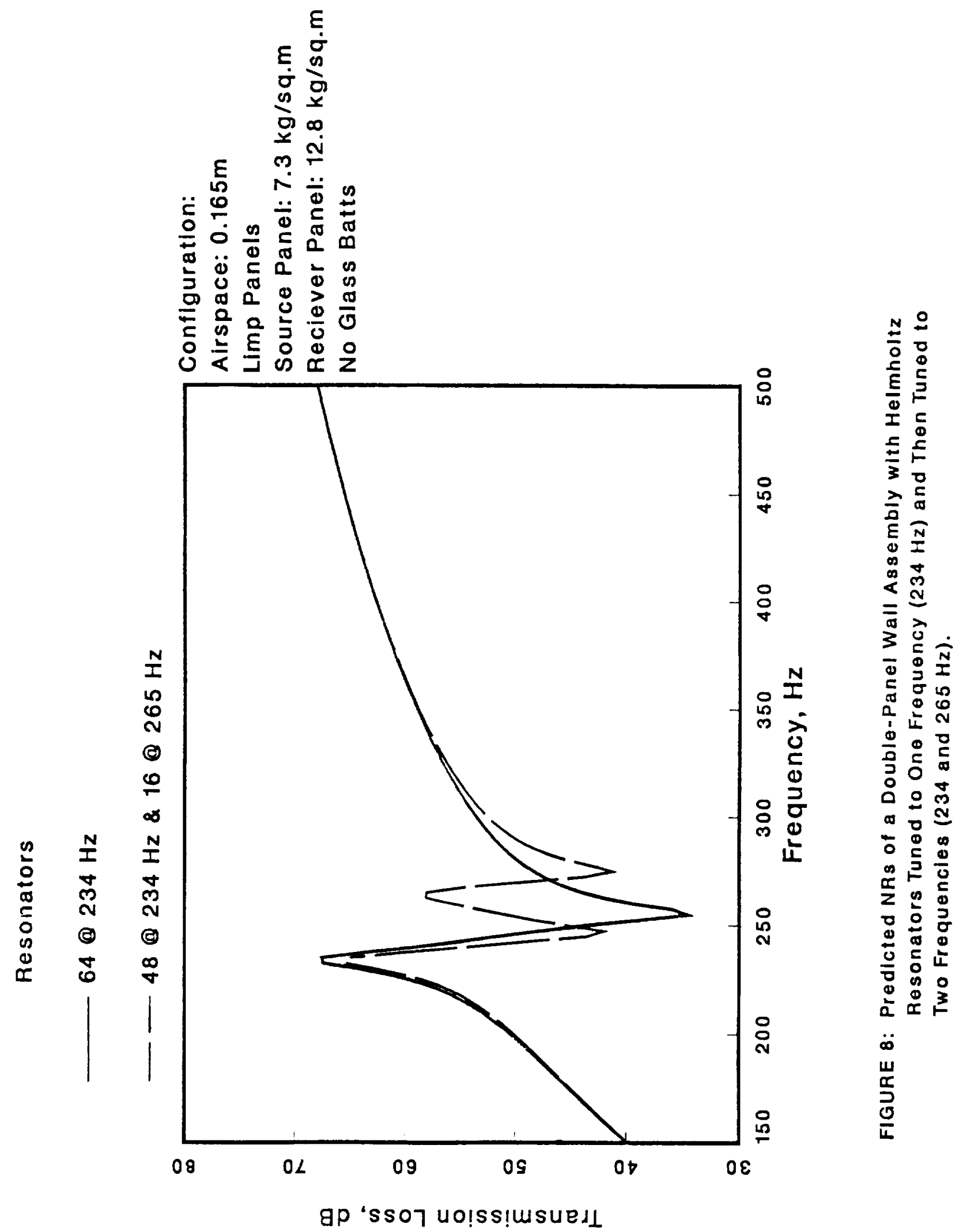




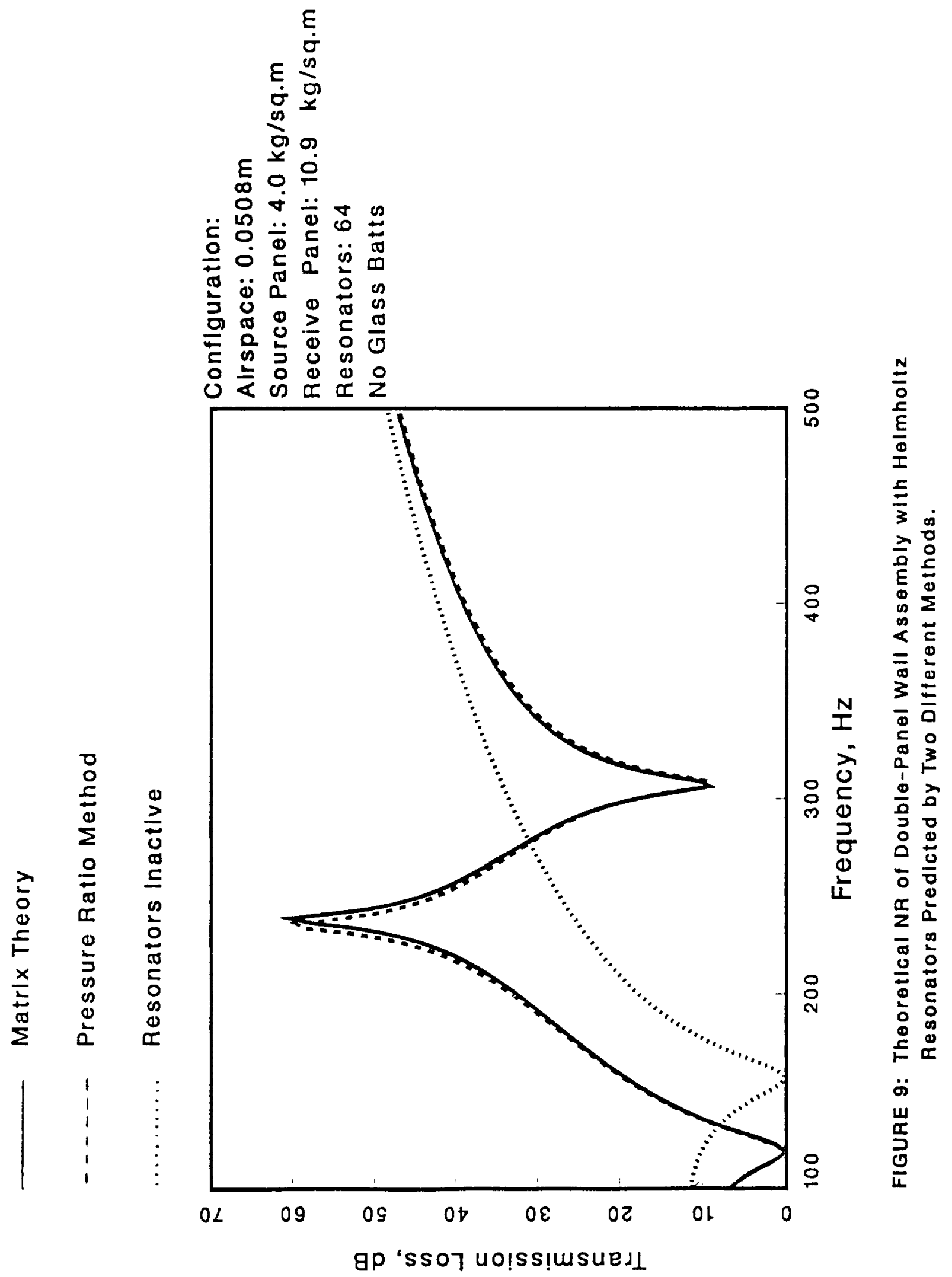




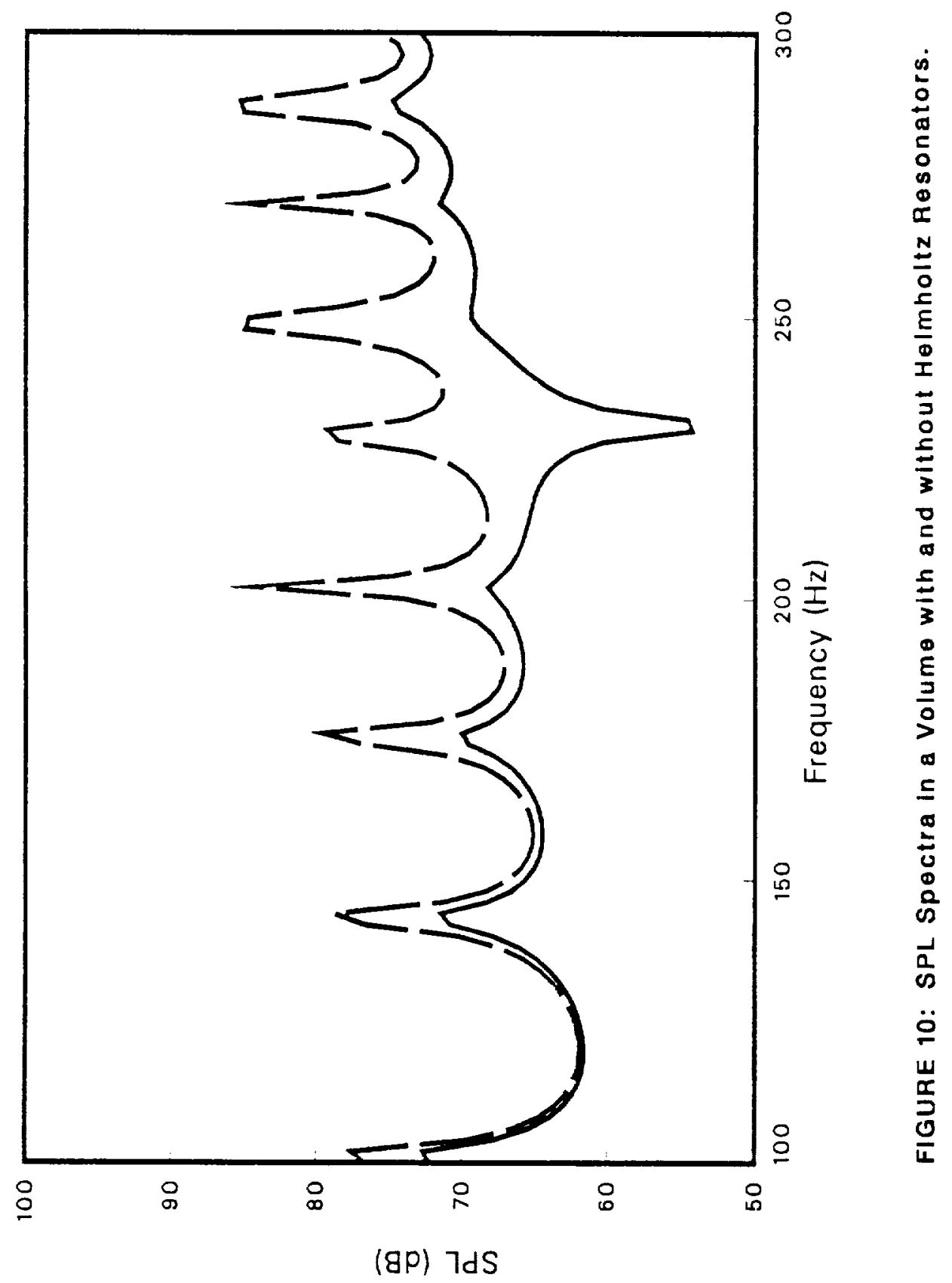



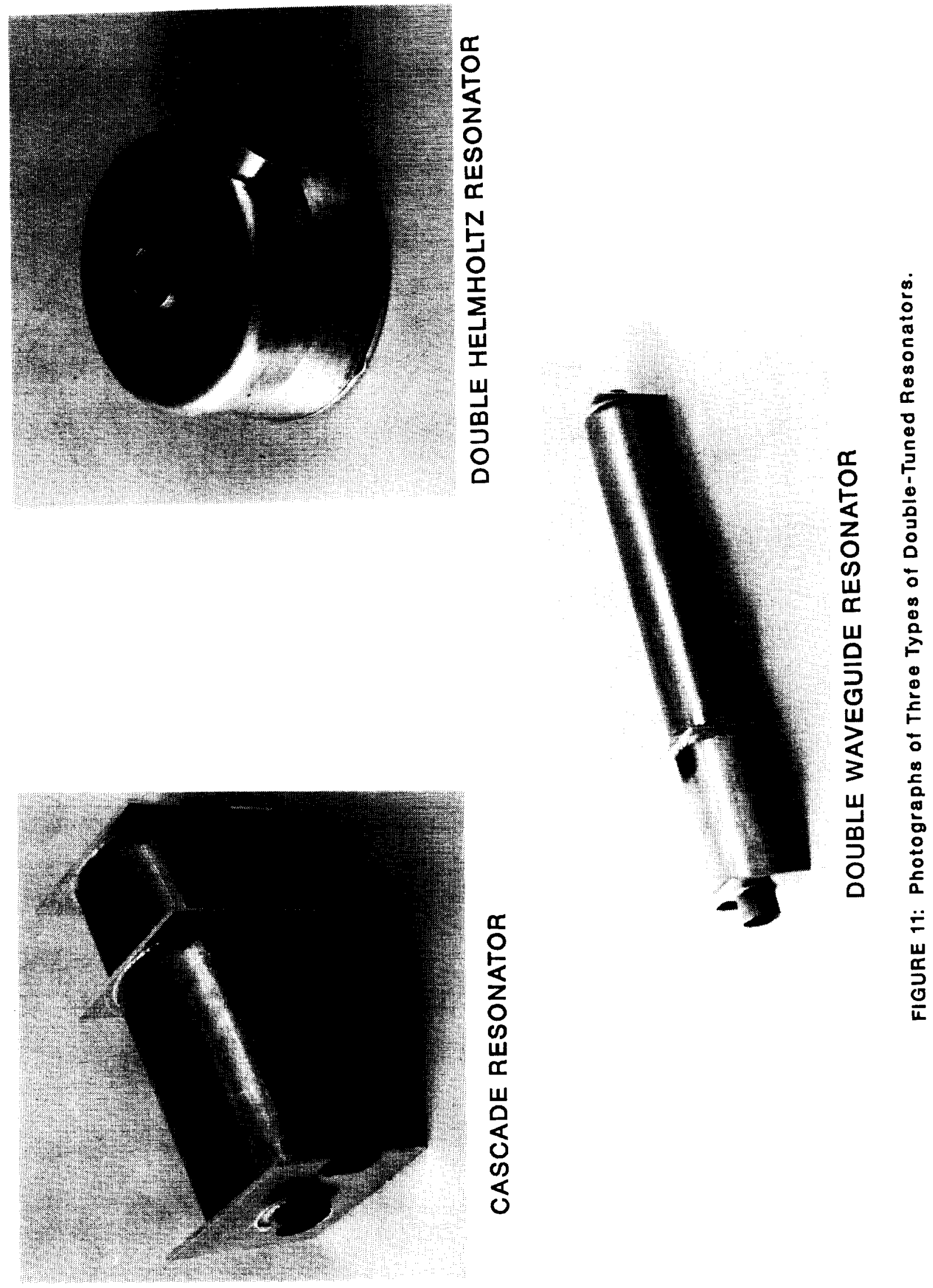

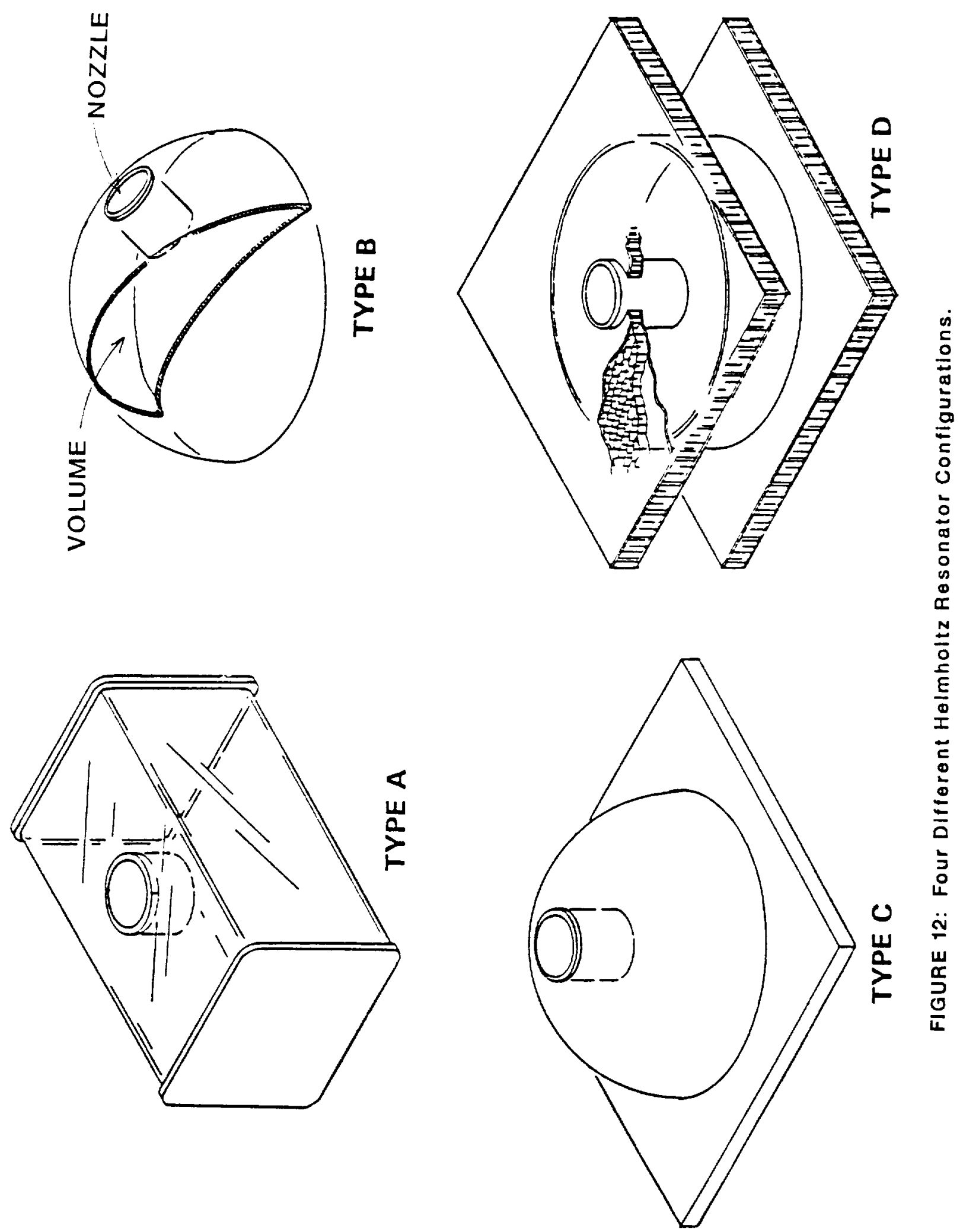


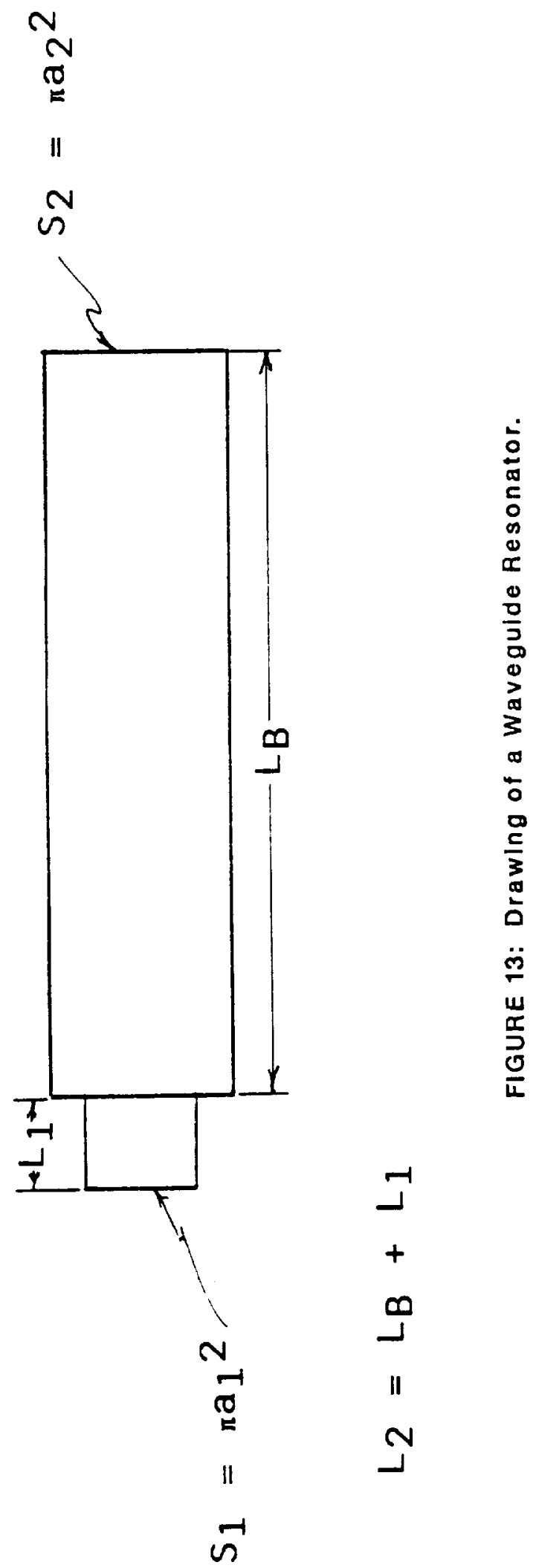




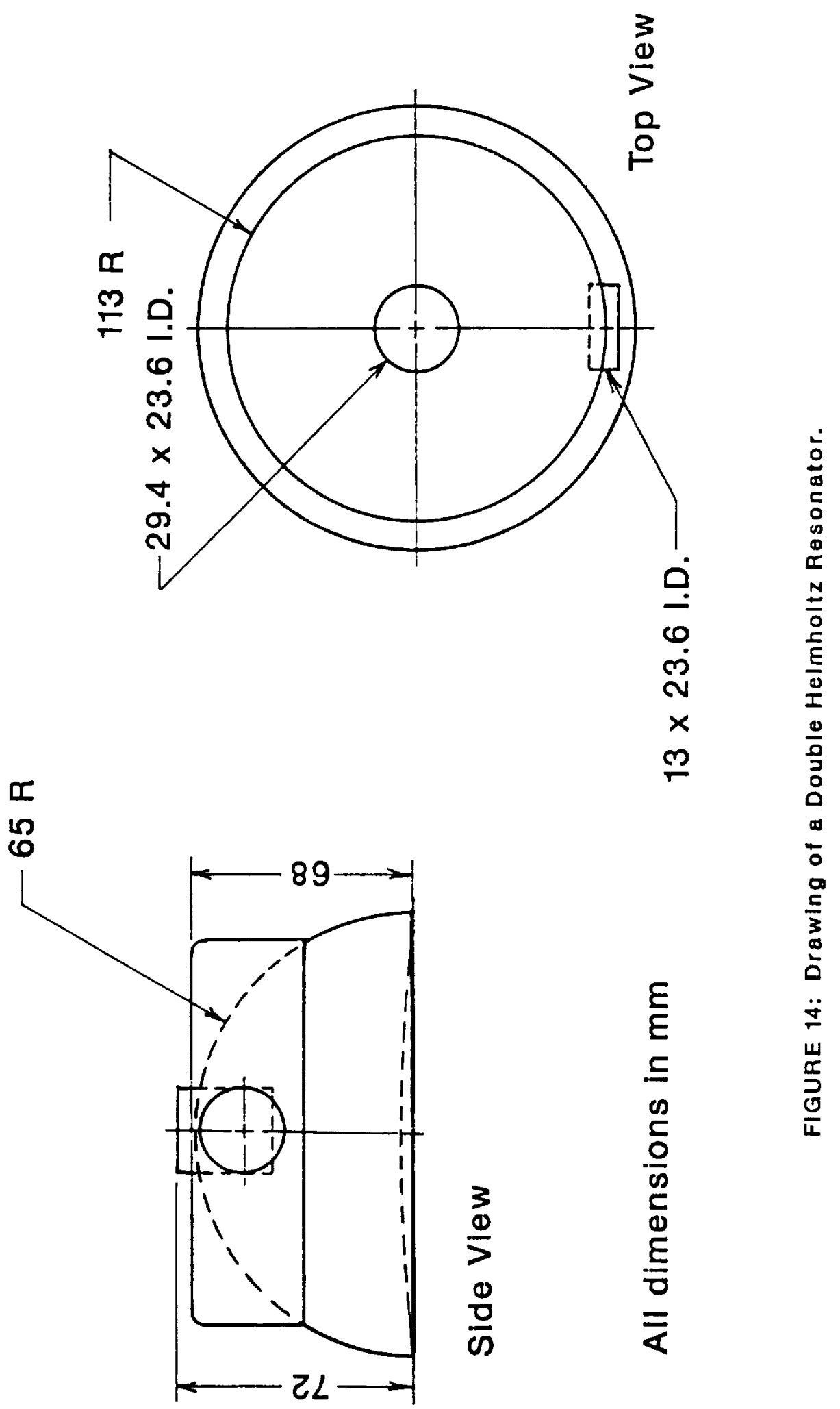




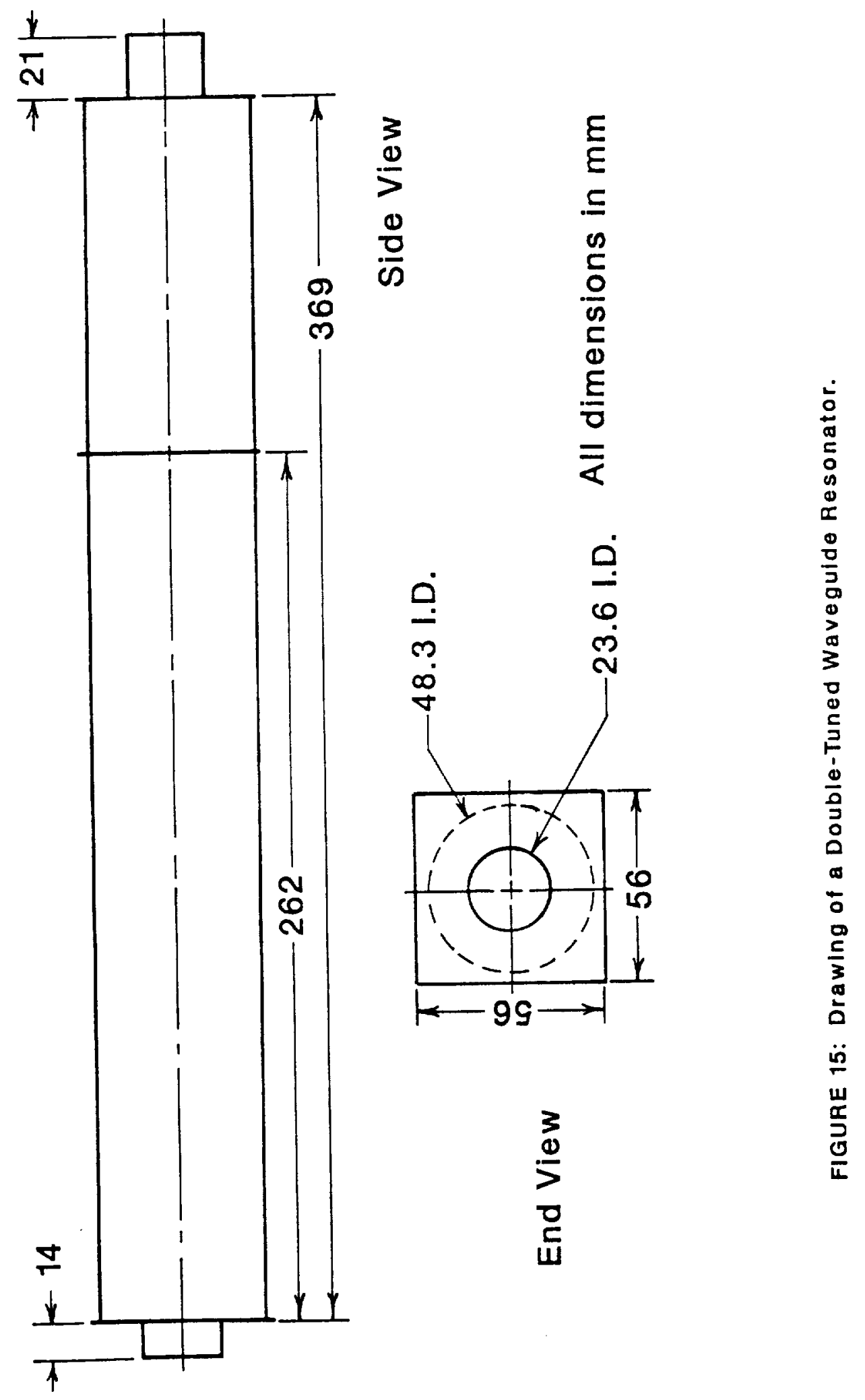



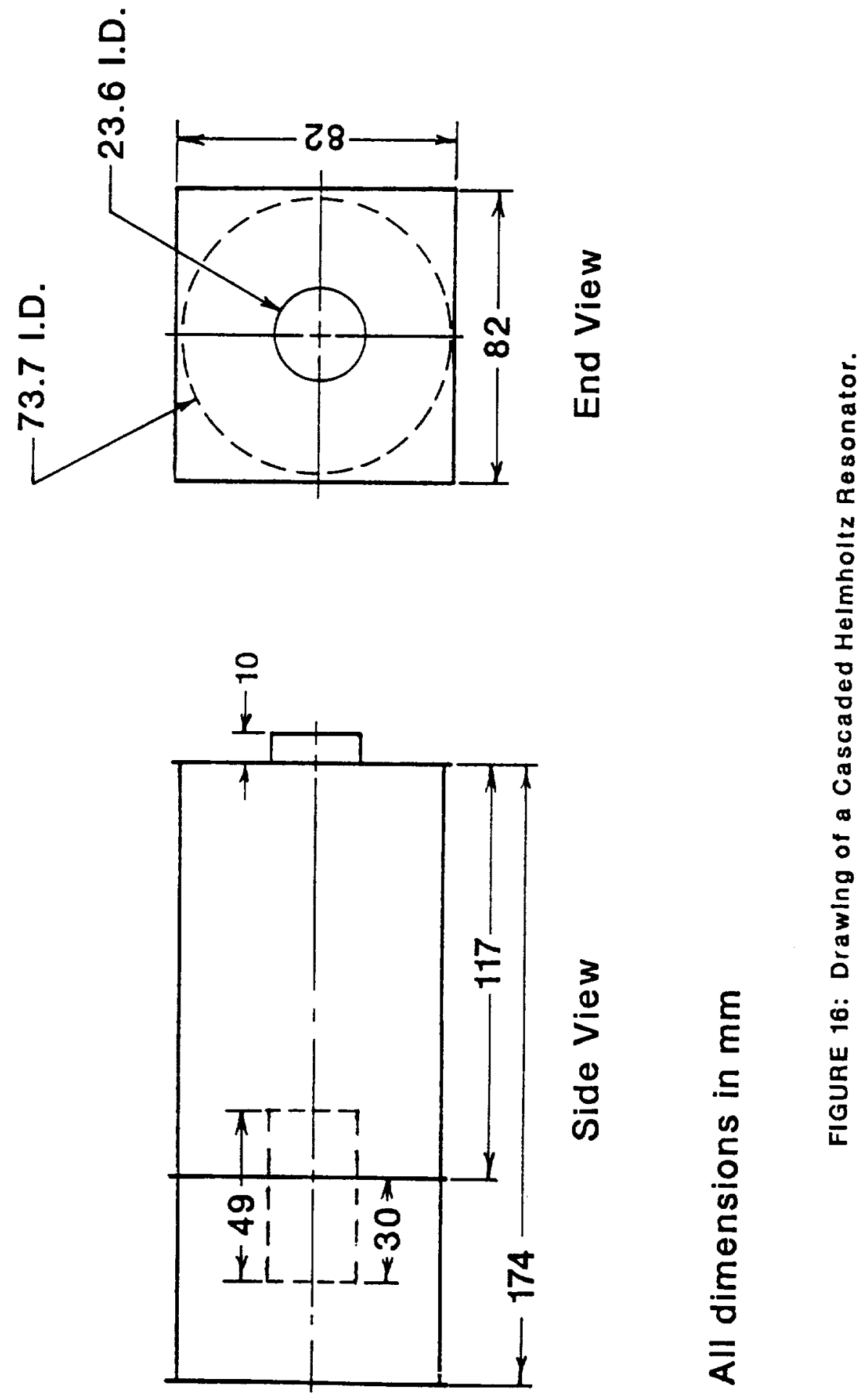


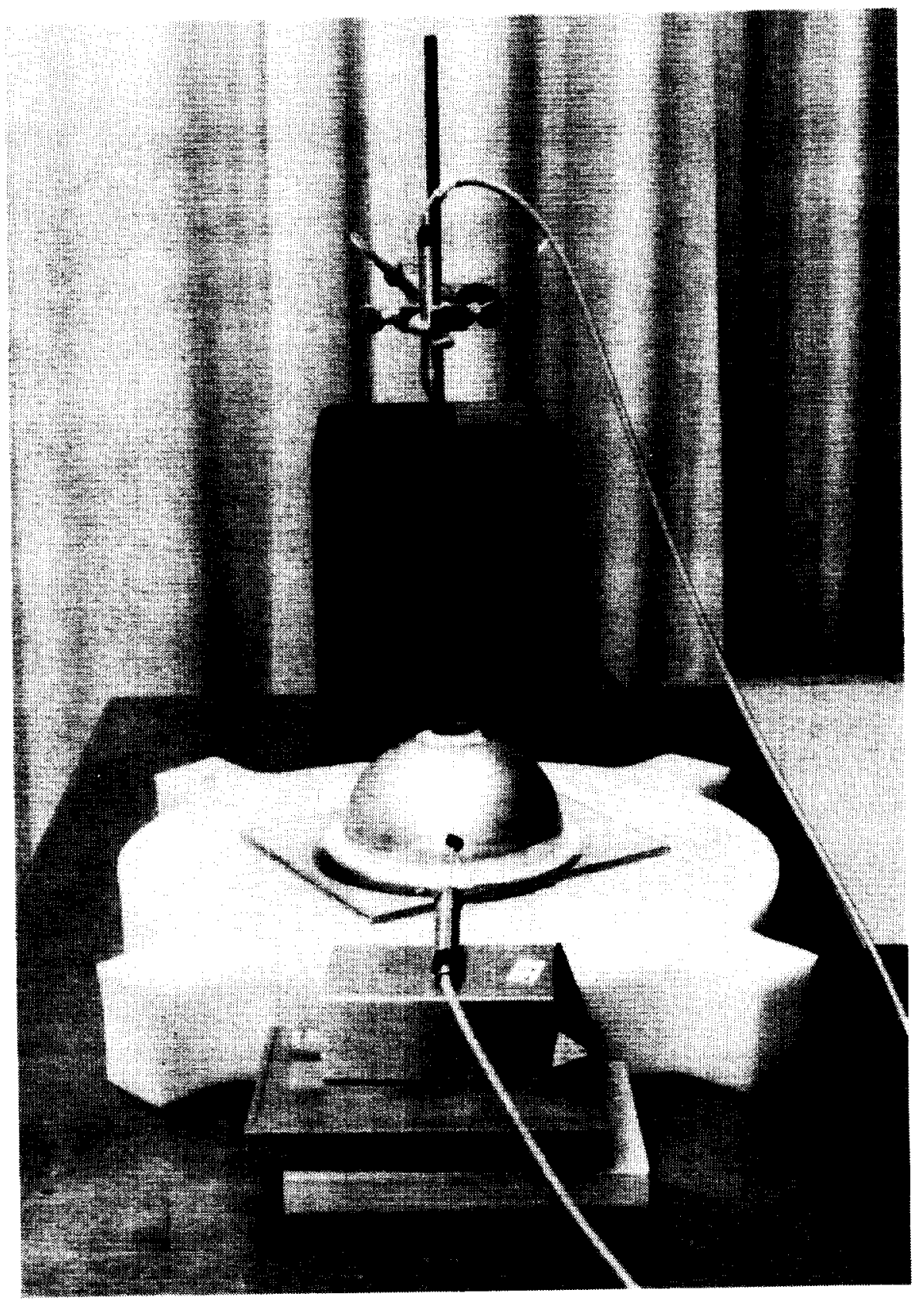

FIGURE 17: Test Setup - Evaluation of Individual Resonators. 


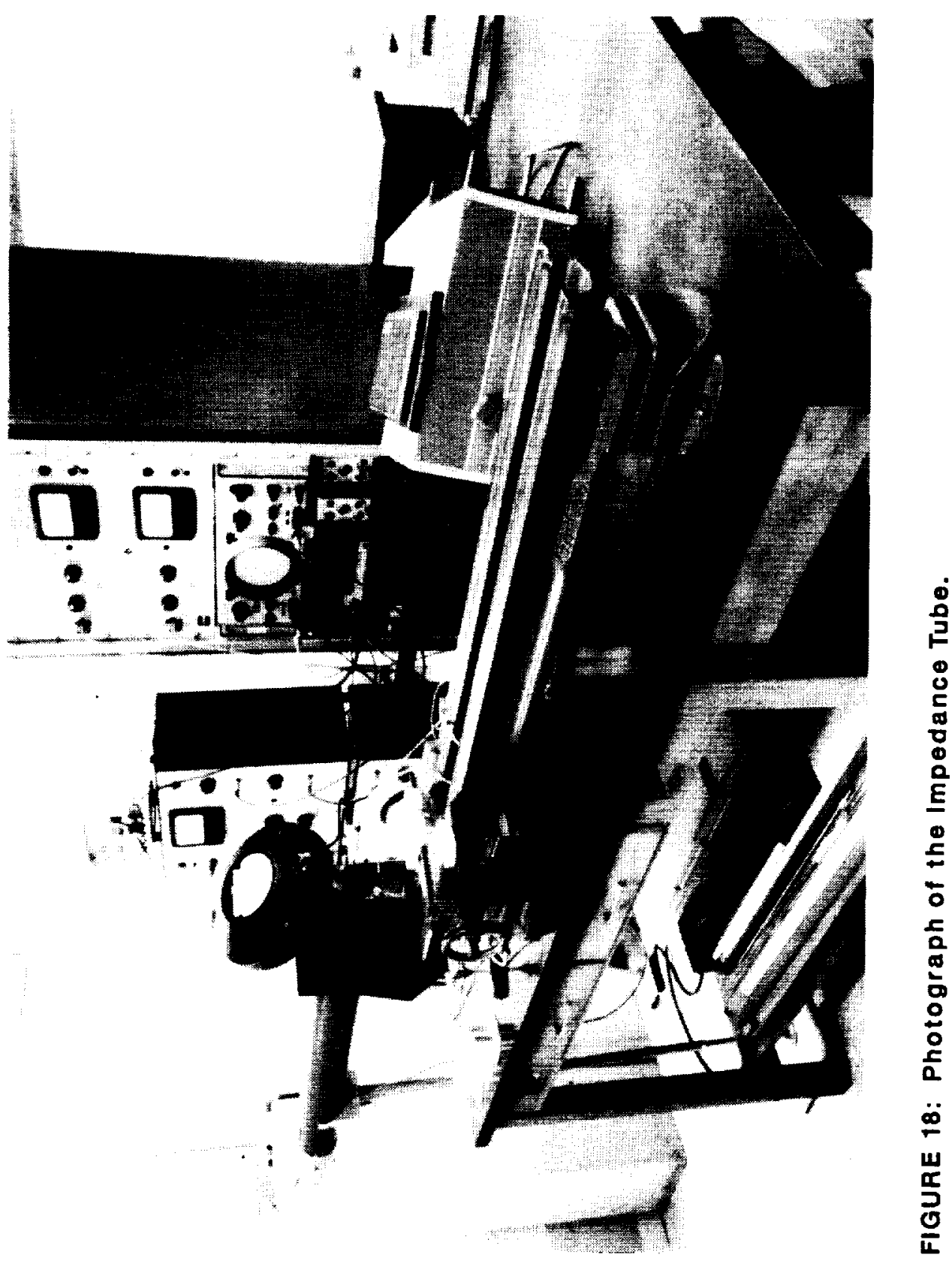




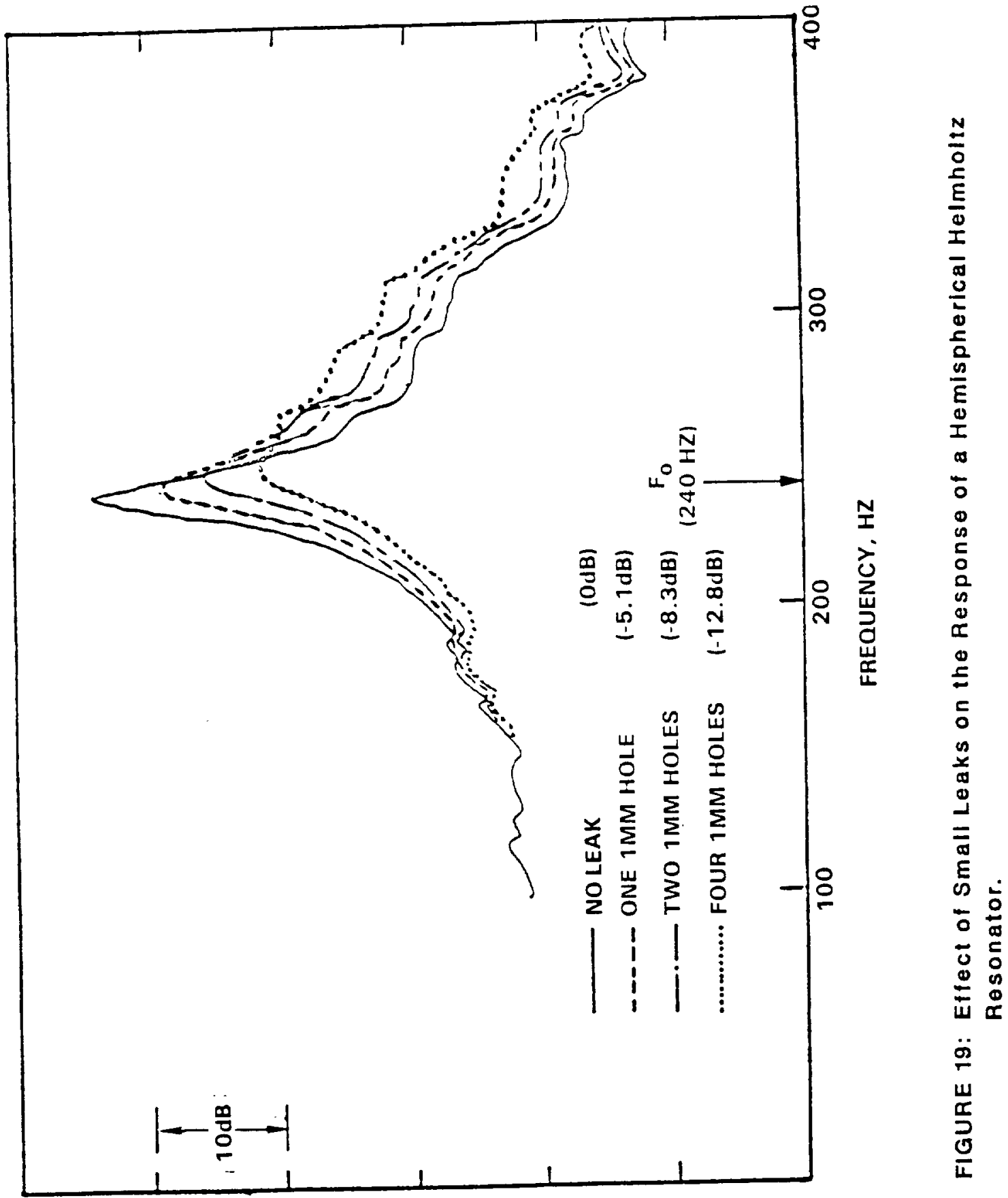

(ap) NOIL 


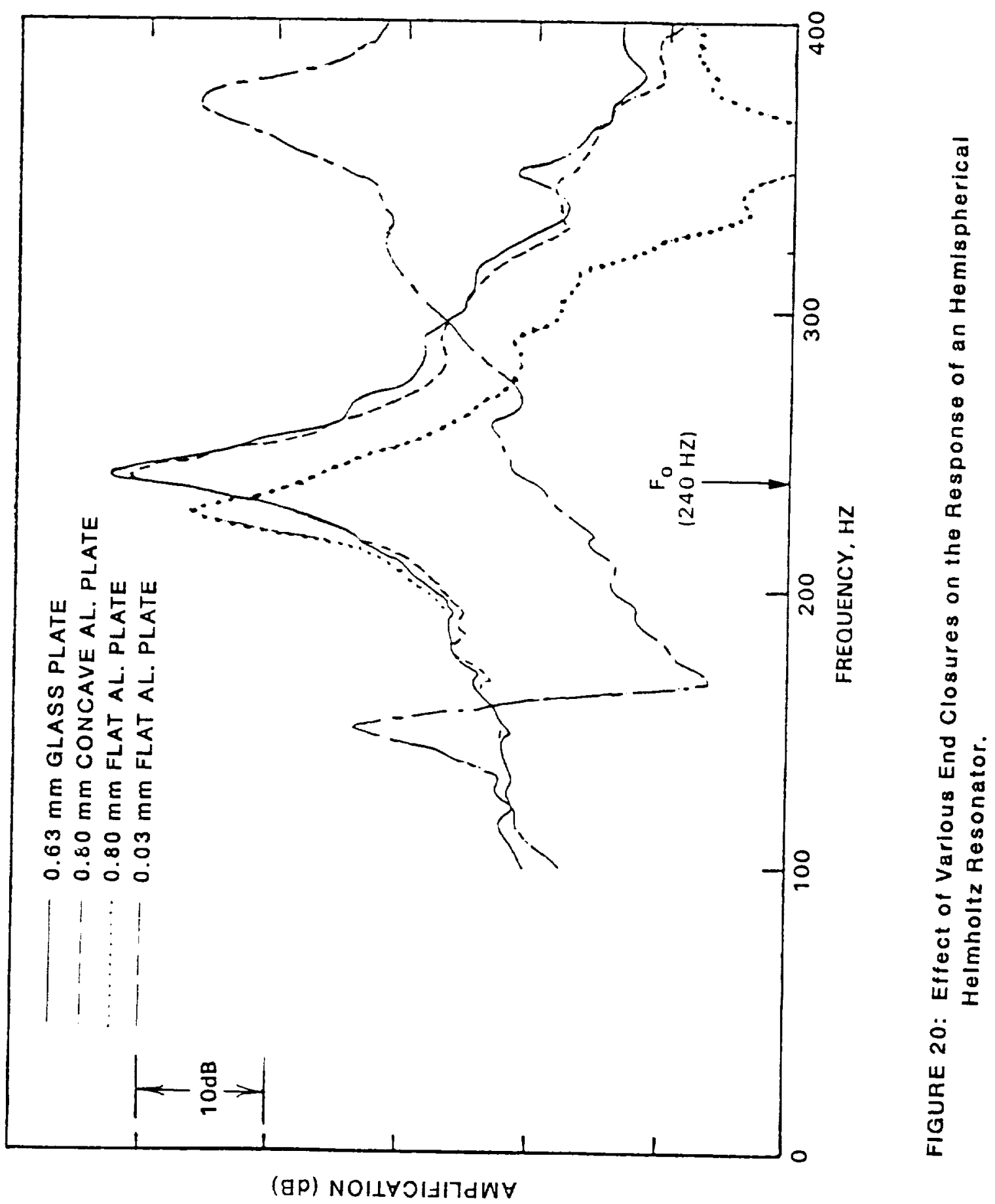




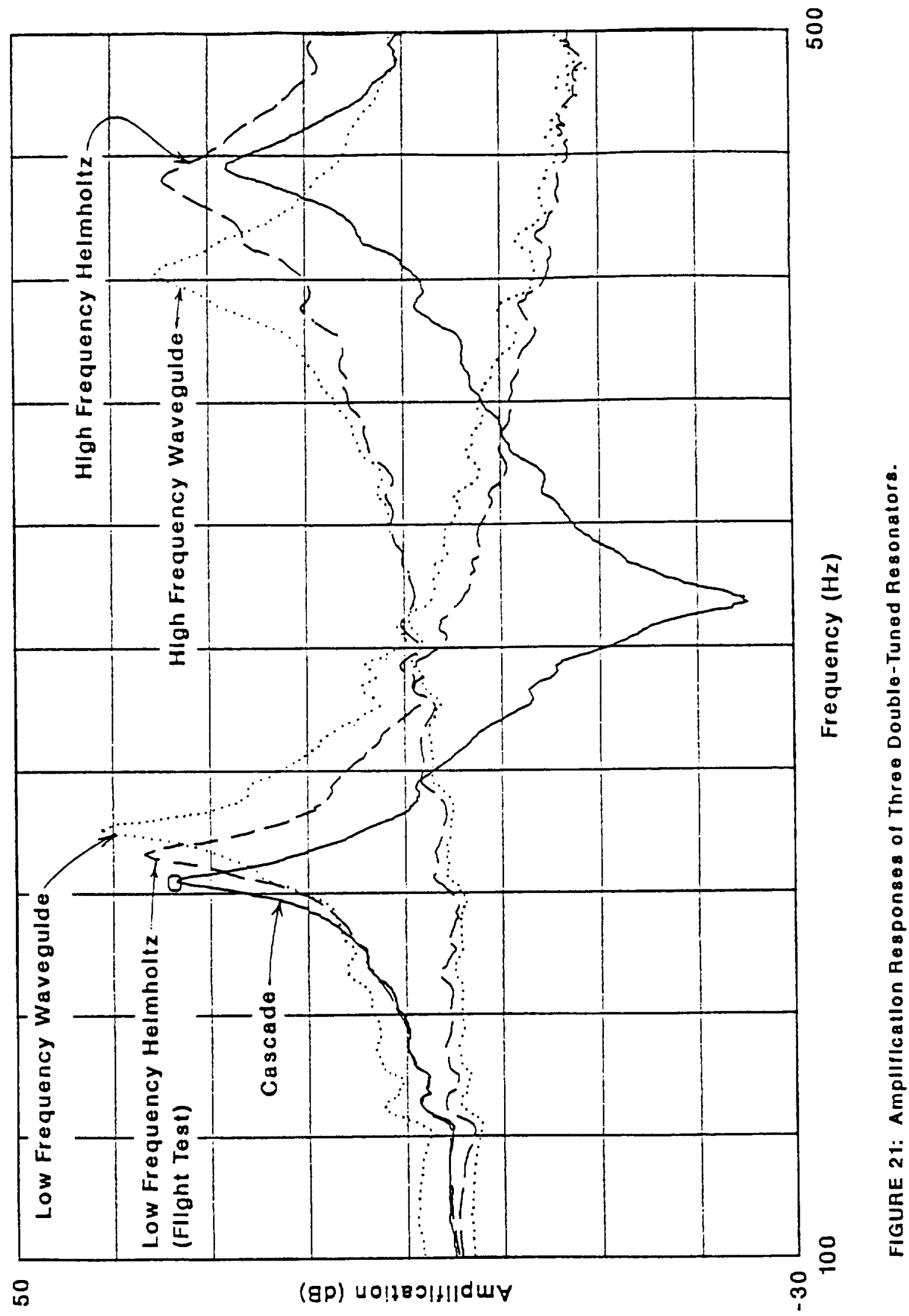




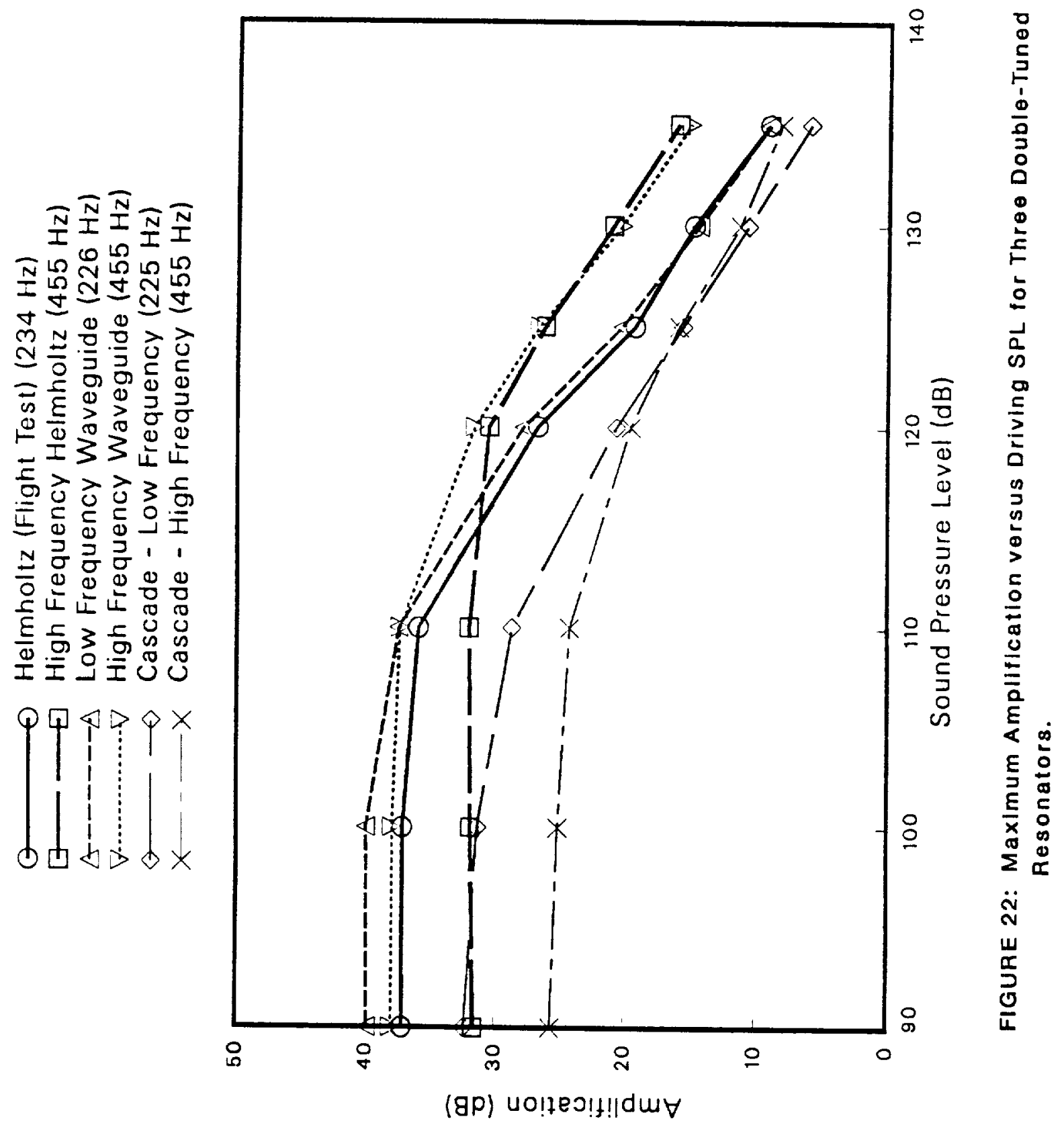




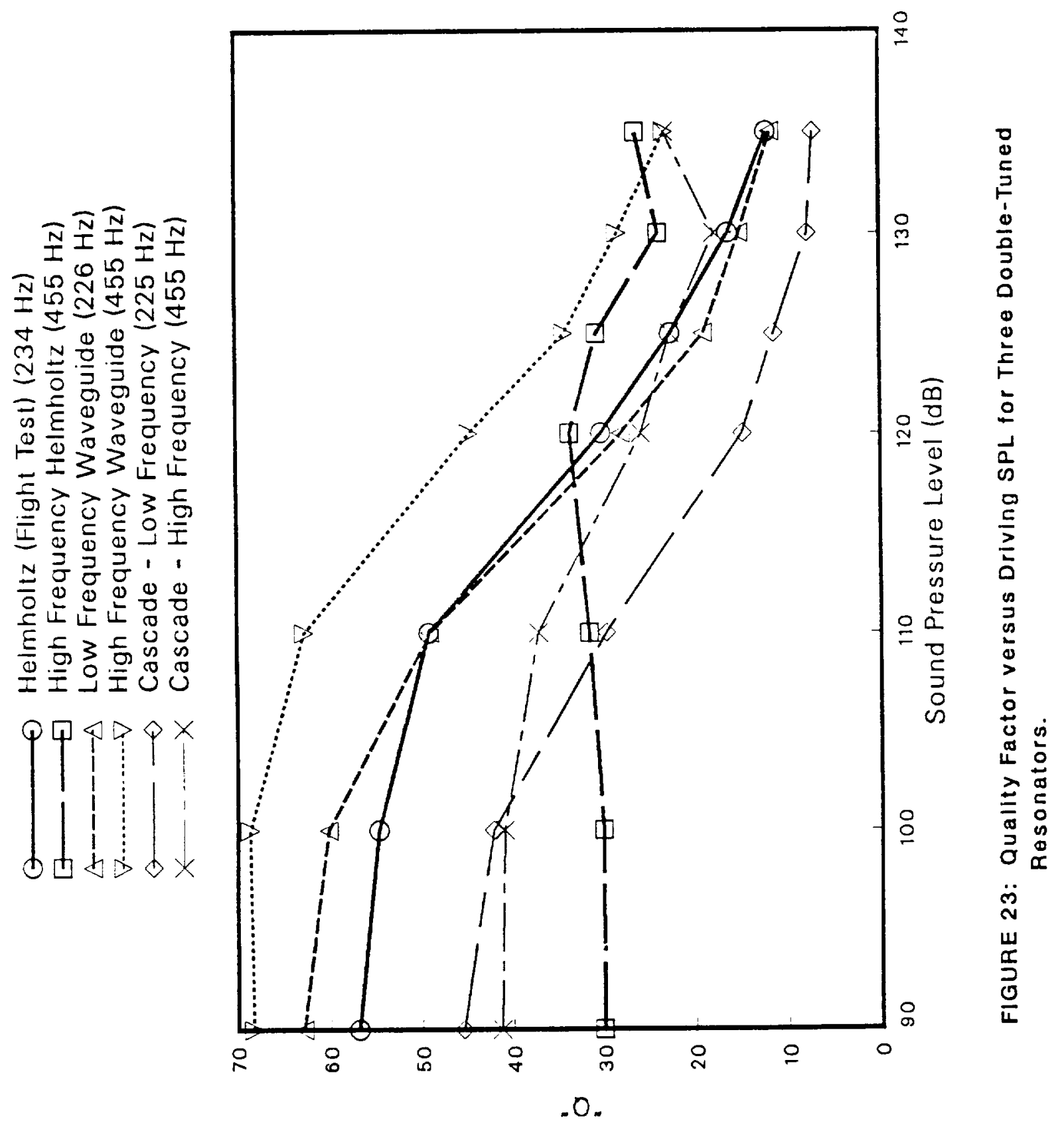




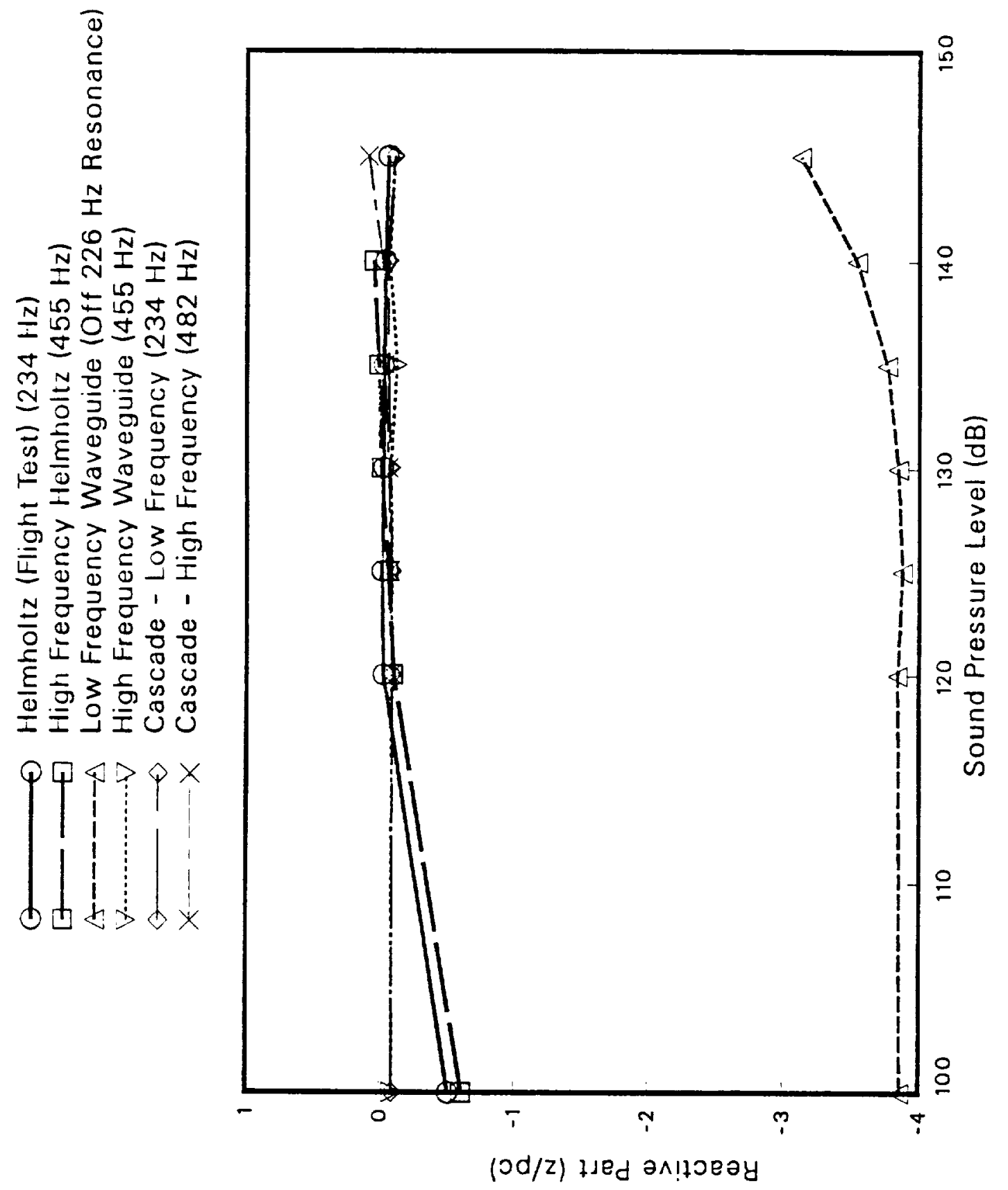

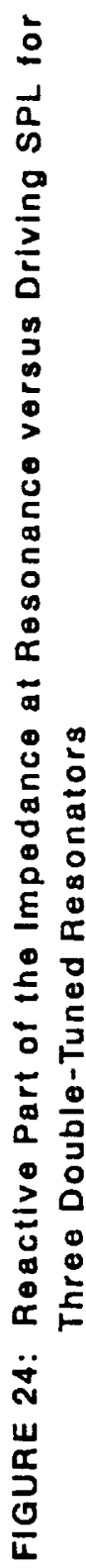



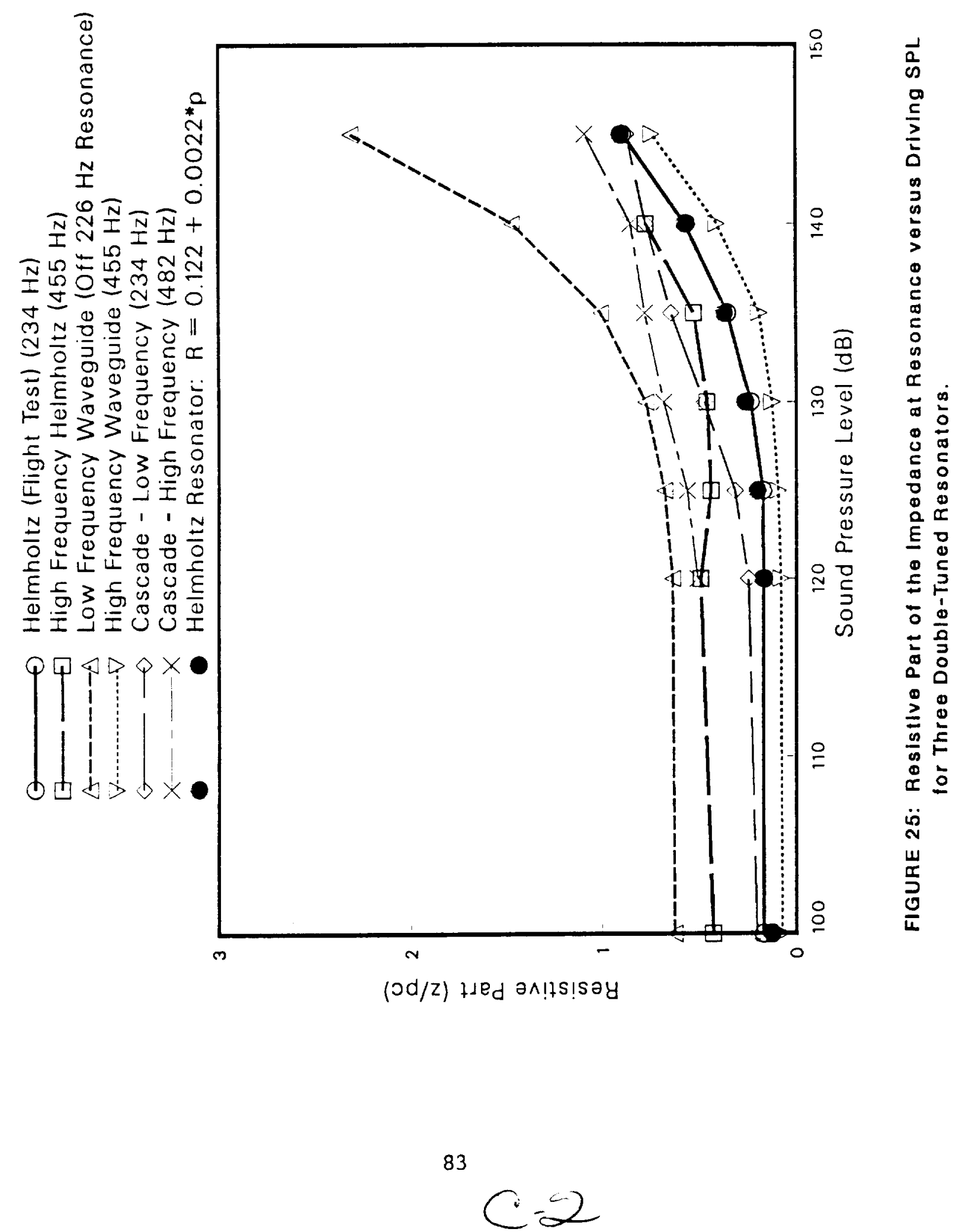


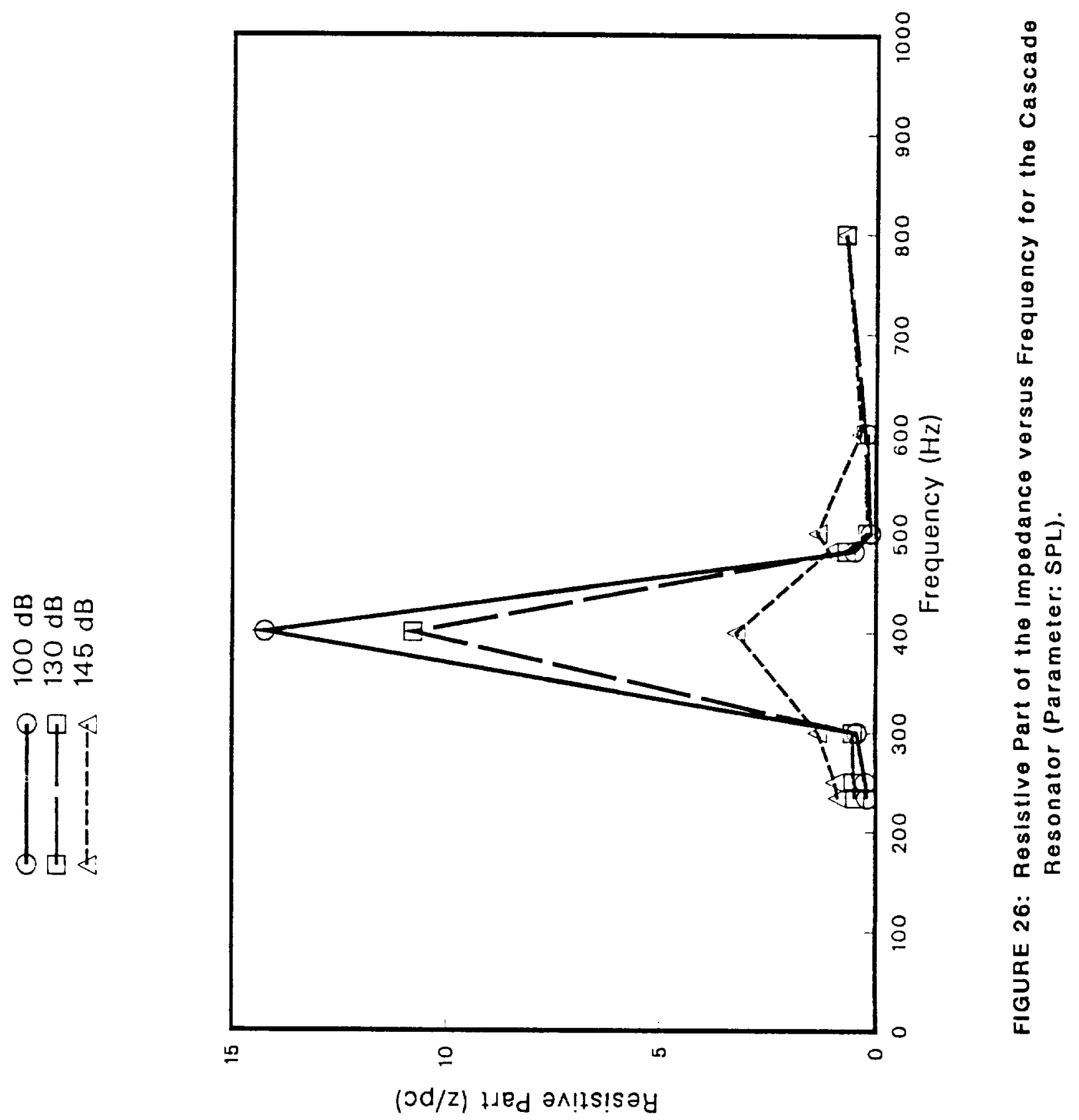




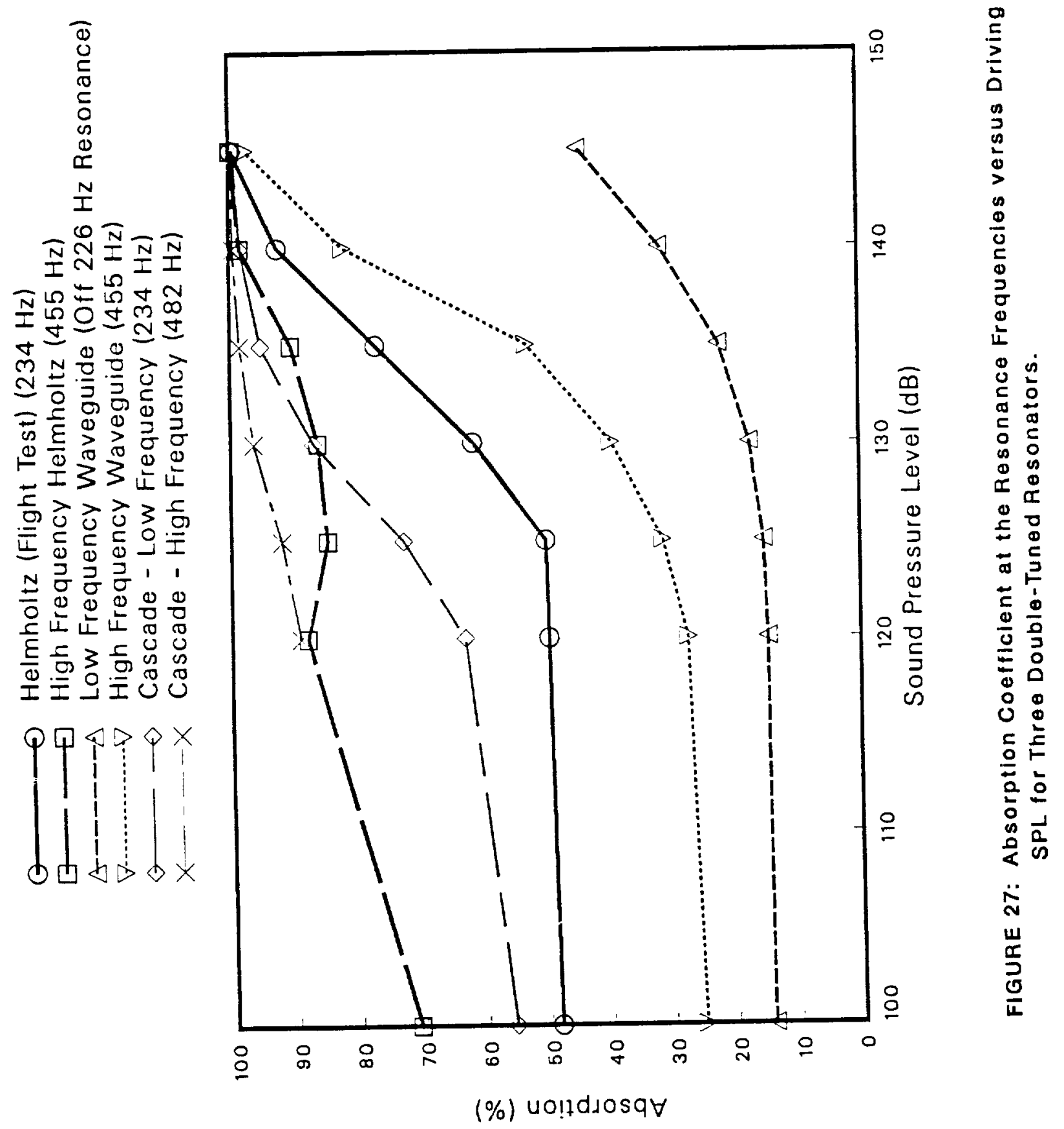




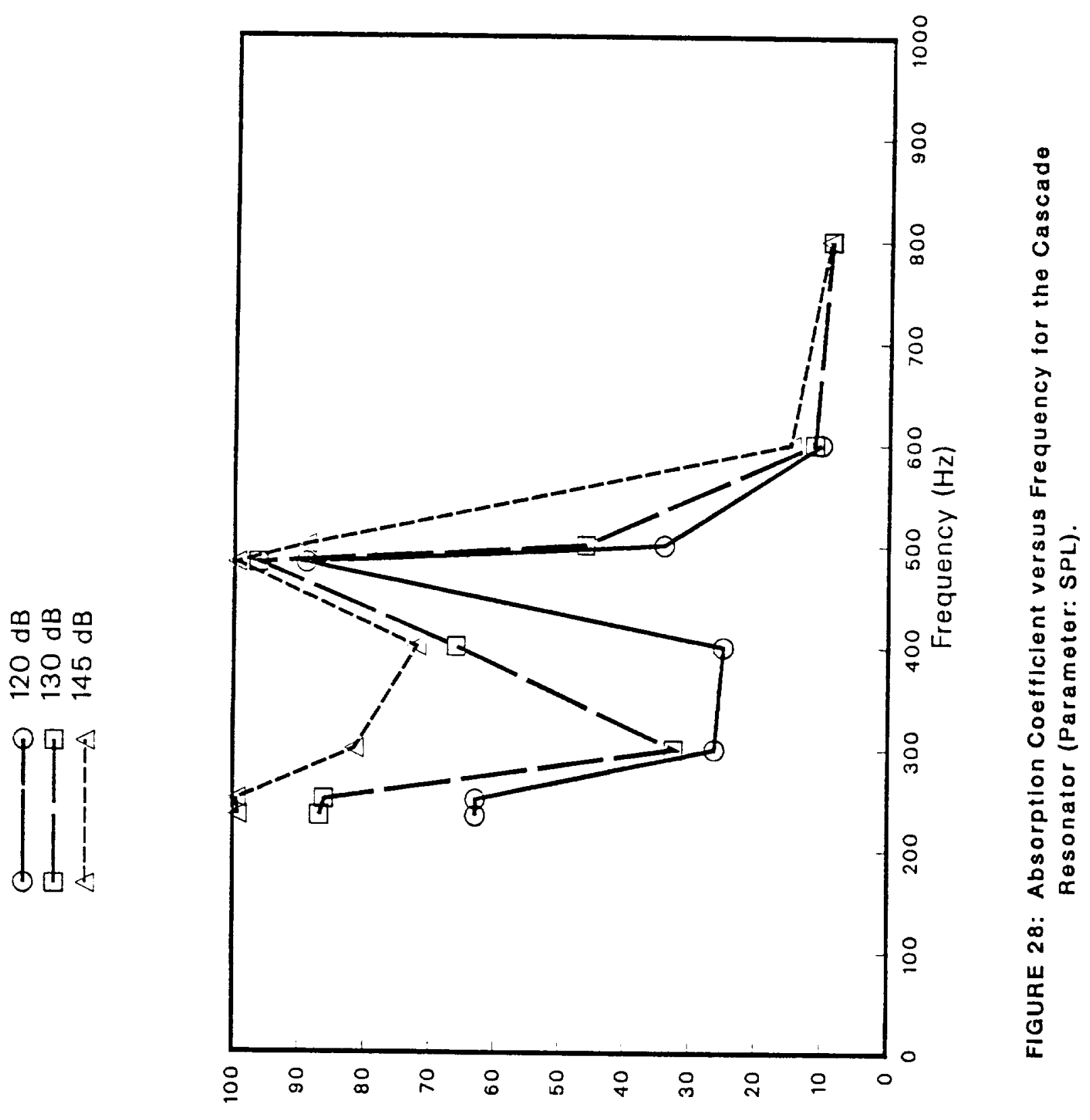

(\%) vo!tdıosq 


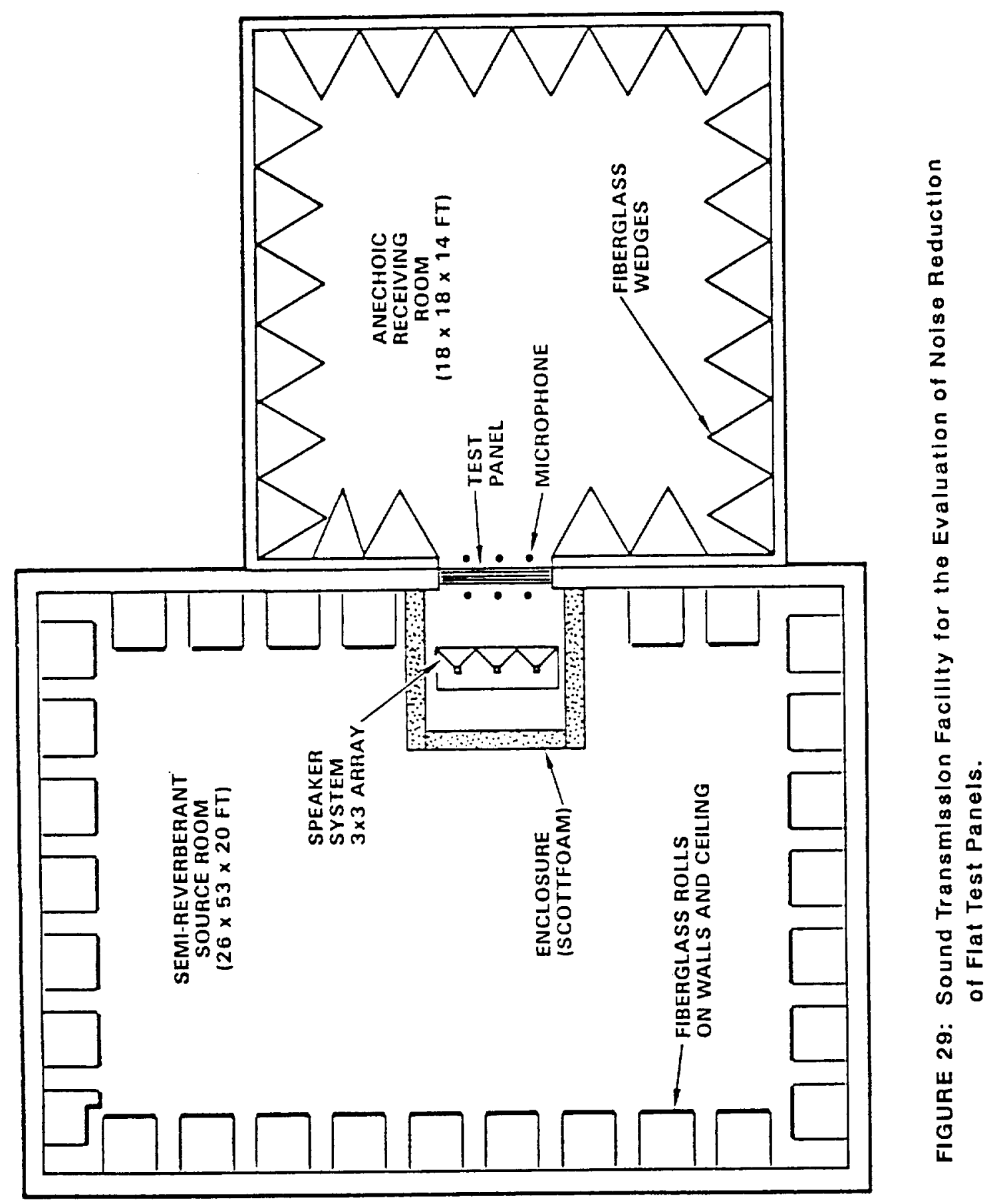




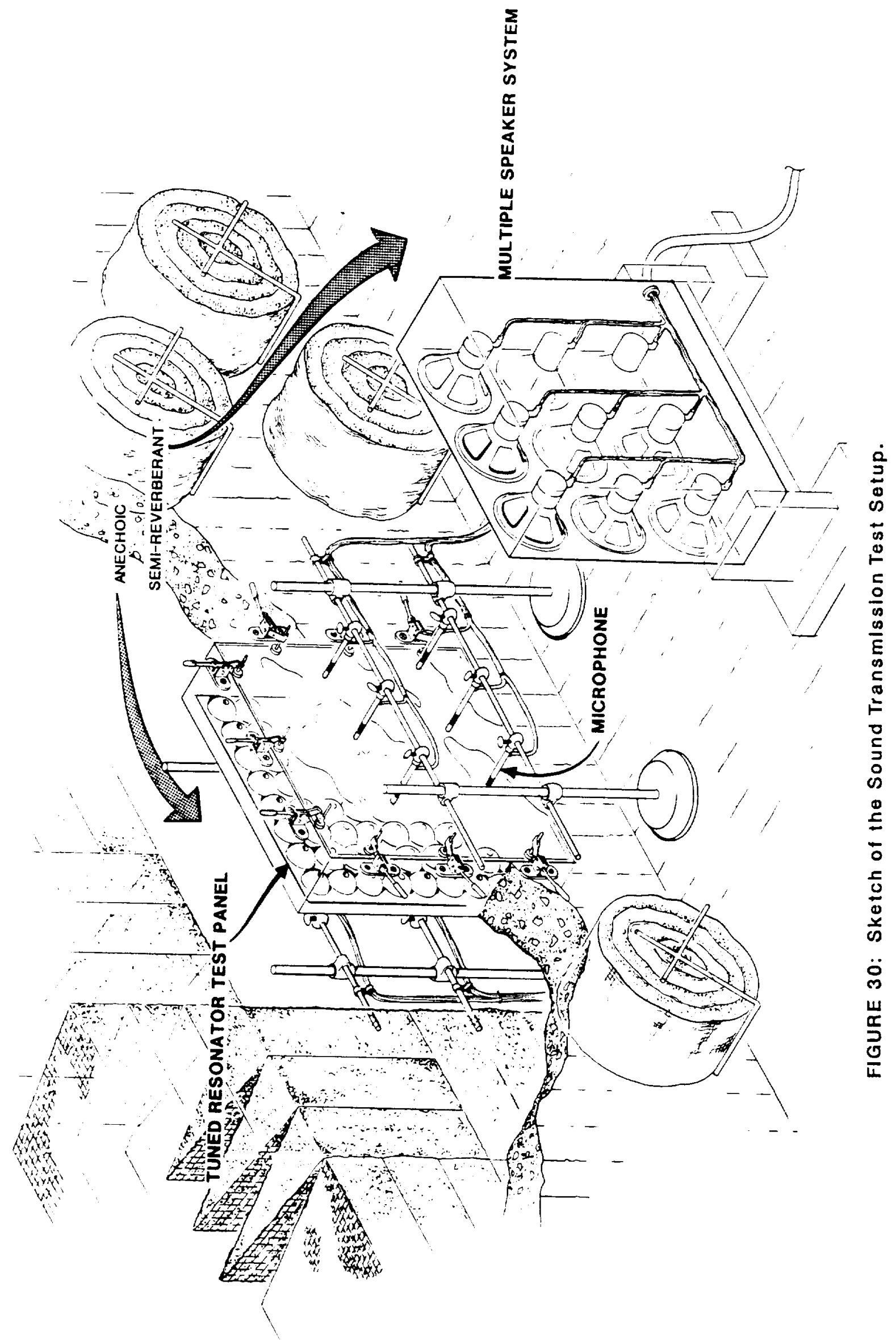




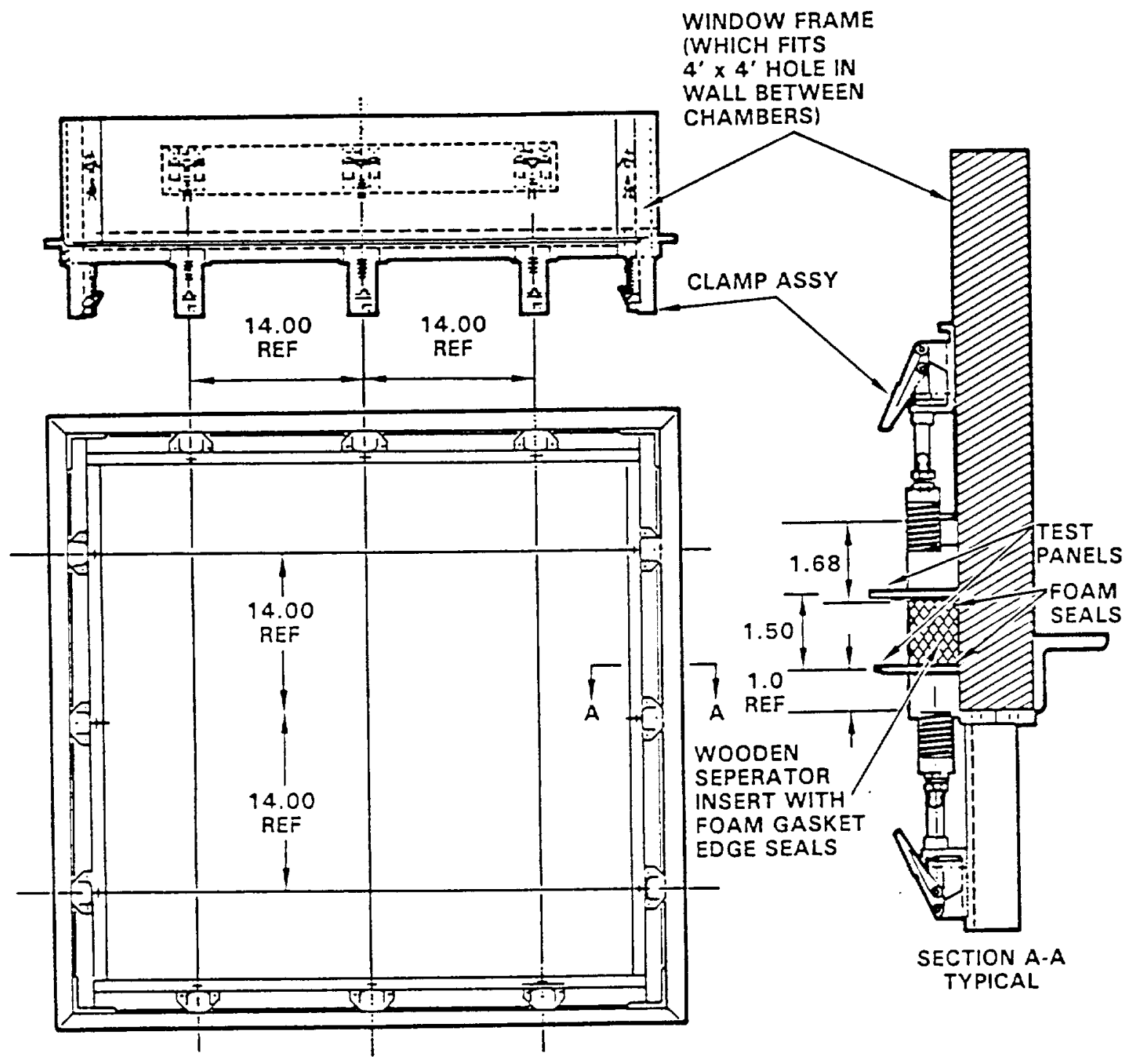

FIGURE 31: Transmission Loss Test Fixture (1.118 by $1.118 \mathrm{~m})$. 

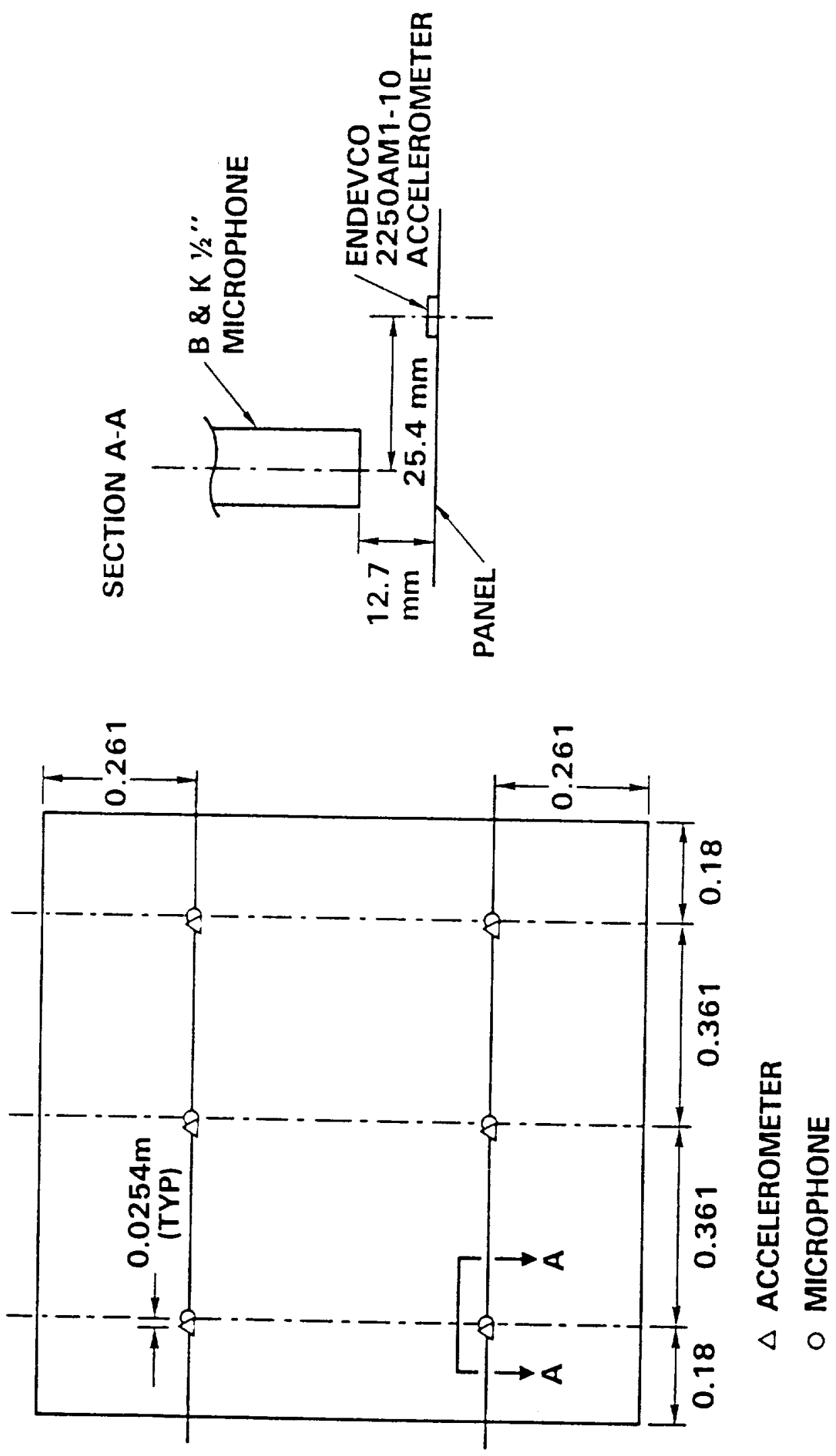

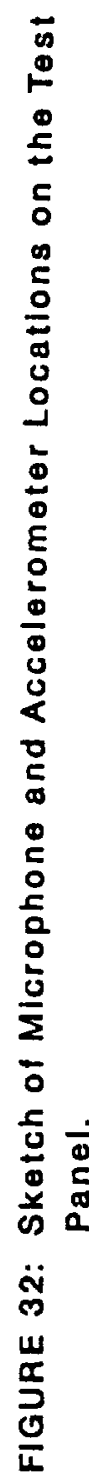




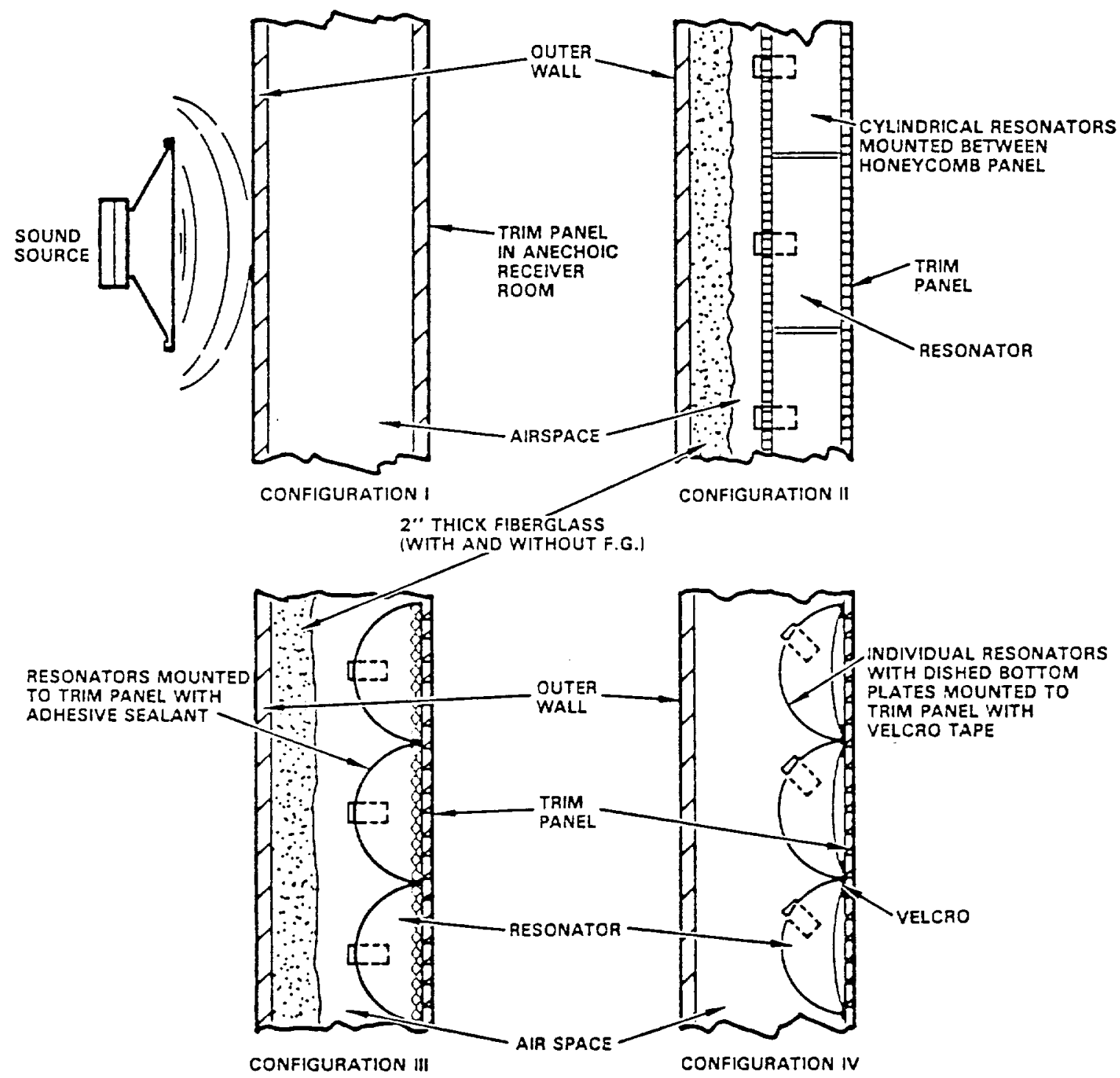

FIGURE 33: The Four Basic Double-Panel Wall Assembly Test Configurations. 


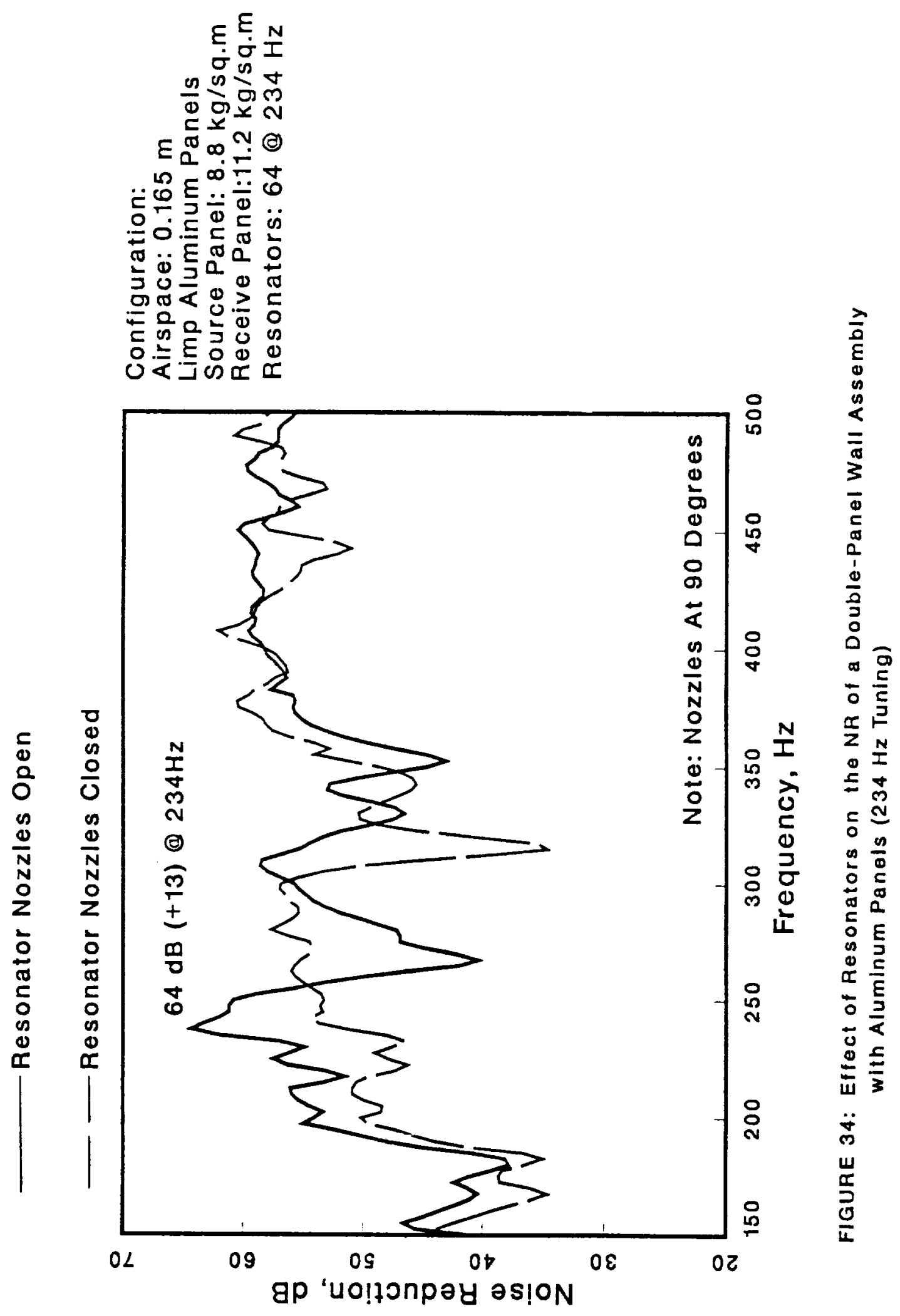




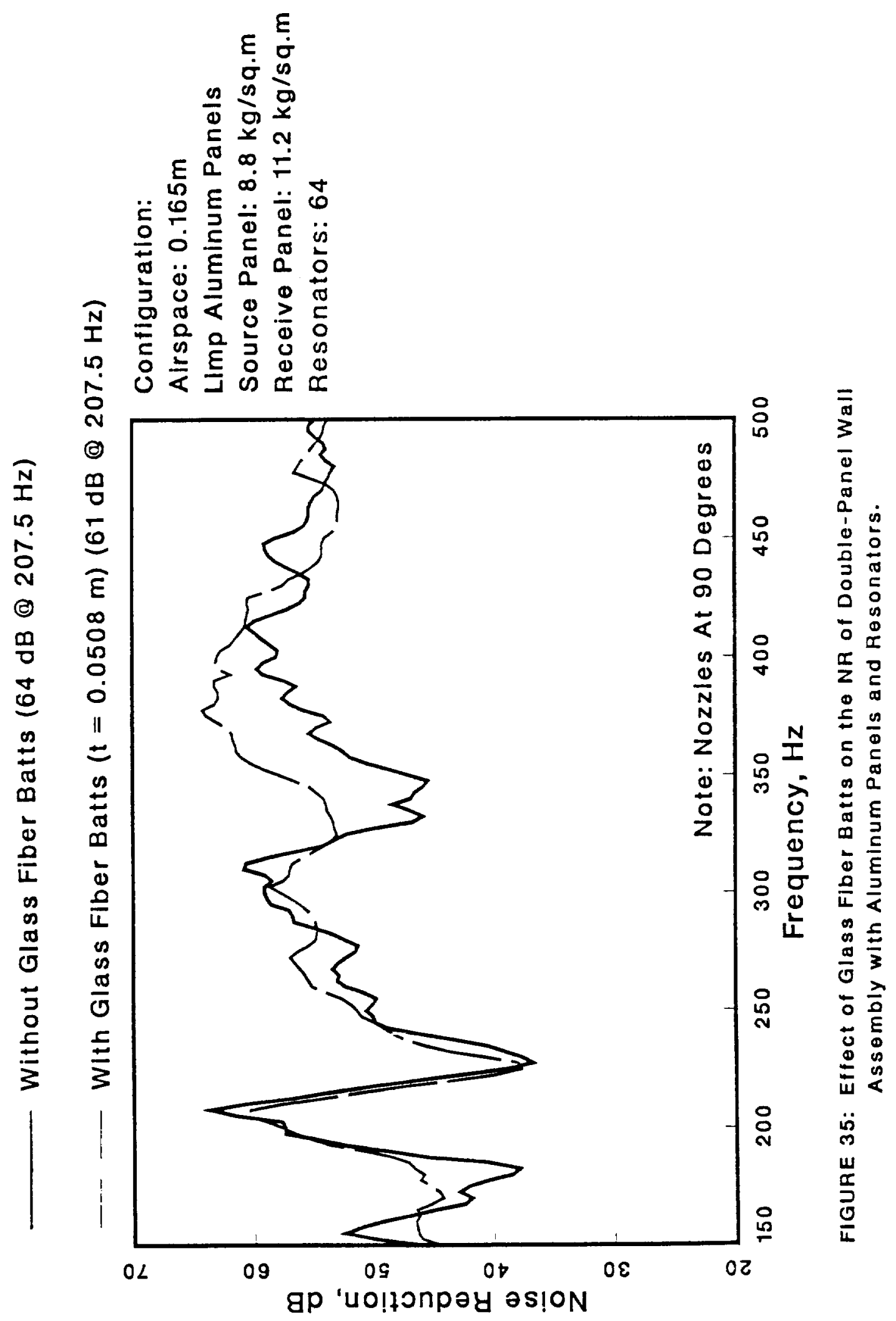




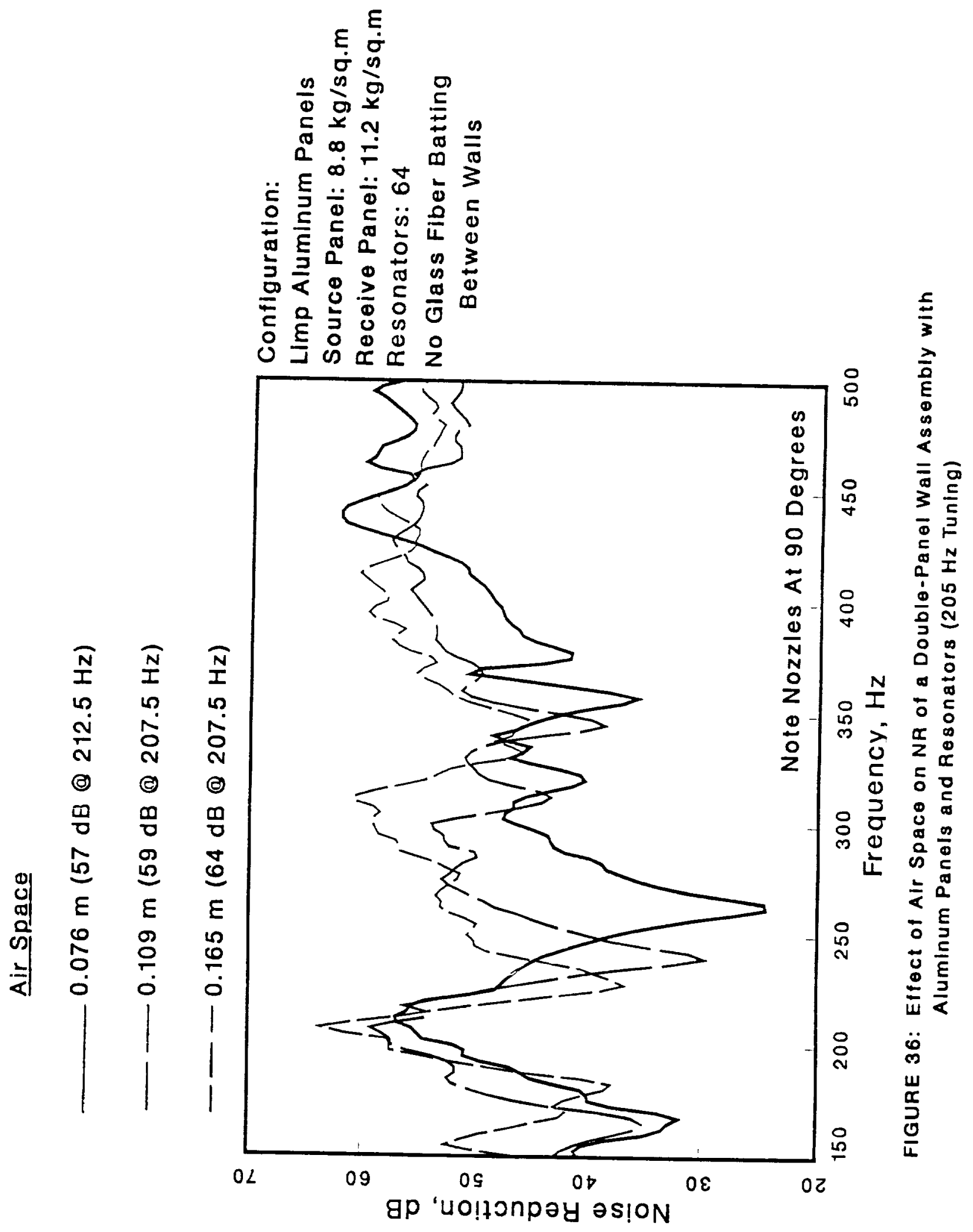




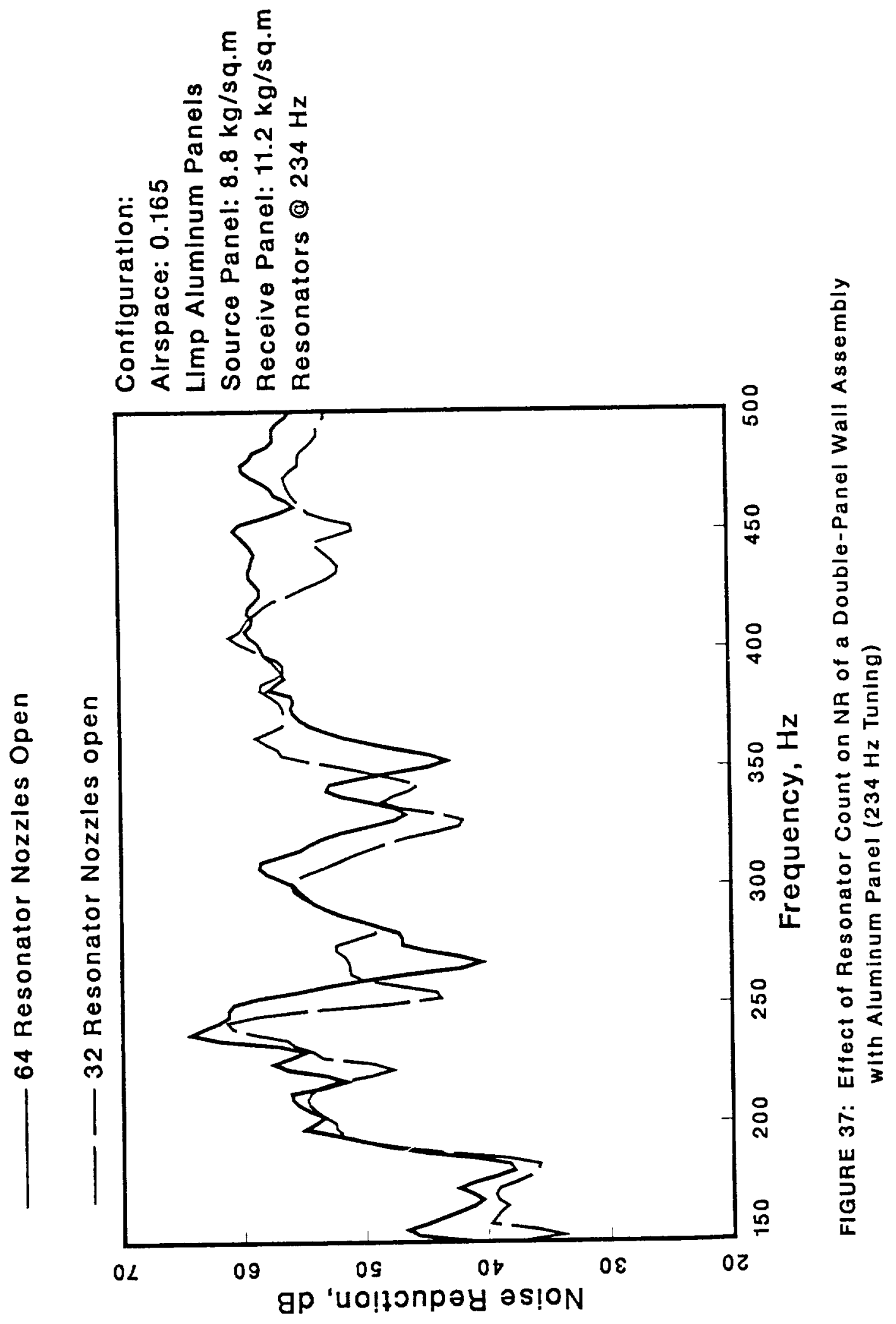




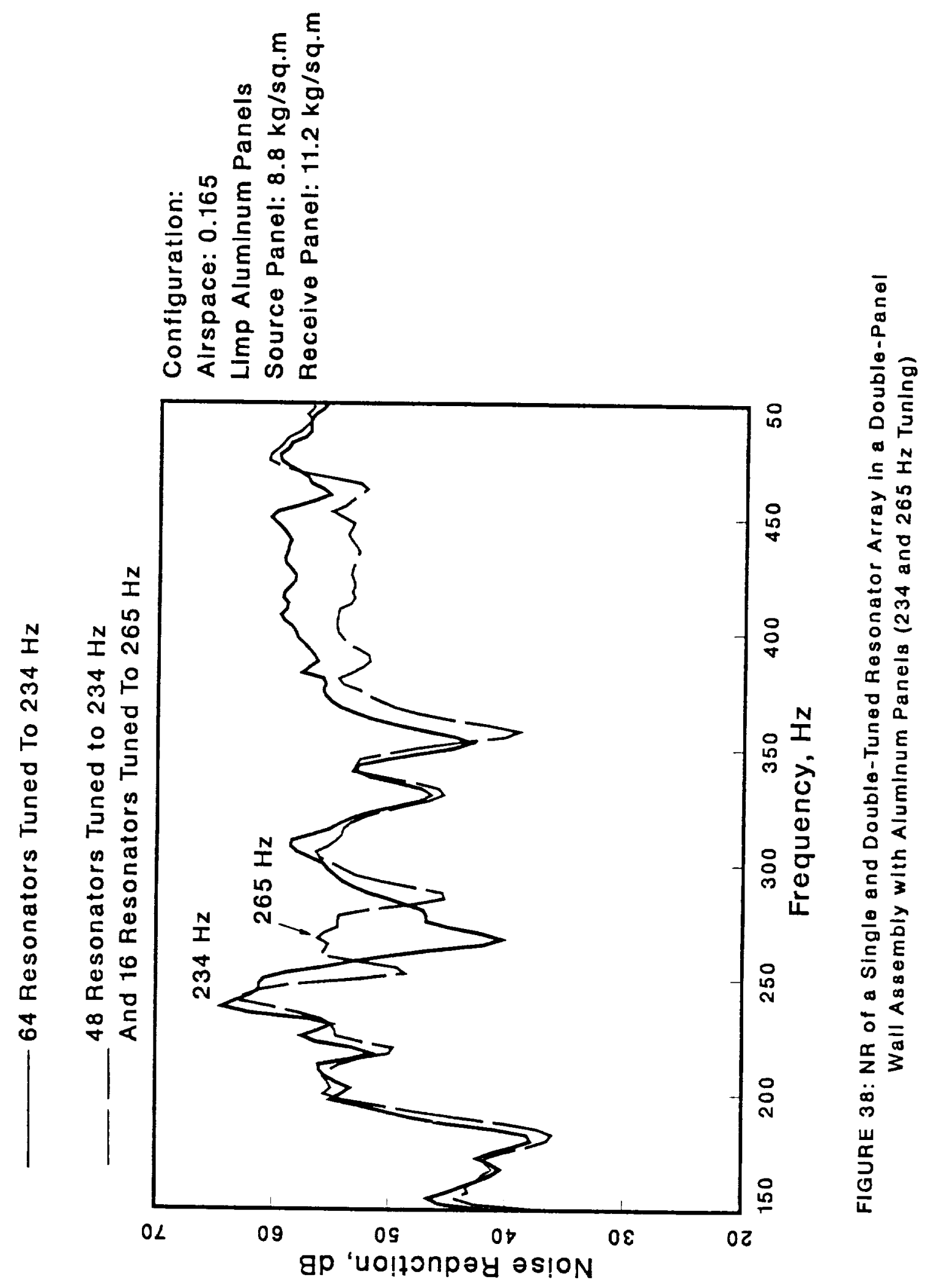




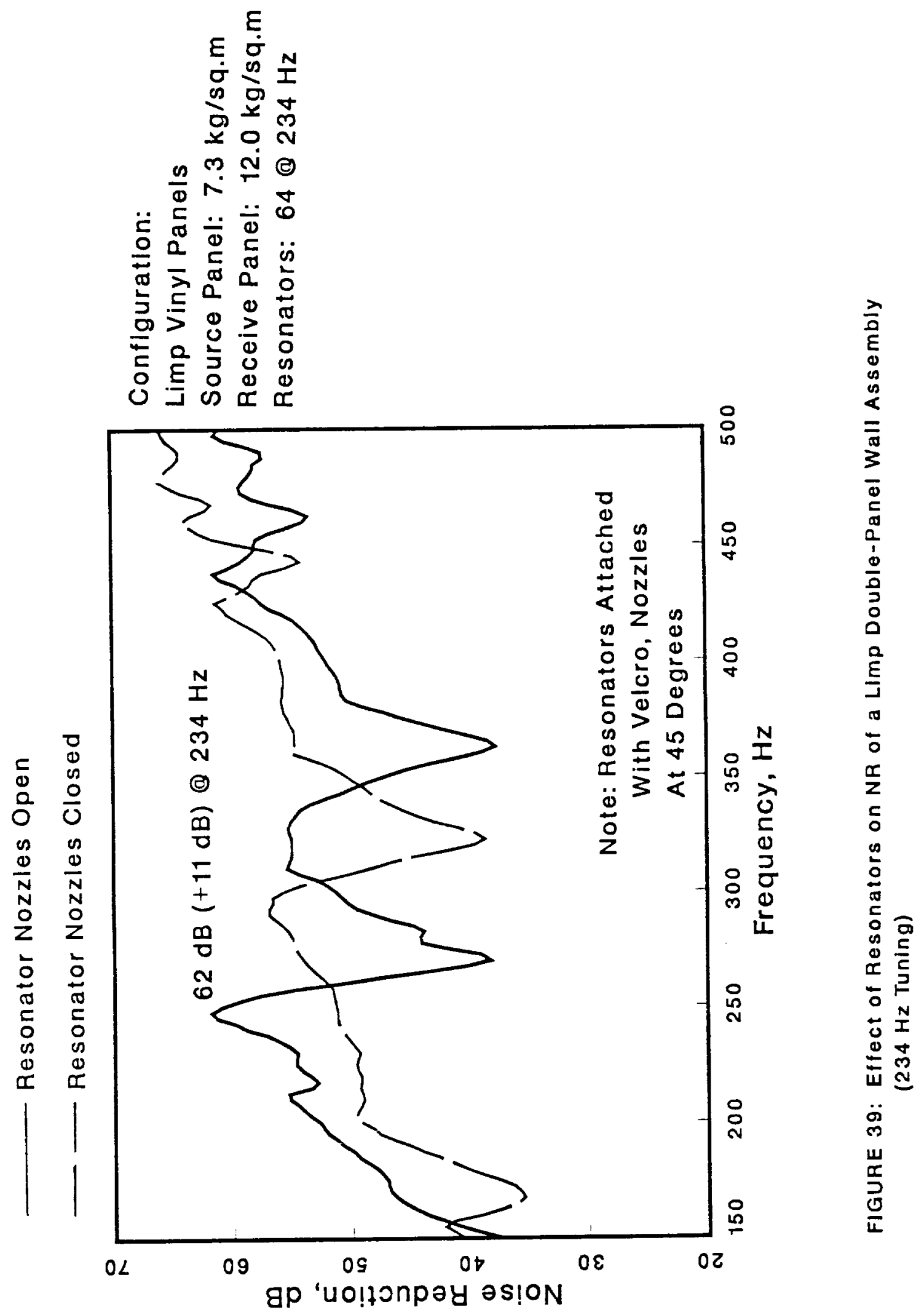




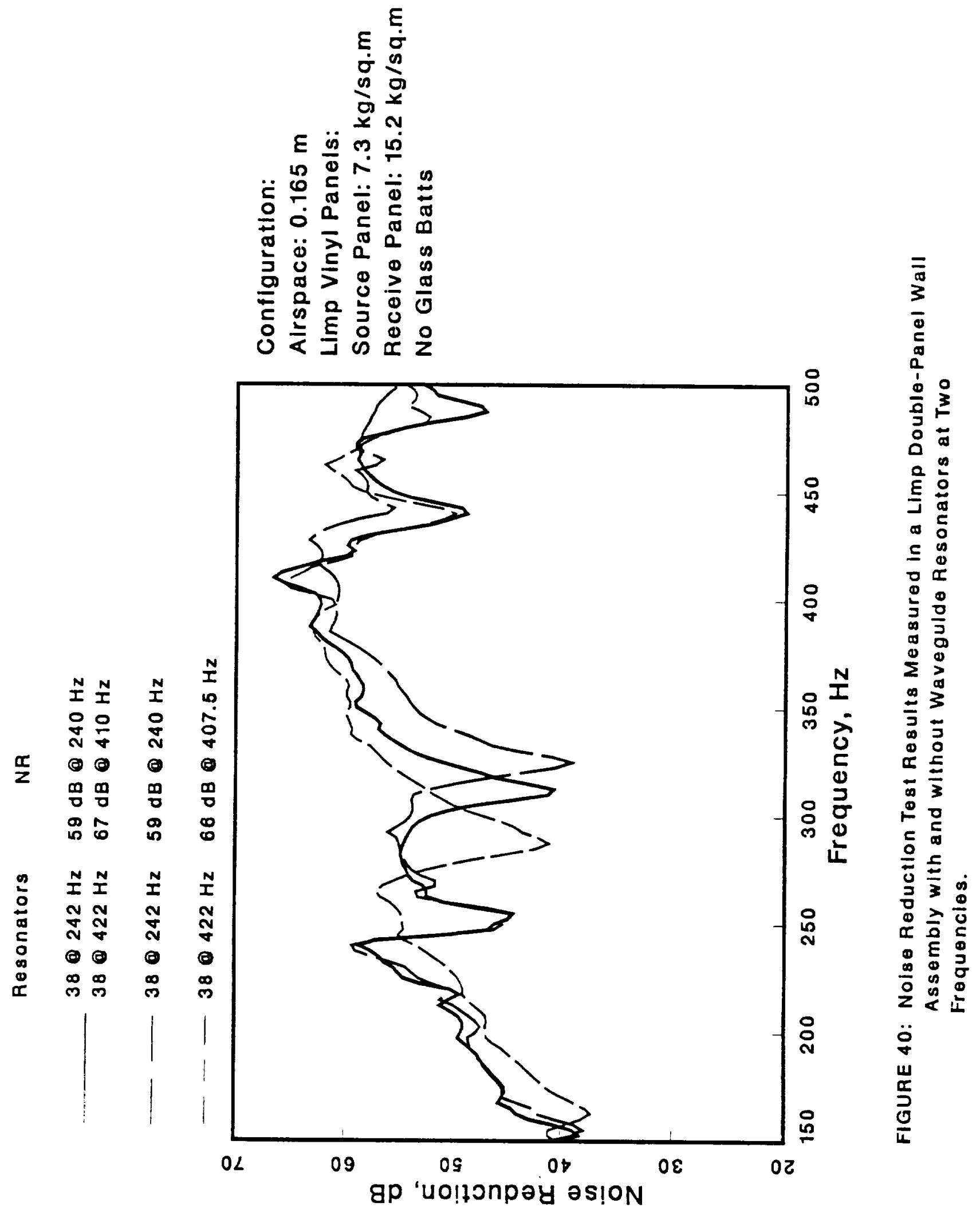




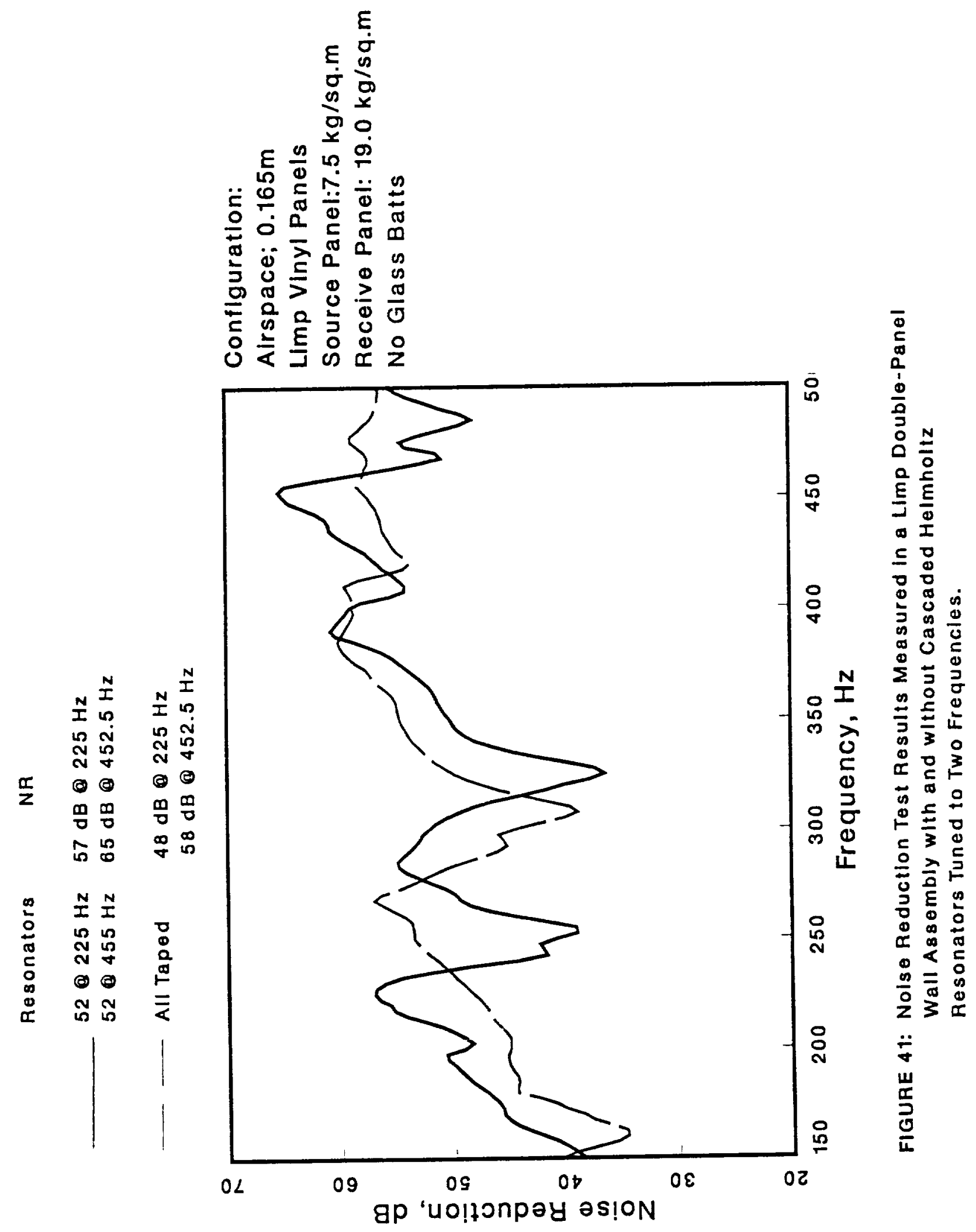




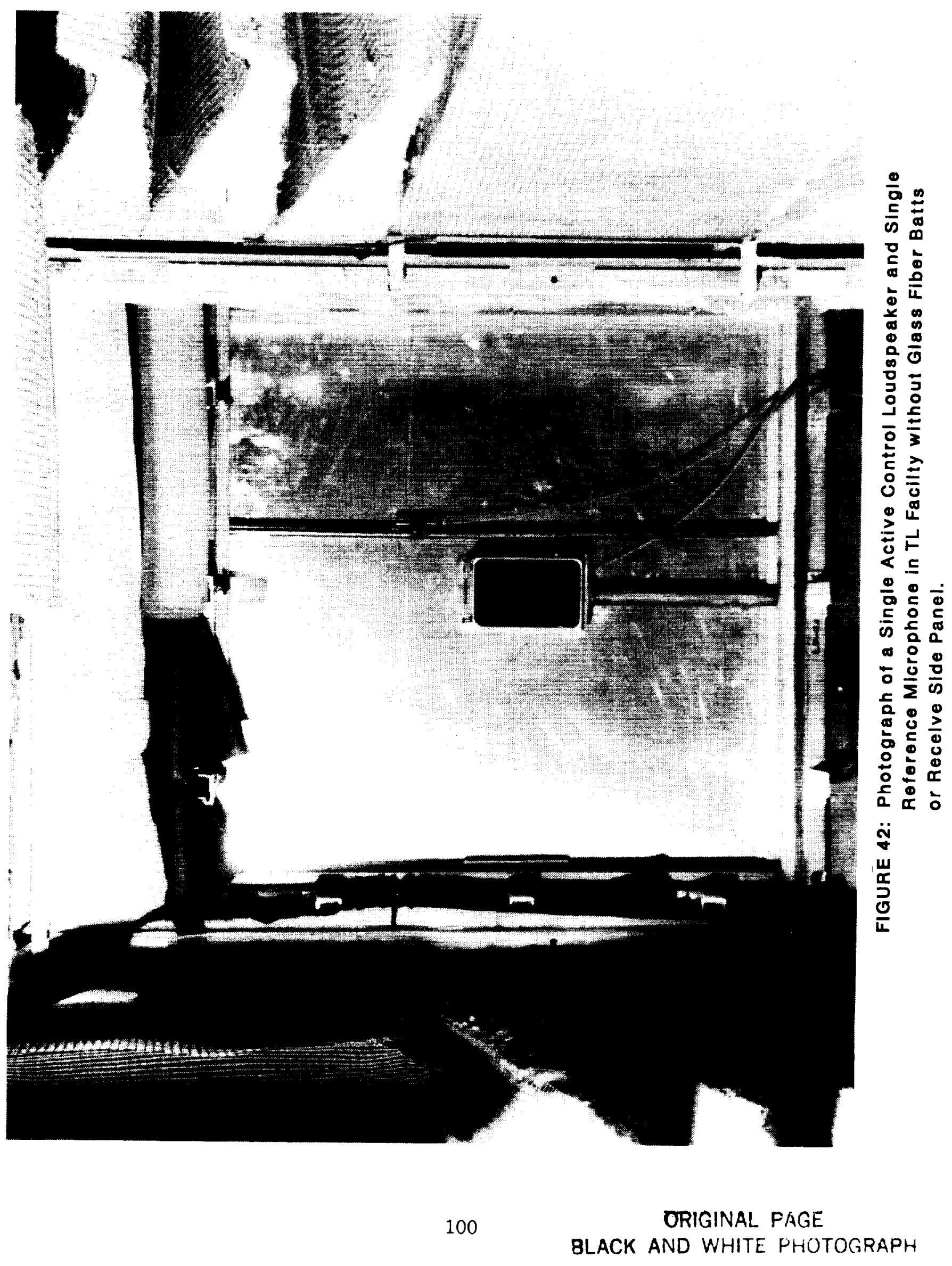




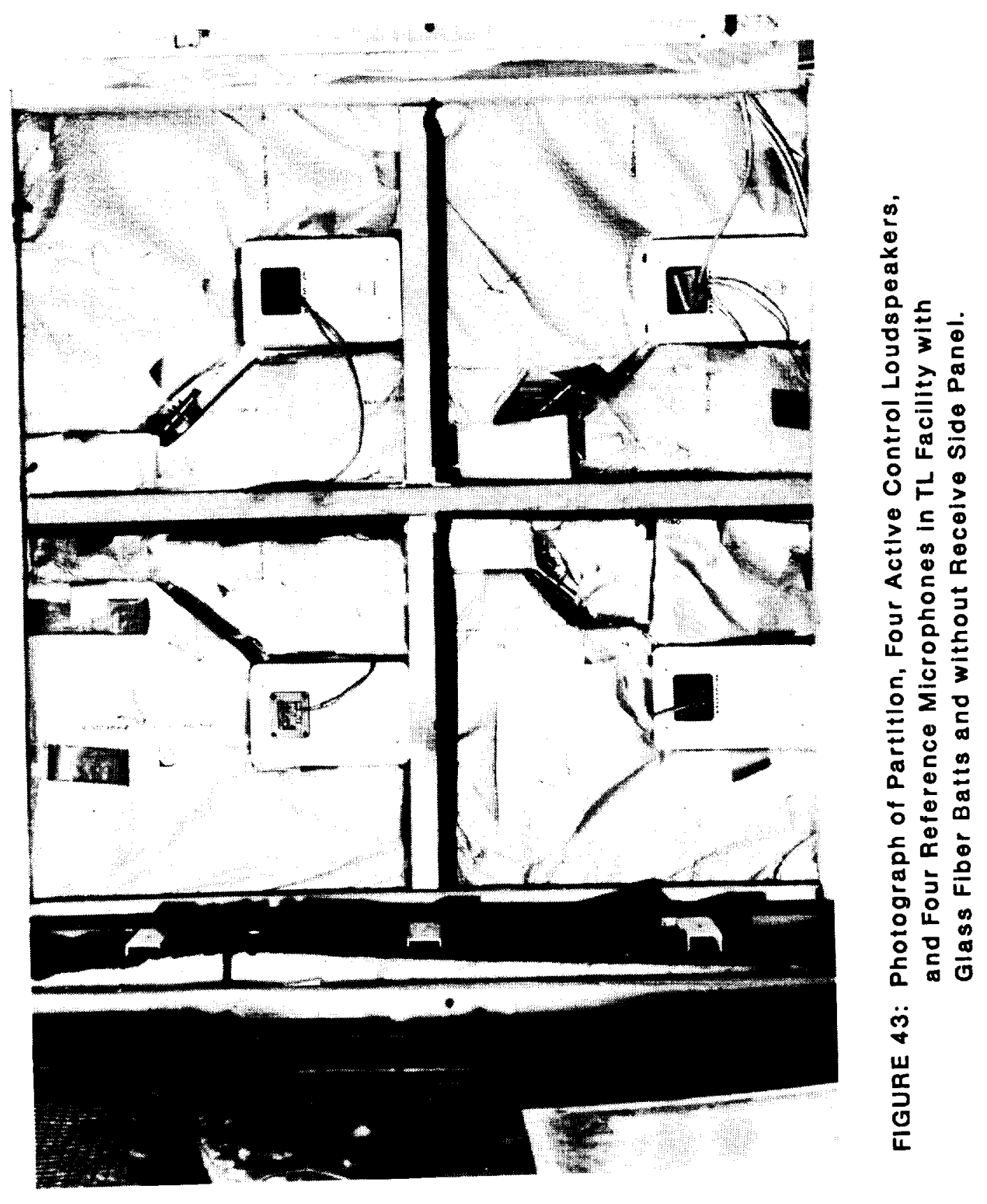

URMUAL Pa: 


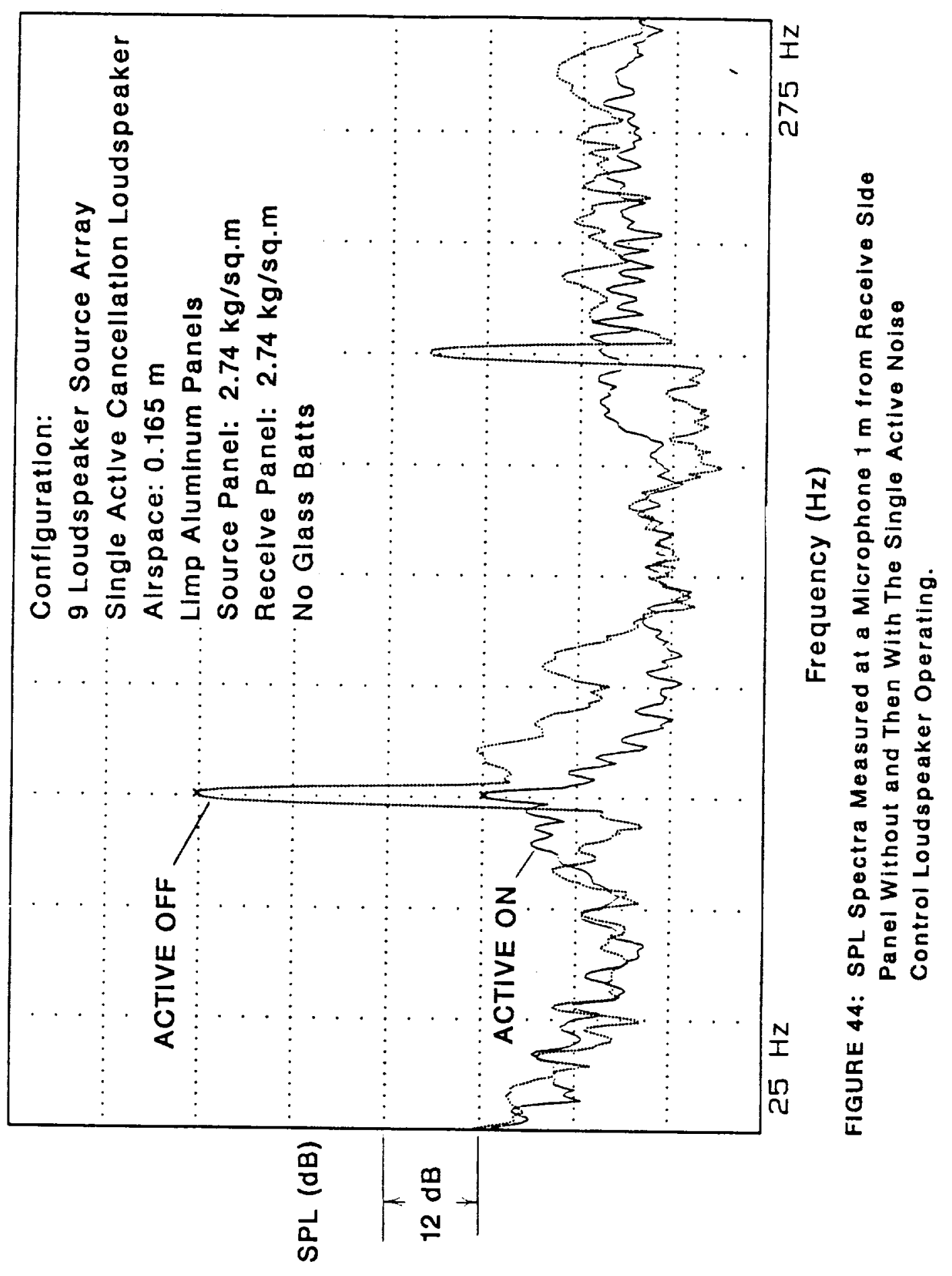




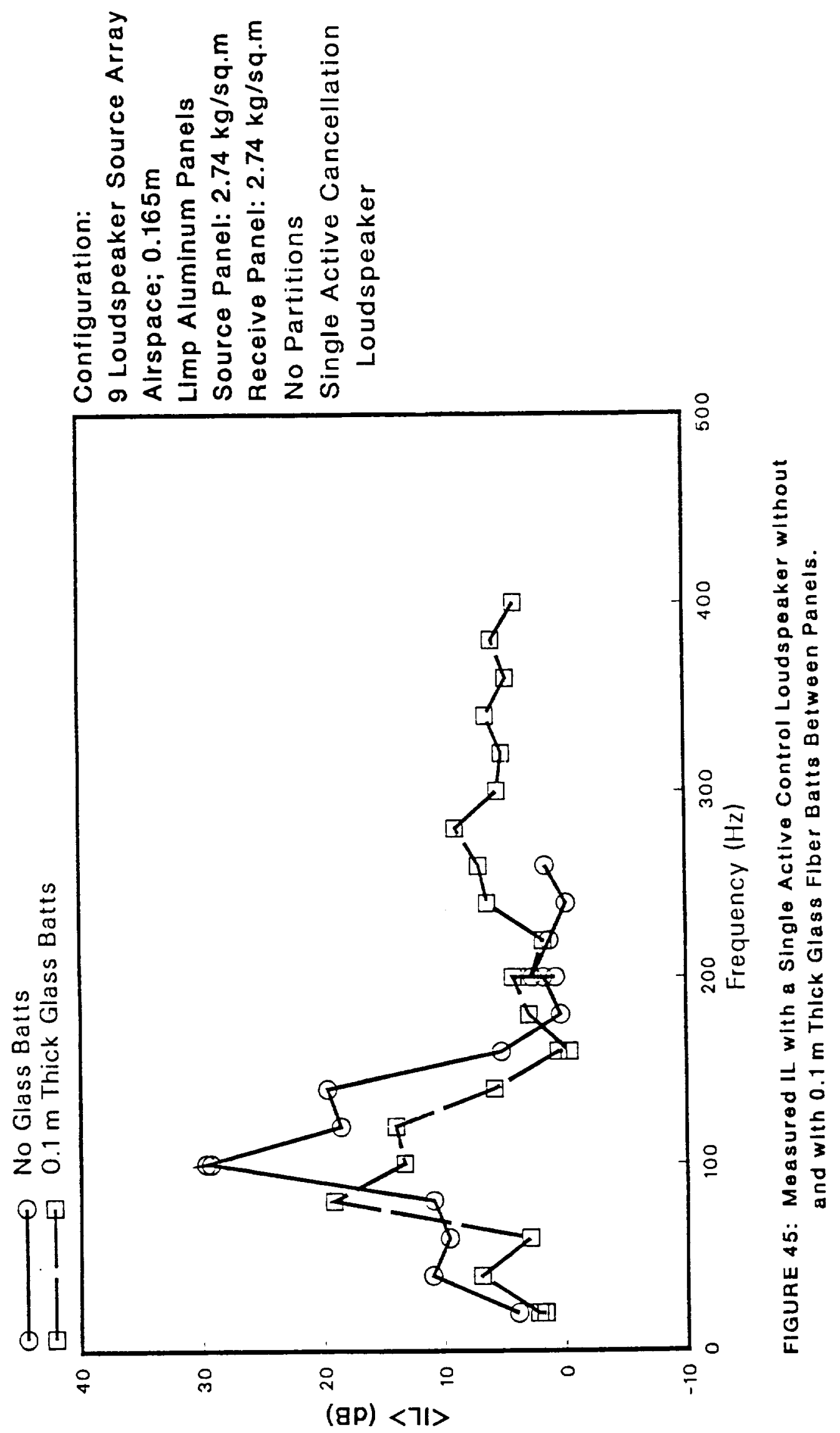




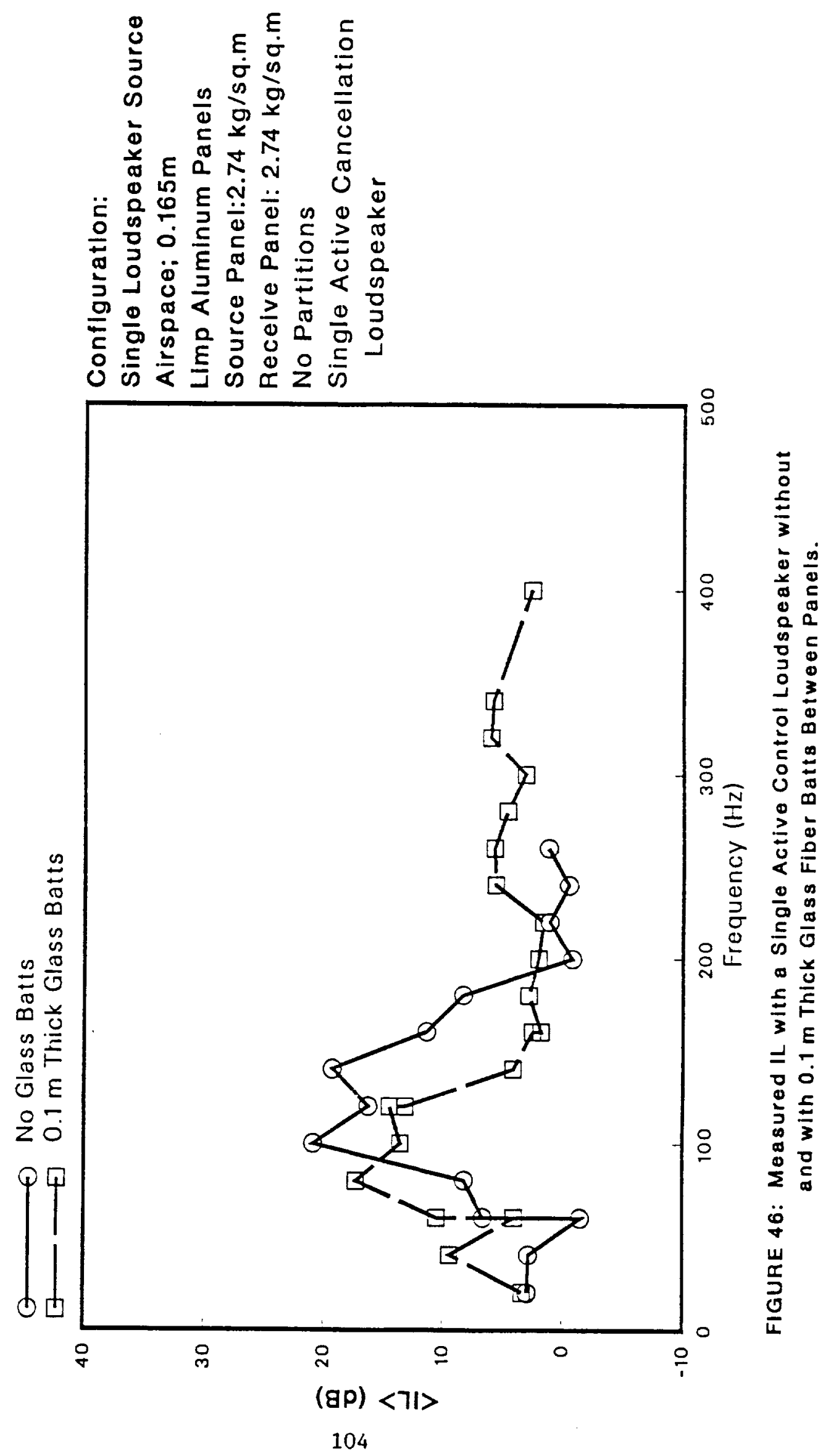




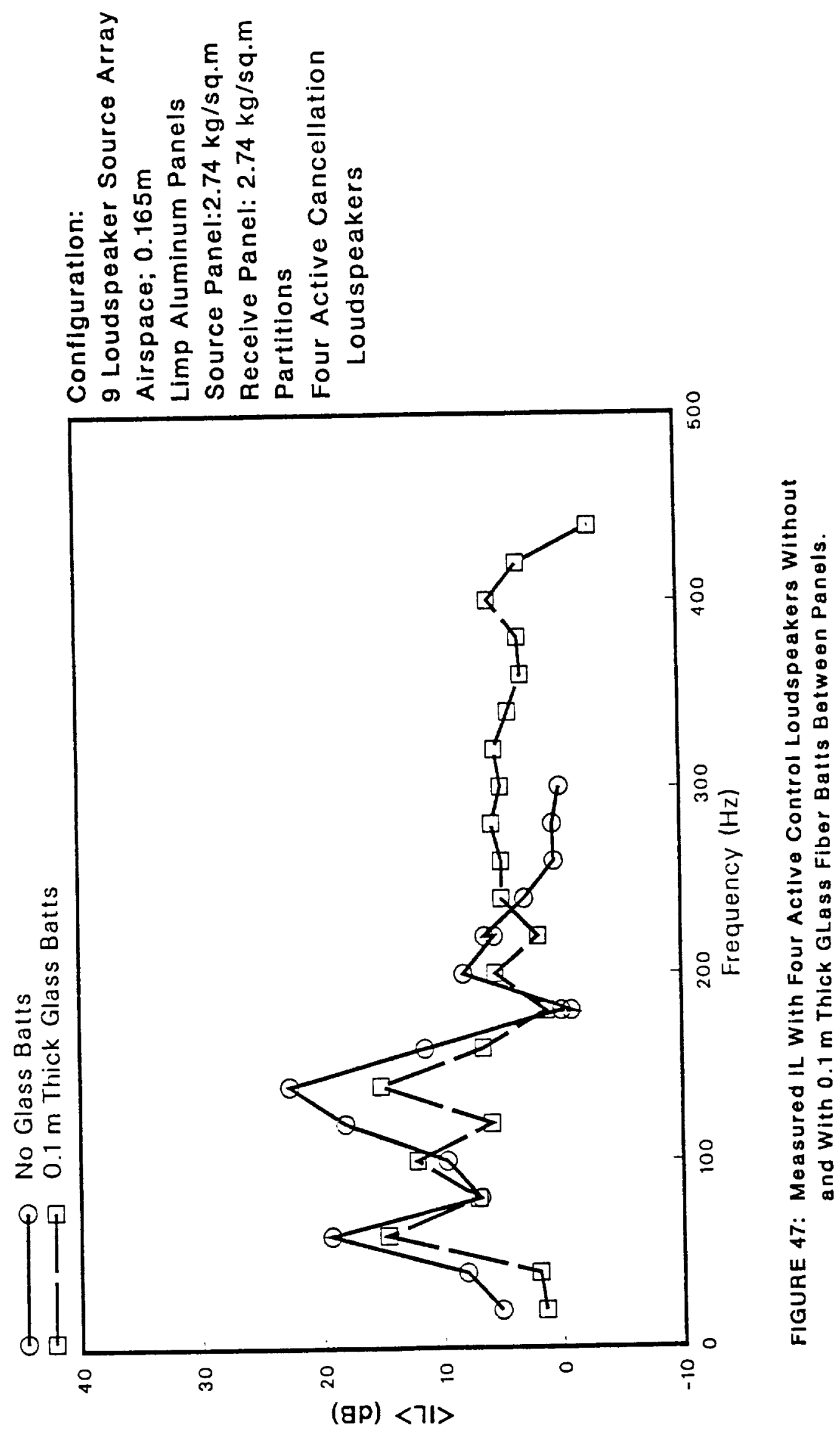




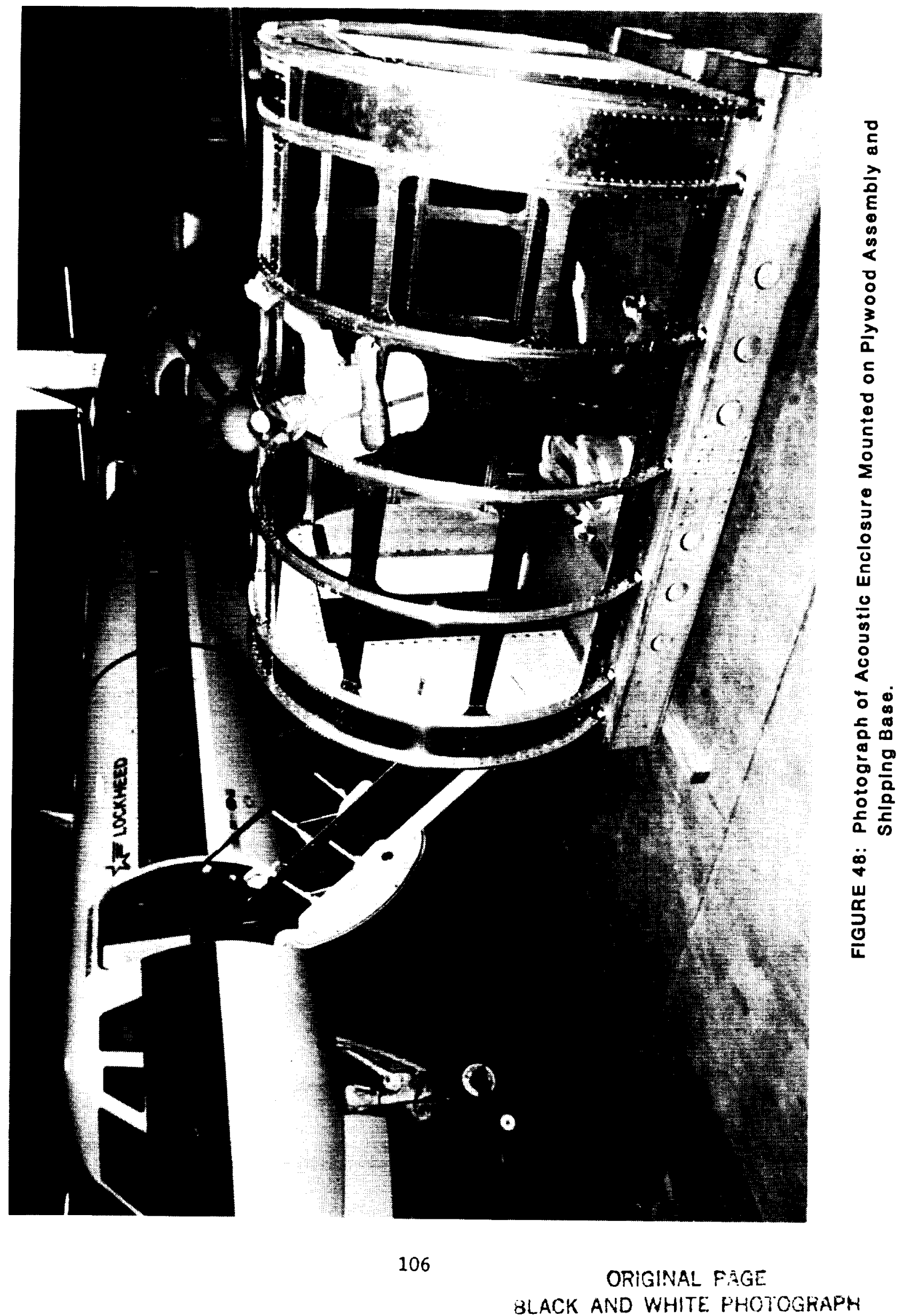




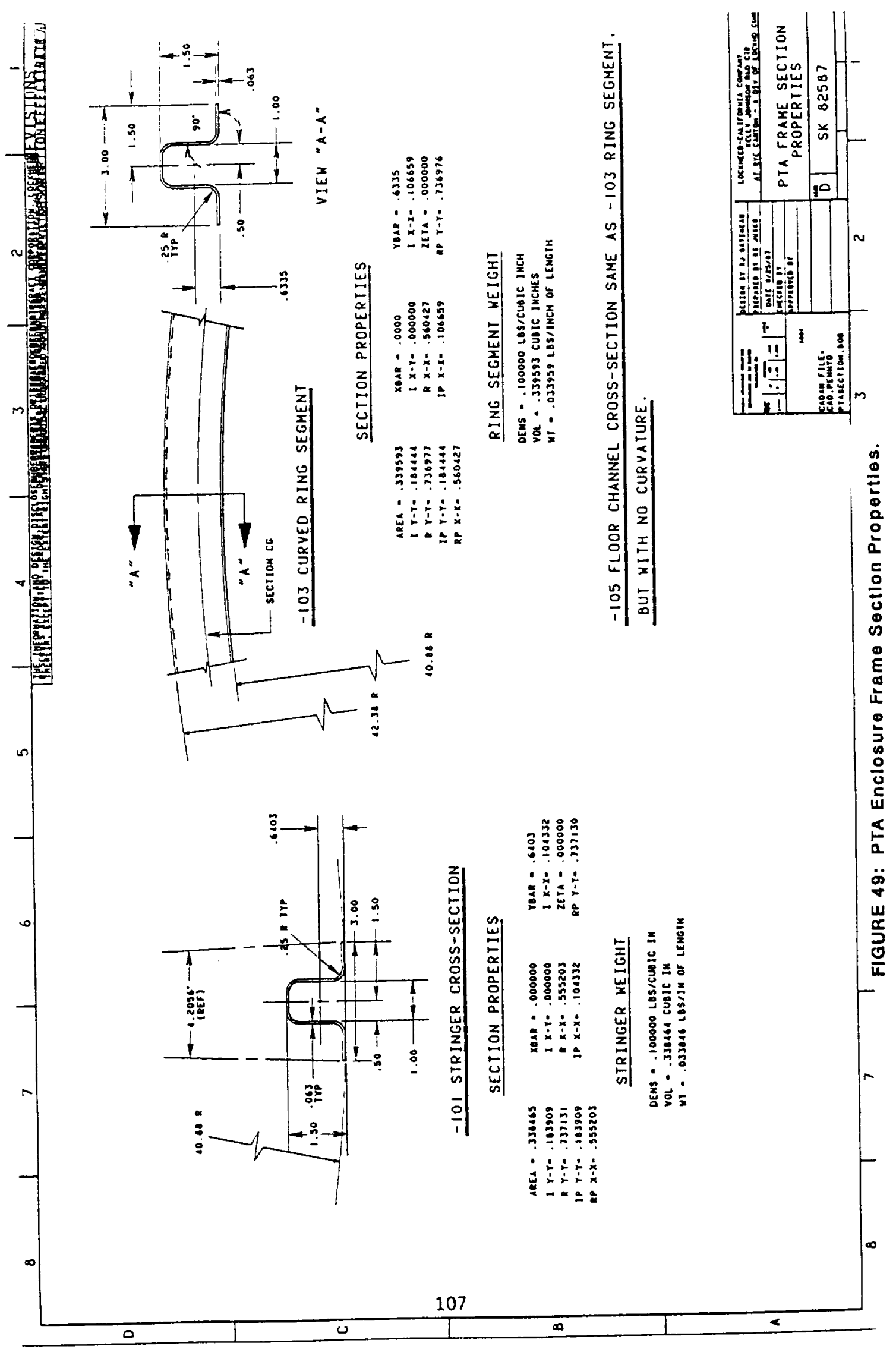




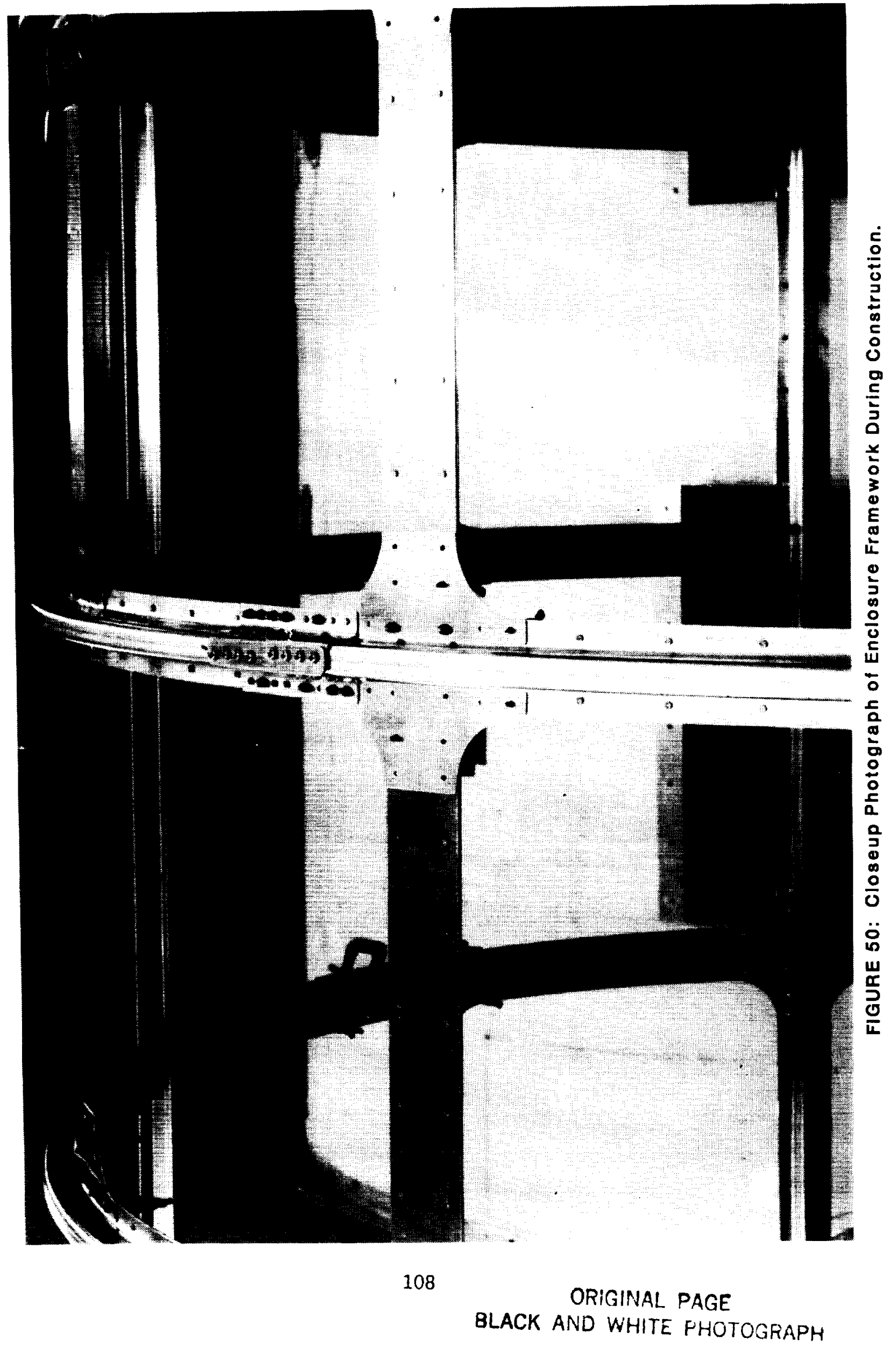




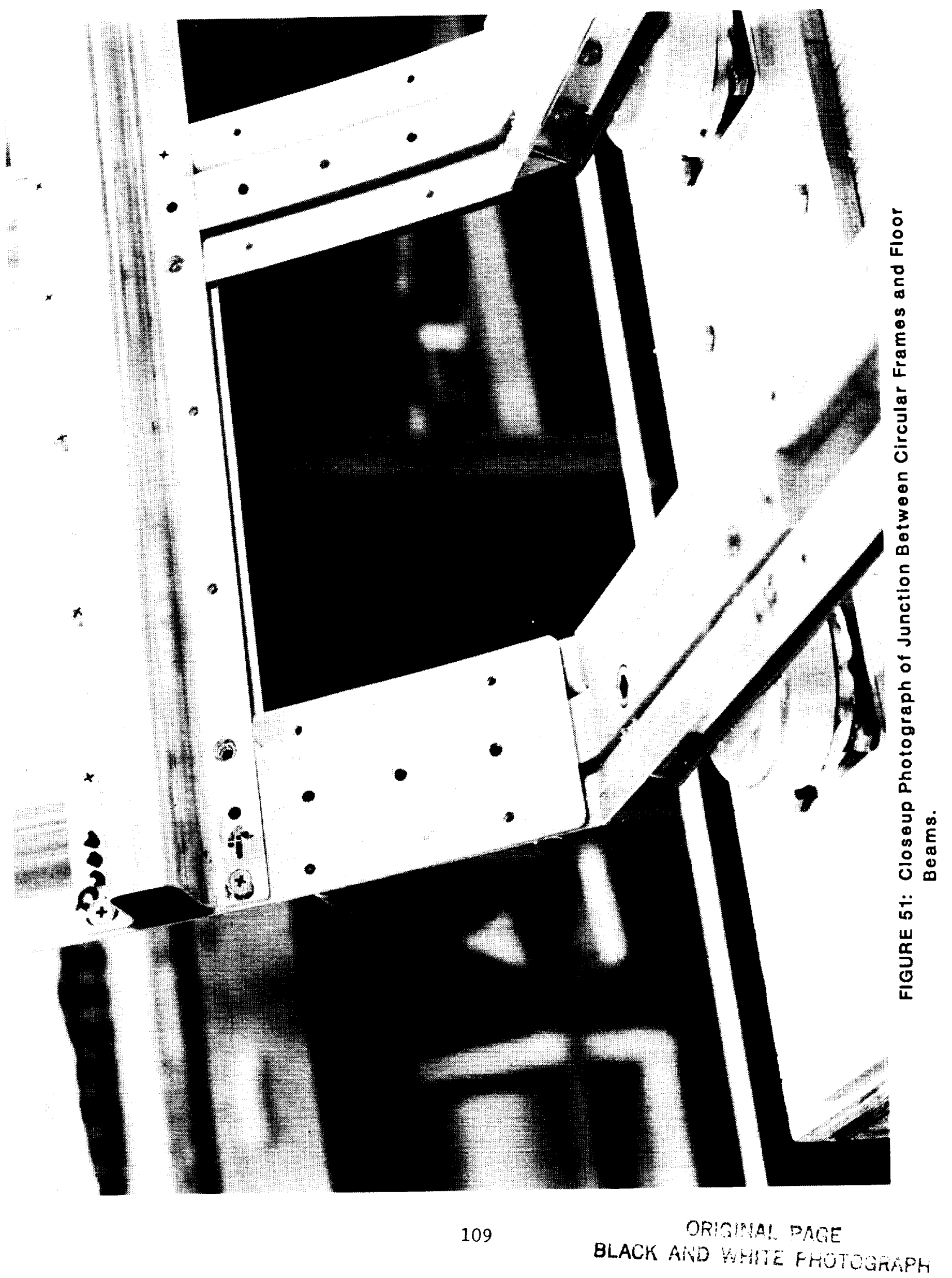




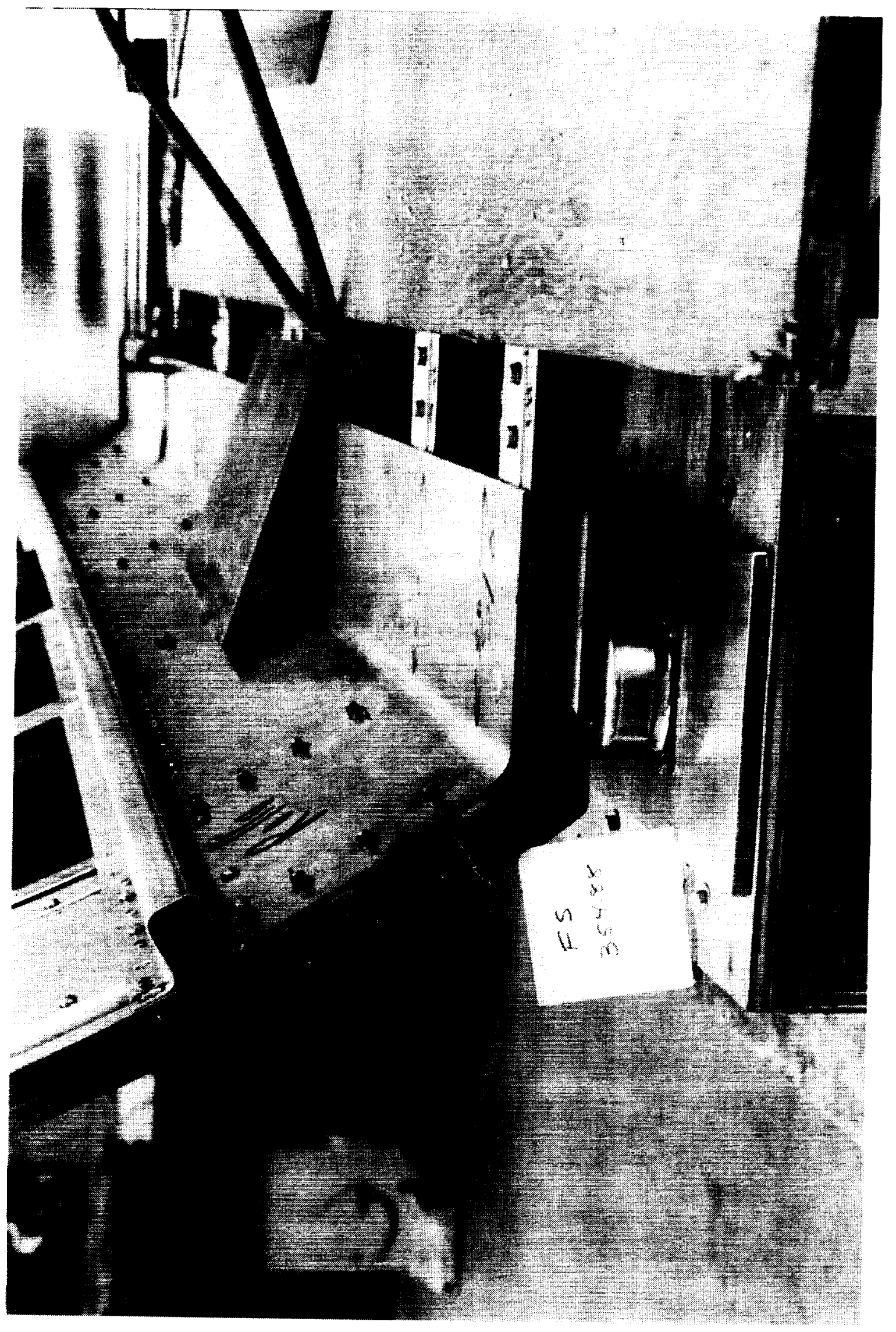

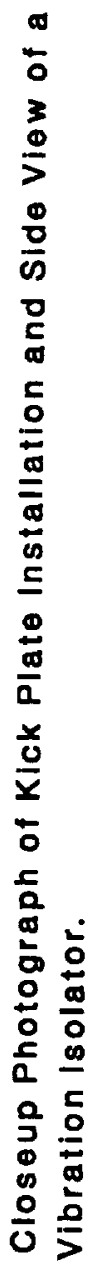

تัن

崩 

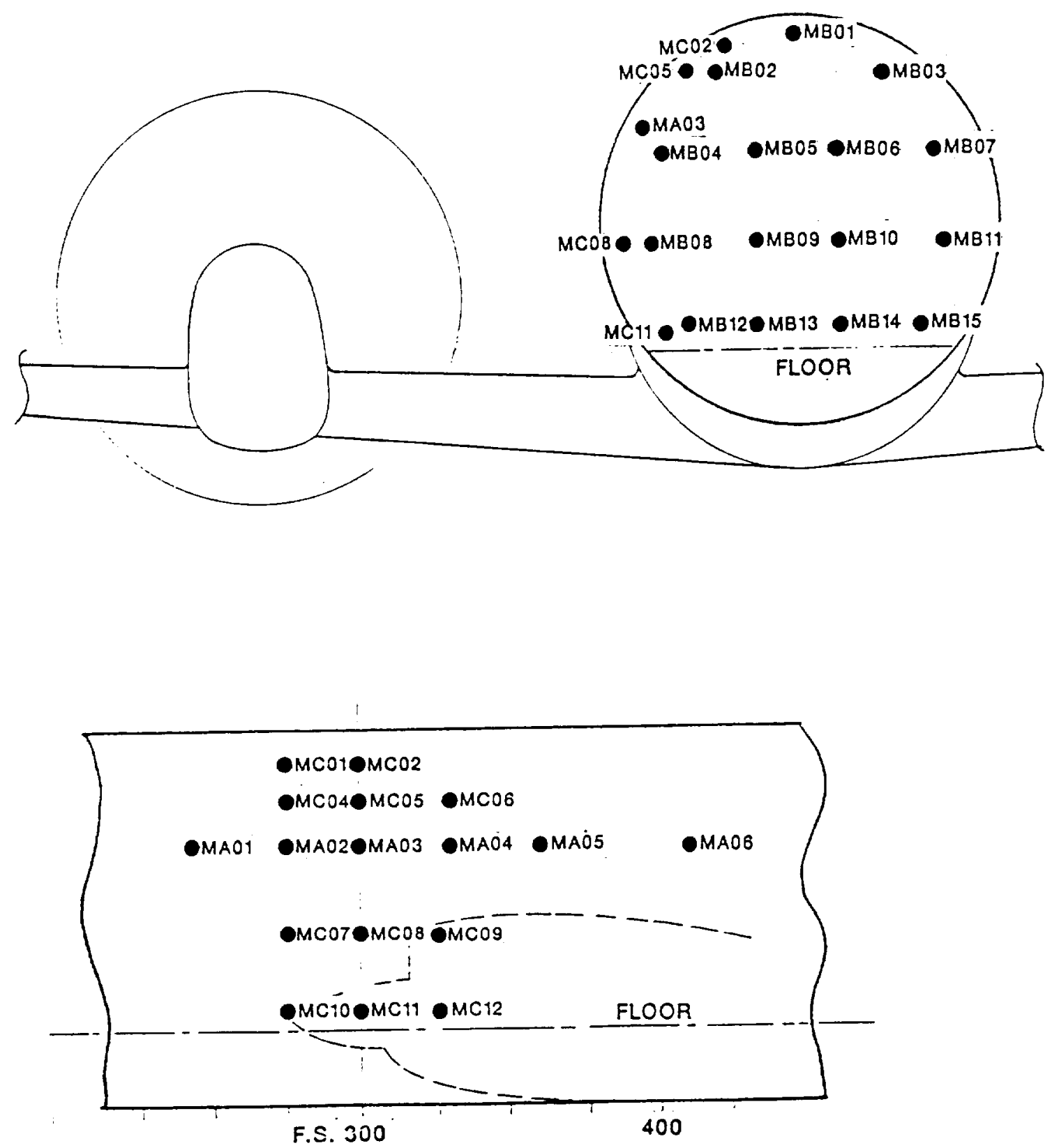

FIGURE 53: Cabin Microphone Designations and Locations Superimposed on Cabin Cross Sections. 

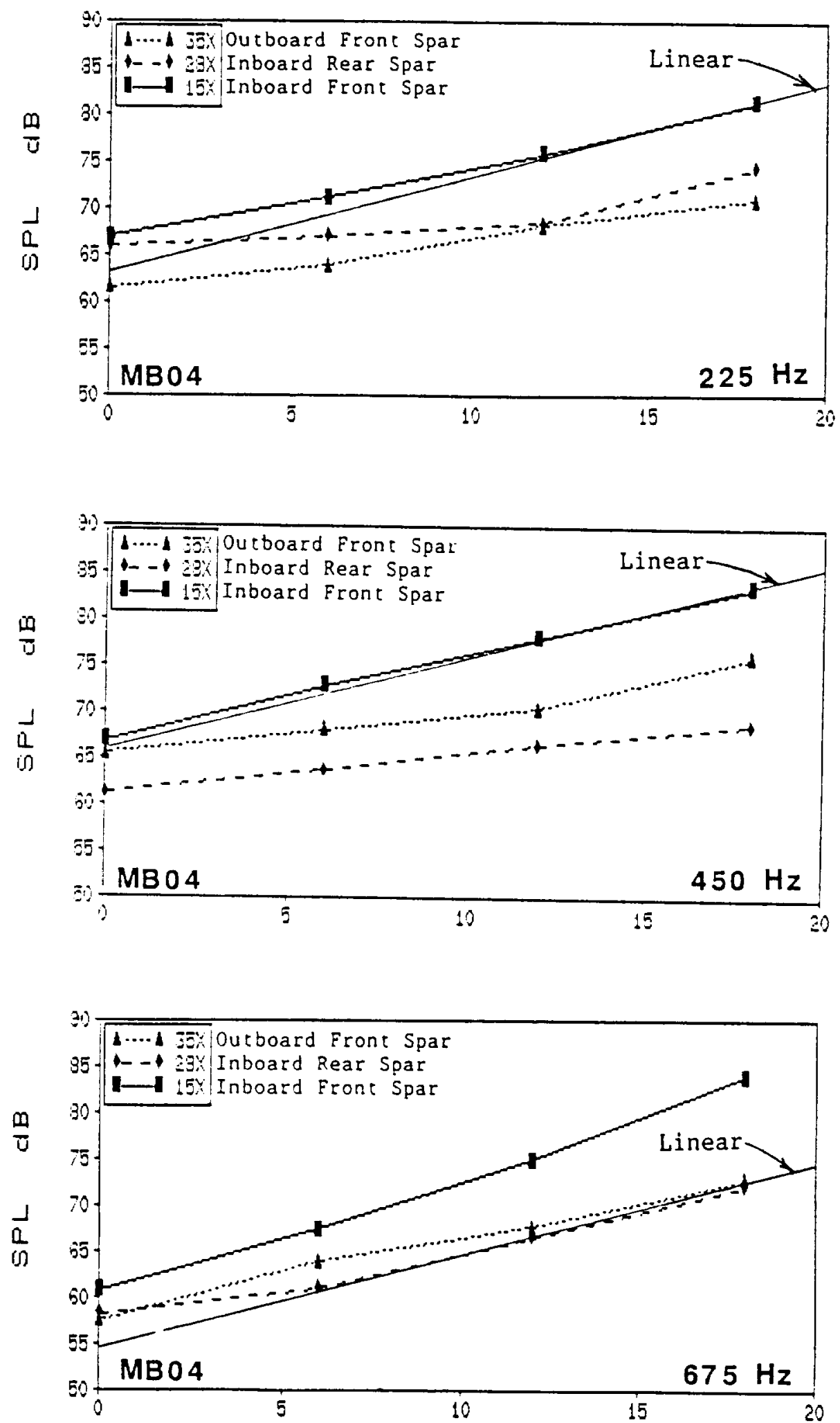

Relatlve Force Level, $d B$

FIGURE 54: Dependence of Tonal SPLs at Cabin Microphone in Prop Plane versus the Relative Input Force Level for the Three Force Locations in The Wing. 

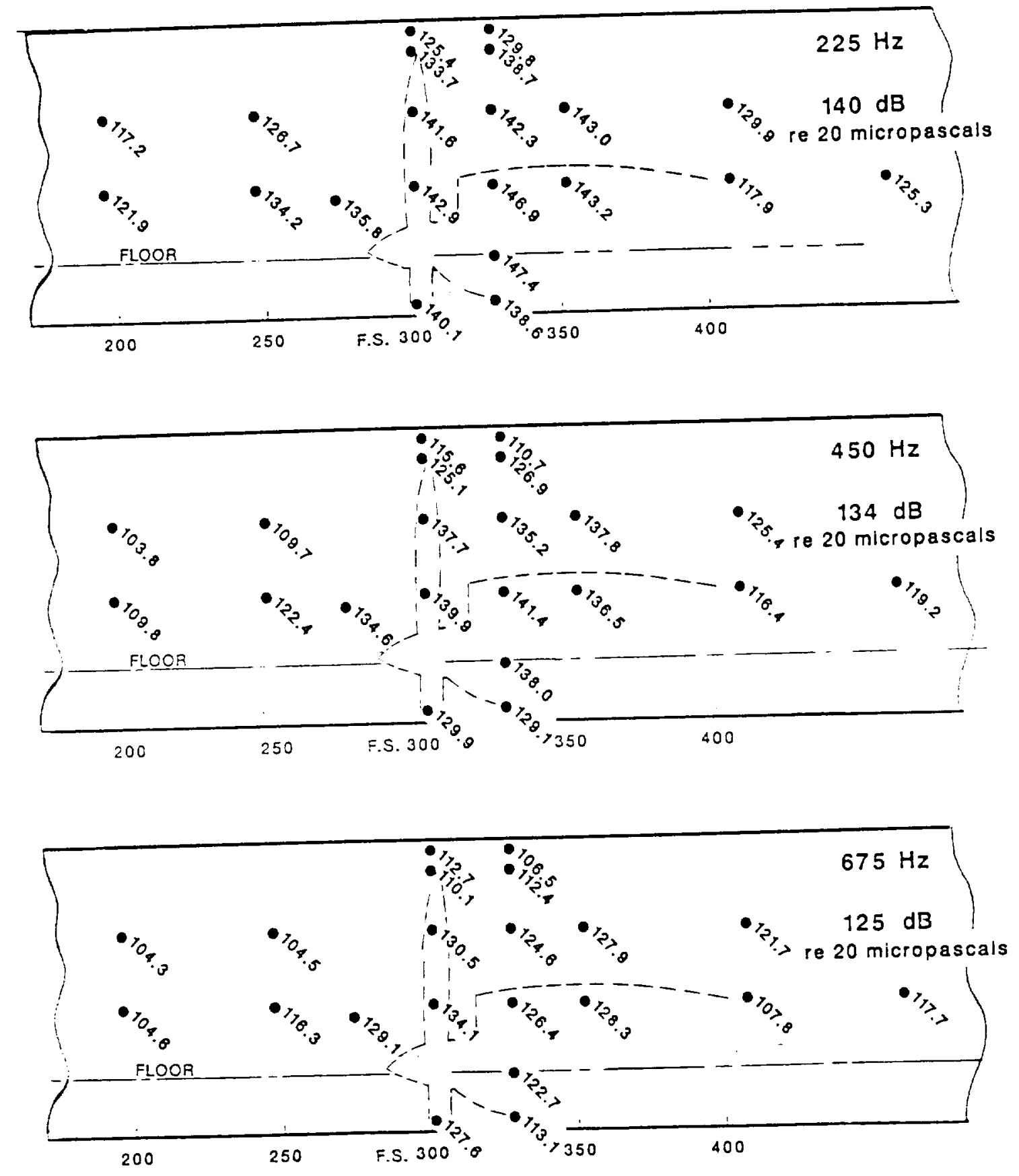

FIGURE 55: Fuselage SPLs Measured at the First Three Harmonics and at an Altltude of $10,700 \mathrm{~m}$ and Speed of $0.8 \mathrm{M}$. 


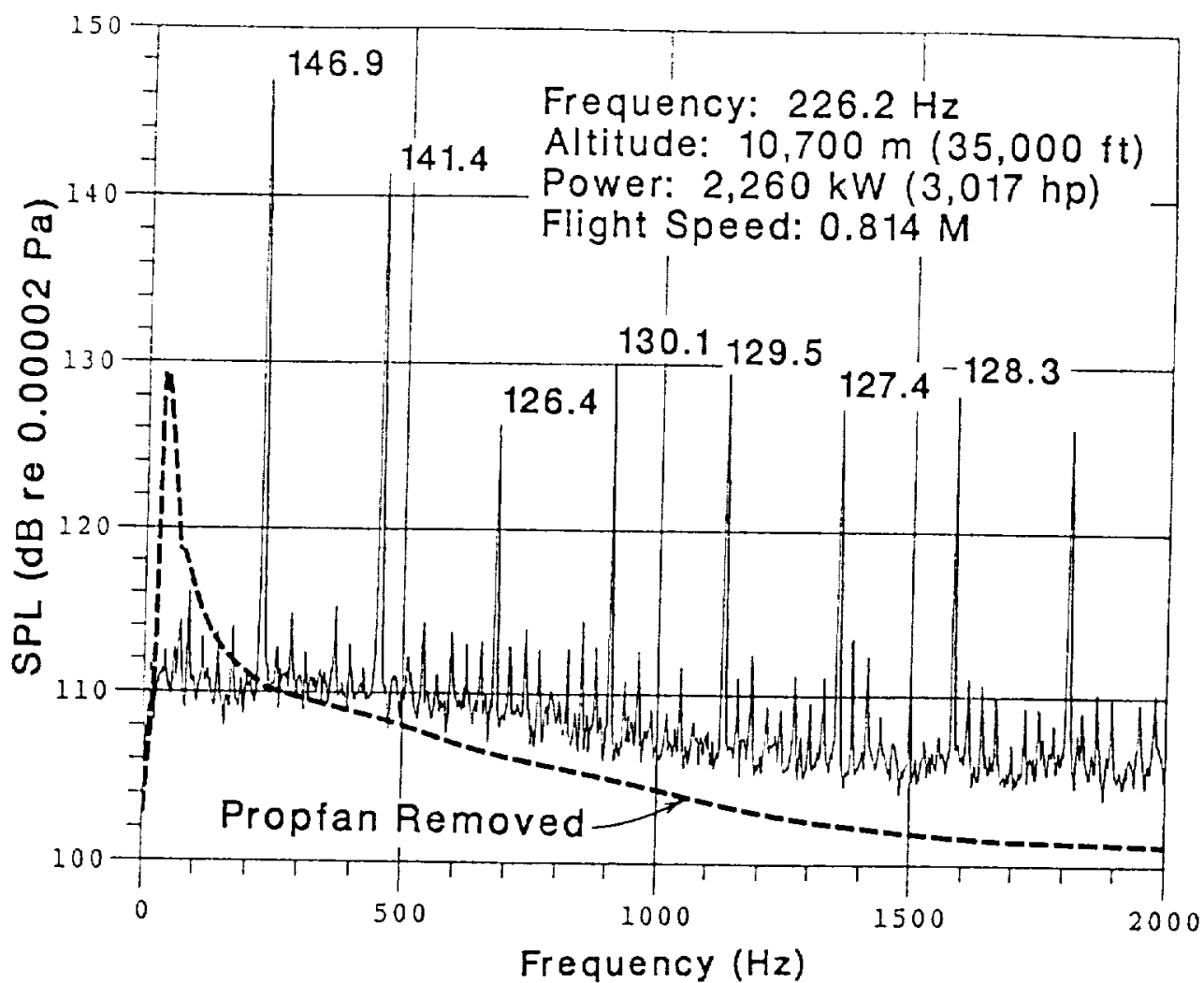

FIGURE 56: Fuselage SPL Spectra Measured $63.5 \mathrm{~cm}$ Behind the Prop Plane With and Without the Proptan at an Altitude of $10,700 \mathrm{~m}$ and Speed $0.8 \mathrm{M}$.

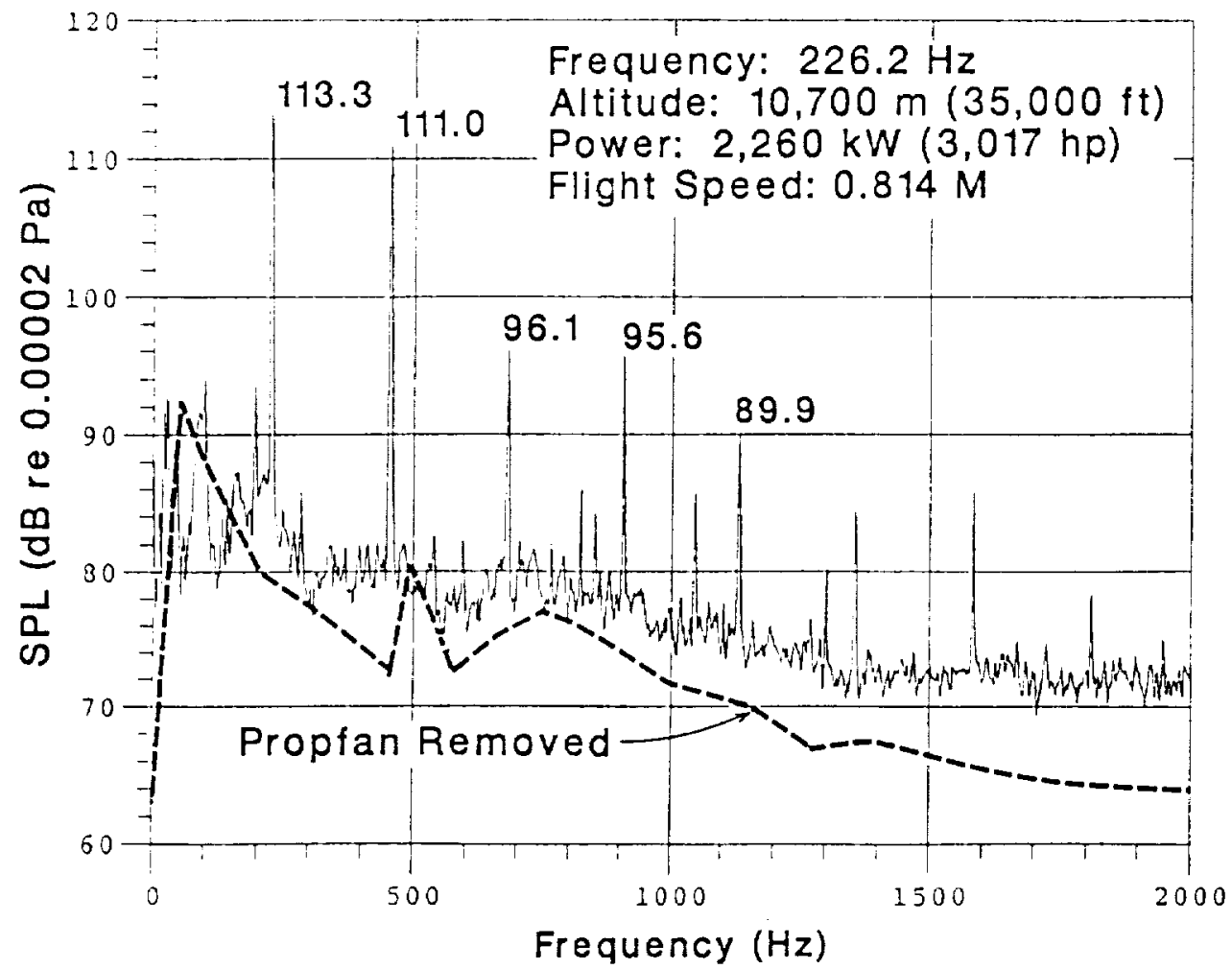

FIGURE 57: Cabin SPL Spectra Measured In the Prop Plane With and Without the Propfan at an Altltude of $10,700 \mathrm{~m}$ and Speed of $0.8 \mathrm{M}$. 

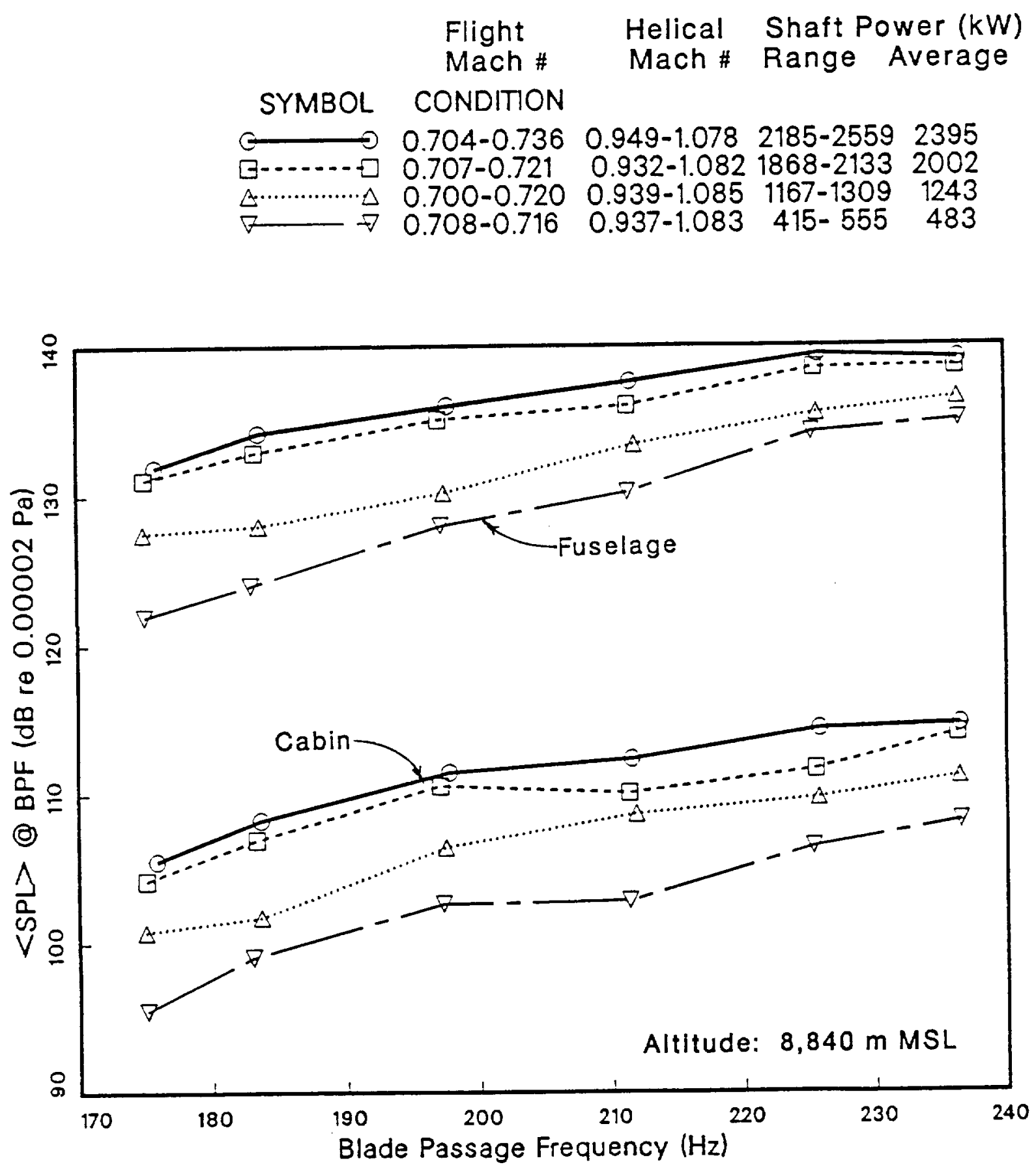

FIGURE 58: Fuselage and Cabin $\angle S P L>S$ versus BPF and Four Average Shaft Powers at an Altitude of $8,840 \mathrm{~m}$. 

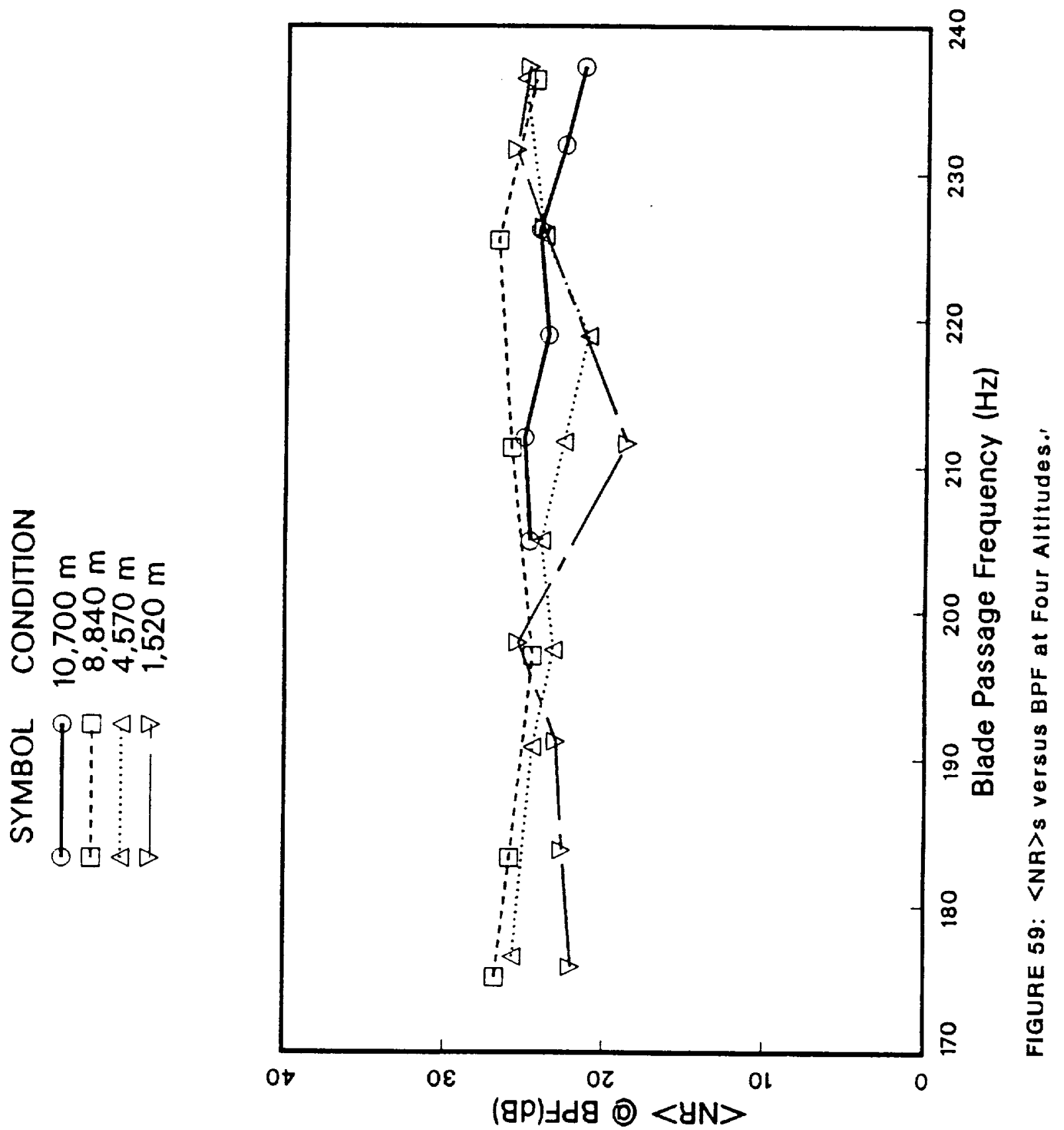

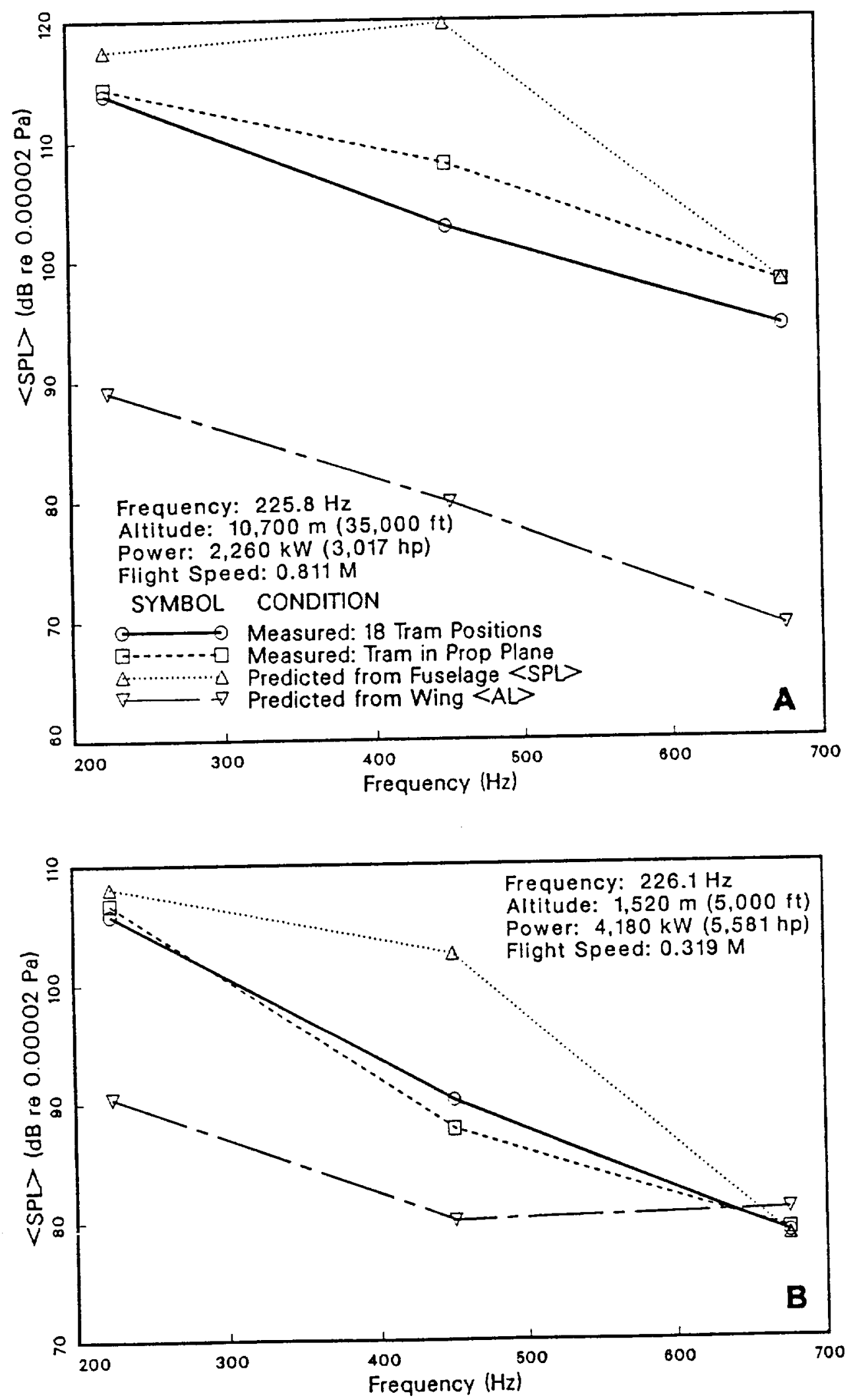

FIGURE 60: Cabin <SPL>s for the First Three BPF Harmonics Measured at Two Altitudes and Predicted from the Ground/Fllght WIng Acceleration and Fuselage SPL Analyses. 


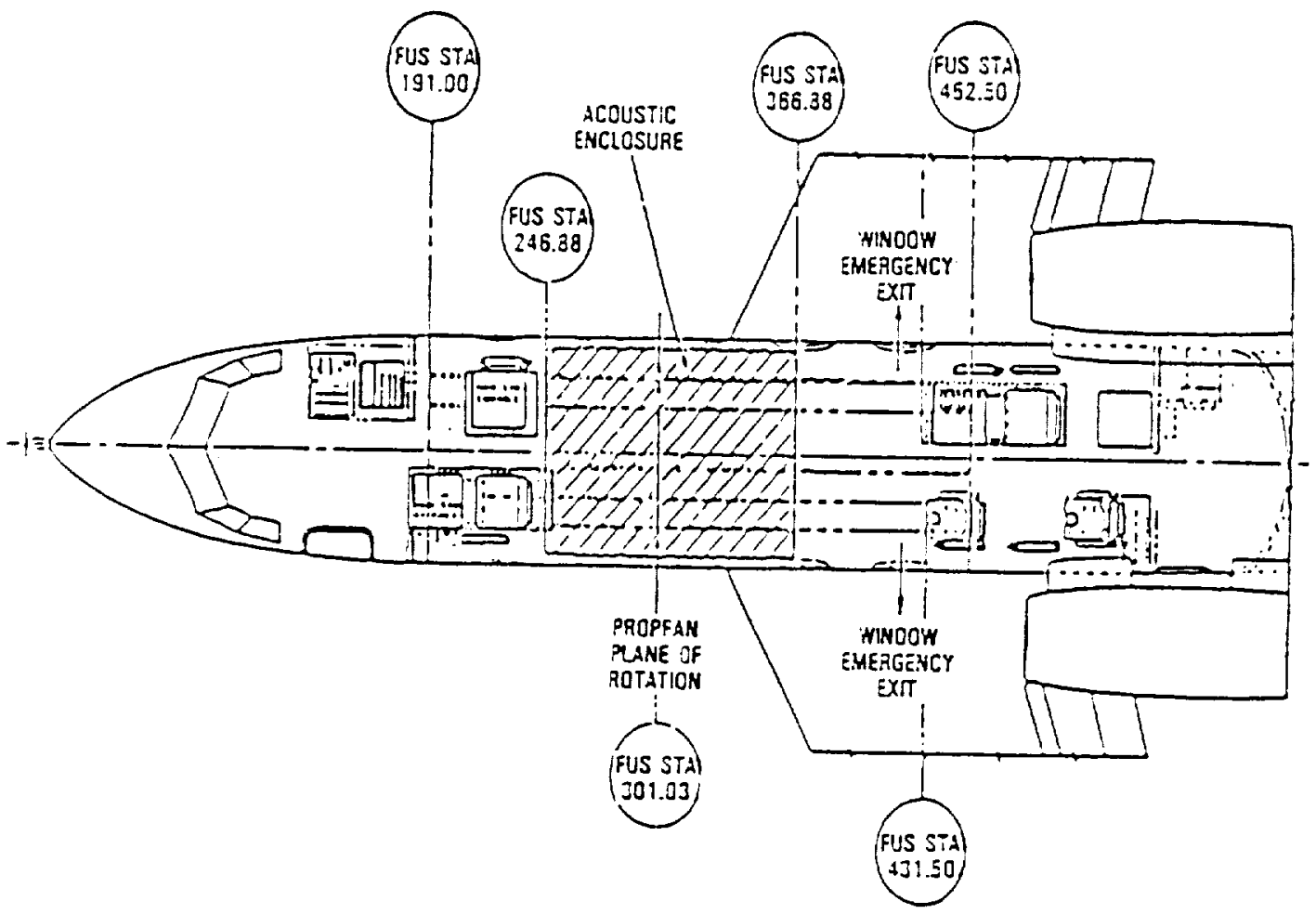

FIGURE 61: Cabin Interior Arrangement with Acoustic Treatment.

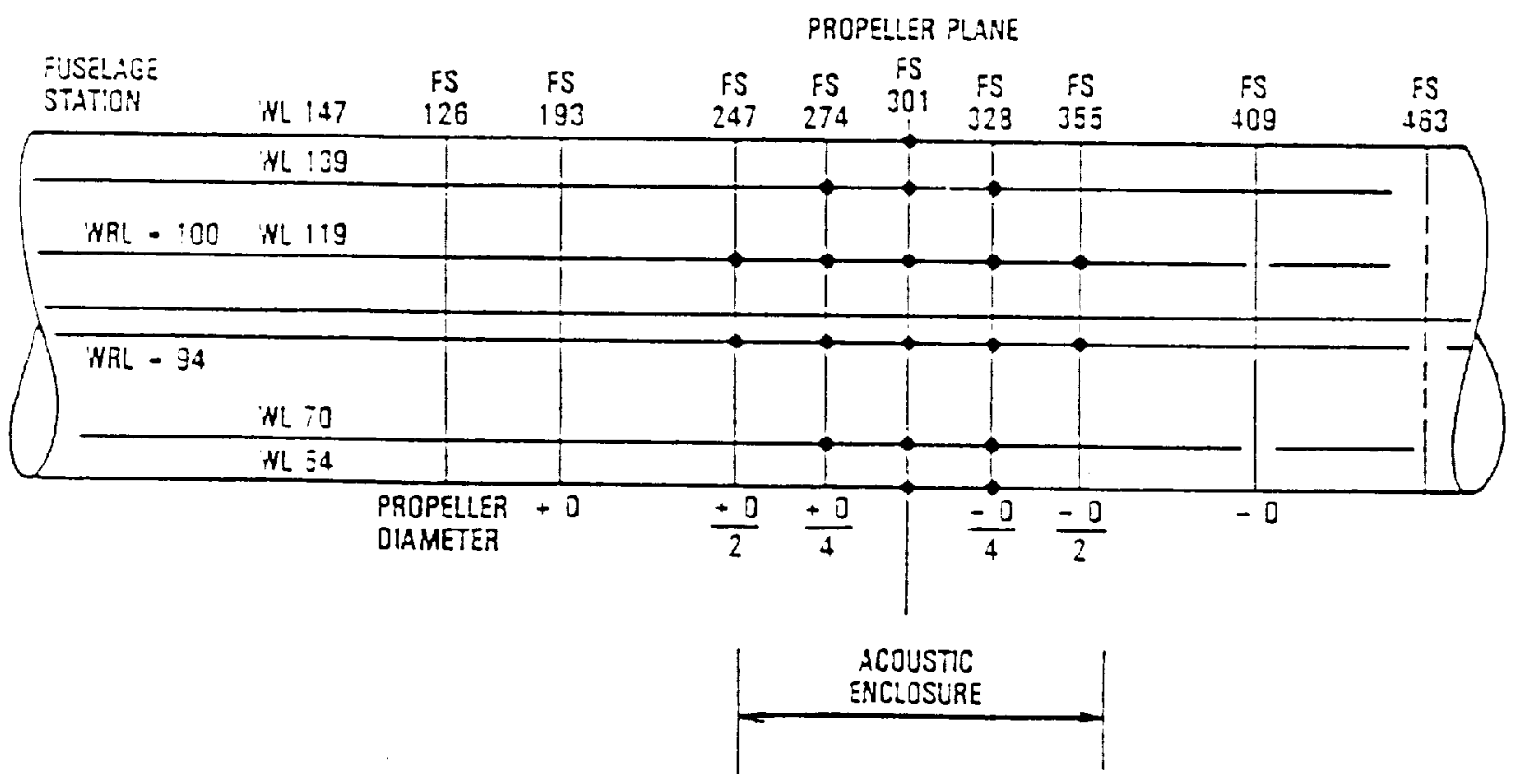

FIGURE 62: Fuselage Surface Microphone Locations. 


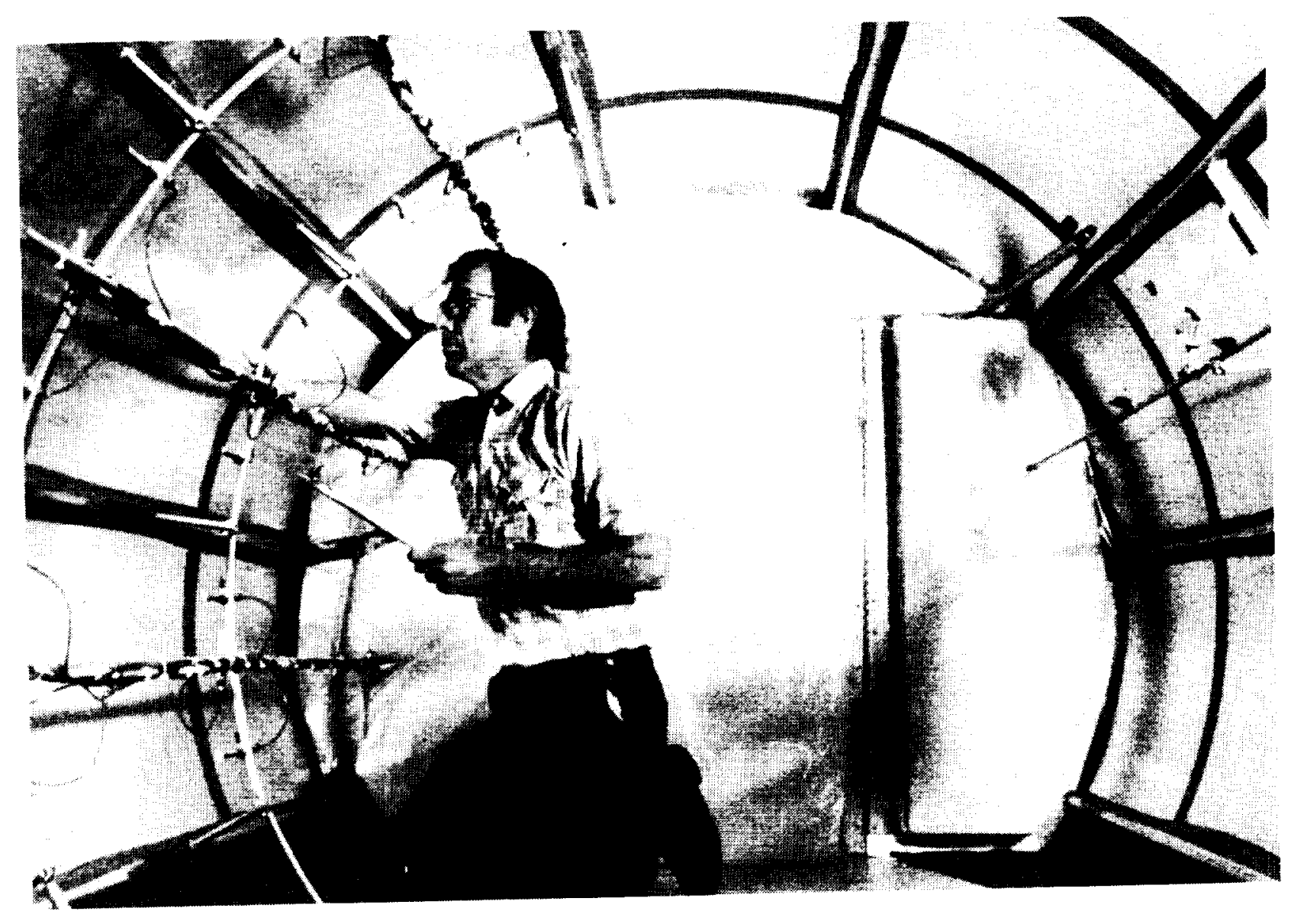

- MICROPHONES

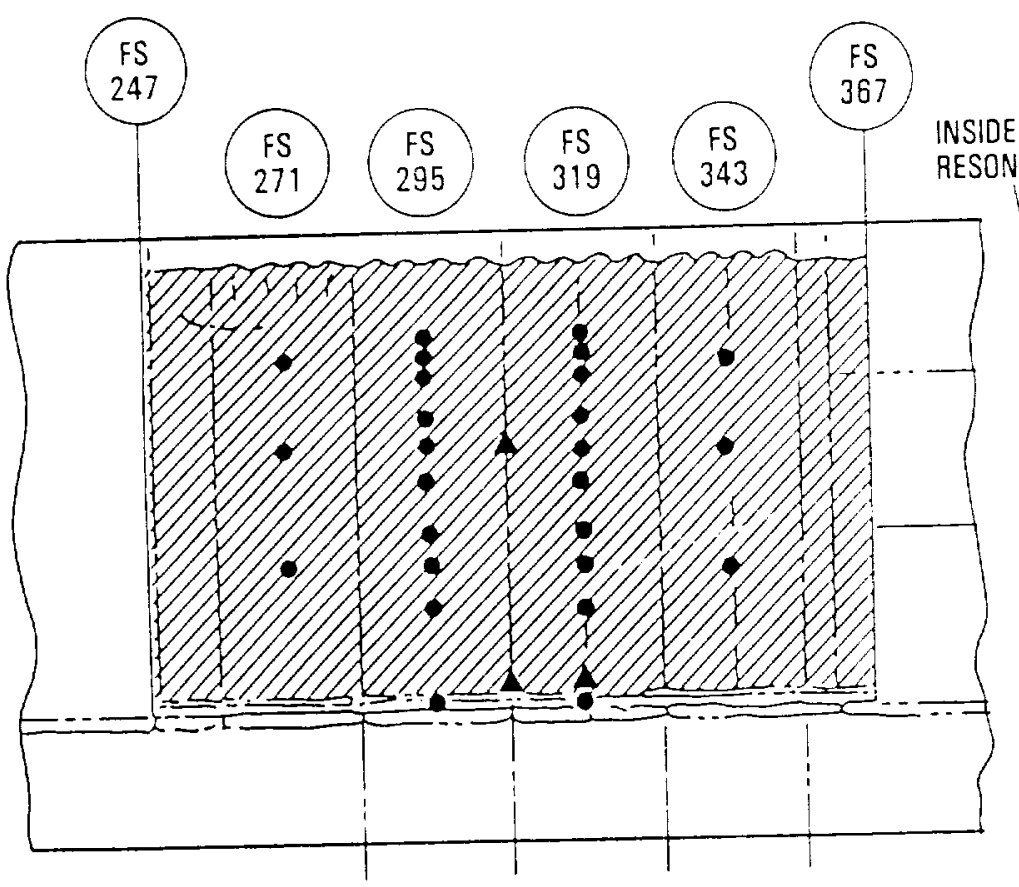

LH SIDE VIEW

(25) INSIDE ENCLOSURE

(3) BETWEEN WALLS

(1) INSIDE RESONATOR

(2) BELOW FLOOR

- ACCELEROMETERS

(2) TRIM

(2) FLOOR

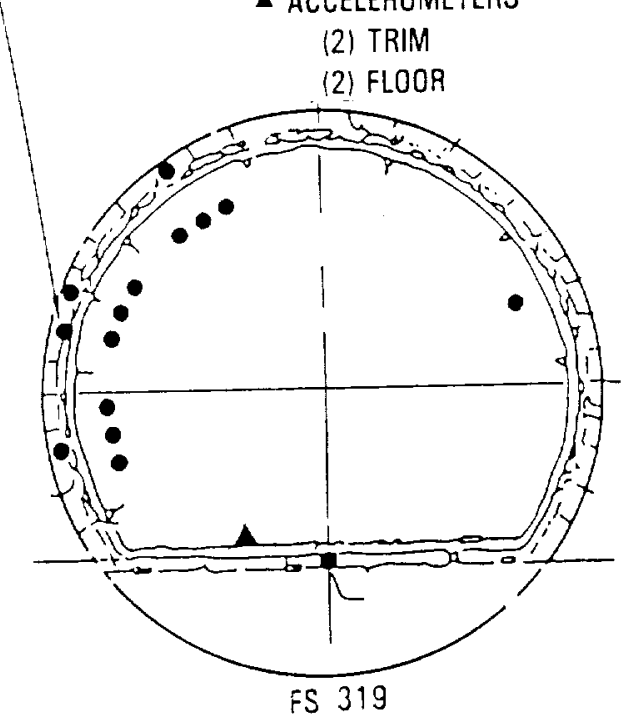

FIGURE 63: Interior Microphone and Accelerometer Locations. 


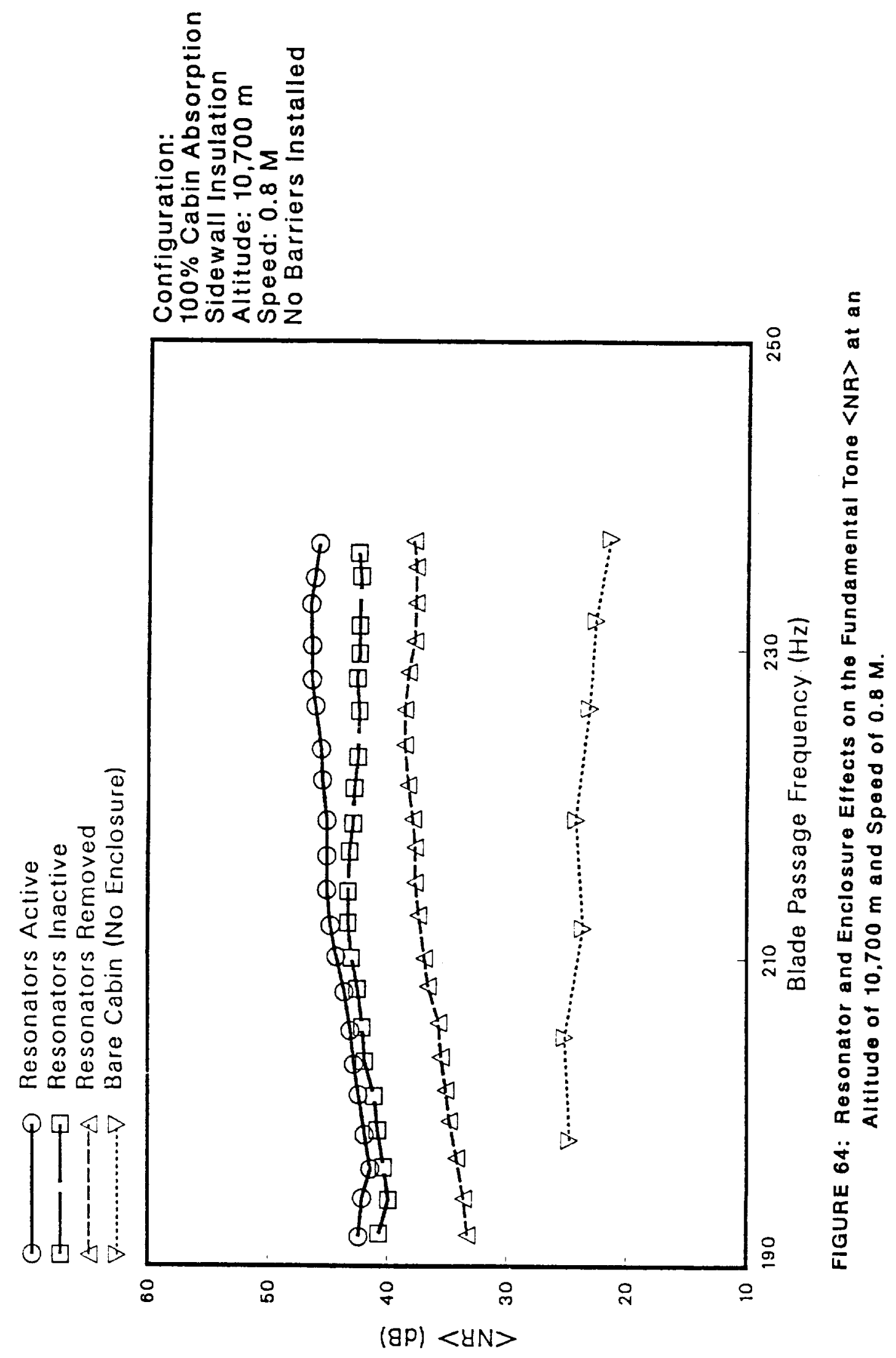




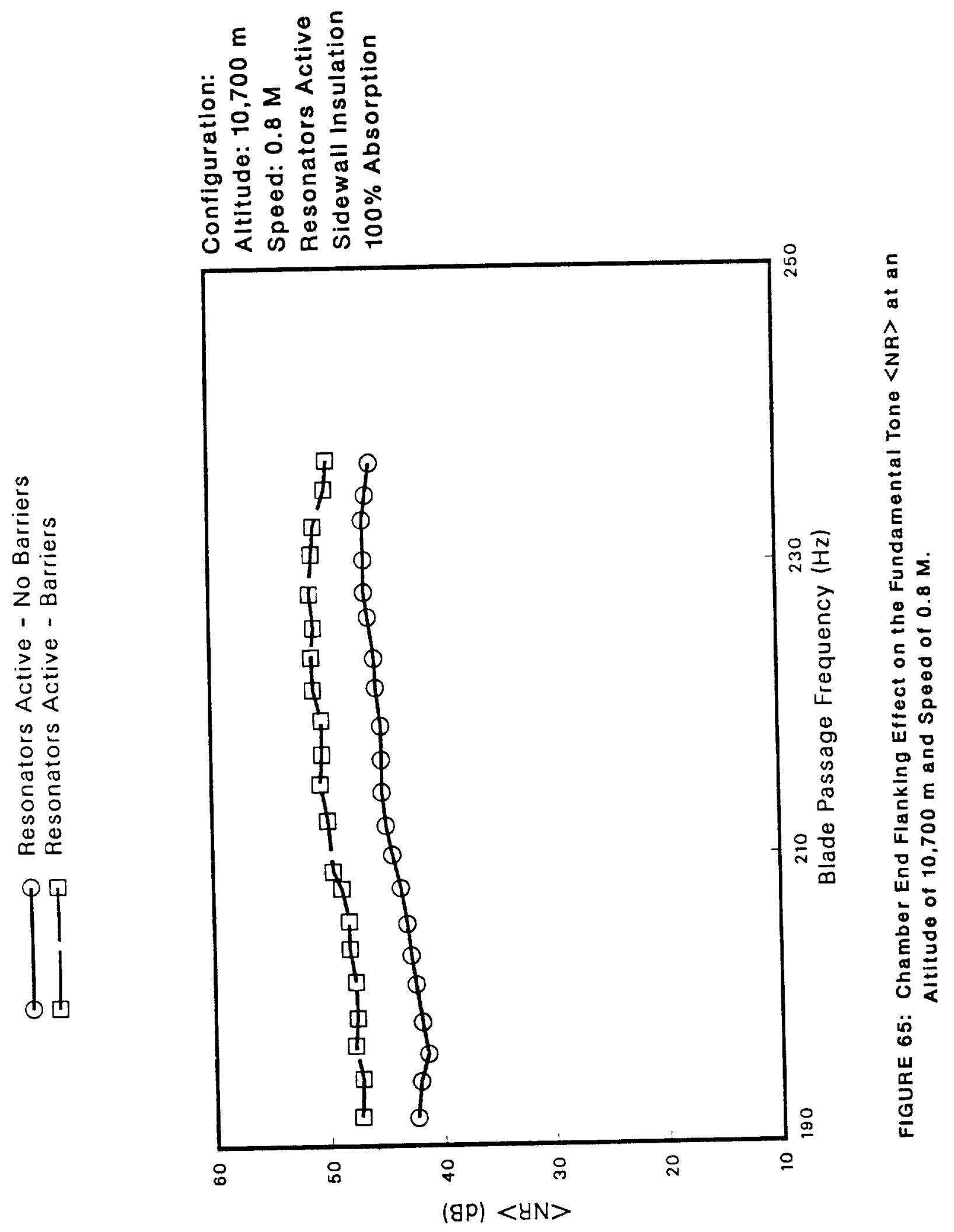




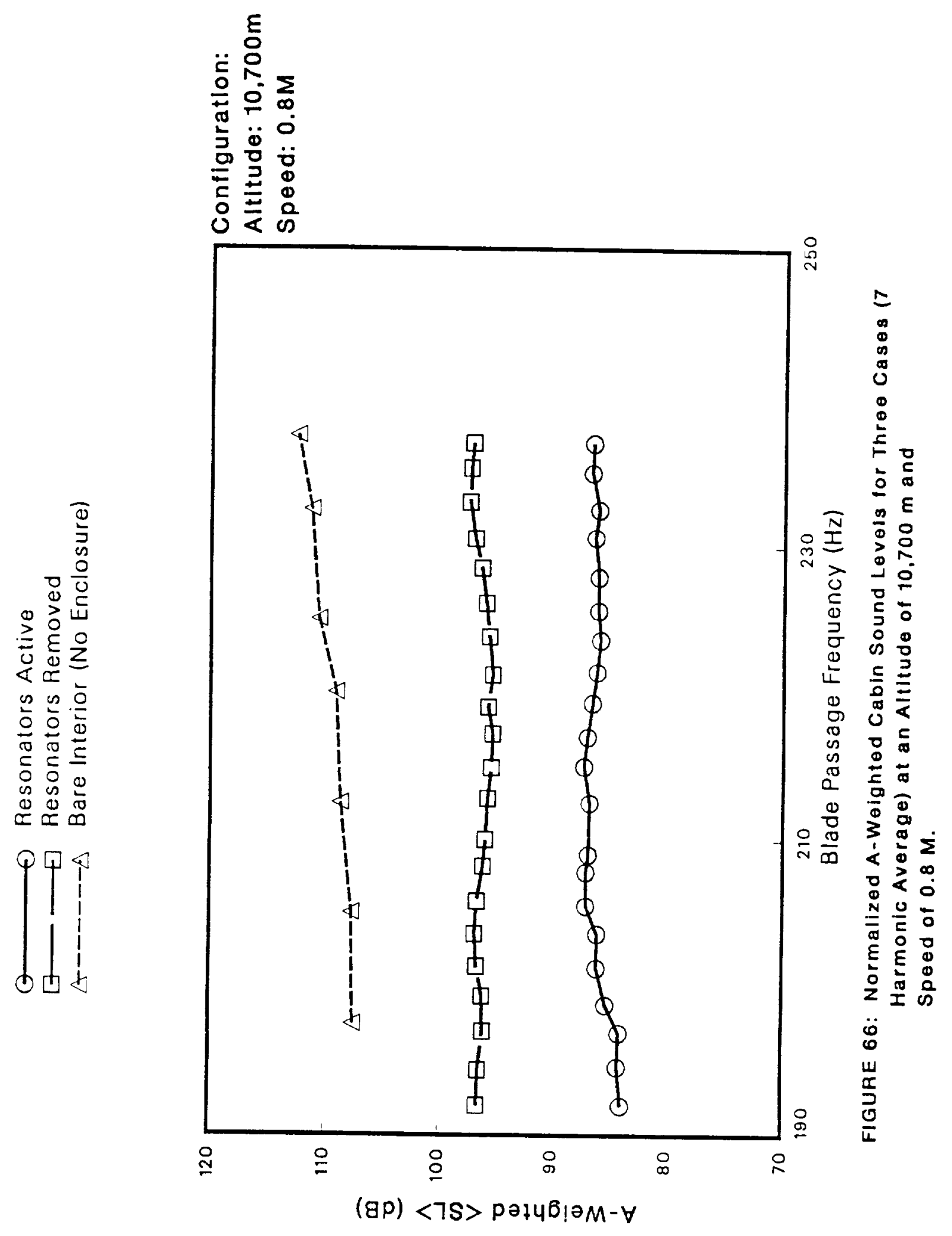




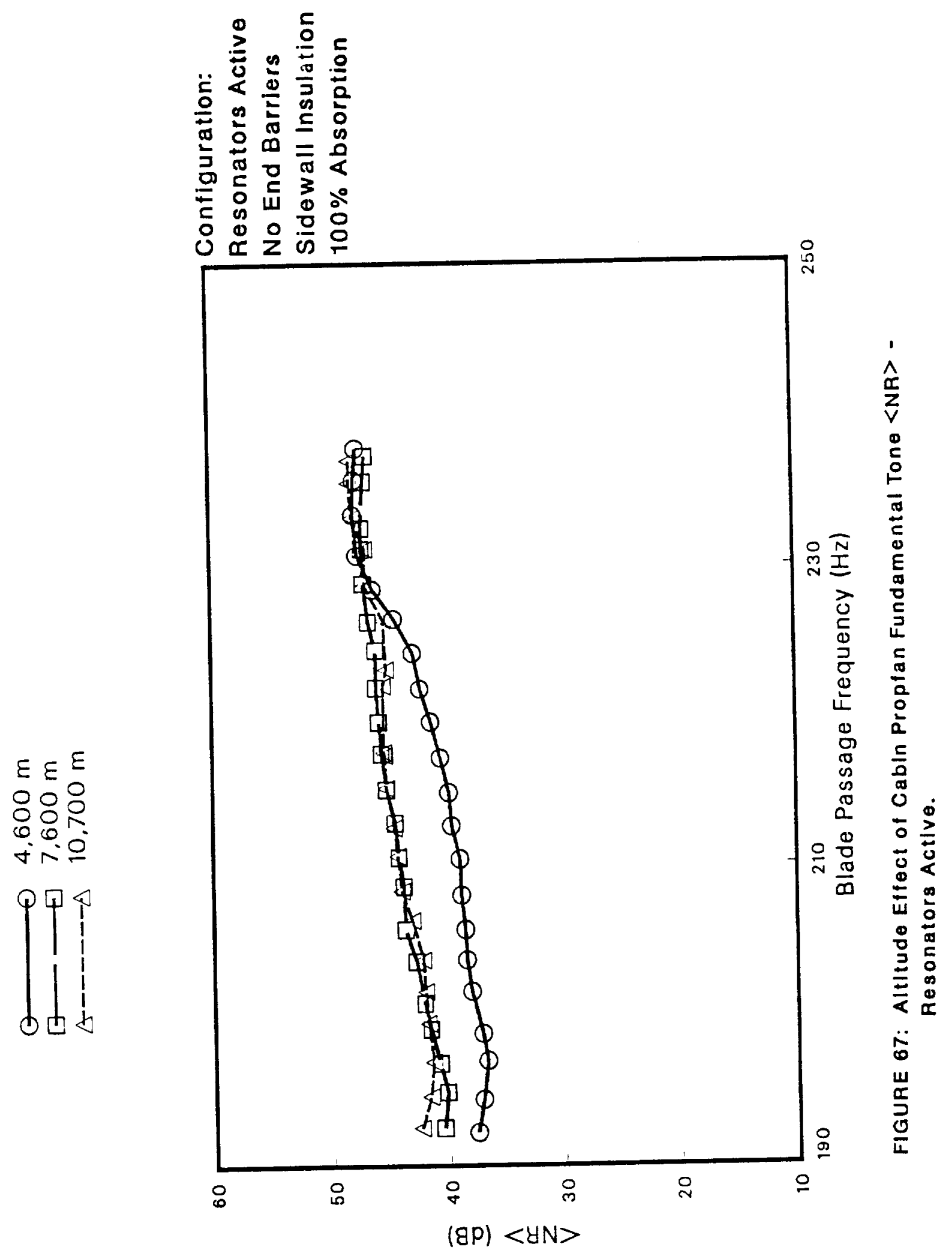




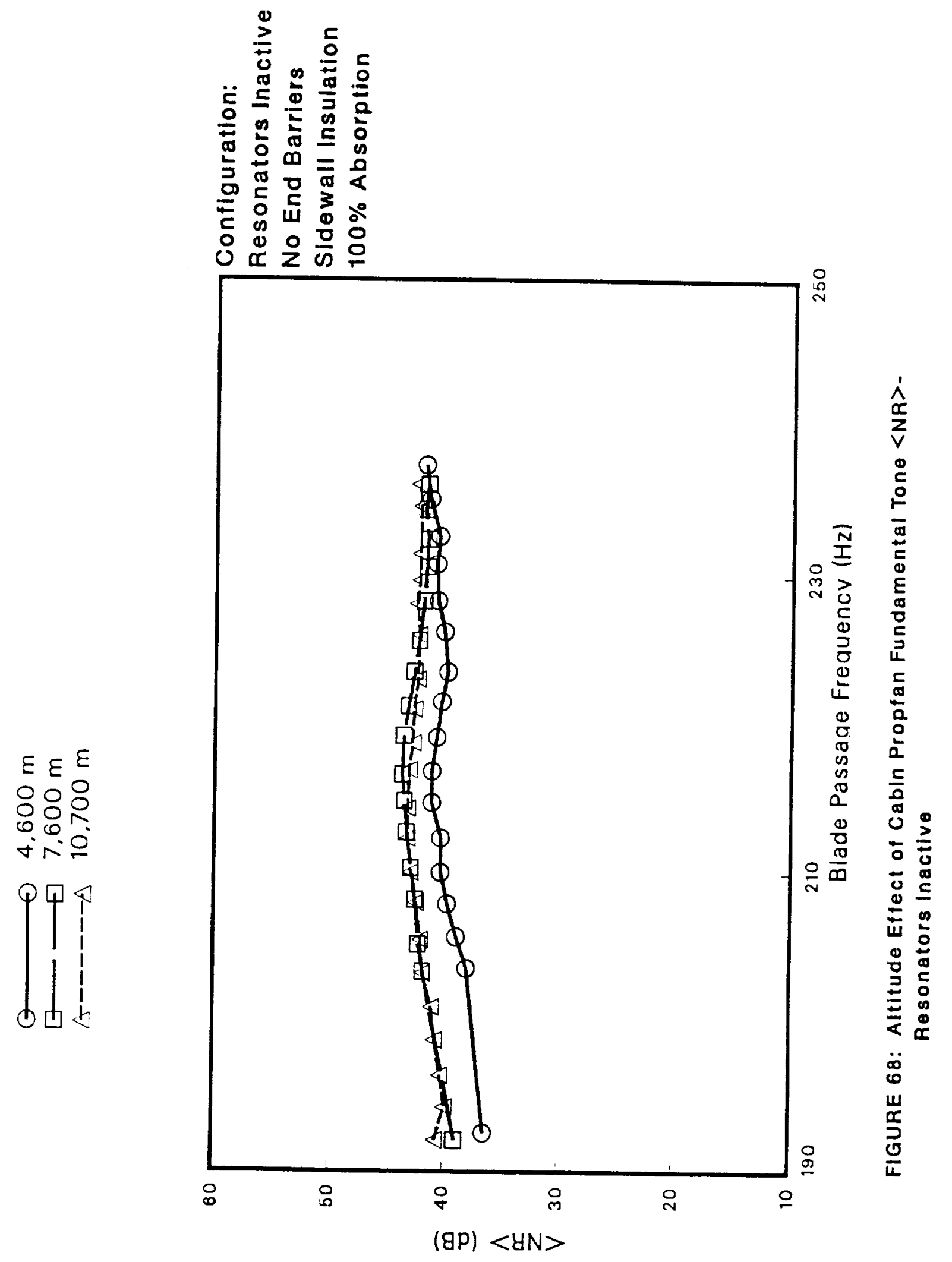




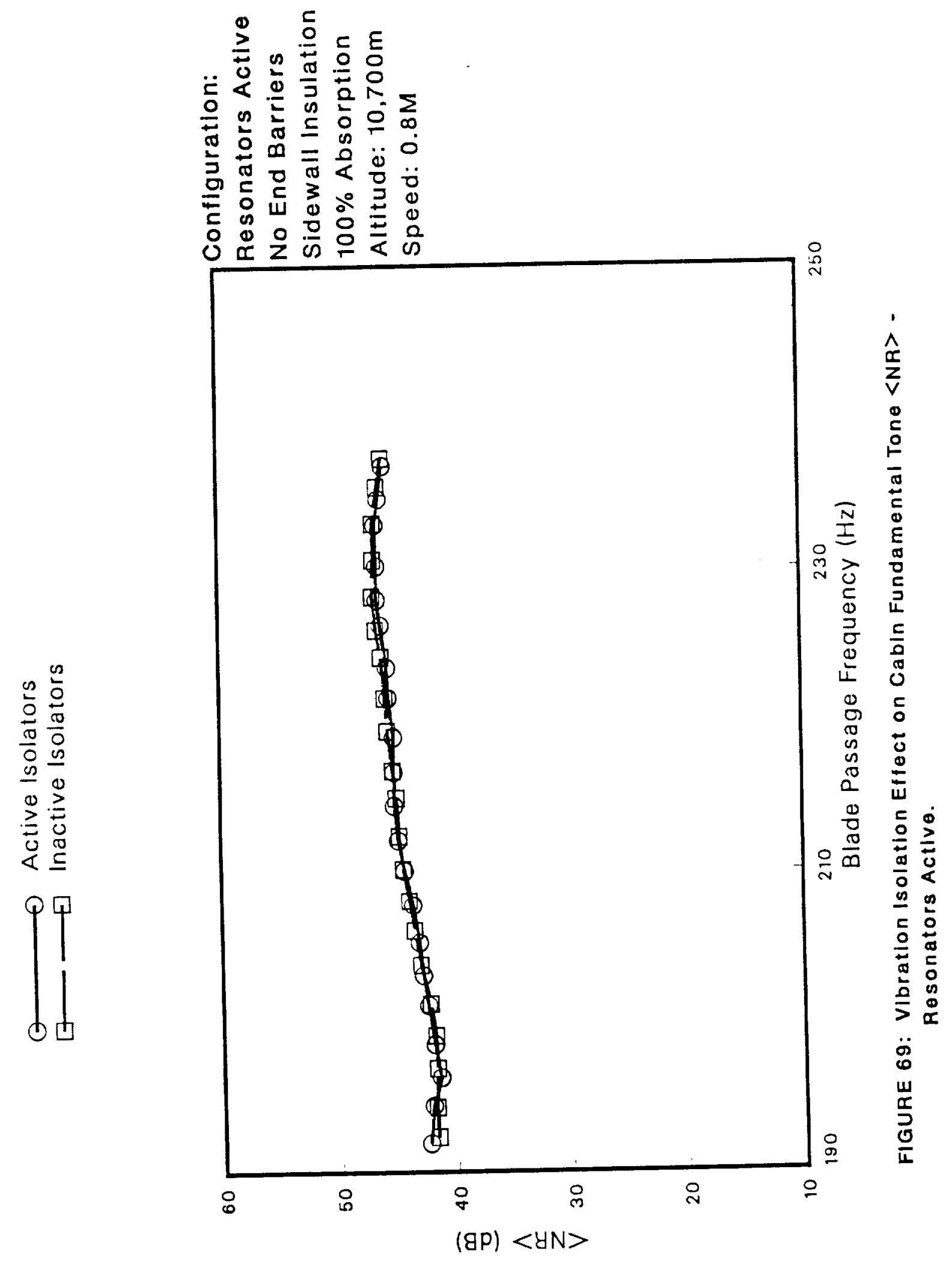




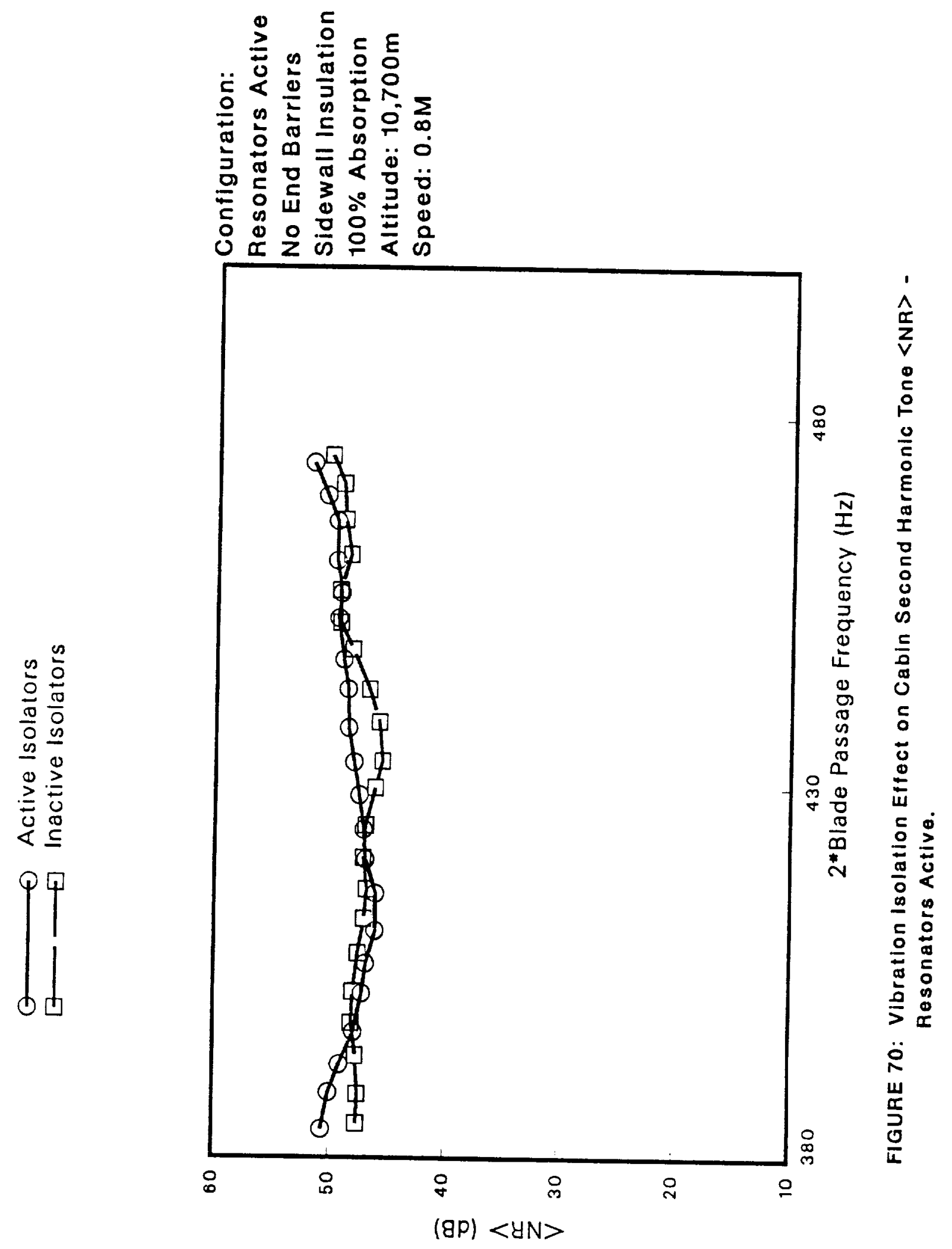




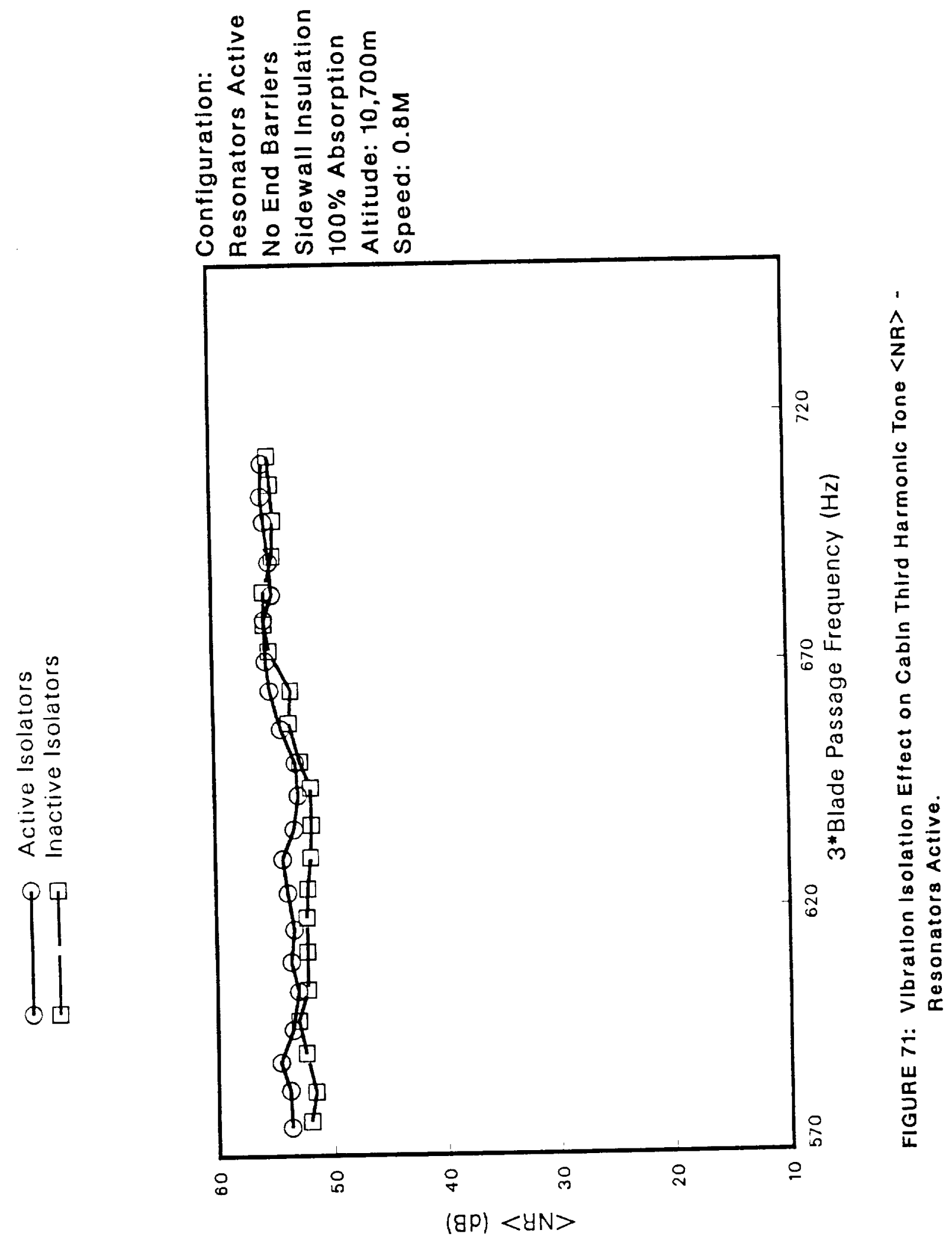




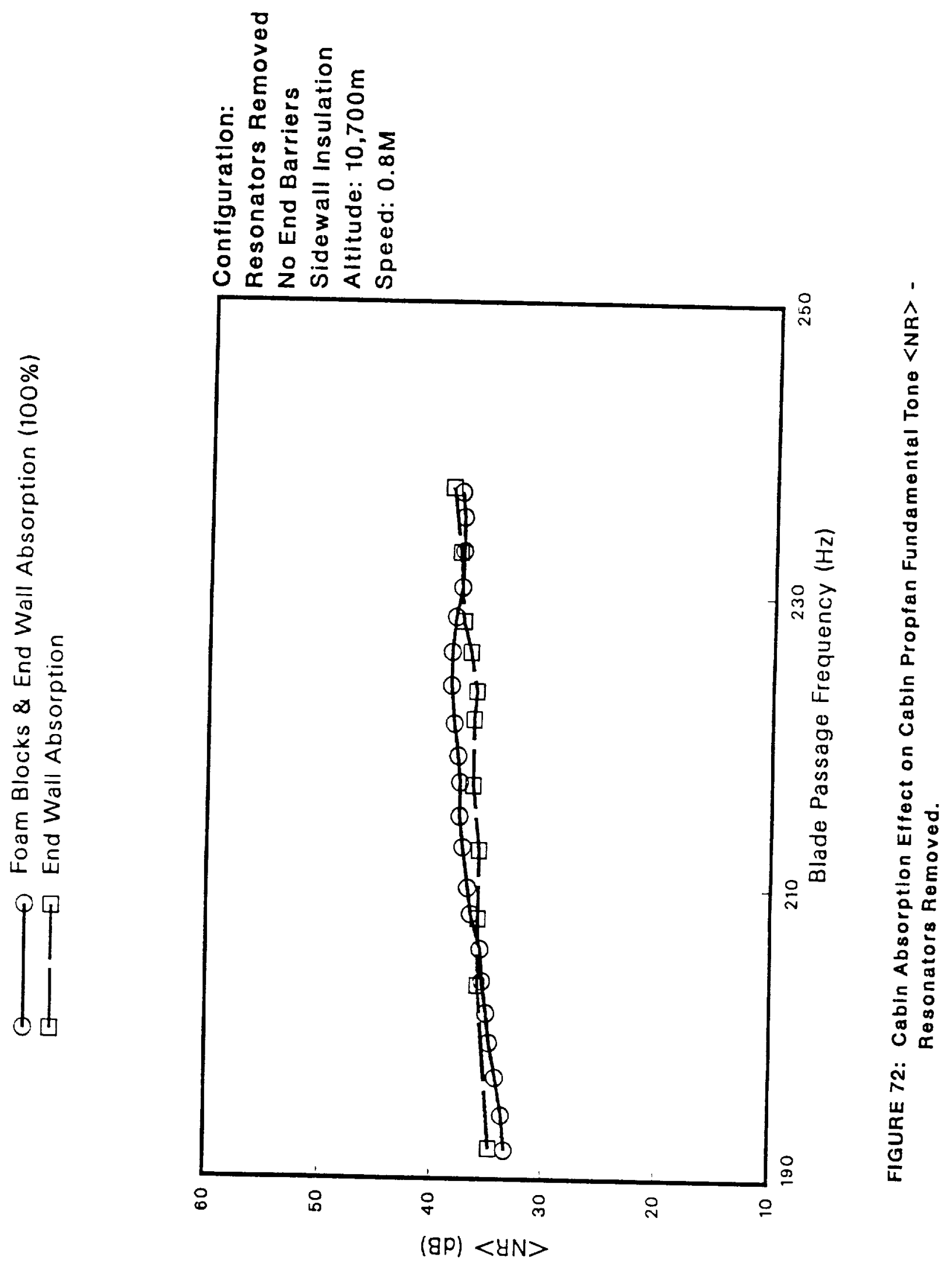




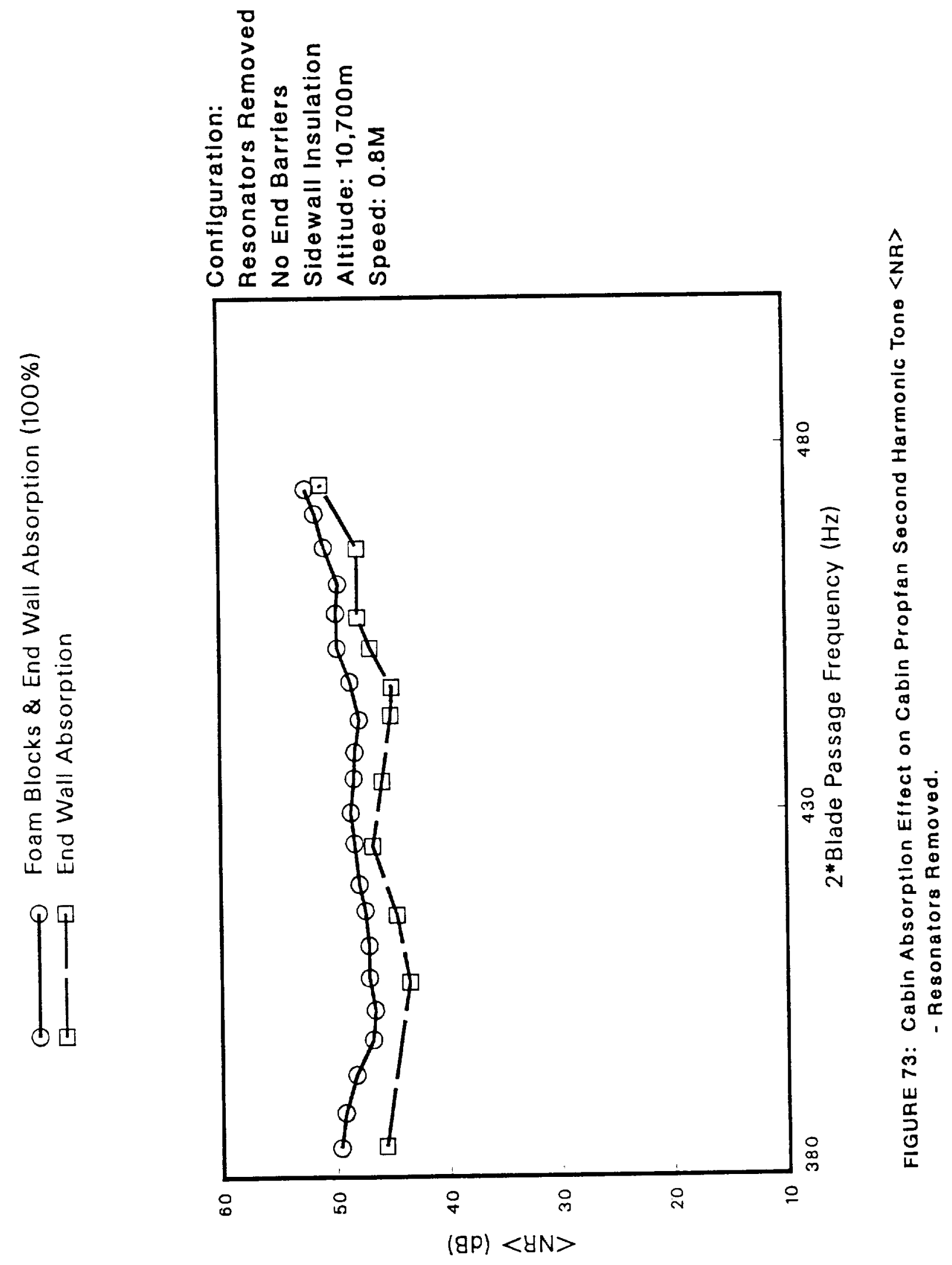




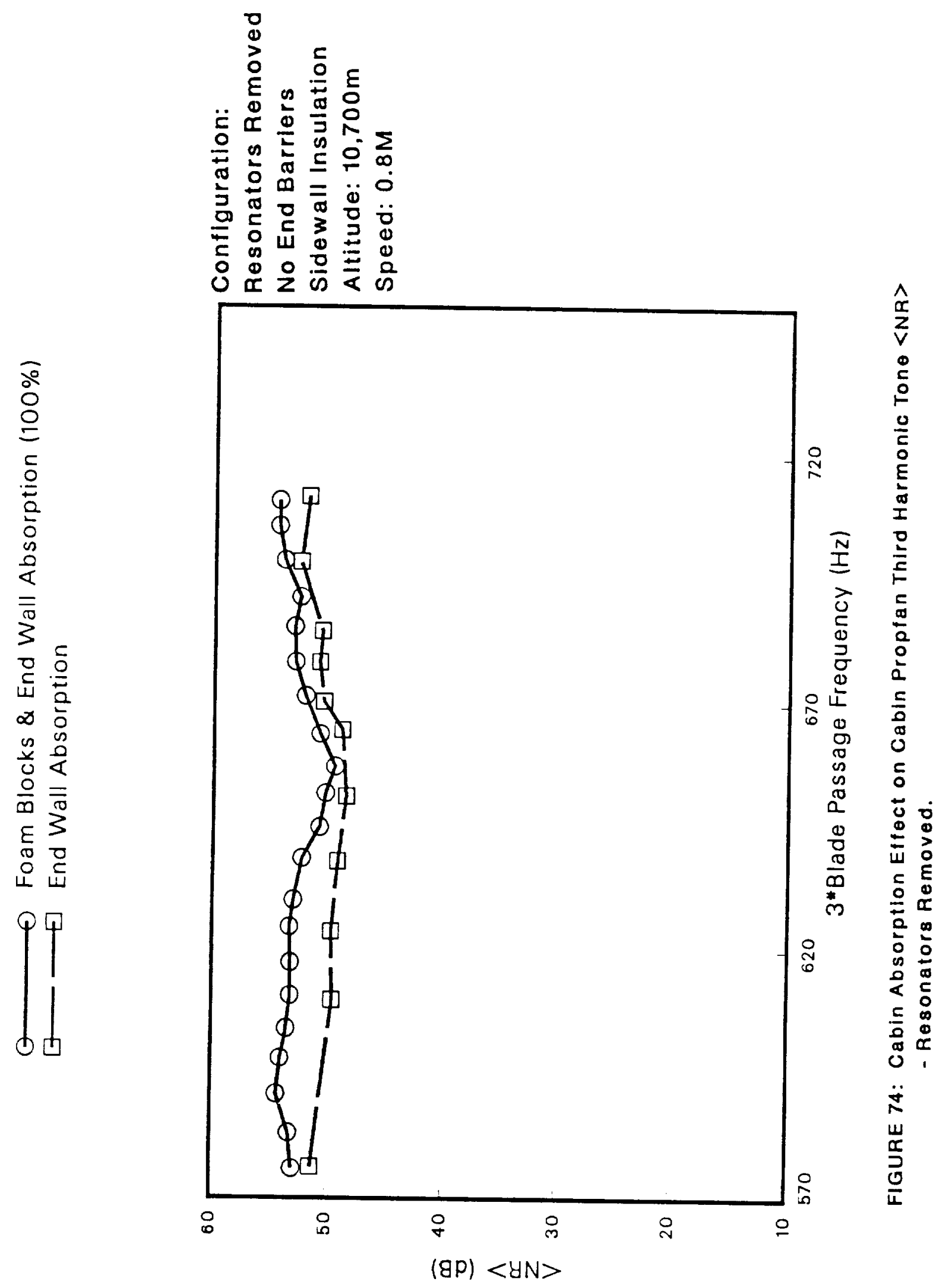




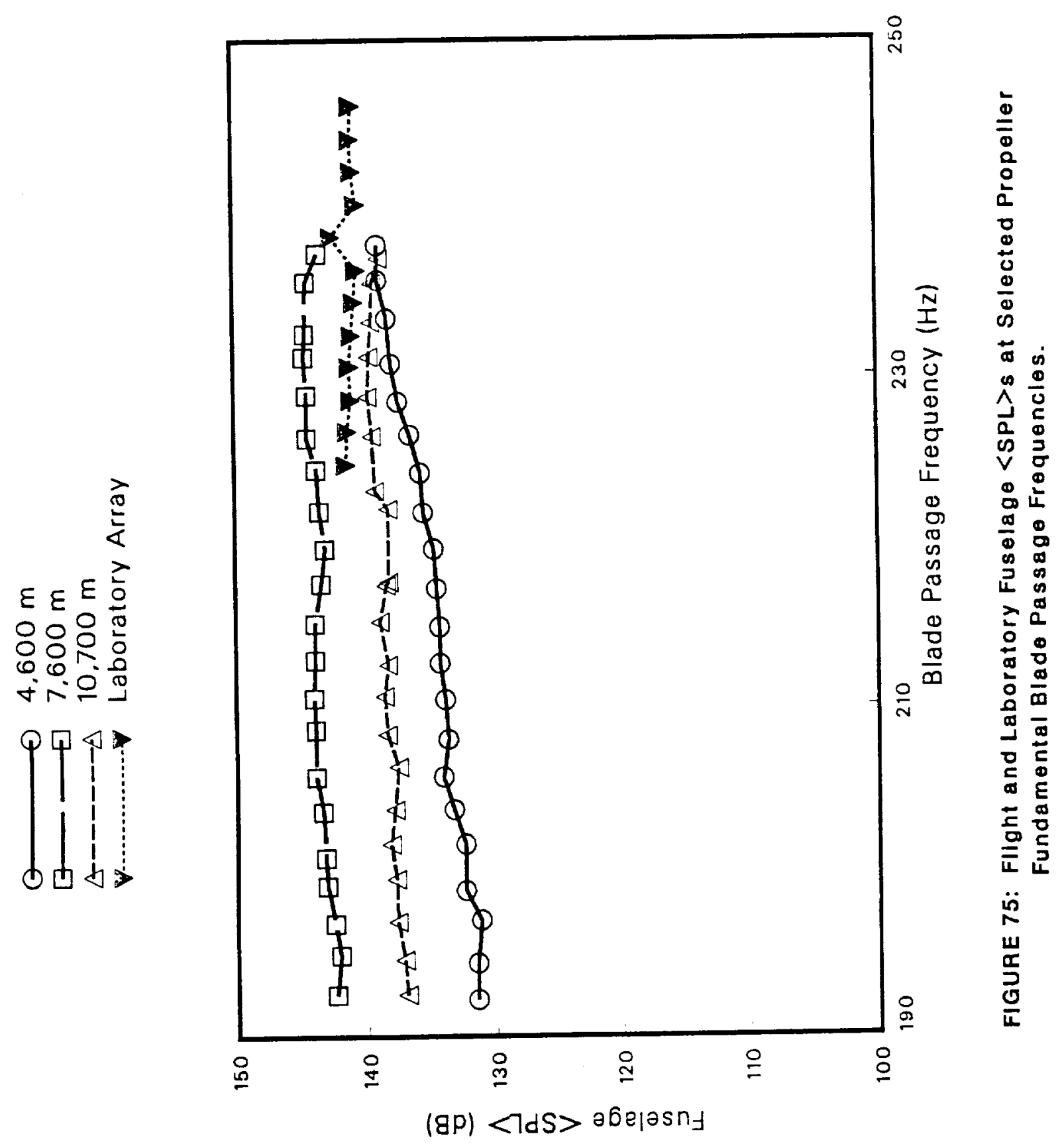




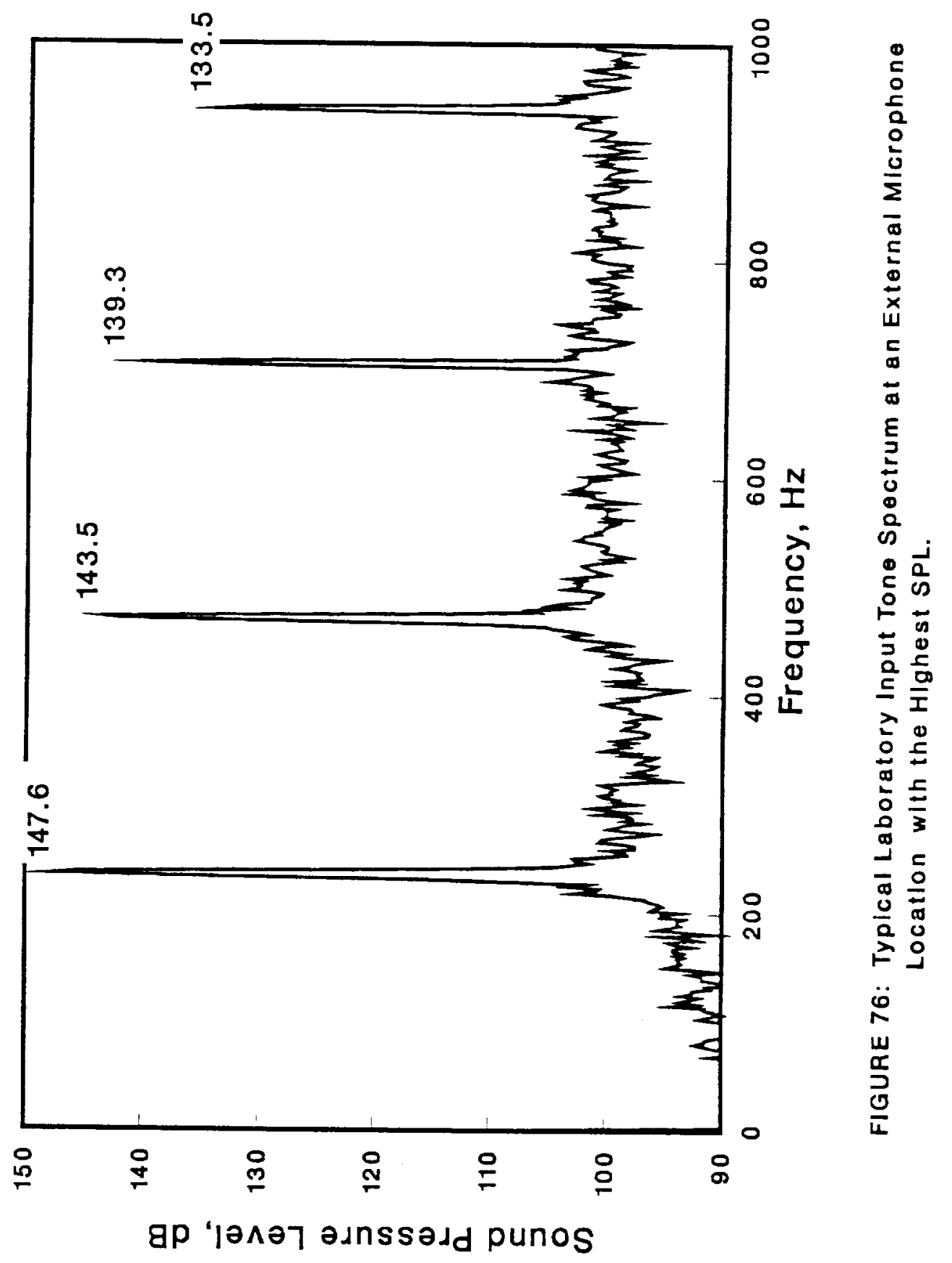




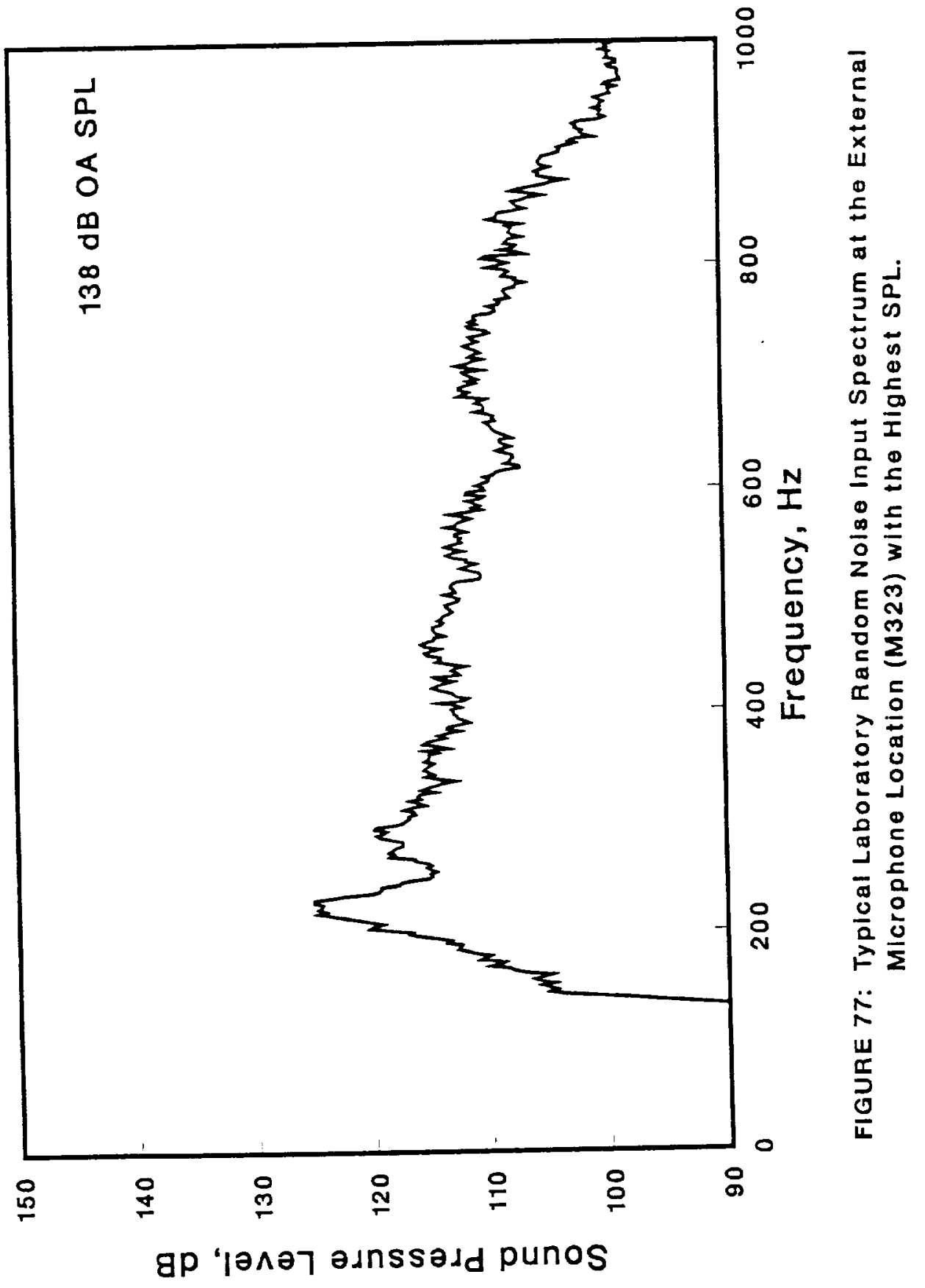




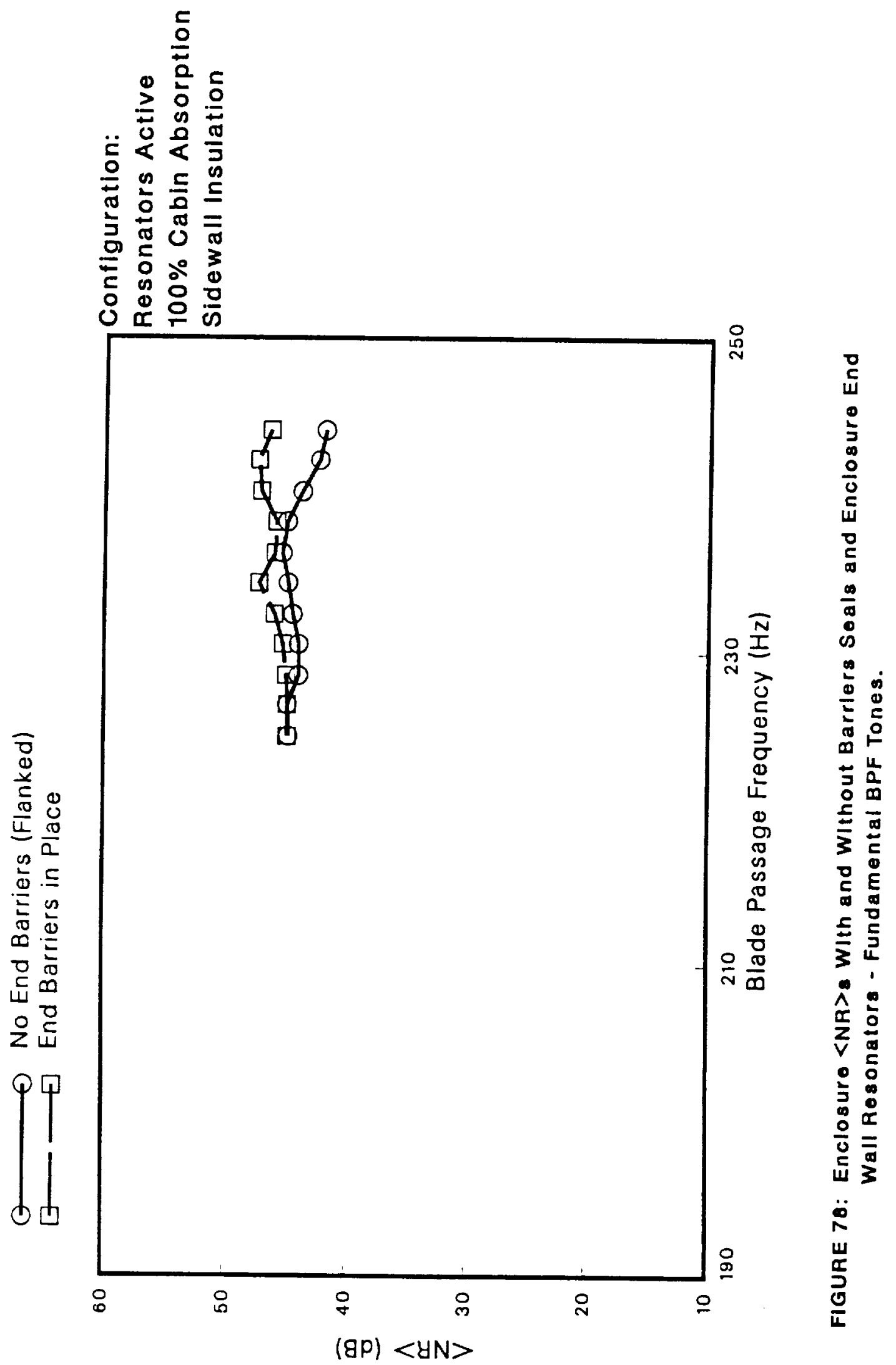




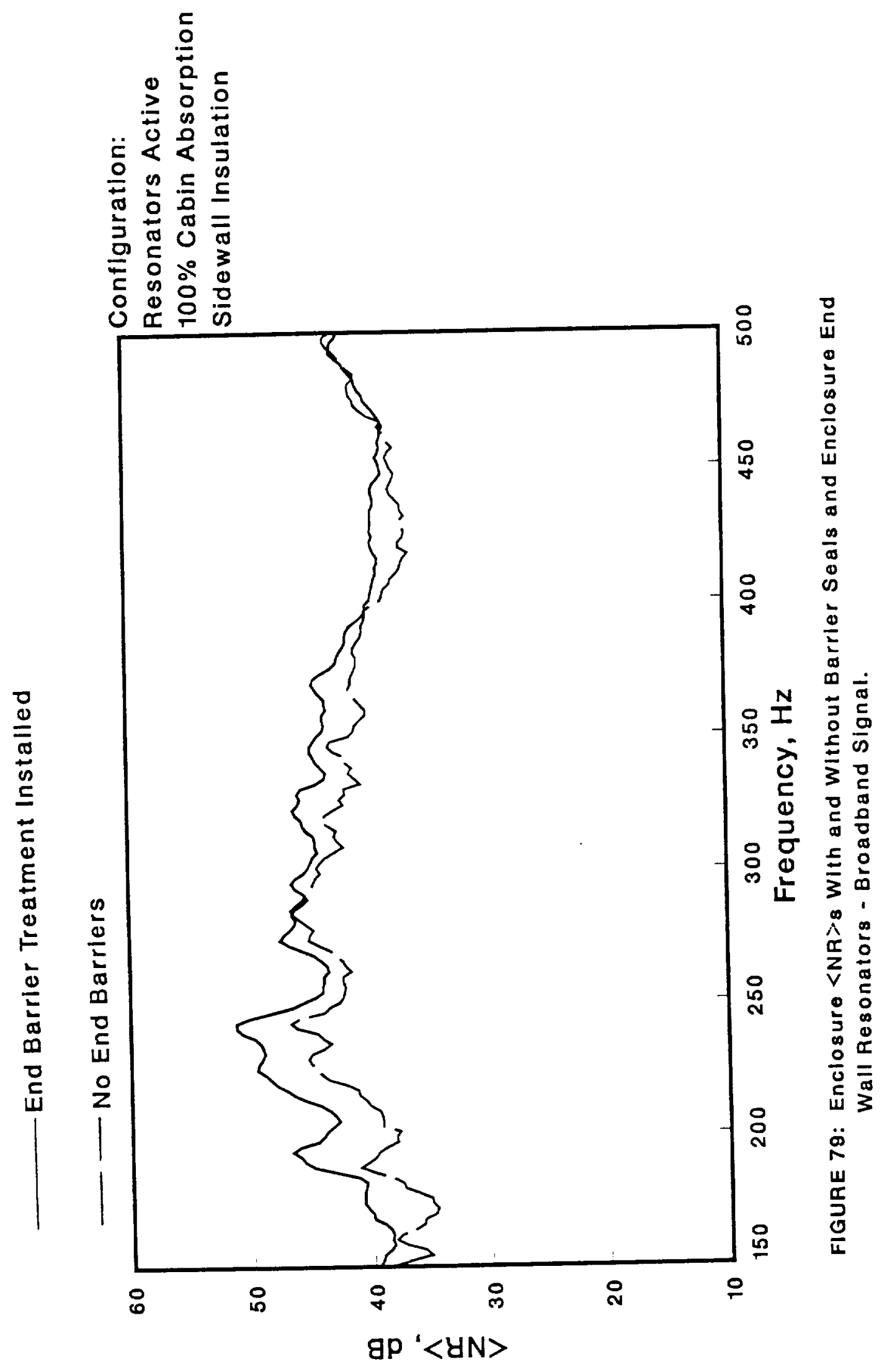




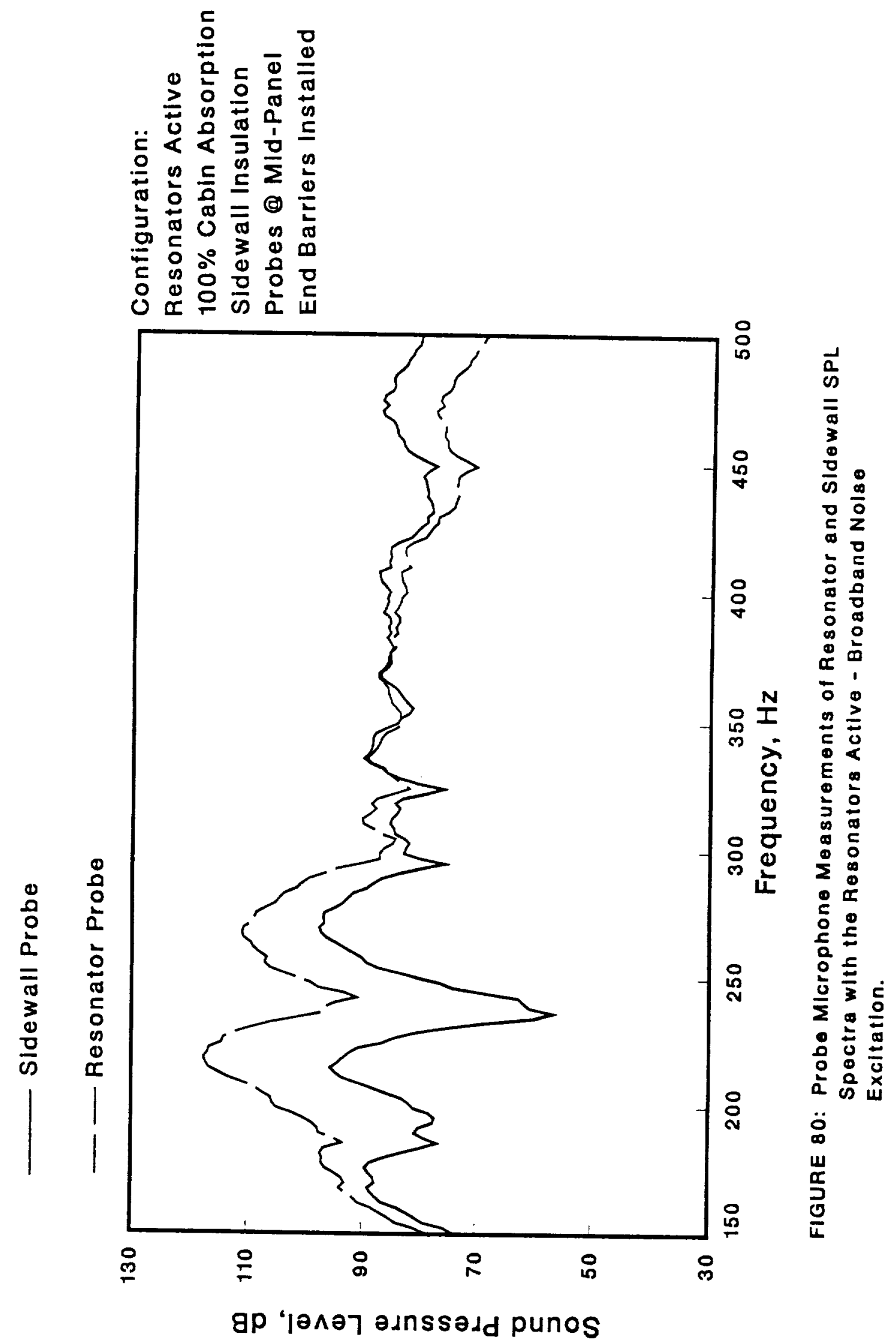




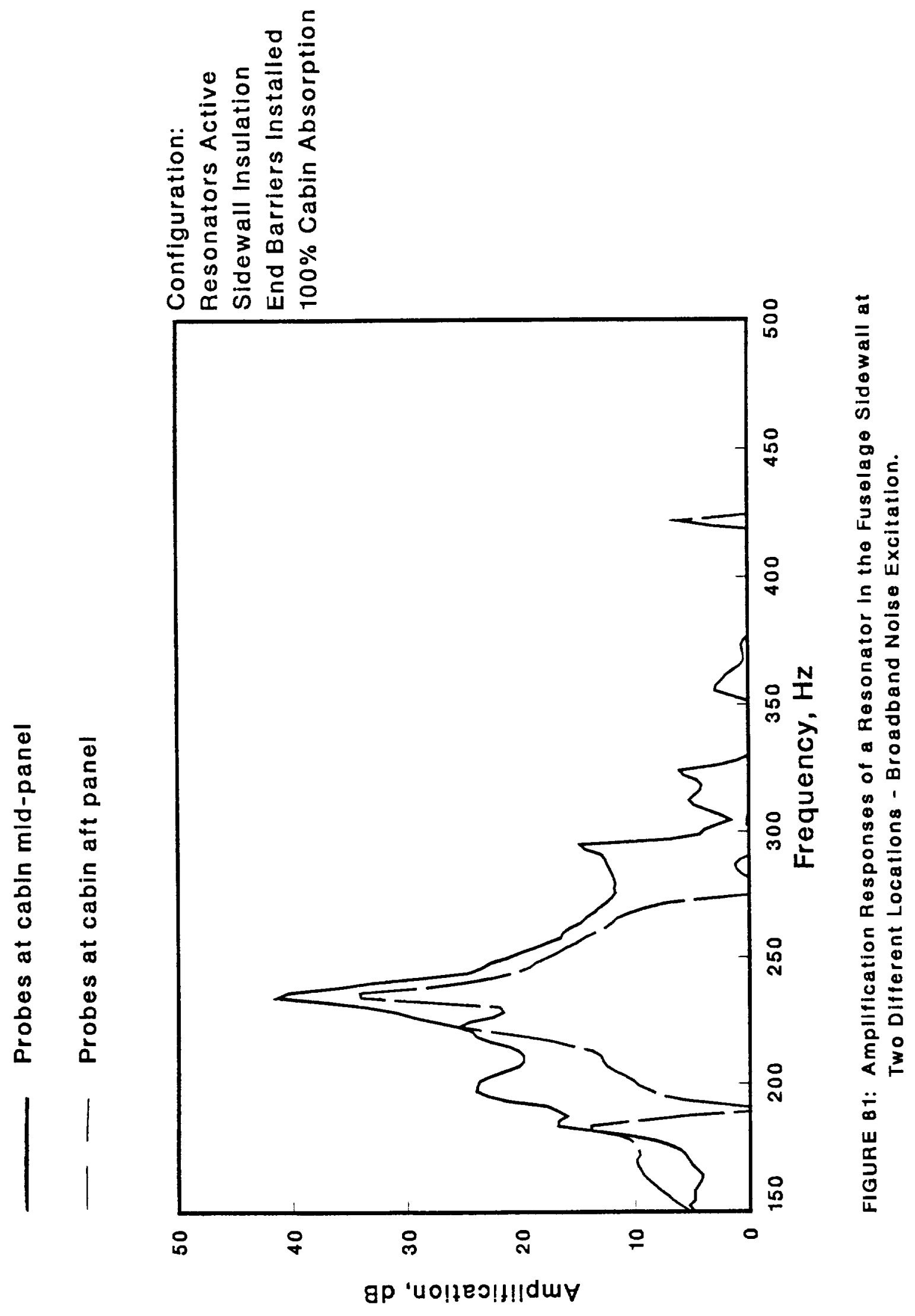




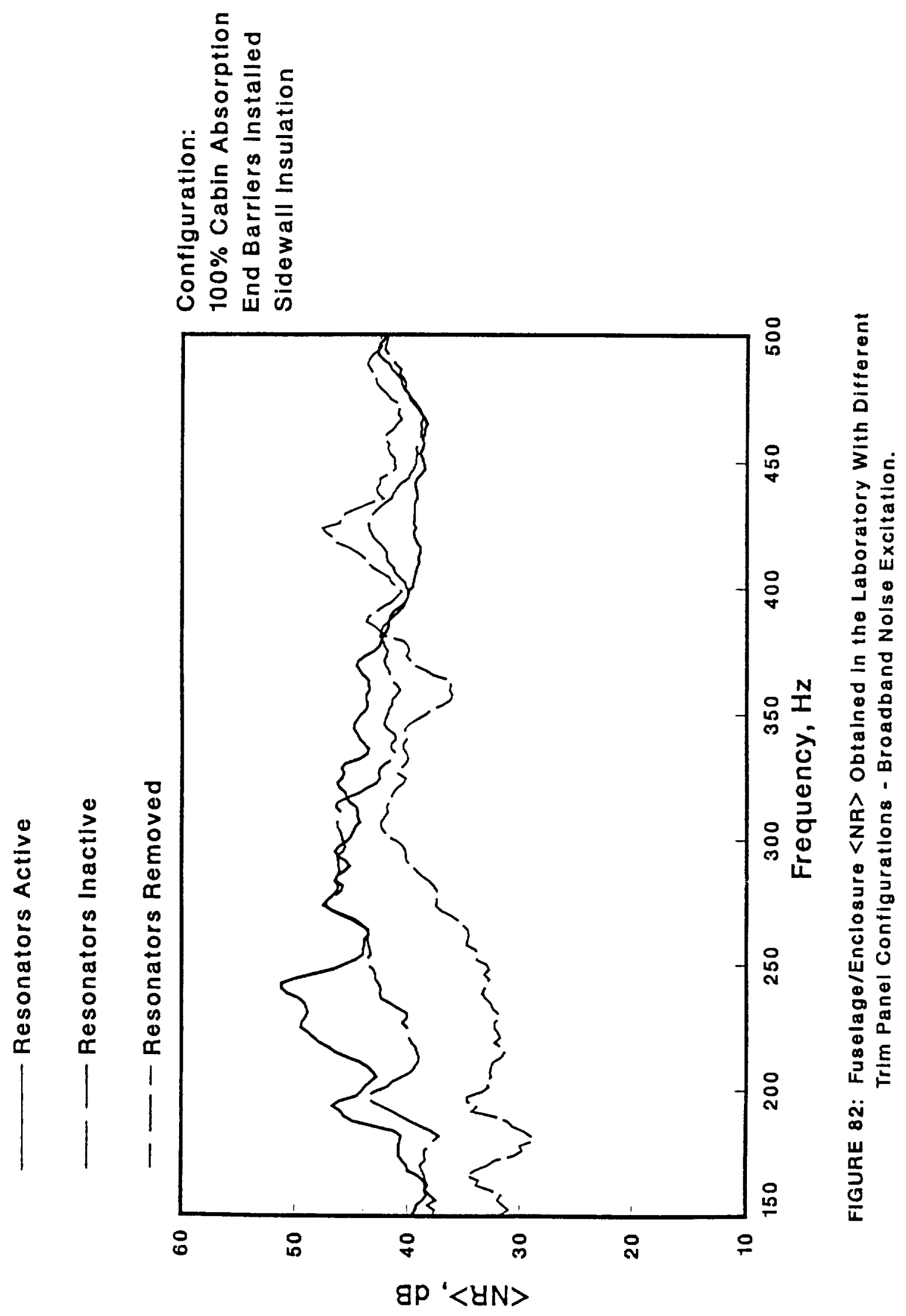




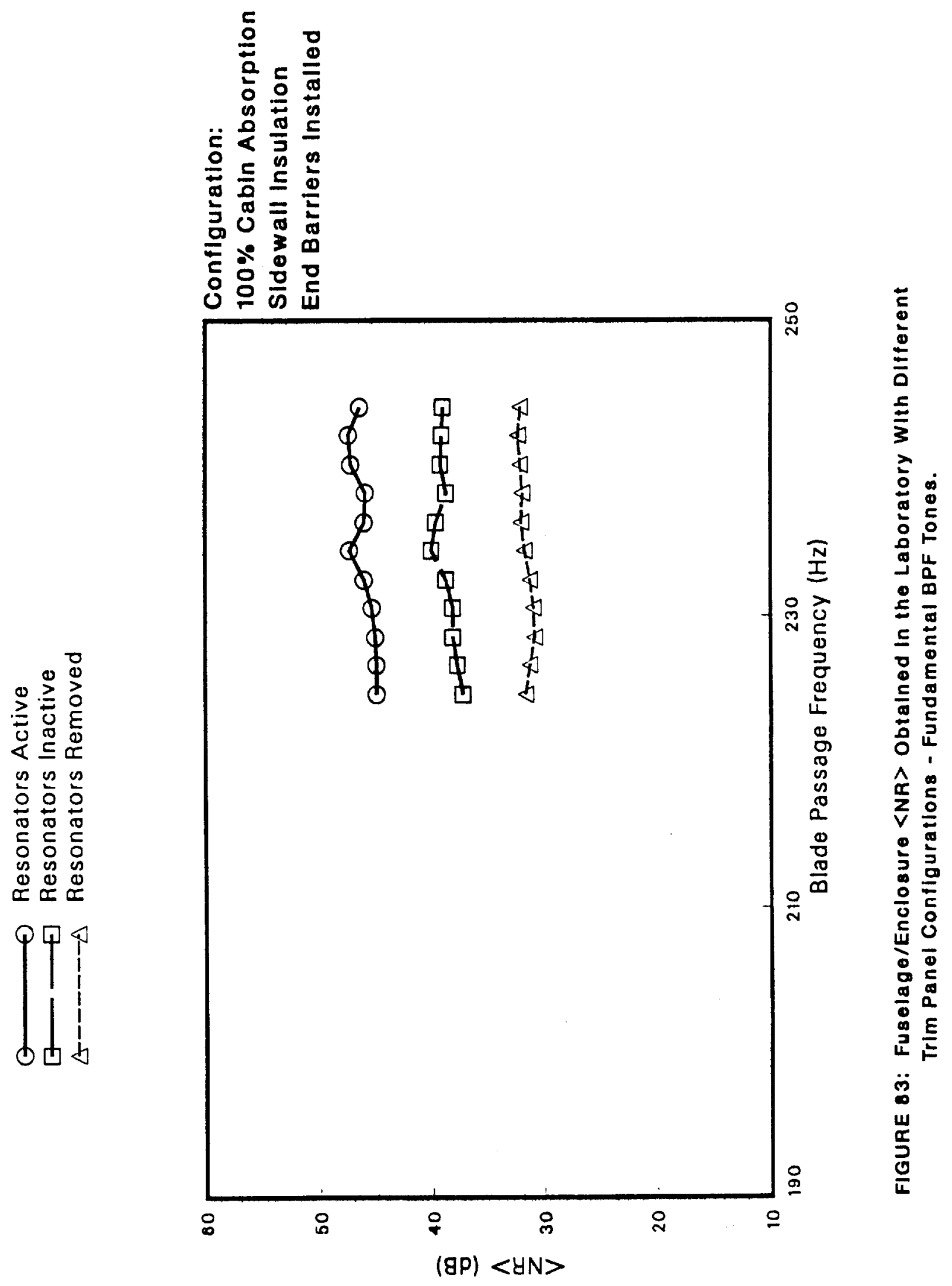




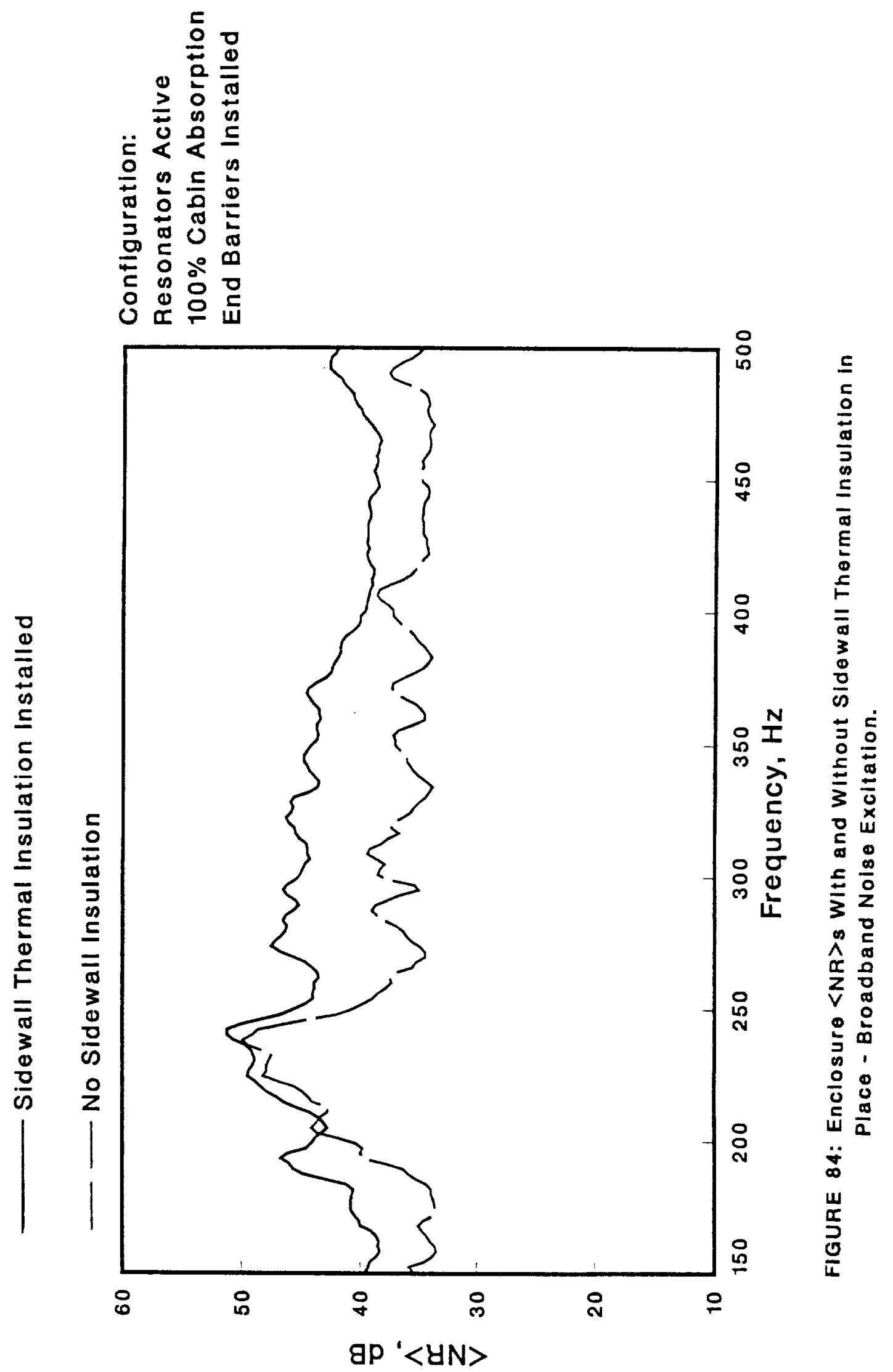




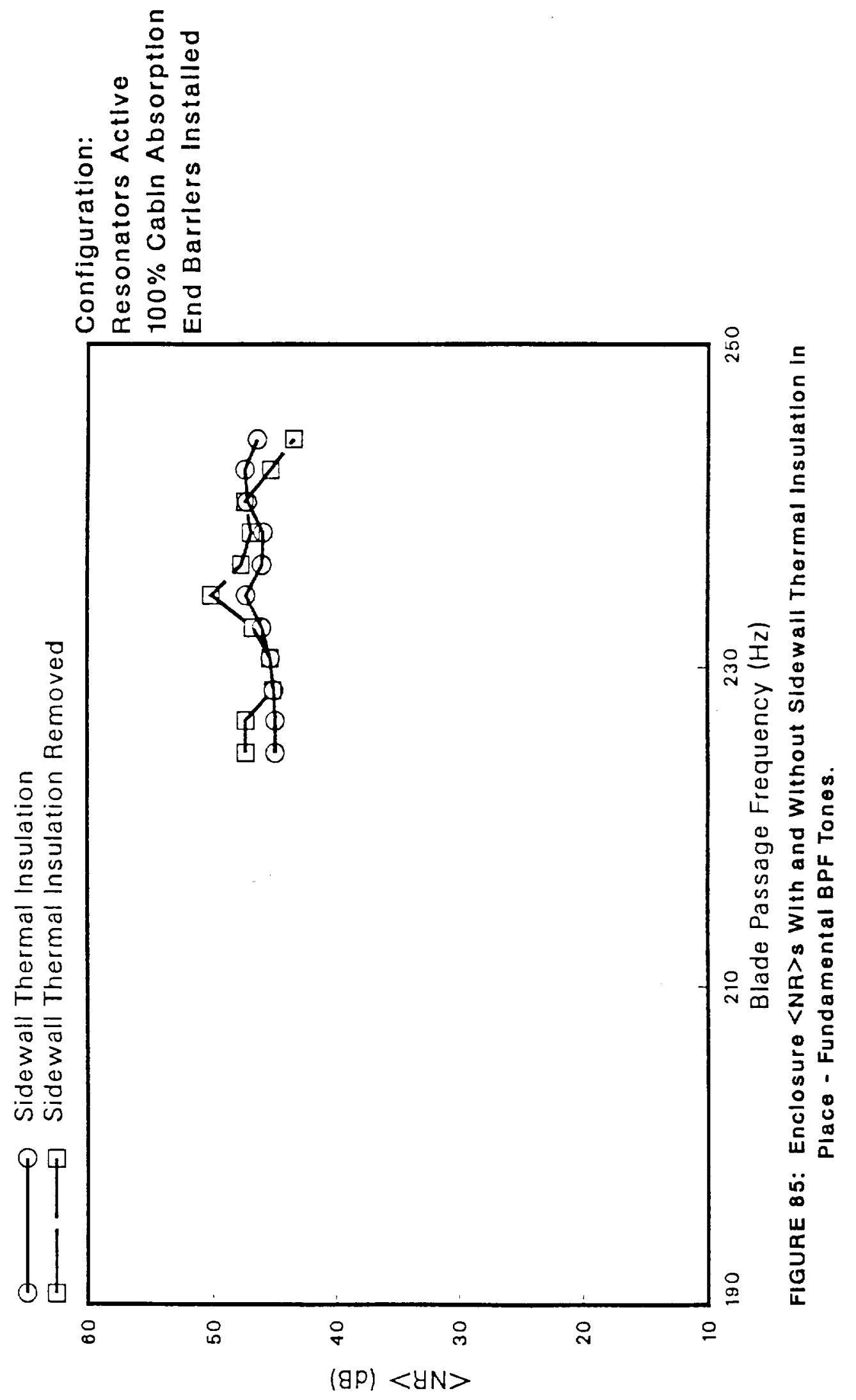




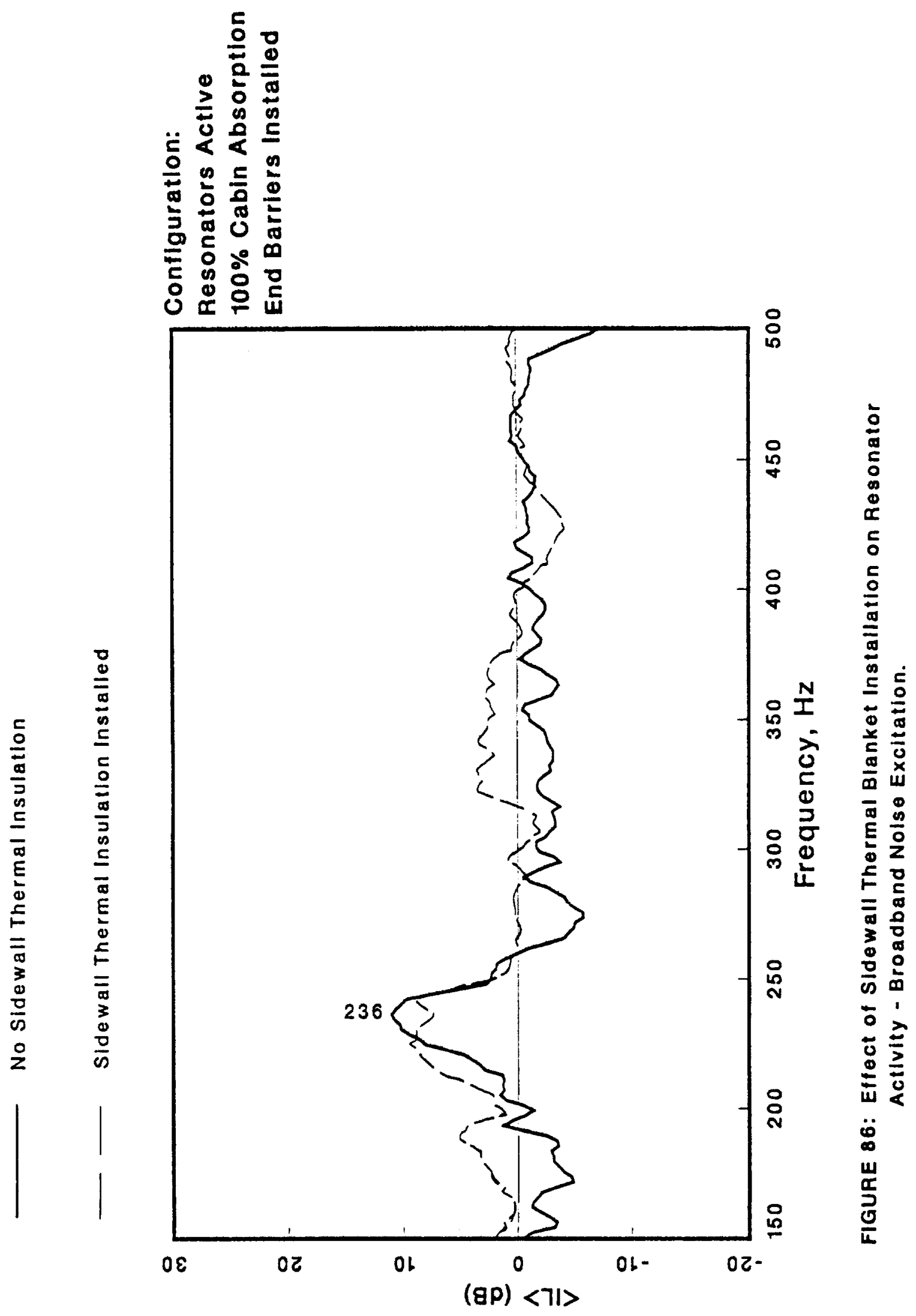




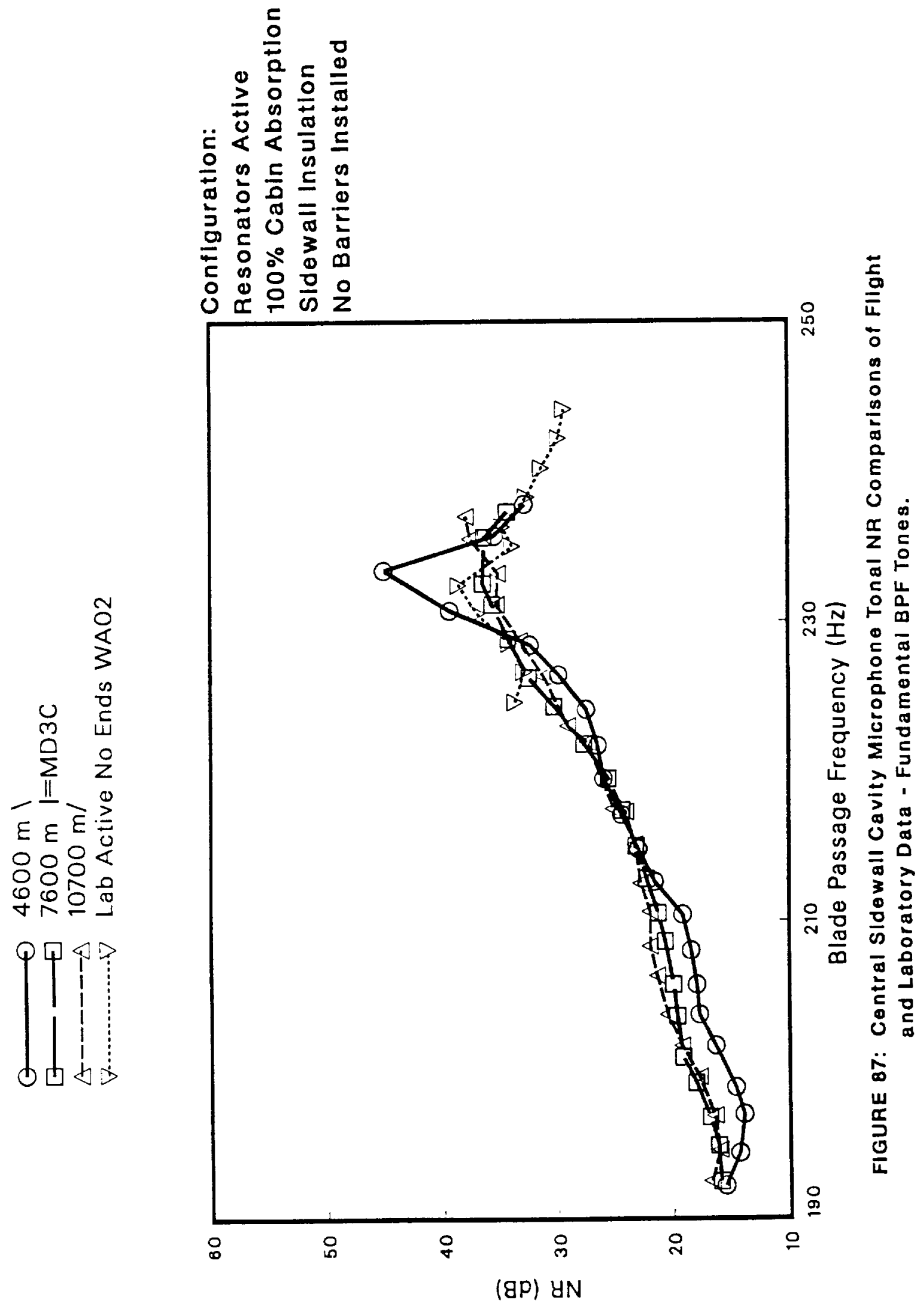




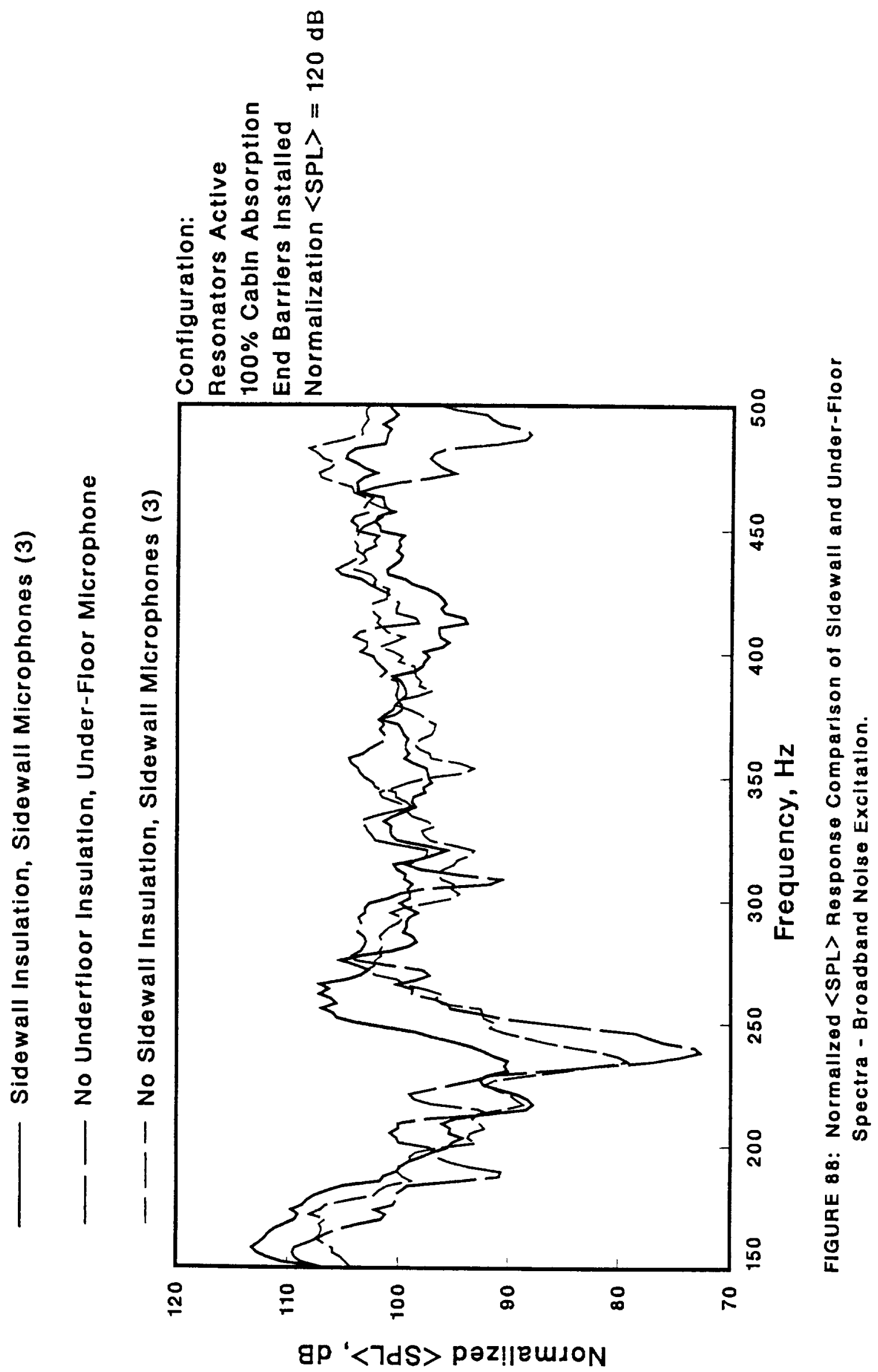




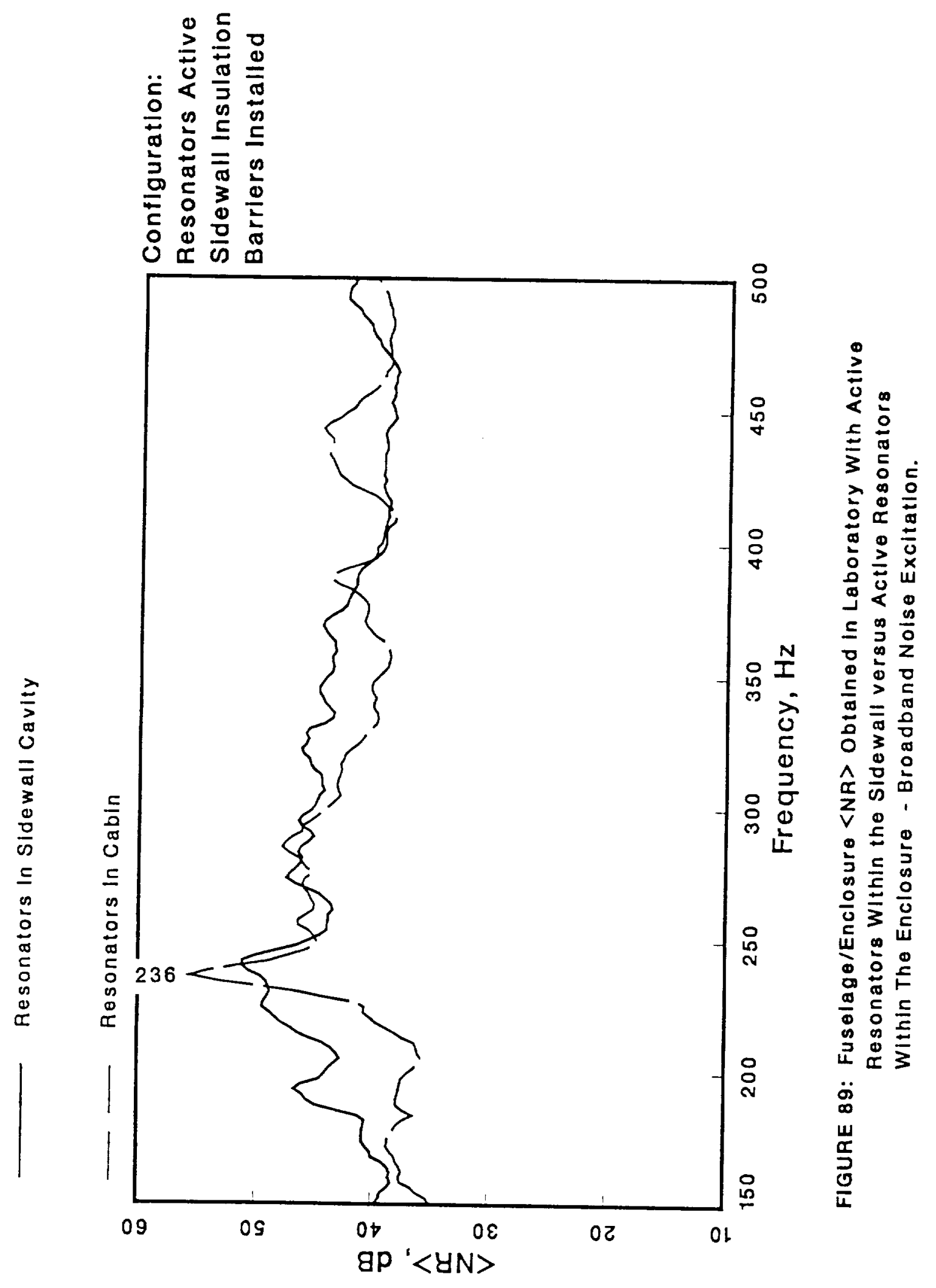





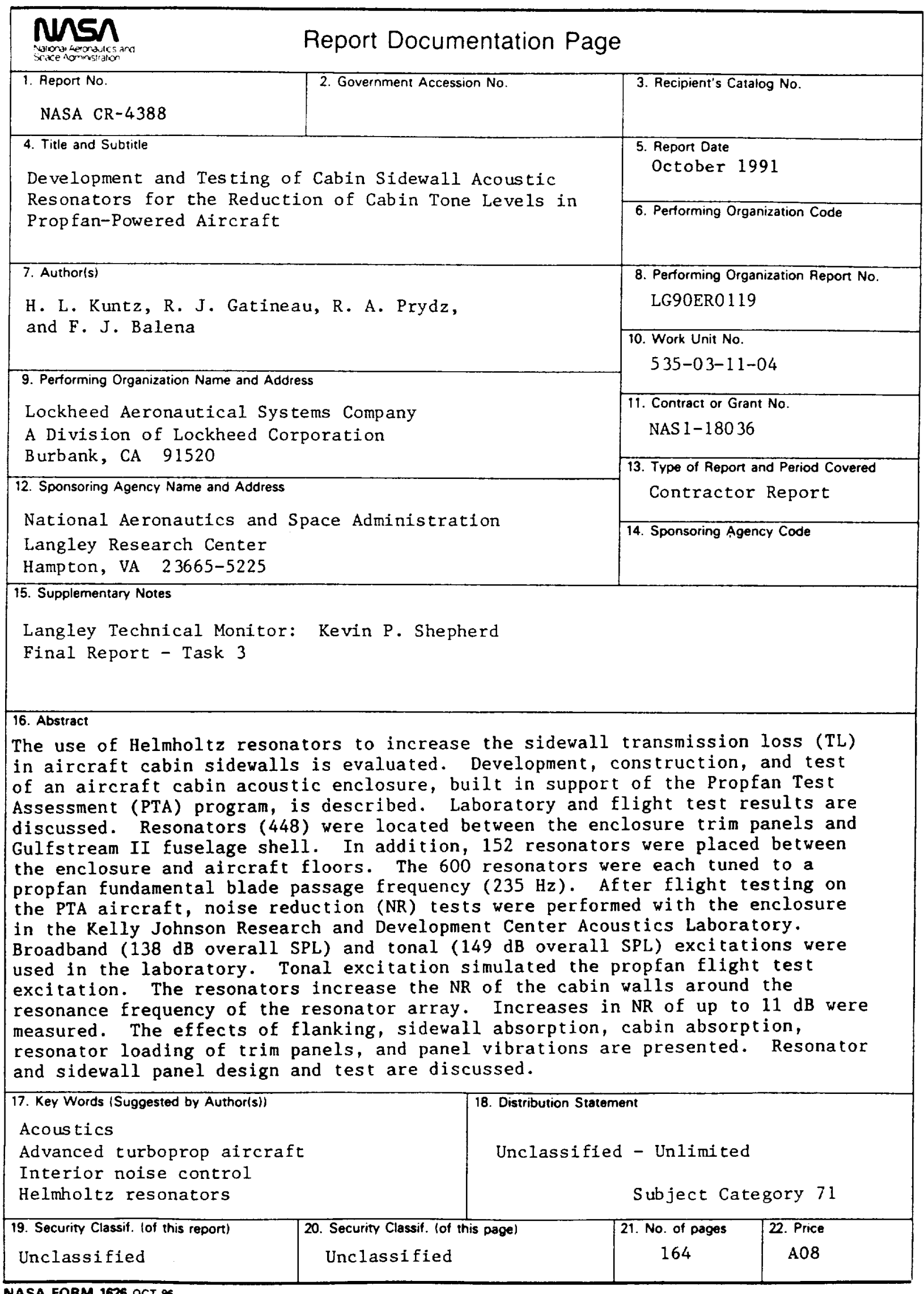

NASA FORM 1626 OCT $\$$ For sale by the National Technical Information Service, Springfield, Virginia 22161-2171 NASA-Langley, 1991 



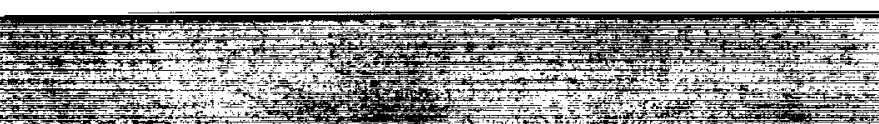
1.

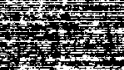 $\sqrt[3]{12}$ Hits?

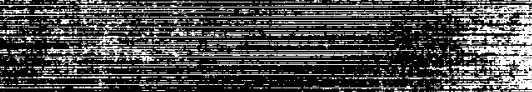

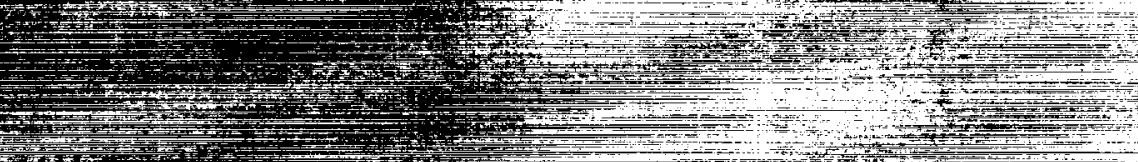

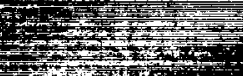
$\frac{3}{3+3}$

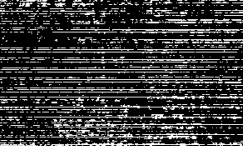
1.5.
8

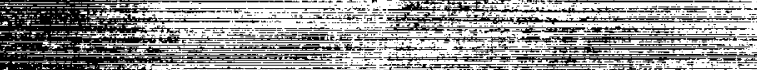

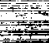

\section{tit}

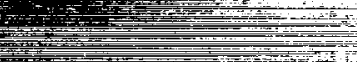

1.4.

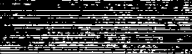

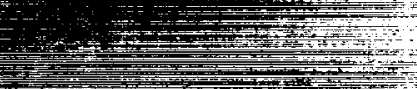

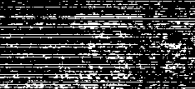

(1)

$403=1$

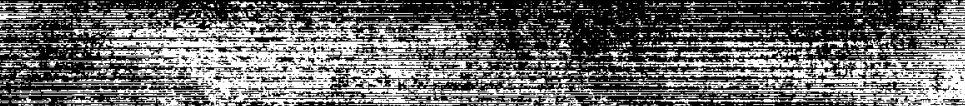

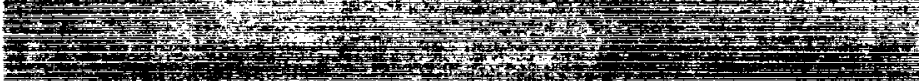

\section{(1)}

$\frac{1}{4+2}$

$$
12
$$

ind

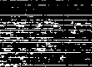

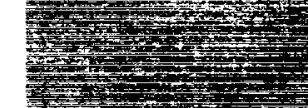

1.

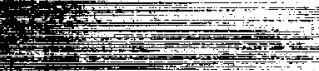

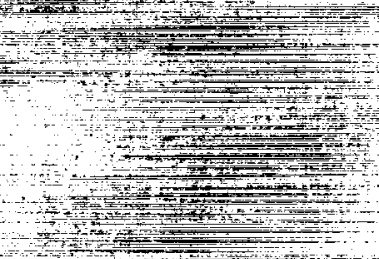

intint
T.t.

\section{5ing}

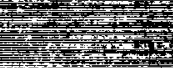

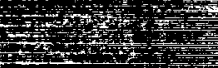
$-\frac{1}{2}$

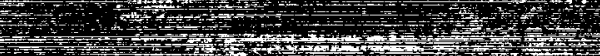




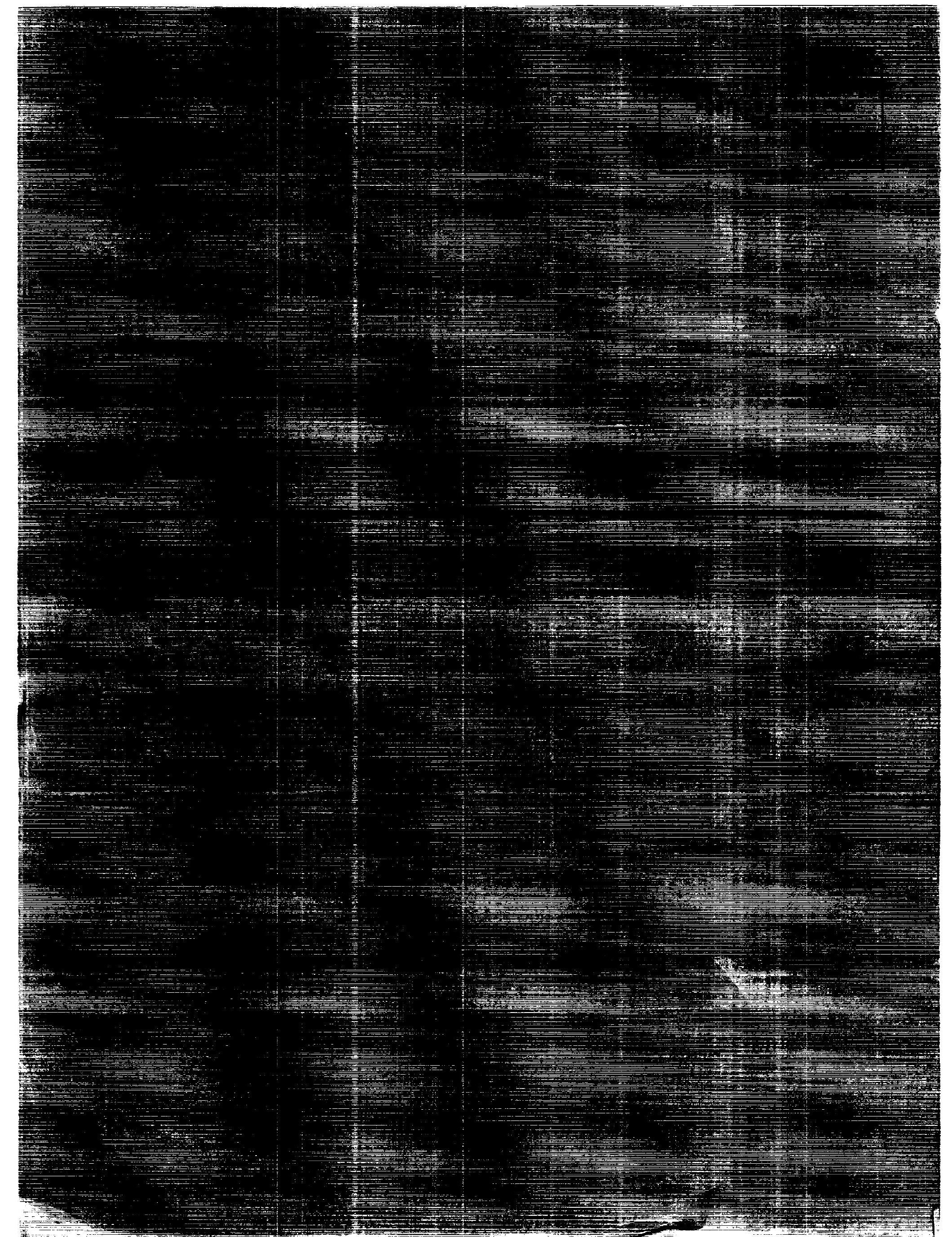

